\begin{abstract}
A41
Casa abierta al tiempo

UNIVERSIDAD AUTÓNOMA MEIROPOLITANA-IZTAPALAPA

DIVISIÓN DE CIENCIAS BÁSICAS E INGENIERIA

ÁREA DE QUÍMICA ANALÍTICA
\end{abstract}

\title{
ESTUDIO Y CARACTERIZACIÓN DE \\ LAS ESPECIES PODUCTO DE LA \\ HIDRÓLISIS DE B(III), Ga(III) e In(III) Y \\ SU INTERACCIÓN CON Al(III)
}

TESIS QUE PRESENTA EL

M.en Q. JULIO CÉSAR BOTELLO POZOS

PARA LA OBTENCIÓN DEL GRADO DE

DOCTOR EN CIENCIAS

ASESOR

Dra. MARÍA TERESA RAMÍREZ SILVA

MÉXICO, JULIO DEL 2003 


\title{
Al \\ Casa abierta al tiempo
}

UNIVERSIDAD AUTONOMA METROPOLITANA-IZTAPALAPA

DIVISIÓN DE CIENCIAS BÁSICAS E INGENIERIA

ÁREA DE QUIMICA ANALITICA

\author{
ESTUDIO Y CARACTERIZACIÓN DE \\ LAS ESPECIES PRODUCTO DE LA \\ HIDRÓLISIS DE B(III), Ga(III) e In(III) Y \\ SU INTERACCIÓN CON Al(III)
}

TESIS QUE PRESENTA EL

M. en Q. JULIO CÉSAR BOTELLO POZOS

PARA LA OBTENCIÓN DEL GRADO DE

DOCTOR EN CIENCIAS

Dra. MARÍA TERESA RAMÍREZ SILVA 


\section{AGRADECIMIENTOS.}

A CONACyT por la beca otorgada para la realización de estudios de Doctorado.

A PEMEX y al IMP que a través del proyecto FIES-96-31-III financiaron parcialmente e hicieron posible el desarrollo de este proyecto

Al Dr. José Manuel Domínguez por su ayuda y asesoría para la realización de este trabajo, así como por el animo que me indujo a proseguir en este proyecto

Al M. en C. Atilano Gutiérrez por su invaluable ayuda, sus valiosos comentarios y por todo el apoyo prestado para la realización e interpretación en los estudios de Resonancia Magnética Nuclear.

A la Dra. Maria Teresa Ramírez Silva y al Dr. Alberto Rojas Hernández por hacerme participe en este proyecto, por sus consejos y sus comentarios durante la realización y desarrollo de este trabajo.

Al Área de Química Analítica de la UAM Iztapalapa y a la Sección de Química Analítica de la FESCuautitlán (UNAM) por las facilidades prestadas para la realización de este trabajo.

Al Dr. A. Vacca y al Dr. P. Gans por proporcionarnos una copia del programa computacional SUPERQUAD.

Al Dr. I. Puigdomenech y al Profesor Josef Havel por la copia del programa MEDUSA

A los Drs. Marcos Solache R., Gretchen T. Lapidus L., Hugo Sánchez S. y Alma Revilla V. Por la revisión que hicieron y sus valiosos comentarios y observaciones a este trabajo.. 


\section{ÍNDICE}

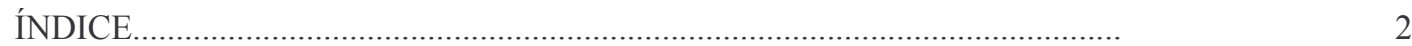

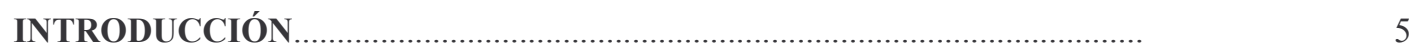

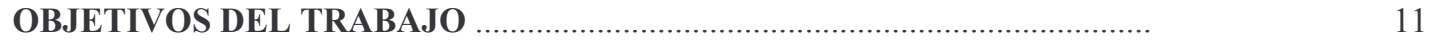

CAPÍTULO I. ESTUDIO Y CARACTERIZACIÓN DE LOS SISTEMAS BINARIOS: $\mathrm{Al}(\mathrm{III})-\mathrm{H}_{2} \mathrm{O}, \mathrm{Ga}(\mathrm{III})-\mathrm{H}_{2} \mathrm{O}, \mathrm{B}(\mathrm{III})-\mathrm{H}_{2} \mathrm{O}$ E In(III)- $\mathrm{H}_{2} \mathrm{O}$

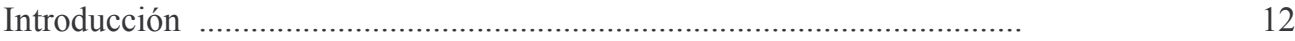

1.1. Sistema B(III)- $\mathrm{H}_{2} \mathrm{O}$

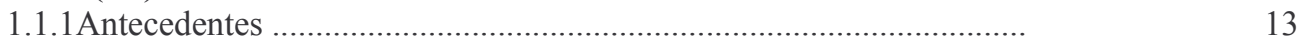

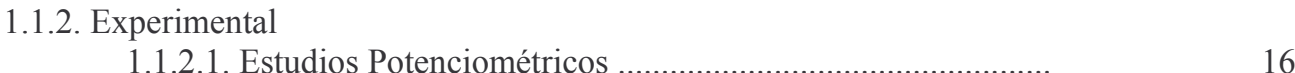

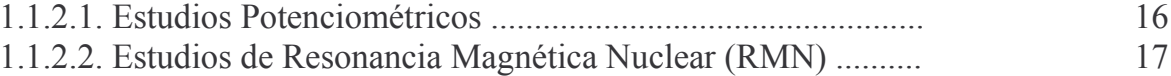

1.1.3. Resultados y Análisis

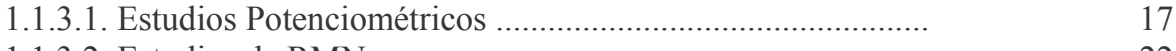

1.1.3.2. Estudios de RMN ......................................................... 22

1.1.4. Conclusiones ..................................................................................

1.2. Sistema $\mathrm{Al}(\mathrm{III})-\mathrm{H}_{2} \mathrm{O}$

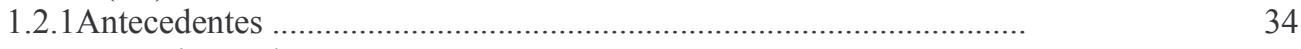

1.2.2. Experimental
1.2.2.1. Estudios Conductimétricos ....................................................

1.2.2.2. Estudios de Resonancia Magnética Nuclear (RMN) ............... 38

$\begin{array}{ll}\text { 1.2.3. Resultados y Análisis } & 38\end{array}$

1.2.3.1. Estudios Conductimétricos.................................................. 39

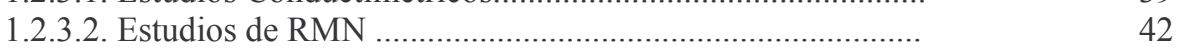

1.2.4. Conclusiones ................................................................................

\subsection{Sistema $\mathrm{Ga}(\mathrm{III})-\mathrm{H}_{2} \mathrm{O}$}

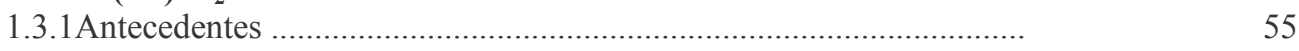

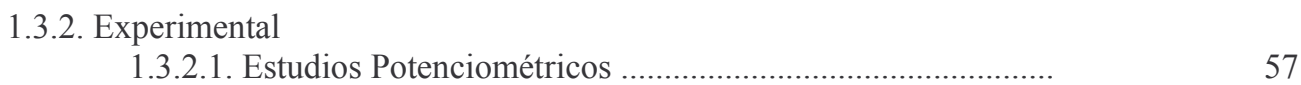

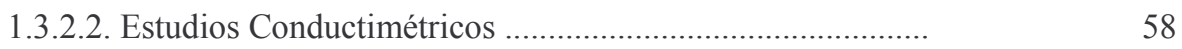

1.3.3. Resultados y Análisis

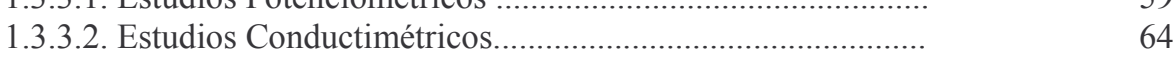

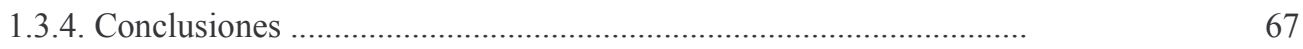

\subsection{Sistema In(III)- $\mathrm{H}_{2} \mathrm{O}$}

1.4.1Antecedentes .......................................................................................

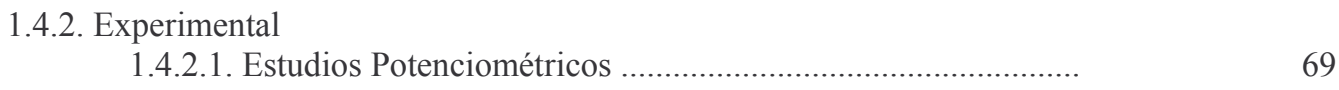

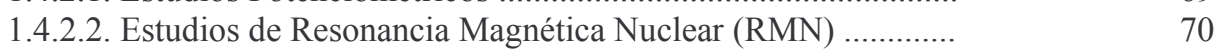

1.4.3. Resultados y Análisis

1.4.3.1. Estudios Potenciométricos ....................................................

1.4.3.2. Estudios de RMN ..............................................................

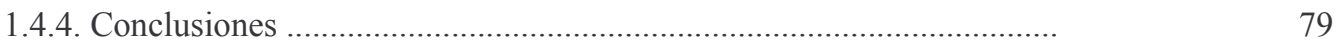

\section{CAPÍTULO II. ESTUDIO Y CARACTERIZACIÓN DE LOS SISTEMAS TERNARIOS:}


$\mathrm{Al}(\mathrm{III})-\mathrm{B}(\mathrm{III})-\mathrm{H}_{2} \mathrm{O}, \mathrm{Al}(\mathrm{III})-\mathrm{In}(\mathrm{III})-\mathrm{H}_{2} \mathrm{O}$ Y Al(III)-Ga(III)-- $\mathrm{H}_{2} \mathrm{O}$

Introducción

2.1. Sistema Al(III)-B(III)- $\mathrm{H}_{2} \mathrm{O}$

2.1.1. Experimental

2.1.1.1. Estudios Potenciométricos ..................................................

2.1.1.2. Estudios de RMN........................................................... 83

2.1.2. Resultados y Análisis

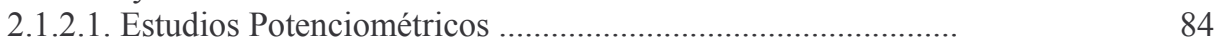

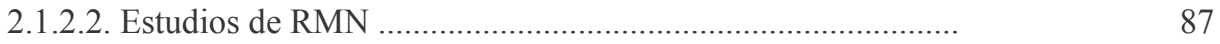

2.1.3. Conclusiones ............................................................................

2.2. Sistema $\mathrm{Al}(\mathrm{III})-\mathrm{In}(\mathrm{III})-\mathrm{H}_{2} \mathrm{O}$

2.2.1. Experimental

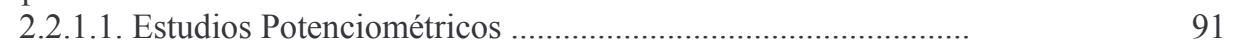

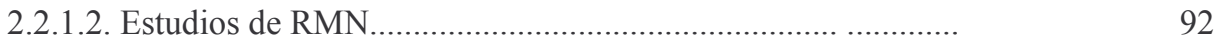

2.2.2. Resultados y Análisis

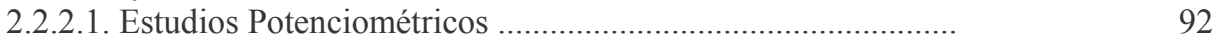

2.2.2.2. Estudios de RMN ............................................................ 96

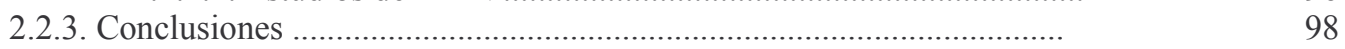

2.3. Sistema $\mathrm{Al}(\mathrm{III})-\mathrm{Ga}(\mathrm{III})-\mathrm{H}_{2} \mathrm{O}$

2.3.1. Experimental

2.3.1.1. Estudios Potenciométricos ............................................. 99

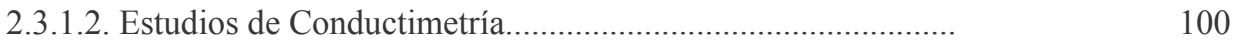

2.3.1.3. Estudios de RMN..........................................................

2.3.2. Resultados y Análisis

2.3.2.1. Estudios Potenciométricos ................................................ 102

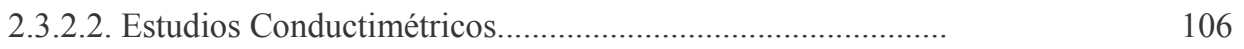

2.3.2.3. Estudios de RMN....................................................

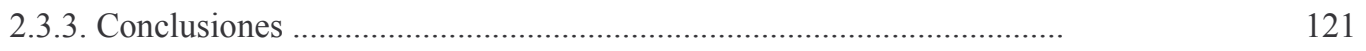

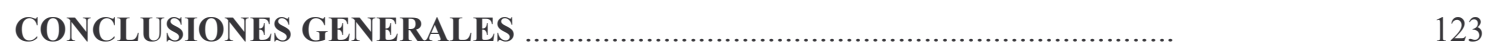

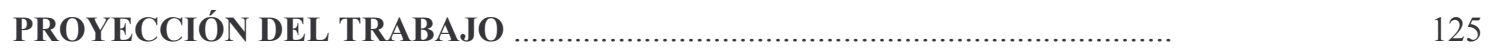

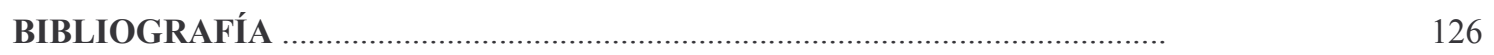

APÉNDICE I EQUILIBRIO DE HIDRÓLISIS Y MÉTODOS GRÁFICOS PARA SU REPRESENTACIÓN.

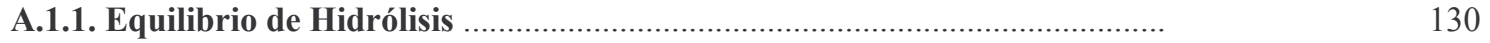

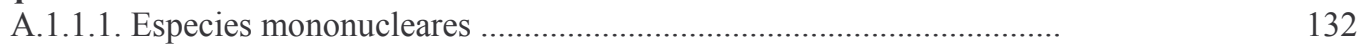

A.1.1.2. Especies polinucleares ....................................................... 133

A.1.2. Diagramas de Distribución.

A.1.2.1. Fracciones Molares de Componente 133

A.1.2.2. Fracciones Molares de Especie 133

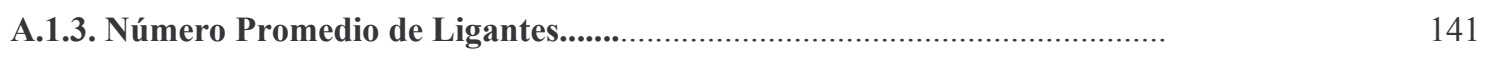

APÉNDICE II CONCEPTOS BÁSICOS DE RESONANCIA MAGNÉTICA NUCLEAR (RMN)

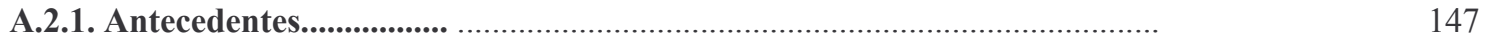

A.2.2. Propiedades Nucleares y la condición de resonancia ................................ 148 
A.2.3. Principales parámetros de RMN

A.2.3.1. Desplazamiento Químico y el Tensor de Protección

151

A.2.3.2. Acoplamiento Dipolar (D)

152

A.2.3.3. Acoplamiento Indirecto Espín-Espín (J)

153

A.2.3.4. Cuadrupolo Eléctrico

154

A.2.4. Determinación del Área para las Señales de RMN 


\section{INTRODUCCIÓN}

Los sistemas en equilibrio químico han sido ampliamente estudiados, ya que adquieren gran importancia en numerosos procesos. Un tipo particular de equilibrio químico de interés en diversos ámbitos de la ciencia y la tecnología es el proceso de hidrólisis, este se refiere a las reacciones químicas en las cuales una sustancia es “descompuesta” por el agua. La hidrólisis de especies inorgánicas se refiere a las reacciones que tienen lugar en soluciones de sales, en las cuales son formadas nuevas especies ionicas o precipitados como óxidos o hidróxidos. La hidrólisis de sales puede involucrar tanto al catión como al anión o bien a ambos, aunque generalmente los estudios se enfocan a la hidrólisis de cationes para formar especies solubles con hidróxido u oxi-complejos, así como la formación de precipitados de hidróxido ${ }^{1}$.

Prácticamente todos los cationes son capaces de formar hidroxo-complejos, lo cual no debe sorprendernos si consideramos que el ligando $\mathrm{OH}^{-}$esta siempre presente en el agua, en concentraciones que pueden variar en el intervalo de $10^{-1} \leq\left[\mathrm{OH}^{-}\right] \leq 10^{-14}$ esto como resultado de la disociación del agua, el valor de la concentración de los iones hidróxido dependerá por tanto del $\mathrm{pH}$ del sistema. 
La ecuación que describe la reacción de formación de productos solubles en el proceso de hidrólisis, así como la expresión de la constante de equilibrio respectiva (la cual generalmente se etiqueta con la letra griega $\beta$ ) se muestran en la ecuación I.1, debido a la diversidad de complejos que se pueden formar, el comportamiento del metal en una solución puede ser difícil de interpretar y esté será función tanto del $\mathrm{pH}$ como de la concentración, así como de la identidad y estabilidad de los productos formados en el proceso de hidrólisis².

$$
\begin{gathered}
\mathrm{xM}^{\mathrm{z+}}+\mathrm{yH}_{2} \mathrm{O} \leftrightarrow \mathrm{M}_{\mathrm{x}}(\mathrm{OH})_{\mathrm{y}}^{(\mathrm{xz}-\mathrm{y})}+\mathrm{yH}^{+} \\
\beta_{\mathrm{x}, \mathrm{y}}=\frac{\left[\mathrm{M}_{\mathrm{x}}(\mathrm{OH})_{\mathrm{y}}^{(\mathrm{xz}-\mathrm{y})}\right]\left[\mathrm{H}^{+}\right]^{\mathrm{y}}}{\left[\mathrm{M}^{\mathrm{z+}}\right]^{\mathrm{x}}}
\end{gathered}
$$

La determinación de las especies que se forman durante la hidrólisis, así como sus constantes de equilibrio generalmente esta acompañado de algunas dificultades como son:

(1) Los hidroxocomplejos formados pueden ser especies tanto mononucleraes como polinucleares, es decir especies que contienen más de un ion metálico. La diversidad de especies posibles y la aparición de estas en mayor o menor proporción de manera simultanea dificulta su caracterización y determinación de su estabilidad.

(2) El intervalo de pH en el cual se puede estudiar la formación de los hidroxocomplejos puede, en ocasiones, ser limitado por la precipitación del hidróxido del ion metálico. 
Sin embargo, pese a las dificultades antes señaladas se han desarrollado desde principios del siglo XX numerosos estudios para la identificación y caracterización de diversas especies formadas en la hidrólisis de la mayoría de los iones metálicos. El poder caracterizar a un sistema químico implica tanto la determinación de las posibles especies como su estabilidad, es decir, conocer la estequiometría de éstas y sus constantes de formación. Lo anterior hace que esto no sea un trabajo sencillo de llevar a cabo. Si bien es cierto, que en la actualidad es posible determinar las constantes de formación de las especies en solución con la ayuda de diversos métodos de análisis como son la potenciometría, espectrofotometría, coulombimetría, entre otras, y que el procesamiento de la información se puede realizar tanto por métodos gráficos como por métodos computacionales, siempre se requiere de un adecuado criterio para obtener al modelo más adecuado para el sistema en estudio, ya que para un mismo sistema se pueden obtener diversos modelos químicos. Esto nos lleva continuamente a la pregunta ¿Cuál será el modelo químico más adecuado?

La obtención de un modelo químico no debe limitarse solamente a la determinación de diferentes estequiometrías, sino a corroborar por medio de otras técnicas analíticas si el modelo propuesto por un programa computacional y/o metódos gráficos explica adecuadamente el comportamiento experimental.

En este trabajo se realiza el estudio de cuatro sistemas binarios y de tres sistemas ternarios con especies del grupo IIIA (aluminio, galio, indio y boro). La especiación de los sistemas se realiza por medio de técnicas potenciométricas, procesando la información con ayuda del programa SUPERQUAD, mientras que la validación de los modelos químicos 
propuestos se realiza con el desarrollo de métodos y/o técnicas como estudios del número promedio de ligandos, estudios de conductimetría y de resonancia magnética nuclear. La caracterización de estos sistemas está enfocada hacia la posible utilización de soluciones como medios de impregnación para arcillas que se emplean en la catálisis de hidrocarburos $^{3-6}$.

Así el propósito fundamental es el de poder determinar el mejor modelo químico para el sistema que permita conocer las condiciones de formación de las especies químicas que puedan ser empleadas como precursores para la impregnación de arcillas empleadas en materiales utilizados en catálisis. De esta forma se persigue tener una adecuada caracterización del sistema, de tal manera que los procesos de impregnación ya no se realicen empíricamente, sino que se puedan establecer previamente las condiciones convenientes para que se tenga a la especie catiónica adecuada que servirá como precursor del polioxocatión que se impregnará en las arcillas.

Además, paralelo a lo anterior, se desarrolla un algoritmo que permita relacionar las señales de resonancia magnética nuclear $(\mathrm{RMN})$ con las fracciones molares, ya que pese a que la resonancia magnética nuclear se ha desarrollado notablemente en los últimos años la explicación de las señales obtenidas solamente se asocia a la geometría de los núcleos y muy pocos estudios se han enfocado a tratar de dar una explicación cuantitativa de las señales $^{7,8}$. Por ello este trabajo propone una metodología nueva que permite asociar las áreas de las señales de RMN con las fracciones molares de especie. Con ello se hace posible el desarrollo de un estudio cuantitativo que no solo permita asociar por geometría las señales de RMN con especies químicas, y de esta manera hacer posible la 
determinación tanto de modelos químicos, y que también permita la corroboración y validación de constantes de formación asociadas a las posibles especies formadas en un sistema. De esta manera se sientan las bases para el posible desarrollo de métodos cuantitativos de análisis empleando esta técnica. 


\section{OBJETIVOS DEL TRABAJO}

Desarrollar un algoritmo que relacione las señales de Resonancia Magnética Nuclear con las fracciones molares, con la finalidad de generar una herramienta cuantitativa que ayude en la determinación de especies químicas en sistemas en solución.

Determinar las especies químicas y sus constantes de formación de los sistemas binarios de $\mathrm{Al}(\mathrm{III})-\mathrm{H}_{2} \mathrm{O}, \quad \mathrm{Ga}(\mathrm{III})-\mathrm{H}_{2} \mathrm{O}, \quad \mathrm{B}(\mathrm{III})-\mathrm{H}_{2} \mathrm{O}$ e $\mathrm{In}(\mathrm{III})-\mathrm{H}_{2} \mathrm{O}$ mediante el procesamiento de información potenciométrica con el programa SUPERQUAD y validar los modelos químicos con estudios de número promedio de ligandos, de conductimetría y de resonancia magnética multinuclear.

Determinar las posibles especies químicas que se pudiesen formar, y sus constantes respectivas, en los sistemas ternarios de $\mathrm{Al}(\mathrm{III})-\mathrm{Ga}(\mathrm{III})-\mathrm{H}_{2} \mathrm{O}, \mathrm{Al}(\mathrm{III})-\mathrm{B}(\mathrm{III})-\mathrm{H}_{2} \mathrm{O}$, y $\mathrm{Al}(\mathrm{III})-$ In(III)- $\mathrm{H}_{2} \mathrm{O}$ mediante el procesamiento de información potenciométrica con el programa computacional SUPERQUAD y realizar la validación de los modelos químicos. 


\section{CAPÍTULO I}

\section{ESTUDIO Y CARACTERIZACIÓN DE LOS SISTEMAS BINARIOS: $\mathrm{Al}(\mathrm{III})-\mathrm{H}_{2} \mathrm{O}, \mathrm{Ga}(\mathrm{III})-\mathrm{H}_{2} \mathrm{O}, \mathrm{B}(\mathrm{III})-\mathrm{H}_{2} \mathrm{O}$ E In(III)- $\mathrm{H}_{2} \mathrm{O}$}

\section{INTRODUCCIÓN}

La problemática de contar con materiales catalíticos mejores, más eficientes y más rentables para la industria petrolera ha generado que en los últimos años se hayan venido desarrollando diversas clases de materiales sólidos con características micro y mesoporosas mediante la intercalación, dentro de las capas de materiales inorgánicos, de especies iónicas polinucleares, tales como silicatos, fosfatos de circonio y diversos óxidos e hidróxidos metálicos ${ }^{9-13}$. La sustitución de especies de algunos cationes metálicos trivalentes, tales como $\mathrm{Al}(\mathrm{III}), \mathrm{B}(\mathrm{III}), \mathrm{Fe}(\mathrm{III})$ entre otros, generalmente llevan a la formación de sitios ácidos de Brønsted, cuya fuerza y densidad dependerá de la naturaleza del catión. Materiales de este tipo tienen aplicaciones en catálisis ácida como la desarrollada para alquilación aromática, el cracking de petróleo y en el mejoramiento de petróleo pesado ${ }^{14}$. De aquí que se ha despertado el interés por desarrollar la síntesis de nuevos tipos de materiales que permitan realizar este tipo de intercalación para formar productos pilareados con alta estabilidad térmica. 
El conocimiento de las posibles especies que se forman en solución, así como de sus constantes de formación, es esencial para poder determinar condiciones tanto de concentración de catión, así como de pH que permitan tener a los precursores químicos de las especies de interés para el proceso de intercalación en las arcillas. Por ello, esta parte del trabajo esta enfocada al estudio de sistemas binarios que comprenden a cationes del grupo IIIA ( $\mathrm{Al}(\mathrm{III})$, $\mathrm{Ga}(\mathrm{III}), \mathrm{B}(\mathrm{IIII}), \operatorname{In}(\mathrm{III}))$, analizando la hidrólisis de estos en agua para caracterizar las especies que se forman y efectuar el cálculo de sus constantes, para poder de esta manera determinar los posibles precursores para el pilareado de materiales sólidos con fines catalíticos.

\subsection{SISTEMA B(III)- $\mathrm{H}_{2} \mathrm{O}$}

\subsubsection{ANTECEDENTES}

La hidrólisis de boro(III) en solución acuosa, si bien ha sido estudiada por algunos investigadores, en su mayoría estos estudios se han enfocado a la denominada reacción principal de hidrólisis en la que se forma el ion borato a partir del ácido bórico. El valor de la constante de formación para el ion borato aún no ha sido totalmente definida ya que existe una gran variación en los valores hasta ahora determinados ${ }^{1,16-23}$. Aunque se ha planteado la posible formación de especies polinucleares (poliboratos), son pocos los autores que han podido determinar a estas especies y a sus constantes de formación. Baes y 
Mesmer $^{1}$ proponen que es factible la formación de poliboratos durante la hidrólisis, sin embargo hacen mención de la dificultad que representa el poder determinarlas ya que estas especies dependerán marcadamente de la concentración de boro (III). Establecen estos autores que los poliboratos más importantes que se forman son iones mononegativos.

Los estudios para la determinación de las especies de boro, producto de la hidrólisis, se han realizado en su mayoría por medio de potenciometría. En años recientes Flanagan y colaboradores ${ }^{15}$ mediante resonancia magnética nuclear, pusieron en evidencia la formación de un dímero en estado sólido y en solución acuosa aunque no determinaron la constante de formación de esta especie. Lo anterior no ha permitido que hasta el momento se cuente con un modelo químico adecuado para las especies formadas en el proceso de hidrólisis, existiendo divergencia sobre los posibles poliboratos que se pudiesen formar así como de sus constantes.

Tabla 1.1. Constantes de formación propuestas para la hidrólisis de B(III)

\begin{tabular}{|l|c|c|c|}
\hline \multicolumn{1}{|c|}{ AUTOR(ES) } & $\begin{array}{l}\text { Especies } \\
\text { Químicas }\end{array}$ & $-\log \boldsymbol{\beta}^{*}$ & Método \\
\hline Owen y King $^{16}$ & $\mathrm{~B}(\mathrm{OH})_{4}{ }^{-}$ & 9.237 & Potenciometría \\
\hline Ingri N. $^{17}$ & $\mathrm{~B}(\mathrm{OH})_{4}{ }^{-}$ & $8.74(0.08)$ & \\
& $\mathrm{B}_{3} \mathrm{O}_{3}(\mathrm{OH})_{4}{ }^{-}$ & $6.58(0.06)$ & Potenciometría \\
& $\mathrm{B}_{4} \mathrm{O}_{5}(\mathrm{OH})_{4}{ }^{-2}$ & $14.41(0.12)$ & \\
& $\mathrm{B}_{3} \mathrm{O}_{3}(\mathrm{OH})_{5}{ }^{-2}$ & $16.14(0.16)$ & \\
\hline Baes C.F. y Mesmer R.E. & $\mathrm{B}(\mathrm{OH})_{4}{ }^{-}$ & 8.94 & \\
& $\mathrm{~B}_{2} \mathrm{O}(\mathrm{OH})_{5}{ }^{-}$ & 8.27 & Potenciometría \\
& $\mathrm{B}_{3} \mathrm{O}_{3}(\mathrm{OH})_{4}{ }^{-}$ & 6.90 & \\
\hline Gold V. y Lowe B. $^{18}$ & $\mathrm{~B}_{4} \mathrm{O}_{5}(\mathrm{OH})_{4}{ }^{2-}$ & 15.15 & \\
\hline
\end{tabular}




\begin{tabular}{|l|c|c|c|}
\hline Khoo K. $^{19}$ & $\mathrm{~B}(\mathrm{OH})_{4}^{-}$ & 7.308 & Potenciometría \\
\hline Feakins D. y Khoo K. ${ }^{20}$ & $\mathrm{~B}(\mathrm{OH})_{4}^{-}$ & 9.30 & Potenciometría \\
\hline
\end{tabular}

Tabla 1.1. Constantes de formación propuestas para la hidrólisis de B(III) (Continuación)

\begin{tabular}{|l|l|c|c|}
\hline \multicolumn{1}{|c|}{ AUTOR(ES) } & $\begin{array}{l}\text { Especies } \\
\text { Químicas }\end{array}$ & $-\boldsymbol{- l o g} \boldsymbol{\beta}^{*}$ & Método \\
\hline \multirow{2}{*}{ Spessard J. ${ }^{21}$} & $\mathrm{~B}(\mathrm{OH})_{4}^{-}$ & 8.56 & \\
& $\mathrm{~B}_{3} \mathrm{O}_{3}(\mathrm{OH})_{4}^{-}$ & 16.06 & Potenciometría \\
& $\mathrm{B}_{4} \mathrm{O}_{5}(\mathrm{OH})_{4}^{-2}$ & 14.23 & \\
& $\mathrm{~B}_{3} \mathrm{O}_{3}(\mathrm{OH})_{5}^{-2}$ & 6.56 & \\
\hline Crisponi G, Nurchi V., Ganadu M y $^{-2}$ & $\mathrm{~B}(\mathrm{OH})_{4}^{-}$ & 9.07 & Potenciometría \\
Lubinu G. $^{22}$ & & & \\
\hline Rogers H. Y Van der Berg C. & & & \\
& $\mathrm{B}(\mathrm{OH})_{4}^{-}$ & 8.85 & Potenciometría \\
\hline
\end{tabular}

En la información reportada en la Tabla 1.1 es posible apreciar que diferentes autores que han estudiado el sistema $\mathrm{B}(\mathrm{III})-\mathrm{H}_{2} \mathrm{O}$, reportan diferencias en los modelos químicos obtenidos. Dichas diferencias se hacen más considerables cuando uno analiza las diversas propuestas sobre las posibles especies polinucleares, no sólo en cuanto a las especies, sino a los valores de las constantes respectivas. Lo anterior dificulta la caracterización química de sistemas en donde el B(III) se hidroliza, por ello en este trabajo se busca determinar a las posibles especies que se forman durante la hidrólisis de B(IIII), realizando esto no sólo por estudios potenciométricos, sino también con apoyo de la técnica de resonancia magnética nuclear. Lo anterior para proporcionar información acerca de la 
estructura de las especies que se forman y con ello dar una mayor validez al modelo planteado.

\subsubsection{EXPERIMENTAL}

\subsubsection{Estudios Potenciométricos}

Se prepararon soluciones acuosas de $\mathrm{B}(\mathrm{III})$ a partir de $\mathrm{H}_{3} \mathrm{BO}_{3}$ (Merck 99.8\%) en un intervalo de $1.05 \times 10^{-2} \mathrm{M}$ a $63.50 \times 10^{-2} \mathrm{M}$, en la preparación de todas las soluciones se empleó agua desionizada y descarbonatada de Tipo I (desionizador Millipore). La concentración analítica de $\mathrm{B}(\mathrm{III})$ se determinó por medio de la valoración.

Se realizaron valoraciones de al menos dos muestras de cada solución con soluciones estandarizadas de hidróxido de sodio, las cuales fueron preparadas a partir de una solución acuosa al 50\% en masa de hidróxido de sodio tomando alícuotas del sobrenadante de esta, empleando agua desionizada y hervida, y sometiendo a un burbujeo de nitrógeno para la eliminación de los carbonatos. Las variaciones de $\mathrm{pH}$ y de potencial durante la valoración se midieron empleando un pH-metro Radiometer Tacussel LPH430T $(\Delta \mathrm{pH}=0.001)$, utilizando un electrodo de vidrio combinado con referencia interna de $\mathrm{Ag} / \mathrm{AgCl}_{(\mathrm{s})}$ y efectuando la calibración del equipo con sistemas buffer de referencia de

$\mathrm{pH}=4.005$ y 7.001. En todos los casos se realizaron al menos 60 mediciones. Todas las valoraciones se efectuaron manteniendo la temperatura constante a $25.0^{\circ} \mathrm{C}\left(\Delta \mathrm{T}=0.1^{\circ} \mathrm{C}\right)$ 
por medio de un baño (VWR, modelo 12101-10) y empleando celdas de vidrio de doble camisa. Durante la experimentación se mantuvo un flujo constante de nitrógeno de alta pureza con la finalidad de evitar la carbonatación del sistema

\subsubsection{2. . Estudios de Resonancia Magnética Nuclear (RMN)}

Se prepararon soluciones acuosas de $\mathrm{B}(\mathrm{III})$ de concentración $0.075 \mathrm{M}, 0.500 \mathrm{M}$ y 0.700M a partir de $\mathrm{H}_{3} \mathrm{BO}_{3}$ (Merck 99.8\%). Se realizó la hidrólisis de boro desde una relación $\mathrm{OH} / M$ de 0.00 hasta aproximadamente 1.0 , con soluciones estándar de hidróxido de sodio, en todos los casos se registró el pH de la solución obtenida a $25.0^{\circ} \mathrm{C}$.

Los espectros de Resonancia Magnética Nuclear (RMN) de ${ }^{11} \mathrm{~B}$ se obtuvieron con un espectrometro Bruker DMX-500 a una temperatura de $25^{\circ} \mathrm{C}$, empleando $\mathrm{BF}_{3}-\mathrm{O}_{(}\left(\mathrm{C}_{2} \mathrm{H}_{5}\right)_{2}$ como referencia de desplazamiento químico, la frecuencia de excitación usada para el núcleo de boro fue igual a $160.46 \mathrm{MHz}$.

\subsubsection{RESULTADOS Y ANÁLISIS}

\subsubsection{Estudios de Potenciométricos}

Las curvas de valoración para las soluciones acuosas de ácido bórico se muestran en la Figura 1.1. En ellas se puede observar un comportamiento sigmoidal, característico de las valoraciones ácido-base, en las cuales se observa un incremento del $\mathrm{pH}$ a lo largo de 
la valoración. En aproximadamente $\mathrm{OH} / \mathrm{B}=1.0$ hay una variación significativa de este parámetro. Los datos de $\mathrm{pH}$ obtenidos para las valoraciones potenciométricas fueron alimentados al programa SUPERQUAD ${ }^{24,25}$, con la finalidad de refinar las constantes de formación para las especies que se forman en el proceso de hidrólisis de B(III). Para ello se consideró tanto a especies monoméricas como polinucleares de acuerdo al equilibrio reportado por Baes y Mesmer ${ }^{1}$, representado por la ecuación 1.1.

$$
\begin{aligned}
& \mathrm{iB}(\mathrm{OH})_{3} \leftrightarrow \mathrm{B}_{\mathrm{i}} \mathrm{O}_{\mathrm{m}}(\mathrm{OH})_{3 \mathrm{i}+\mathrm{j}-2 \mathrm{~m}}^{\mathrm{j}-}+\mathrm{jH} \mathrm{H}^{+}+(\mathrm{m}-\mathrm{j}) \mathrm{H}_{2} \mathrm{O} \\
& * \beta_{\mathrm{ij}}=\frac{\left[\mathrm{B}_{\mathrm{i}} \mathrm{O}_{\mathrm{m}}(\mathrm{OH})_{3 i+j-2 \mathrm{~m}}^{\mathrm{j}-}\right]\left[\mathrm{H}^{+}\right]^{\mathrm{j}}}{\left[\mathrm{B}(\mathrm{OH})_{3}\right]^{\mathrm{i}}}
\end{aligned}
$$

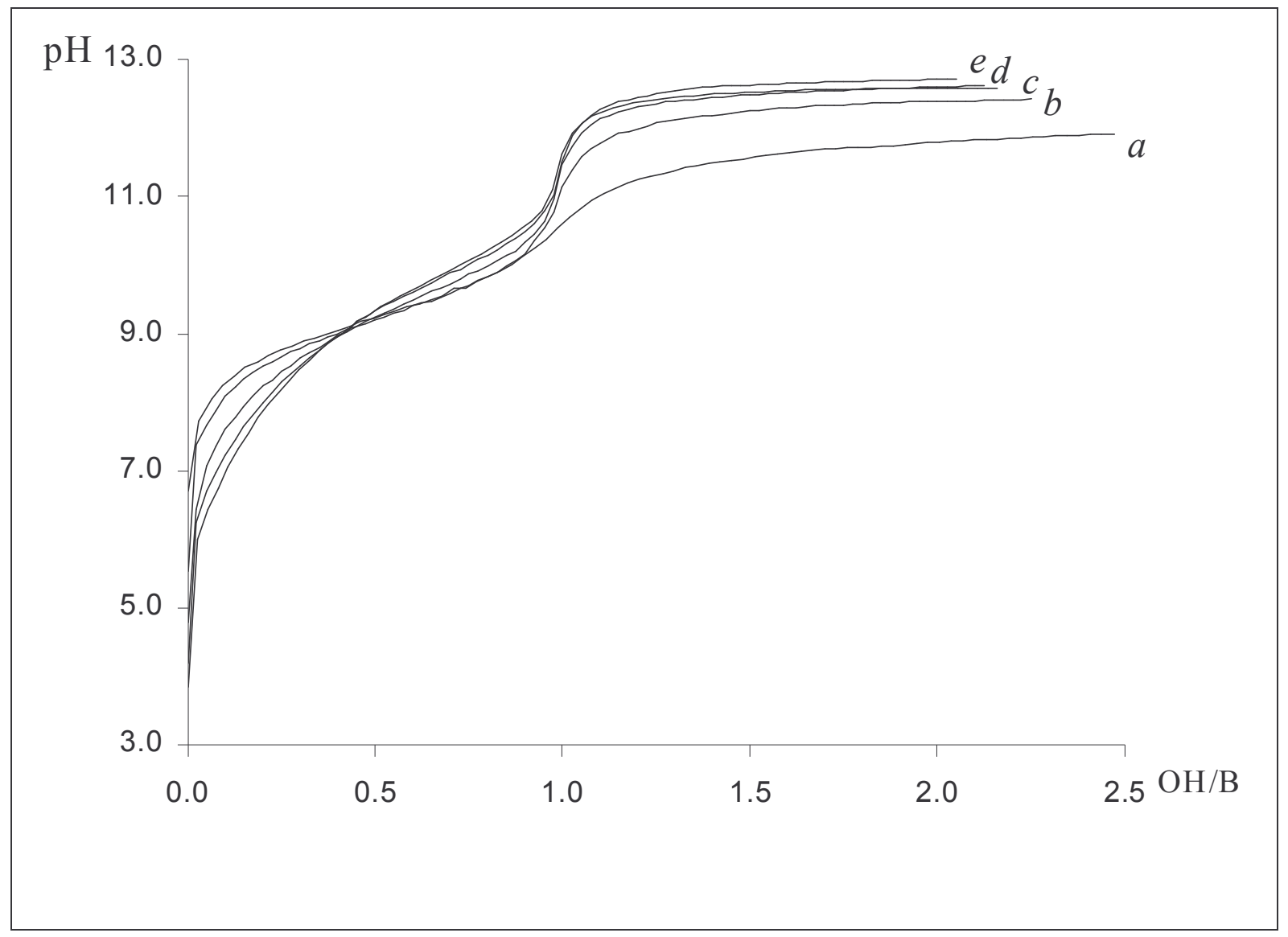


Figura 1.1. Curvas típicas de valoración de $\mathrm{H}_{3} \mathrm{BO}_{3}$ con $\mathrm{NaOH}$ estándarizado (a) $0.010 \mathrm{M}$, (b) 0.100 M, (c) $0.290 \mathrm{M}$, (d) $0.500 \mathrm{M}$, (e) $0.640 \mathrm{M}$.

El mejor refinamiento obtenido para este sistema se muestra en la Tabla 1.2, en la cual se puede apreciar la formación de una especie monomérica, así como la formación de tres especies polinucleares., las cuales como establecen Baes y Mesmer $^{1}$ son iones mononegativos . La Figura 1.2 muestra las posibles estructuras de las especies formadas en la hidrólisis de boro(III) a partir de ácido bórico.

Tabla 1.2 Valores de las constantes de formación para el mejor refinamiento obtenido a partir de datos potenciométricos procesados con SUPERQUAD para el sistema B(III)$\mathrm{H}_{2} \mathrm{O}^{\mathrm{a}}$.

\begin{tabular}{ccc}
\hline Especies & $-\log * \beta$ & Parámetros \\
& & Estadísticos \\
\hline $\mathrm{B}(\mathrm{OH})_{4}$ & $9.169 \pm 0.011$ & $\sigma^{\mathrm{c}}=3.076$ \\
$\mathrm{~B}_{2} \mathrm{O}(\mathrm{OH})_{5}^{-}$ & $8.553 \pm 0.077$ & $\mathrm{U}^{\mathrm{d}}=531.9$ \\
$\mathrm{~B}_{3} \mathrm{O}_{3}(\mathrm{OH})_{4}^{-}$ & $7.607 \pm 0.089$ & $\chi^{2 \mathrm{e}}=98.62$ \\
$\mathrm{~B}_{5} \mathrm{O}_{6}(\mathrm{OH})_{4}^{-}$ & $7.345^{\mathrm{b}}$ &
\end{tabular}

${ }^{a}$ Refinamiento realizado con 600 datos de las valoraciones potenciométricas. ${ }^{\mathrm{b}}$ valor mantenido constante durante el refinamiento ${ }^{\mathrm{c}}$ Desviación estándar total para el refinamiento ${ }^{\mathrm{d}}$ Suma de los residuales al cuadrados del potencial de celda. ${ }^{\mathrm{e}} \mathrm{Chi}$-cuadrada para el refinamiento

Por otra parte, con base en la información experimental obtenida potenciométricamente se construyeron las curvas experimentales de la función de formación de carga por átomo de boro $\mathrm{Z}_{\mathrm{q}}$, (como lo sugiere Baes y Mesmer ${ }^{1}$ para este sistema), para lo cual se empleó la ecuación 1.2. En la Figura 1.3 se muestran las curvas 
obtenidas, observándose que existe un desplazamiento de la función en relación al pH lo cual es característico para sistemas en los que se forman especies polinucleares. Además es importante destacar el hecho de que todas las curvas alcancen un valor máximo de uno lo cual sugiere, como ya se había mencionado, la formación de complejos monocargados.

$$
\mathrm{Z}_{\mathrm{q}}=-\left[\frac{\left[\mathrm{Na}^{+}\right]+\left[10^{-\mathrm{pH}}\right]-\left[\mathrm{K}_{\mathrm{w}} / 10^{-\mathrm{pH}}\right]-\left[\mathrm{Cl}^{-}\right]}{[\mathrm{B}(\mathrm{III})]_{\text {total }}}\right]
$$

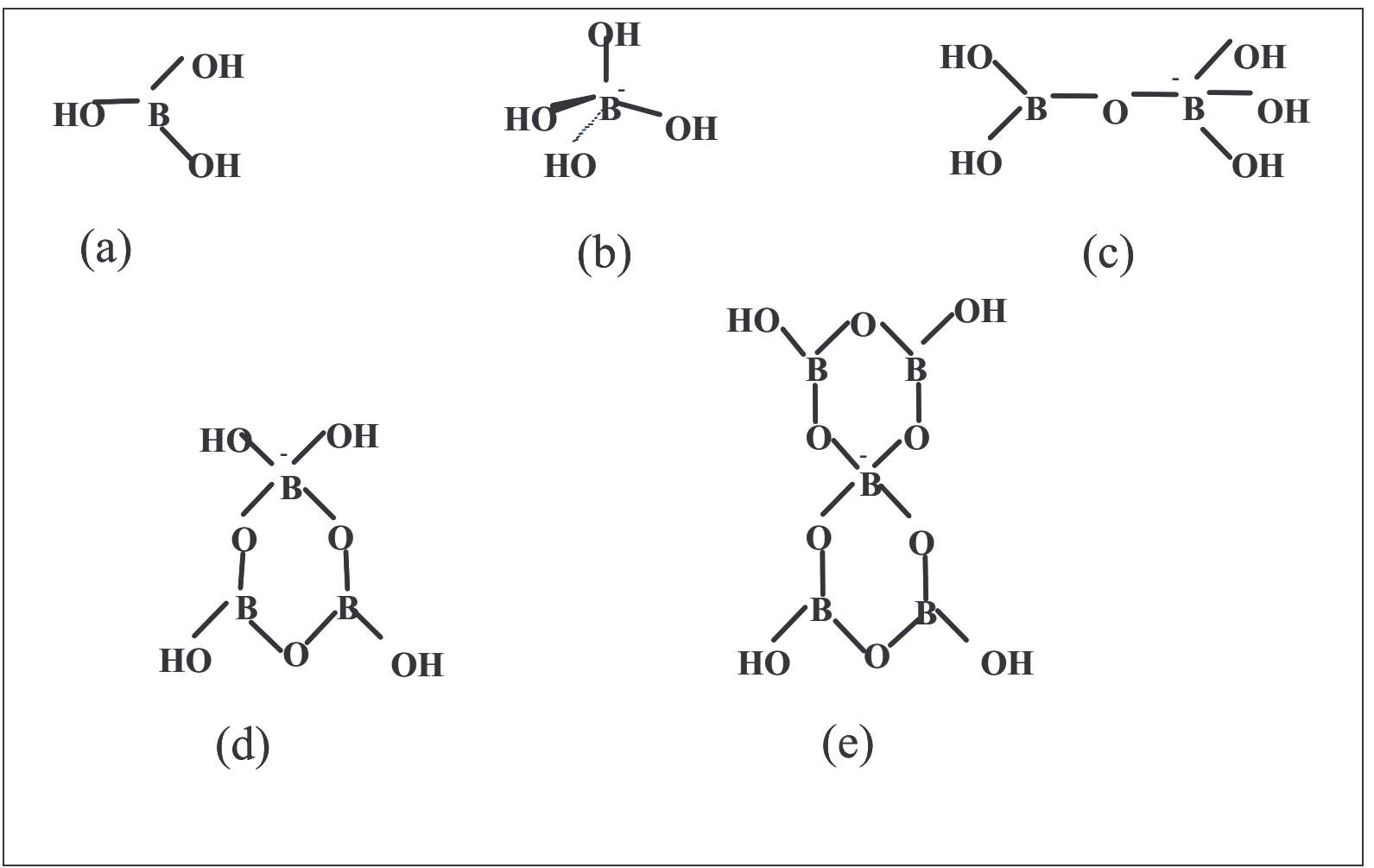

Figura 1.2. Posibles estructuras de las especies que se forman en la hidrólisis de B(III) en solución acuosa de acuerdo al modelo obtenido por el procesamiento de información potenciométrica con SUPERQUAD

Las funciones de formación de carga por átomo de boro se ajustaron gráficamente mediante la comparación de aquellas determinadas con base en la información 
experimental y las calculadas a partir de la ecuación 1.3. El mejor ajuste obtenido para los resultados experimentales se muestra en la Figura 1.4, en la que se observa que el modelo químico propuesto en la Tabla 1.2 explica satisfactoriamente el comportamiento experimental, ya que el ajuste obtenido es bastante concordante con los refinamientos realizados con SUPERQUAD.

$$
Z_{q}=-\left[\sum_{i}\left(\sum_{j}\left\{\left(\frac{3 i-j}{i}\right) F_{i j}\right\}\right)\right]
$$

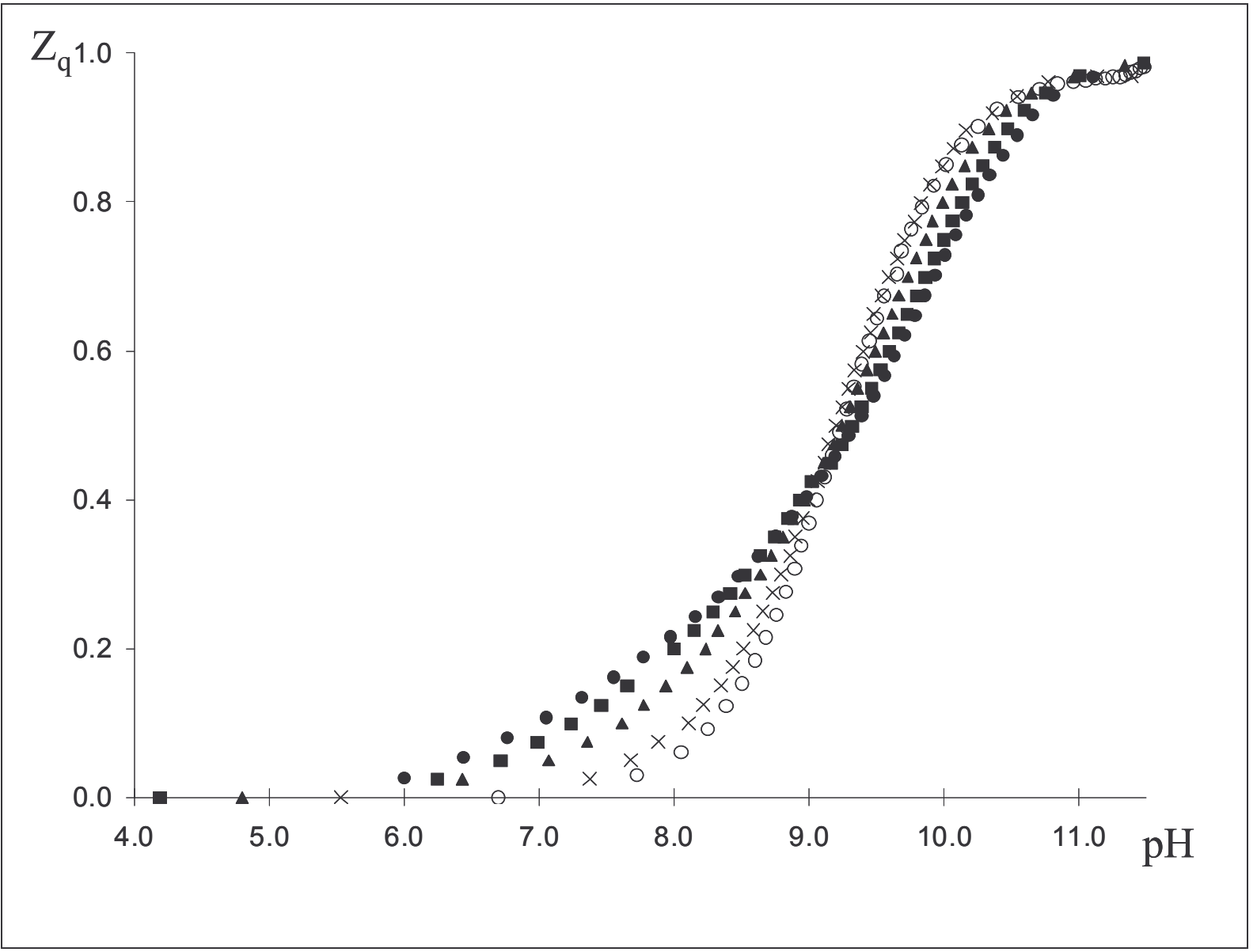

Figura 1.3. Gráfico de $\mathrm{Z}$ como función del $\mathrm{pH}$ para el sistema de $\mathrm{B}(\mathrm{III})-\mathrm{H}_{2} \mathrm{O}$, para diferentes concentraciones de [B(III)]. (O) 0.01M; (X) 0.10M, ( $\mathbf{\Delta}) 0.29 \mathrm{M},(\bullet) 0.50 \mathrm{M},(\bullet) 0.640 \mathrm{M}$. 


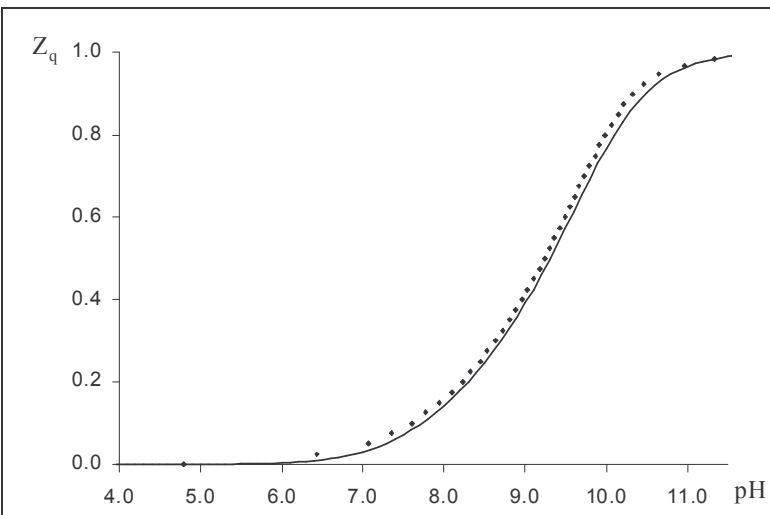

(a)

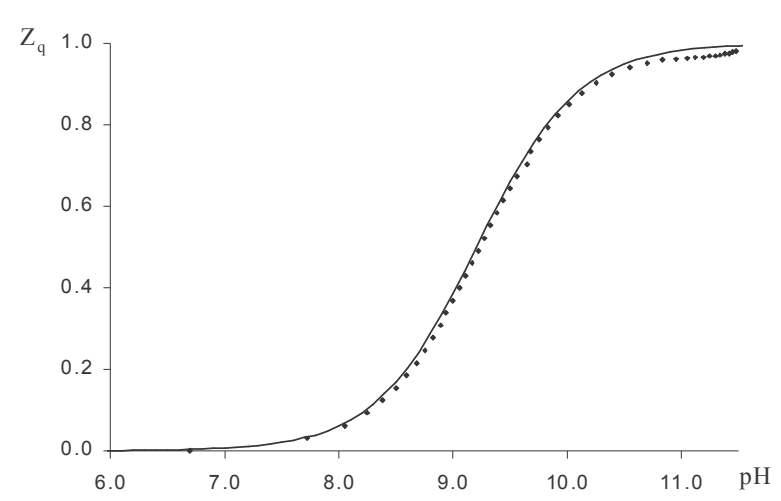

(b)

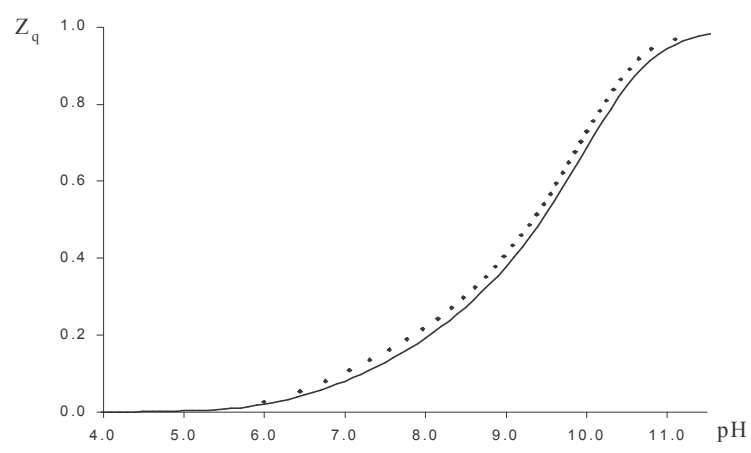

(c)

Figura 1.4. Gráfico de $\mathrm{Z}_{\mathrm{q}}$ como función del $\mathrm{pH}$ para el sistema de $\mathrm{B}(\mathrm{III})-\mathrm{H}_{2} \mathrm{O}$, para diferentes concentraciones de $[\mathrm{B}(\mathrm{III})]_{\mathrm{t}}$. Las líneas continuas representan las simulaciones de $\mathrm{Z}_{\mathrm{q}}$ determinadas a partir del modelo obtenido con SUPERQUAD para este sistema, mientras que los marcadores (घ) representan las $\mathrm{Z}_{\mathrm{q}}$ experimentales. (a) $[\mathrm{B}(\mathrm{III})]_{\mathrm{T}}=0.01 \mathrm{M} ;$ (b) $[\mathrm{B}(\mathrm{III})]_{\mathrm{T}}=0.29 \mathrm{M}$; (c) $[\mathrm{B}(\mathrm{III})]_{\mathrm{T}}=0.645 \mathrm{M}$

\subsubsection{Estudios de RMN}

En las Figuras 1.5 y 1.6 se muestran la familia de espectros de $\mathrm{RMN}$ de ${ }^{11} \mathrm{~B}$ correspondientes a la hidrólisis de soluciones acuosas $0.50 \mathrm{M}$ y $0.70 \mathrm{M}$ de $\mathrm{B}$ (III) 
respectivamente, a partir de $\mathrm{H}_{3} \mathrm{BO}_{3}$ como una función del $\mathrm{pH}$, se puede apreciar que el comportamiento de las señales es similar para ambas concentraciones de $\mathrm{H}_{3} \mathrm{BO}_{3}$. En los espectros de $\mathrm{RMN}$ de ${ }^{11} \mathrm{~B}$ se observa que existe una señal delgada cuyo desplazamiento químico cambia de 20.0 ppm a $0.0 \mathrm{ppm}$, conforme el pH del sistema se incrementa. Por otra

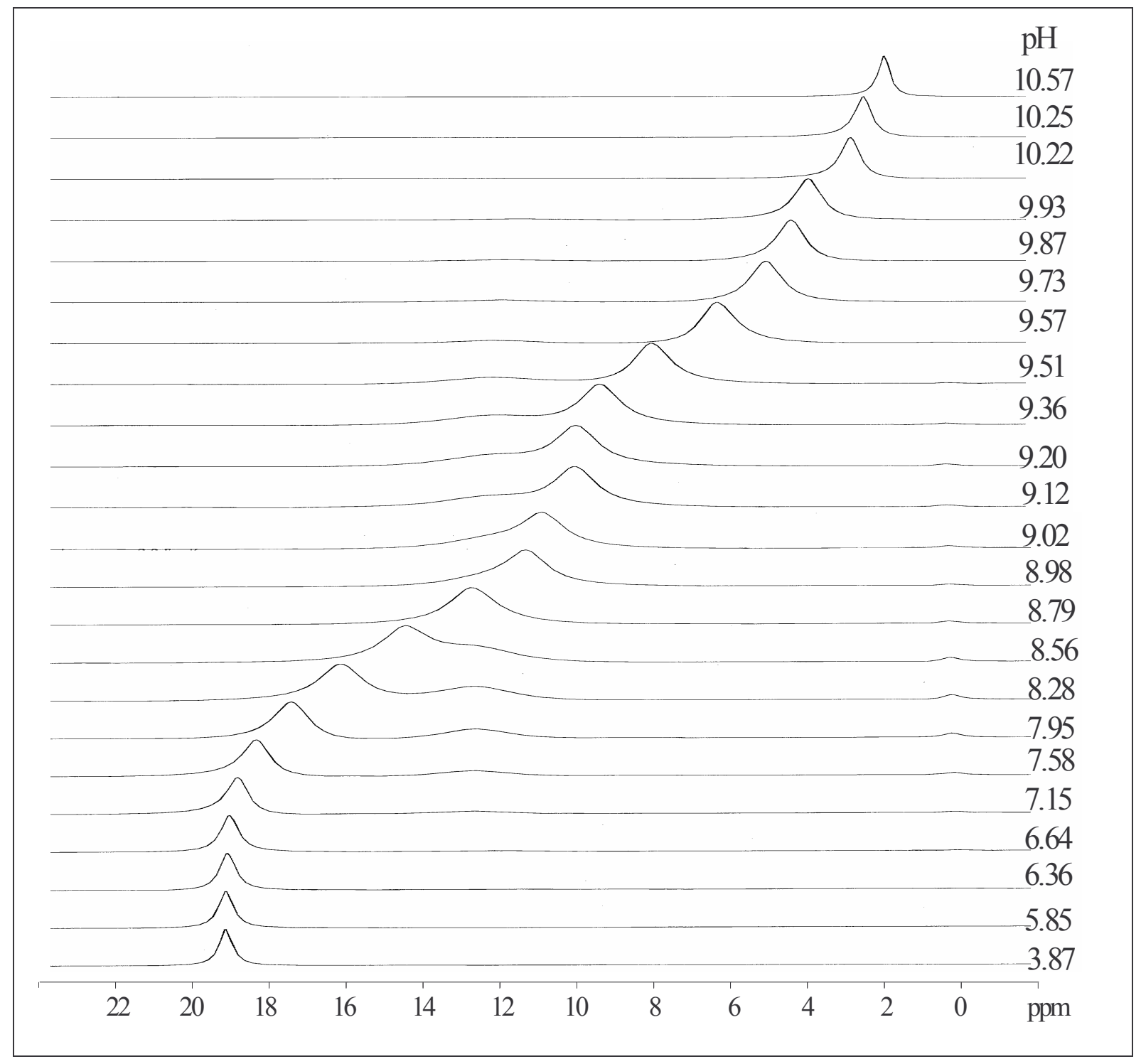

Figura 1.5 Espectros de RMN de ${ }^{11} \mathrm{~B}$ para el sistema $\mathrm{B}(\mathrm{III})-\mathrm{H}_{2} \mathrm{O} . \quad\left[\mathrm{H}_{3} \mathrm{BO}_{3}\right]=0.50 \mathrm{M}$ 
parte, se observan también otras dos señales anchas y de pequeña intensidad (cuyo desplazamiento químico depende ligeramente del $\mathrm{pH}$ ), en $0.90 \mathrm{ppm}$ y $\sim 12.5 \mathrm{ppm}$. Estas aparecen en el intervalo $6.36 \leq \mathrm{pH} \leq 9.51$ y su intensidad aumenta conforme la concentración de B(III) se incrementa en el sistema. El comportamiento descrito para los

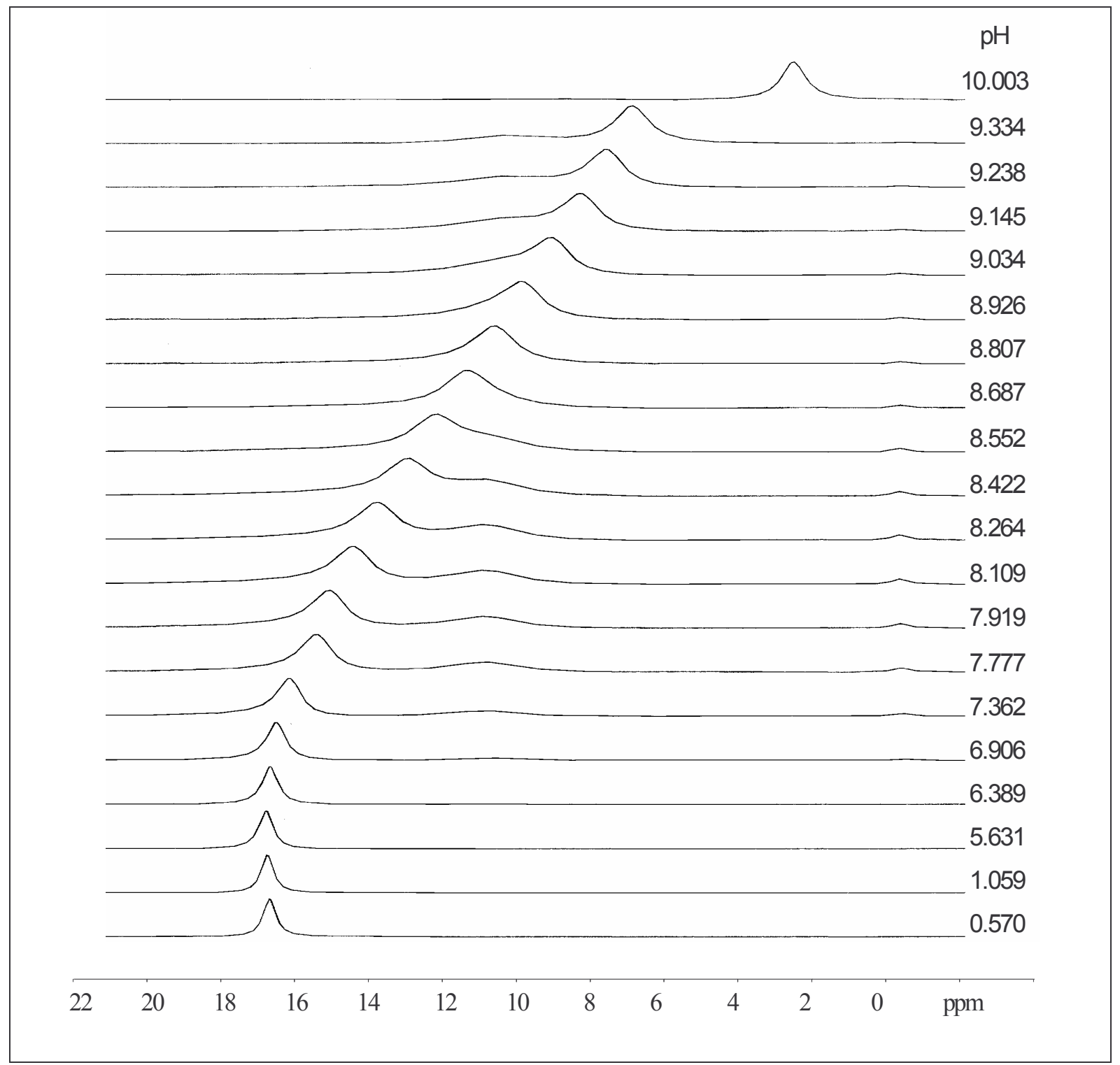

Figura 1.6. Espectros de $\mathrm{RMN}$ de ${ }^{11} \mathrm{~B}$ para el sistema $\mathrm{B}(\mathrm{III})-\mathrm{H}_{2} \mathrm{O} .\left[\mathrm{H}_{3} \mathrm{BO}_{3}\right]=0.70 \mathrm{M}$ 
espectros de ${ }^{11} \mathrm{~B}-\mathrm{RMN}$ sugiere que en el sistema se forman más de dos especies en el proceso de hidrólisis para el sistema $\mathrm{B}(\mathrm{III})-\mathrm{H}_{2} \mathrm{O}$, lo que apoya el modelo utilizado en el tratamiento de los datos potenciométricos.

El desplazamiento de la señal de $20 \mathrm{ppm}$ a $0 \mathrm{ppm}$ puede deberse, como ha sido propuesto anteriormente por Hollins ${ }^{26}$ Guzmán y $\operatorname{Hicks}^{27}$ a un equilibrio rápido con intercambio de protón entre una especie que presenta geometría trigonal $\left(\mathrm{B}(\mathrm{OH})_{3}\right)$ a otra con geometría tetraédrica $\left(\mathrm{B}(\mathrm{OH})_{4}{ }^{-}\right)$de acuerdo al equilibrio entre especies mononucleares mostrado en la ecuación 1.4. Si solamente se tuviera la existencia de especies mononucleares en el sistema el corrimiento en el desplazamiento químico observado $\left(\delta_{0}\right)$ en relación con el $\mathrm{pH}$, se debería únicamente al promedio de las señales del $\mathrm{B}(\mathrm{OH})_{3}\left(\delta_{\mathrm{B}(\mathrm{OH})_{3}}=\right.$ 20ppm) y del $\mathrm{B}(\mathrm{OH})_{4}^{-}\left(\delta_{\mathrm{B}(\mathrm{OH})_{4}}=0 \mathrm{ppm}\right)$ como lo muestra la ecuación 1.5. Para explicar el desplazamiento químico observado para la señal que depende del $\mathrm{pH}$ se emplea un modelo similar al expresado por la ecuación $1.6^{27}$ en la que F representa a las fracciones molares de componente (ver Anexo 1).

$$
\begin{gathered}
\mathrm{B}(\mathrm{OH})_{4}^{-}+\mathrm{H}^{+}=\mathrm{B}(\mathrm{OH})_{3}+\mathrm{H}_{2} \mathrm{O} \quad * \beta_{11}=\frac{1}{\mathrm{~K}_{\mathrm{A}}}=\frac{\left[\mathrm{B}(\mathrm{OH})_{3}\right]}{\left[\mathrm{B}(\mathrm{OH})_{4}^{-}\right]\left[\mathrm{H}^{+}\right]} \\
\delta_{\mathrm{o}}=\mathrm{F}_{\mathrm{B}(\mathrm{OH})_{3}} \delta_{\mathrm{H}_{3} \mathrm{BO}_{3}}+\mathrm{F}_{{\mathrm{B}(\mathrm{OH})_{4}^{-}}_{\mathrm{B}(\mathrm{OH})_{4}^{-}}^{-}} \\
\delta_{o}=\frac{F_{B(\mathrm{OH})_{3}}}{F_{B(\mathrm{OH})_{3}}+F_{B(\mathrm{OH})_{4}^{-}}} \delta_{\mathrm{H}_{3} \mathrm{BO}}+\frac{F_{B(\mathrm{OH})_{4}^{-}}}{F_{B(\mathrm{OH})_{3}}+F_{B(\mathrm{OH})_{4}^{-}}} \delta_{B(\mathrm{OH})_{4}^{-}}
\end{gathered}
$$


Si se combina la ecuación 1.6 con la ecuación de Henderson-Hasselbach y con el balance de las especies mononucleares del boro, puede obtenerse la ecuación 1.7, la cual muestra que el $\mathrm{pH}$ debería ser una función lineal del logaritmo de base decimal del cociente de las diferencias en los desplazamientos químicos, teniendo una pendiente igual a 1 y con un valor de $\mathrm{pK}_{\mathrm{A}}$ igual a la ordenada al origen. Considerando lo anterior se analizaron los datos experimentales correspondientes al desplazamiento de la señal de RMN de ${ }^{11} \mathrm{~B}$, obteniéndose que efectivamente los valores determinados experimentalmente siguen la tendencia lineal esperada, como se muestra en la Figura 1.7, por lo que con base en la ordenada al origen se determinaron los valores de $\mathrm{pK}_{\mathrm{A}}$ del par $\mathrm{B}(\mathrm{OH})_{3} / \mathrm{B}(\mathrm{OH})_{4}{ }^{-}$(Tabla $1.3)$.

$$
p H=p K_{A}+\log \left(\frac{\delta_{B(\mathrm{OH})_{3}}-\delta_{o}}{\delta_{o}-\delta_{B(\mathrm{OH})_{4}^{-}}}\right)
$$

Tabla 1.3. Valores de pKa para $\mathrm{H}_{3} \mathrm{BO}_{3} / \mathrm{B}(\mathrm{OH})_{4}{ }^{-}$determinados por regresión lineal a partir de los cambios en el desplazamiento químico en función del $\mathrm{pH}$ en espectros de RMN de ${ }^{11} \mathrm{~B}$.

\begin{tabular}{|c|c|c|c|}
\hline$[\mathrm{B}(\mathrm{III})]_{\mathrm{total}} / \mathrm{mol} \mathrm{L}^{-1}$ & $\begin{array}{c}\text { Valor de } \\
\mathrm{pK}_{\mathrm{A}}^{\mathrm{a}}\end{array}$ & Pendiente & $\begin{array}{l}\text { Coeficiente de } \\
\text { determinación }\left(\mathrm{r}^{2}\right)\end{array}$ \\
\hline 0.500 & $9.18 \pm 0.007$ & $1.11 \pm 0.007$ & 0.9998 \\
\hline 0.700 & $9.08 \pm 0.024$ & $1.14 \pm 0.019$ & 0.9989 \\
\hline
\end{tabular}


Como se puede apreciar, después de analizar la Tabla 1.3 el valor del pKa determinado concuerda satisfactoriamente con el obtenido por el procesamiento de información potenciométrica (Tabla 1.2). En el tratamiento realizado se puede observar que el valor de la pendiente no es igual a la unidad y que existe un pequeño incremento en el valor de esta de 1.11 a 1.14 , lo cual podría deberse a que en estas concentraciones se tiene la presencia de especies polinucleares por lo que la señal, que depende del $\mathrm{pH}$, también tiene una pequeña contribución de intercambio rápido entre los monómeros y el dimero.

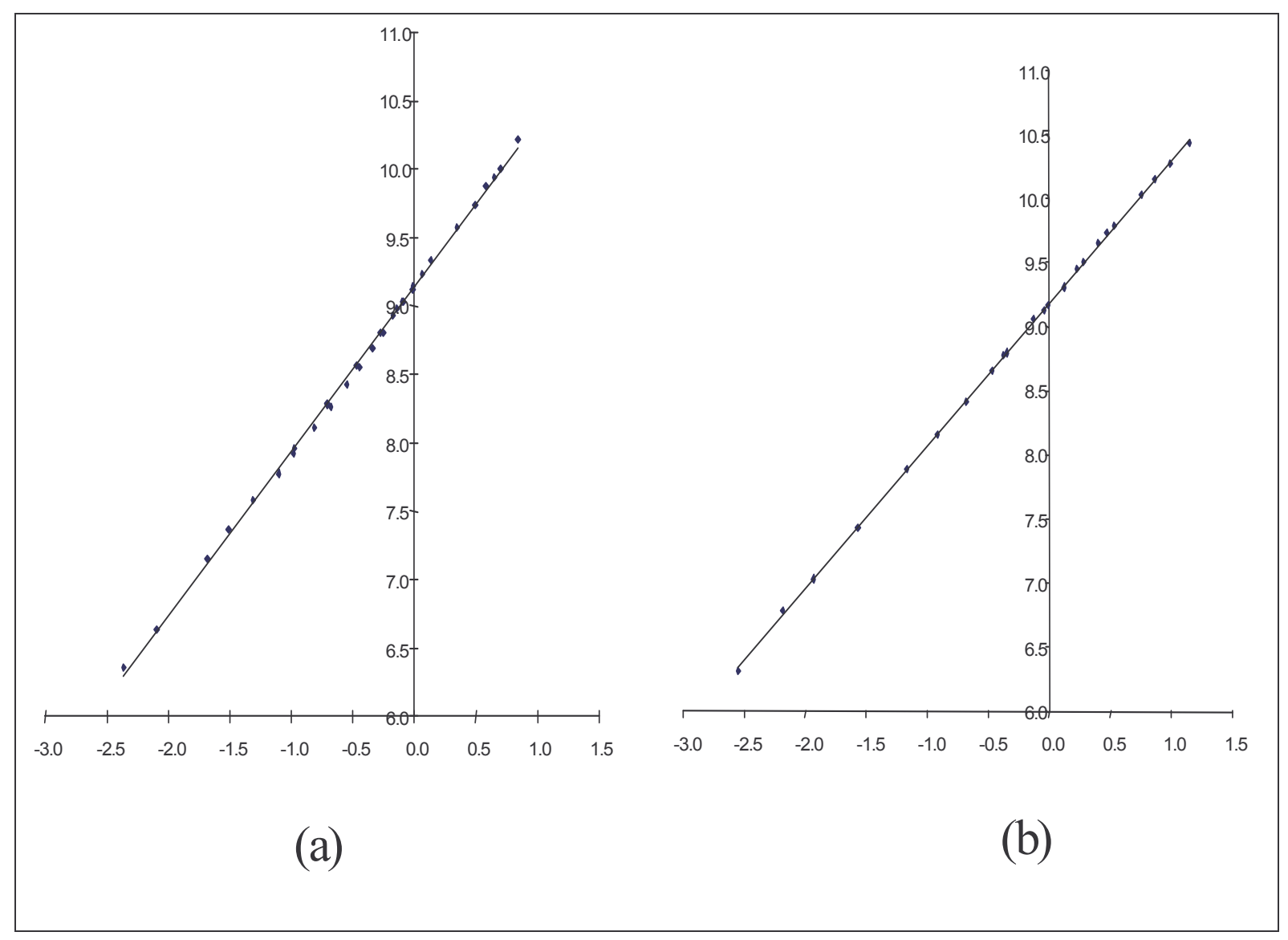

Figura 1.7. Gráficos obtenidos en la determinación del valor de pKa para $\mathrm{H}_{3} \mathrm{BO}_{3} / \mathrm{B}(\mathrm{OH})_{4}{ }^{-}$ determinados por regresión lineal a partir de los cambios en el desplazamiento químico en función del $\mathrm{pH}$ en espectros de $\mathrm{RMN}$ de ${ }^{11} \mathrm{~B}$. Los marcadores son los valores determinados experimentalmente y las líneas representan las curvas de regresión lineal. (a) $\left[\mathrm{H}_{3} \mathrm{BO}_{3}\right]=0.50 \mathrm{M}$, (b) $\left[\mathrm{H}_{3} \mathrm{BO}_{3}\right]=0.70 \mathrm{M}$. 
Como sugieren Ishihara y colaboradores ${ }^{28}$ Sin embargo, este efecto se consideró despreciable para realizar tratamiento de los datos. Por otro lado, se consideró que las dos señales débiles $\left(\delta_{1} \approx 12.50 \mathrm{ppm}\right.$ y $\left.\delta_{2}=0.90 \mathrm{ppm}\right)$ que son independientes del $\mathrm{pH}$ se deben a las especies polinucleares, que por la cercanía de sus desplazamientos químicos y por sus tiempos de relajación se sobreponen.

Si bien generalmente los estudios de RMN de núcleos diferentes a carbono e hidrógeno se han visto encaminados principalmente a la determinación de la geometría de dicho núcleo lo cual permite poder establecer las posibles especies presentes en el sistema, en realidad muy pocos estudios como el anterior han pretendido dar una explicación cuantitativa de las señales. Sin embargo, este estudio solamente proporciona información acerca de las especies mononucleares, lo cual hace que sea limitado.

En este trabajo se desarrolló un algoritmo cuya finalidad es realizar un análisis cuantitativo de las señales de RMN que permita considerar tanto a las especies mononucleares como polinucleares que se pudiesen formar. Dicho algoritmo busca no sólo el proponer un modelo químico (el cual al determinarse por potenciometría suele resultar en ocasiones abstracto) sino que este tenga un sustento en las posibles estructuras de las especies químicas que se están generando en el proceso de hidrólisis. Si bien intuitivamente parece lógico considerar que en un espectro de RMN las fracciones del área bajo cada espectro deberían estar relacionadas con las fracciones molares, hasta donde se sabe esto no ha sido demostrado para el caso de sistemas en donde se presentan al mismo tiempo varias especies en equilibrio. 
Para el desarrollo del algoritmo aquí propuesto se toman como base los valores de las constantes de formación y la estequiometría de las especies propuestas para el sistema en estudio. Con esta información se determinan las fracciones molares tanto de especie como de componente (Apéndice 1) las cuales fueron obtenidas en este trabajo con la ayuda del programa computacional MEDUSA ${ }^{29}$

Por otro lado, se determina el área de cada una de las señales de RMN registradas, lo cual puede realizarse por medio de la integración de la señal o bien cuando se tiene más de una señal asociada se realiza la deconvolución de esta y se realiza su posterior integración, para ello se considera que los factores de respuesta de las señales observadas es la misma para todos los núcleos en todos los ambientes químicos.

Las fracciones calculadas de componente o de especies se agrupan de acuerdo a la posible geometría que tendrán los núcleos dentro de las especies y estas se comparan con los resultados obtenidos del área de cada una de las señales registradas por RMN. Las constantes de formación para el cálculo de las fracciones de componente o especie pueden variarse iterativamente con la finalidad de que estas ajusten con aquellas obtenidas a partir de los espectros de $\mathrm{RMN}^{30}$ Una vez ajustadas las fracciones se comparan con el conjunto de constantes de formación obtenidas para lograr el mejor ajuste con aquellas obtenidas por algún otro método instrumental, lo cual permite dar mayor soporte a un modelo químico propuesto. 
Para la aplicación de este algoritmo en el estudio del sistema $\mathrm{B}(\mathrm{III})-\mathrm{H}_{2} \mathrm{O}$ se consideró agrupar a las fracciones de área experimentales de los espectros de RMN en dos $\operatorname{grupos}^{30}$ uno correspondiente a las especies mononucleares (señales en 0.0ppm y 20.0ppm) y un segundo grupo que considera a las señales debidas a las especies polinucleares (señal en $0.9 \mathrm{ppm}$ y en aproximadamente $12.5 \mathrm{ppm}$ ). Las áreas bajo las señales de los espectros de RMN de ${ }^{11} \mathrm{~B}$ se obtuvieron mediante deconvolución de los espectros.

Las fracciones de área experimentales se compararon tanto con las fracciones de componente como con las fracciones de especie, las cuales se agruparon de la misma forma que las fracciones de área experimentales como lo muestran las ecuaciones 1.8 y 1.9, estas fueron determinadas con ayuda del programa MEDUSA.

$$
\begin{aligned}
& 1=\left(\mathrm{F}_{\mathrm{B}(\mathrm{OH})_{3}}+\mathrm{F}_{\mathrm{B}(\mathrm{OH})_{4}^{-}}\right)+\left(\mathrm{F}_{\mathrm{B}_{2}(\mathrm{OH})_{6}}+\mathrm{F}_{\mathrm{B}_{3}(\mathrm{OH})_{8}^{+}}+\mathrm{F}_{\mathrm{B}_{5}(\mathrm{OH})_{11}^{4+}}\right)=\mathrm{F}_{\text {mono }}+\mathrm{F}_{\text {poli }} \\
& 1=\left(\mathrm{f}_{\mathrm{B}(\mathrm{OH})_{3}}+\mathrm{f}_{\mathrm{B}(\mathrm{OH})_{4}^{-}}\right)+\left(\mathrm{f}_{\mathrm{B}_{2}(\mathrm{OH})_{6}}+\mathrm{f}_{\mathrm{B}_{3}(\mathrm{OH})_{8}^{+}}+\mathrm{f}_{\mathrm{B}_{5}(\mathrm{OH})_{11}^{4+}}\right)=\mathrm{f}_{\text {mono }}+\mathrm{f}_{\text {poli }}
\end{aligned}
$$

Las Figuras 1.8 y 1.9 muestran los mejores ajustes logrados para las señales de ${ }^{11} \mathrm{~B}$ RMN con concentraciones de $\mathrm{B}(\mathrm{III})$ de $0.5 \mathrm{M}$ y $0.7 \mathrm{M}$ respectivamente, con base en ellas se puede establecer que el mejor ajuste se obtiene con las fracciones de las especies en el sistema, y no con las fracciones del componente. Para lo anterior, tanto las fracciones de especie como las de componente se determinaron con base en el modelo obtenido con datos potenciométricos por SUPERQUAD. Con ello se puede apreciar que para el caso de las 
fracciones de componente no se pudo lograr un ajuste del todo satisfactorio, mientras que para el caso de las fracciones molares de especie el ajuste es mucho mejor. Lo anterior lleva a sugerir que el área bajo los picos es directamente proporcional a la concentración de las especies en el sistema y por otro lado, permite establecer que el modelo propuesto en la Tabla 1.2 para el sistema $\mathrm{B}(\mathrm{III})-\mathrm{H}_{2} \mathrm{O}$ es adecuado ya que permite explicar el comportamiento de las señales observadas en $\mathrm{RMN}^{30}$

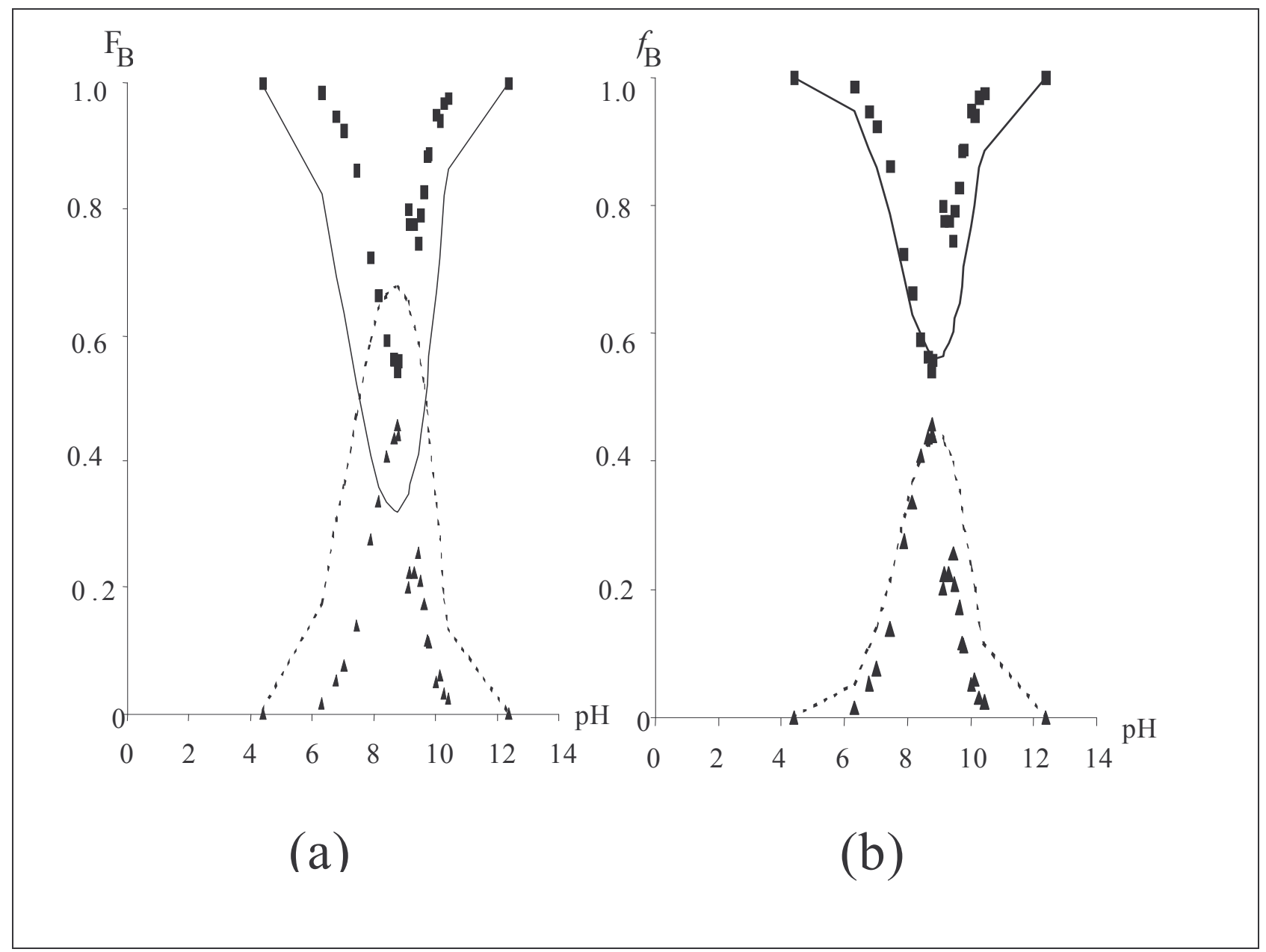

Figura 1.8 (a) Comparación de las fracciones de área experimentales obtenidas de los espectros de $\mathrm{RMN}$ de ${ }^{11} \mathrm{~B}$ con las fracciones molares de componente calculadas a partir del modelo obtenido por SUPERQUAD. (b) Comparación de las fracciones de área experimentales obtenidas de los espectros de RMN de ${ }^{11} \mathrm{~B}$ con las fracciones molares de especie calculadas a partir del modelo obtenido por SUPERQUAD. (ロ) fracciones de área experimentales asignadas a especies mononucleares, $(\boldsymbol{\Delta})$ fracciones de área experimentales asignadas a especies polinucleares, $(-)$ fracciones de área estimadas para especies mononucleares, (- -) fracciones de área estimadas para especies polinucleares. $[\mathrm{B}(\mathrm{III})]$ total $=0.70 \mathrm{M}$. 


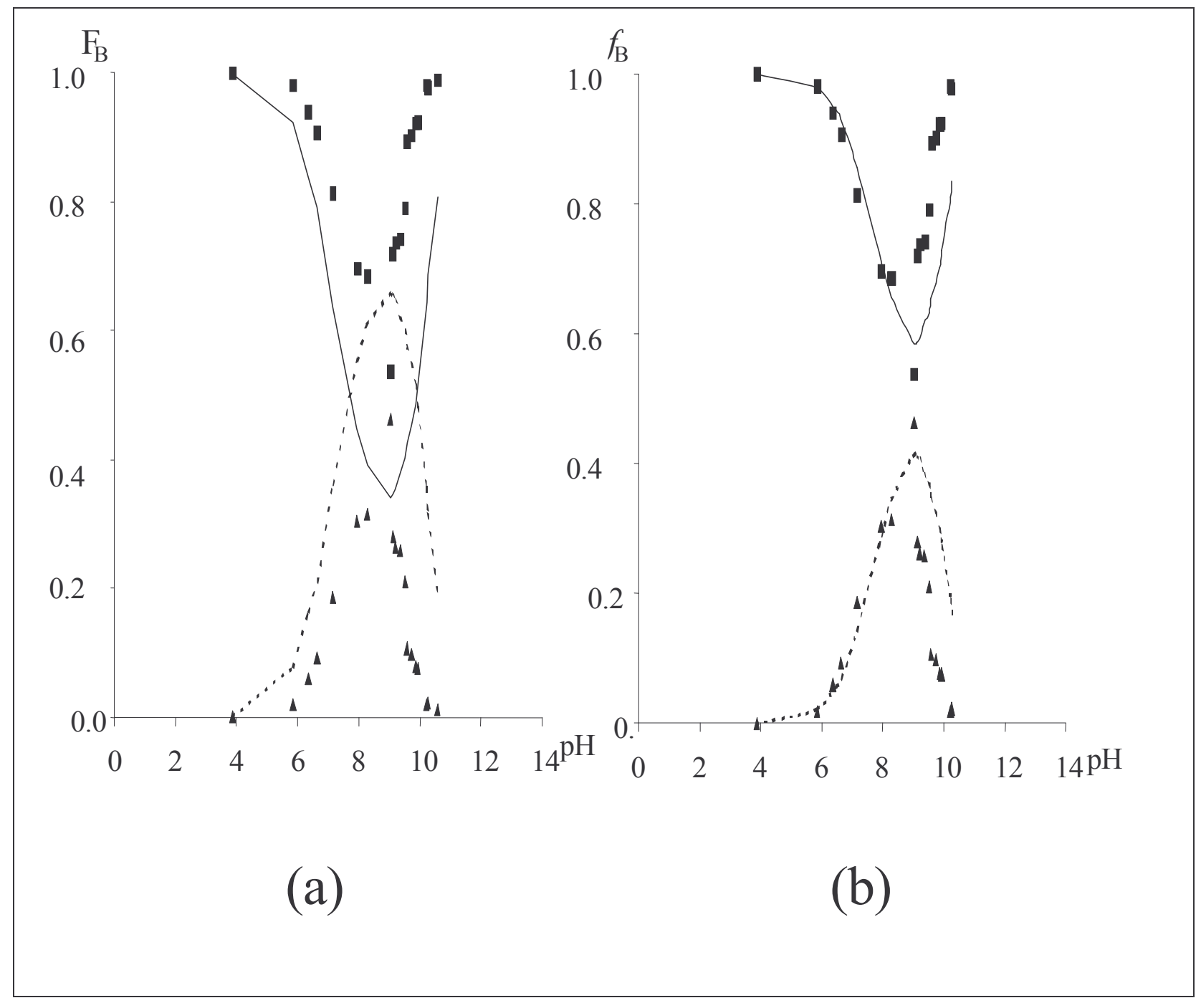

Figura 1.9 (a) Comparación de las fracciones de área experimentales obtenidas de los espectros de RMN de ${ }^{11} \mathrm{~B}$ con las fracciones molares de componente calculadas a partir del modelo obtenido por SUPERQUAD. (b) Comparación de las fracciones de área experimentales obtenidas de los espectros de RMN de ${ }^{11} \mathrm{~B}$ con las fracciones molares de especie calculadas a partir del modelo obtenido por SUPERQUAD. ( $\bullet$ ) fracciones de área experimentales asignadas a mononucleares, $(\mathbf{\Delta})$ fracciones de área experimentales asignadas a polinucleares, $(-)$ fracciones de área estimadas para especies mononucleares, (- -) fracciones de área estimadas para especies polinucleares. [B(III)]total $=0.50 \mathrm{M}$. 


\subsubsection{CONCLUSIONES}

Mediante el análisis y procesamiento de la información potenciométrica se pudo determinar un modelo químico para el sistema $\mathrm{B}(\mathrm{III})-\mathrm{H}_{2} \mathrm{O}$, así como las constantes de formación de las especies químicas. Dicho modelo considera la formación de una especie mononuclear de boro durante el proceso de hidrólisis $\left(\mathrm{B}(\mathrm{OH})_{4}{ }^{-}\right)$con una constante de formación de $-\log * \beta=9.169 \pm 0.011$, además se determinó la existencia de tres especies polinucleares, siendo estas $\mathrm{B}_{2} \mathrm{O}(\mathrm{OH})_{5}{ }^{-}(-\log * \beta=8.553 \pm 0.077), \mathrm{B}_{3} \mathrm{O}_{3}(\mathrm{OH})_{4}{ }^{-}((-\log * \beta=7.607$ $\pm 0.089)$ y $\mathrm{B}_{5} \mathrm{O}_{6}(\mathrm{OH})_{4}{ }^{-}\left(-\log { }^{*} \beta=7.345\right)$. Este modelo pudo ser validado mediante estudios de resonancia magnética nuclear.

Mediante el análisis de las señales de ${ }^{11} \mathrm{~B}$ se pudo determinar el valor de la constante de hidrólisis que lleva a la formación del monómero, el cual coincide con el valor obtenido por potenciometría. Por otro lado, se presenta un nuevo algoritmo que permite obtener información cuantitativa de los espectros de ${ }^{11} \mathrm{~B}-\mathrm{RMN}$, relacionando las áreas de las señales directamente con las fracciones molares de especie. Esto adquiere particular importancia en el área de especiación química ya que permite dar una validación y justificación estructural a un modelo químico, que en muchas ocasiones resultaba abstracto.

Esta relación entre las fracciones de área y la concentración molar de las especies puede estar relacionada con una generalización de la ley de Beer para RMN 


\subsection{SISTEMA $\mathrm{Al}(\mathrm{III})-\mathrm{H}_{2} \mathrm{O}$}

\subsubsection{ANTECEDENTES}

La hidrólisis de aluminio(III) en medio acuoso ha sido objeto de estudio desde hace muchos años, habiendo sido reportadas una gran variedad de especies. Sin embargo existe una gran discrepancia no sólo en cuanto a las especies que se forman sino también en los valores de las constantes de formación asociadas a dichas especies. Numerosos estudios coinciden no sólo en la formación de especies monucleares, sino también en especies polinucleares en solución, las cuales predominantemente se ha establecido que pueden ser una especie polinuclear de bajo peso molecular y una especie con un elevado peso molecular. Sin embargo, la estequiometría de ambas especies no ha sido hasta el momento claramente resuelta. En la Tabla 1.4 se pueden apreciar algunos de los modelos químicos reportados para el proceso de hidrólisis de aluminio(III), en los cuales se pueden observar las diferentes especies reportadas. Los modelos de la Tabla 1.4 permiten ver que se reporta como especie polinuclear de bajo peso molecular la existencia de un dímero o bien la de un trímero; mientras que para el polinuclear de alto peso molecular no es clara la estequiometría. En su gran mayoría los investigadores coinciden en que existe una relación entre aluminio/hidróxido con un valor de aproximadamente 2.4 
Tabla 1.4. Constantes de formación propuestas para la hidrólisis de Al(III

\begin{tabular}{|c|c|c|c|}
\hline AUTOR(ES) & $\begin{array}{l}\text { Especies } \\
\text { Químicas }\end{array}$ & $-\log \beta^{*}$ & Método \\
\hline Schofield R.K. y Taylor A.W.31 & $\mathrm{Al}(\mathrm{OH})^{2+}$ & 4.98 & Potenciometría \\
\hline Ito y Yui N. ${ }^{32}$ & $\mathrm{Al}(\mathrm{OH})^{2+}$ & 5.10 & Potenciometría \\
\hline Aveston $\mathrm{J}^{33}$ & $\begin{array}{l}\mathrm{Al}_{2}(\mathrm{OH})_{2}^{4+} \\
\mathrm{Al}_{13}(\mathrm{OH})_{32}{ }^{7+}\end{array}$ & $\begin{array}{c}7.07(0.06) \\
104.5(0.06)\end{array}$ & Ultracentrifugación \\
\hline Frink C.R. y Peech M. ${ }^{34}$ & $\begin{array}{c}\mathrm{Al}(\mathrm{OH})^{2+} \\
\mathrm{Al}_{2}(\mathrm{OH})_{2}{ }^{4+} \\
\mathrm{Al}_{6}(\mathrm{OH})_{15}{ }^{3+}\end{array}$ & $\begin{array}{c}4.96 \\
7.16 \\
45.29\end{array}$ & Potenciometría \\
\hline Mesmer R. y Baes C.F. ${ }^{1}$ & $\begin{array}{l}\mathrm{Al}_{2}(\mathrm{OH})_{2}{ }^{4+} \\
\mathrm{Al}_{3}(\mathrm{OH})_{4}{ }^{5+} \\
\mathrm{Al}_{14}(\mathrm{OH})_{34}{ }^{8+}\end{array}$ & $\begin{array}{c}7.45(0.17) \\
13.36(0.12) \\
110.45(0.06)\end{array}$ & Potenciometría \\
\hline Turner R. C. ${ }^{35}$ & $\begin{array}{c}\mathrm{Al}(\mathrm{OH})^{2+} \\
\mathrm{Al}_{2}(\mathrm{OH})^{5+} \\
\mathrm{Al}_{2}(\mathrm{OH})_{2}^{4+} \\
\mathrm{Al}_{13}(\mathrm{OH})_{32}{ }^{7+}\end{array}$ & $\begin{array}{c}5.28(0.02) \\
2.96(0.36) \\
6.96(0.10) \\
103.4(0.10)\end{array}$ & Potenciometría \\
\hline $\begin{array}{l}\text { Bottero J.Y., Cases J.M., Flessinger } \\
\text { F. y Poirier J.E. }\end{array}$ & $\begin{array}{l}\mathrm{Al}_{13}(\mathrm{OH})_{36}{ }^{3+} \\
\mathrm{Al}_{2}(\mathrm{OH})_{\mathrm{x}}{ }^{(6-\mathrm{x})}\end{array}$ & $\begin{array}{c}105.0 \\
5.5\end{array}$ & $\begin{array}{l}\text { Potenciometría y } \\
\text { RMN }\end{array}$ \\
\hline Öhman L. y Forsling W. ${ }^{8}$ & $\begin{array}{c}\mathrm{Al}(\mathrm{OH})^{2+} \\
\mathrm{Al}_{3}(\mathrm{OH})_{4}{ }^{5+} \\
\mathrm{Al}_{13}(\mathrm{OH})_{32}{ }^{7+}\end{array}$ & $\begin{array}{c}5.52(0.04) \\
13.57(0.12) \\
109.2(0.12)\end{array}$ & Potenciometría \\
\hline $\begin{array}{l}\text { Charlet, Deloume J.P., Duc G. y } \\
\text { Thomas-David G. }^{36}\end{array}$ & $\begin{array}{c}\mathrm{Al}(\mathrm{OH})^{2+} \\
\mathrm{Al}_{2}(\mathrm{OH})_{5}^{+} \\
\mathrm{Al}_{4}(\mathrm{OH})_{10}{ }^{2+} \\
\mathrm{Al}_{6}(\mathrm{OH})_{15}{ }^{3+}\end{array}$ & $\begin{array}{c}4.70-5.00 \\
19.40-20.30 \\
37.30 \\
53.00-53.52\end{array}$ & Potenciometría \\
\hline
\end{tabular}


Tabla 1.4. Constantes de formación propuestas para la hidrólisis de Al(III) (Continuación)

\begin{tabular}{|c|c|c|c|}
\hline AUTOR(ES) & $\begin{array}{l}\text { Especies } \\
\text { Químicas }\end{array}$ & $-\log \beta^{*}$ & Método \\
\hline Tóth I., Zékány L. y Brücher E. ${ }^{37}$ & $\begin{array}{l}\mathrm{Al}(\mathrm{OH})^{2+} \\
\mathrm{Al}(\mathrm{OH})_{2}^{+} \\
\mathrm{Al}(\mathrm{OH})_{3} \\
\mathrm{Al}(\mathrm{OH})_{4}^{-}\end{array}$ & $\begin{array}{l}5.5(0.6) \\
9.8(0.1) \\
15.0(0.1) \\
23.4(0.1)\end{array}$ & Potenciometría \\
\hline Ares Jorge ${ }^{38}$ & $\begin{array}{c}\mathrm{Al}(\mathrm{OH})^{2+} \\
\mathrm{Al}(\mathrm{OH})_{2}^{+} \\
\mathrm{Al}(\mathrm{OH})_{3} \\
\mathrm{Al}(\mathrm{OH})_{4}^{-} \\
\mathrm{Al}_{2}(\mathrm{OH})_{2}^{4+} \\
\mathrm{Al}_{3}(\mathrm{OH})_{4}^{5+}\end{array}$ & $\begin{array}{c}5.0 \\
10.1 \\
15.0 \\
22.1 \\
7.0 \\
13.9\end{array}$ & Potenciometría \\
\hline Brown P. y Sylva R. ${ }^{39}$ & $\begin{array}{c}\mathrm{Al}(\mathrm{OH})^{2+} \\
\mathrm{Al}(\mathrm{OH})_{2}{ }^{+} \\
\mathrm{Al}_{3}(\mathrm{OH})_{4}{ }^{5+} \\
\mathrm{Al}_{13}(\mathrm{OH})_{32}{ }^{7+}\end{array}$ & $\begin{array}{c}5.35(0.01) \\
10.82(0.02) \\
13.13(0.005) \\
107.47(0.04)\end{array}$ & Potenciometría \\
\hline Letterman R.D. y Asolekar S.R: ${ }^{40}$ & $\begin{array}{c}\mathrm{Al}(\mathrm{OH})^{2+} \\
\mathrm{Al}_{14}(\mathrm{OH})_{32}{ }^{7+}\end{array}$ & $\begin{array}{l}4.4 \\
103\end{array}$ & Potenciometría \\
\hline Marklund E. y Öhman L. ${ }^{41}$ & $\mathrm{Al}_{13}(\mathrm{OH})_{32}{ }^{7+}$ & $-105.5(0.20)$ & $\begin{array}{c}\text { Potenciometría y } \\
\text { RMN }\end{array}$ \\
\hline
\end{tabular}

Por otro lado, Akitt y colaboradores ${ }^{42-47}$ realizado diversos estudios de Resonancia Magnética Nuclear (RMN) para determinar a las posibles especies que se forman durante la hidrólisis de aluminio(III). Sin embargo ninguno de dichos estudios han podido establecer contundentemente cual es el mejor modelo para este sistema en estudio. 
En un trabajo realizado por este mismo grupo de trabajo ${ }^{48}$ mediante estudios de potenciometría y de Resonancia Magnética Nuclear se propuso un modelo químico y sus respectivas constantes de formación para el sistema $\mathrm{Al}(\mathrm{III})-\mathrm{H}_{2} \mathrm{O}$ en el que se estableció que durante el proceso de hidrólisis se formaban las especies $\mathrm{Al}(\mathrm{OH})^{2+}(5.29(0.04)), \mathrm{Al}_{3}(\mathrm{OH})_{4}{ }^{5+}$ (13.57 (0.05)) y $\mathrm{Al}_{13}(\mathrm{OH})_{32}{ }^{7+}(110.54(0.10))$ modelo que fue corroborado Pacheco ${ }^{49}$ quien determinó además que a relativamente bajas concentraciones de $\mathrm{Al}(\mathrm{III})$ se forma la especie monomérica $\mathrm{Al}(\mathrm{OH})_{3}$ con una constante de formación de $12.96 \pm 0.02$

\subsubsection{EXPERIMENTAL}

\subsubsection{Estudios Conductimétricos}

Al menos dos muestras de soluciones de Al(III), preparadas a partir de $\mathrm{Al}\left(\mathrm{NO}_{3}\right)_{3} \cdot 9 \mathrm{H}_{2} \mathrm{O}$ (Sigma ACS, $99.1 \%$ ), en un intervalo de $5.65 \times 10^{-3} \mathrm{M}$ a $28.23 \times 10^{-3} \mathrm{M}$ fueron valoradas conductimétricamente con soluciones estandarizadas de hidróxido de sodio, preparadas como se indicó en la sección 1.1.2.1. Las lecturas de conductividad se realizaron con un conductimetro Radiometer Copenhagen CDM230 equipado con una celda conductimétrica de cuatro polos (con cuatro anillos de platino) Radiometer Copenhagen CDC865, calibrándose el equipo antes de cada valoración con soluciones estándar de $\mathrm{NaCl}$.

Durante la experimentación se mantuvo un flujo constante de nitrógeno de alta pureza para evitar la carbonatación y la temperatura se mantuvo constante a $25.0^{\circ} \mathrm{C}(\Delta \mathrm{T} \pm$ 
$0.1{ }^{\circ} \mathrm{C}$ ) por medio de un baño de agua (VWR PolyScience modelo 12101) y empleándose celdas de vidrio de doble camisa. El agua empleada en todos los experimentos y en la preparación de la soluciones fue agua desionizada y descarbonatada Tipo 1 (Millipore).

\subsubsection{Estudios de Resonancia Magnética Nuclear (RMN)}

Se preparó una solución acuosa de $\mathrm{Al}(\mathrm{III})$ de concentración $1.00 \mathrm{M}$ a partir de $\mathrm{Al}\left(\mathrm{NO}_{3}\right)_{3}{ }^{\bullet} 9 \mathrm{H}_{2} \mathrm{O}$ (Sigma ACS 99.1\%). Se hidrolizó mediante la adición de solución acuosa de hidróxido de sodio estandarizado, hasta una relación $\mathrm{OH} / M$ igual a 0.5 y esta fue diluida sucesivamente con agua desionizada hasta alcanzar una concentración de $0.001 \mathrm{M}$, registrándose el $\mathrm{pH}$ para todas las soluciones hidrolizadas.

Los espectros de RMN de ${ }^{27} \mathrm{Al}$ se obtuvieron a $25{ }^{\circ} \mathrm{C}$ con un espectrofotometro Brucker DMX-500 empleando $\mathrm{Al}\left(\mathrm{H}_{2} \mathrm{O}\right)_{6}{ }^{3+}$ como referencia de desplazamiento químico, la excitación empleada fue de 130.32MHz.

\subsubsection{RESULTADOS Y ANÁLISIS}

Como ya se había mencionado anteriormente, este sistema se estudió previamente ${ }^{48,49}$, determinándose mediante estudios de potenciometría el modelo que se muestra en la Tabla 1.5. En dicha tabla se puede apreciar la formación de dos especies monoméricas $\left(\mathrm{Al}(\mathrm{OH})^{2+}\right.$ y $\left.\mathrm{Al}(\mathrm{OH})_{3}\right)$, así como la de un polinuclear de bajo peso molecular $\left(\mathrm{Al}_{3}(\mathrm{OH})_{4}{ }^{5+}\right)$ y uno de alto peso molecular $\left(\mathrm{Al}_{13}(\mathrm{OH})_{32}{ }^{7+}\right)$. Considerando este modelo 
químico en el presente trabajo se realizaron estudios encaminados a la confirmación de estas especies y sus constantes de formación, mediante estudios de conductimetría y la aplicación del algoritmo desarrollado para analizar cuantitativamente las señales de RMN de ${ }^{27} \mathrm{Al}$, con el propósito de contar con un modelo químico confiable y que esté corroborado por diversas técnicas analíticas. Como se mencionó anteriormente para este sistema existen diversos modelos, sin embargo, hay una gran divergencia tanto en las especies como en las constantes reportadas, lo cual dificulta la interpretación química de este.

Tabla 1.5 Valores de las constantes de formación para el mejor refinamiento obtenido a partir de datos potenciométricos procesados con SUPERQUAD para el sistema $\mathrm{Al}(\mathrm{III})-\mathrm{H}_{2} \mathrm{O}$.

\begin{tabular}{ccc}
\hline Especies & $-\log * \beta$ & Parámetros \\
& & Estadísticos \\
\hline $\mathrm{Al}(\mathrm{OH})^{2+}$ & $5.29^{a}$ & $\sigma=1.120$ \\
$\mathrm{Al}(\mathrm{OH})_{3}$ & $12.96 \pm 0.02$ & $\mathrm{U}=108.03$ \\
$\mathrm{Al}_{3}(\mathrm{OH})_{4}{ }^{5+}$ & $13.50 \pm 0.02$ & $\chi^{2}=7.45$ \\
$\mathrm{Al}_{13}(\mathrm{OH})_{32}{ }^{7+}$ & $109.70 \pm 0.09$ & \\
\hline${ }^{\mathrm{a}}$ Valor constante durante el refinamiento & &
\end{tabular}

\subsubsection{Estudios Conductimétricos}

Las valoraciones conductimétricas para sistemas de $\mathrm{Al}(\mathrm{III})-\mathrm{H}_{2} \mathrm{O}$ se muestran en la Figura 1.10, las cuales se llevaron a cabo hasta alcanzar la relación $\mathrm{OH} / \mathrm{Al}=2.5$, ya que 
después de esta relación tenía lugar la precipitación en el sistema. Se puede observar que la conductividad permanece casi constante a lo largo de toda la valoración dentro del intervalo de concentraciones de $6.0\left(10^{-3}\right) \mathrm{M} \leq[\mathrm{Al}(\mathrm{III})]_{\text {total }} \leq 2.8\left(10^{-2}\right) \mathrm{M}$ (Figura $\left.1.10 \mathrm{a}\right)$, mientras que para una concentración de $[\mathrm{Al}(\mathrm{III})]=0.12 \mathrm{M}$ se puede apreciar una ligera disminución de la conductividad hasta una relación $\mathrm{OH} / \mathrm{Al}$ de 0.2 y posteriormente esta se incrementa conforme el grado de hidrólisis progresa (Figura 1.10b).

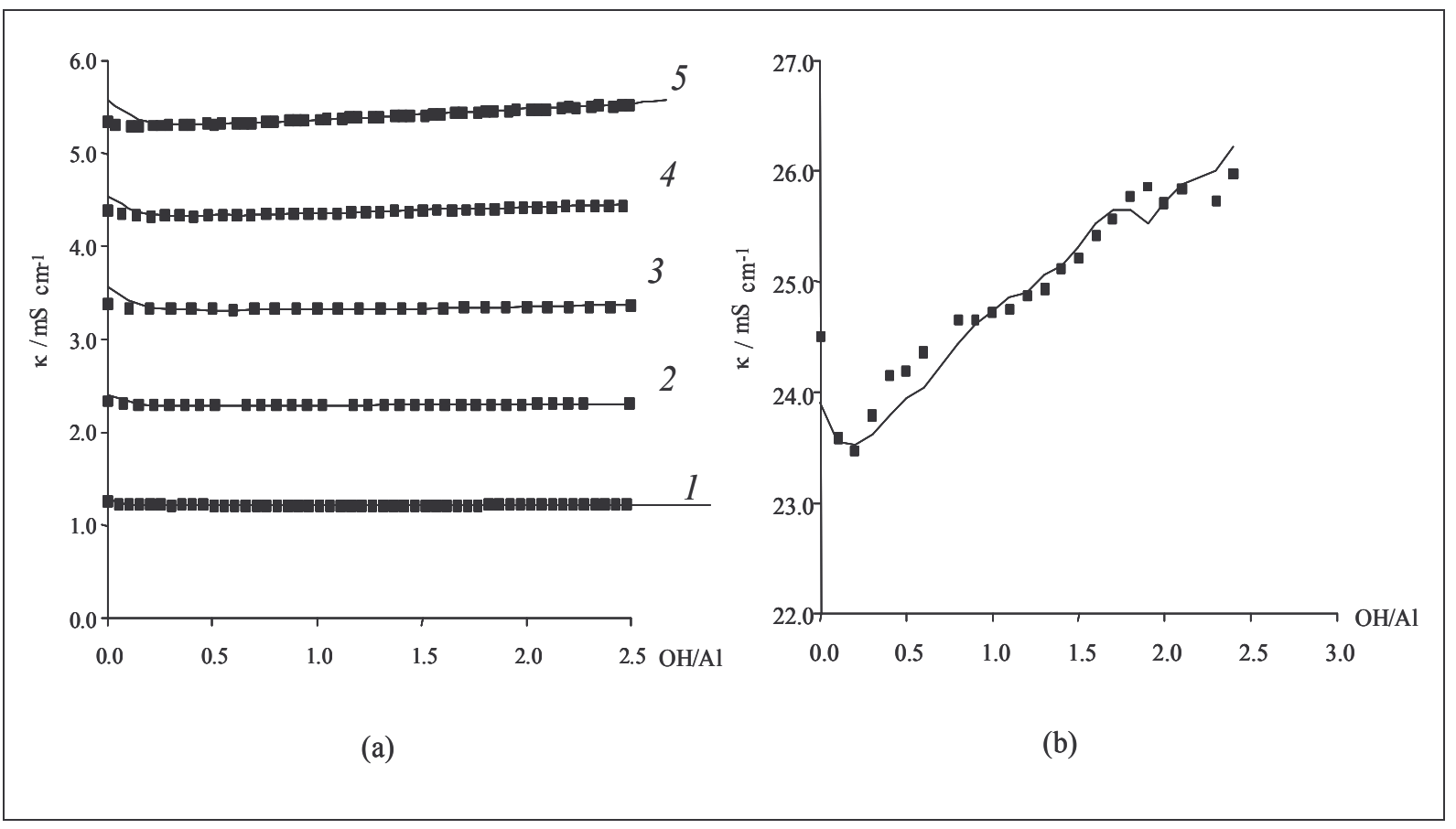

Figura 1.10. Valoraciones conductimétricas para el sistema $\mathrm{Al}(\mathrm{III})-\mathrm{H}_{2} \mathrm{O}$.

(a) (1) $[\mathrm{Al}(\mathrm{III})]_{\text {total }}=6.0\left(10^{-3}\right) \mathrm{M}$; (2) $[\mathrm{Al}(\mathrm{III})]_{\text {total }}=11.0\left(10^{-3}\right) \mathrm{M}, \quad$ (3) $[\mathrm{Al}(\mathrm{III})]_{\text {total }}=16.0\left(10^{-3}\right) \mathrm{M}$, (4) $[\mathrm{Al}(\mathrm{III})]_{\text {total }}=22.0\left(10^{-3}\right) \mathrm{M}, \quad(5) \quad[\mathrm{Al}(\mathrm{III})]_{\text {total }}=28.0\left(10^{-3}\right) \mathrm{M} ; \quad$ (b). $\quad[\mathrm{Al}(\mathrm{III})]_{\text {total }}=0.12 \mathrm{M}$. Las líneas representan la conductividad estimada empleando el modelo obtenido en la Tabla 1.5 por medio del programa SUPERQUAD. Los marcadores representan los valores de conductividad experimentales

Con la finalidad de validar el modelo propuesto en la Tabla 1.5 se compararon los valores de conductividad equivalente obtenidos experimentalmente con los valores de conductividad calculados para las especies $\mathrm{Al}_{\mathrm{i}}(\mathrm{OH})_{\mathrm{j}}{ }^{3 \mathrm{i}-\mathrm{j}}$, con base en la ecuación 1.10. 


$$
\begin{aligned}
\kappa= & |z| \lambda_{\mathrm{Na}^{+}}\left[\mathrm{Na}^{+}\right]+|z| \lambda_{\mathrm{NO}_{3}^{-}}\left[\mathrm{NO}_{3}^{-}\right]+|z| \lambda_{\mathrm{H}^{+}}\left[\mathrm{H}^{+}\right]+|z| \lambda_{\mathrm{OH}}\left[\mathrm{OH}^{-}\right]+ \\
& \sum|z| \lambda_{A l_{i}(\mathrm{OH})_{j}^{3 i-j}} F_{\mathrm{Al}_{i}(\mathrm{OH})_{j}^{3 i-j}}[\mathrm{Al}(\mathrm{III})]_{\mathrm{total}}
\end{aligned}
$$

en donde $\mathrm{z}$ representa la carga de las especies y $F_{A l i(O H) j 3 i-j}$ es la fracción molar de componente (Apéndice 1) para cada especie de Al(III), calculadas por medio del programa computacional MEDUSA. La concentración de $\mathrm{Na}^{+}$fue determinada con base en la cantidad de hidróxido de sodio adicionado para la hidrólisis, mientras que la concentración de $\mathrm{NO}_{3}{ }^{-}$se estimó en función de la cantidad inicial de $\mathrm{Al}\left(\mathrm{NO}_{3}\right)_{3}$. Por otro lado, la concentración de $\mathrm{H}^{+}$y $\mathrm{OH}^{-}$se determinó a partir del $\mathrm{pH}$ experimental registrado, y los coeficientes de conductividad equivalente (1) se estimaron mediante iteración. La Tabla 1.6 muestra los coeficientes de conductividad equivalente estimados para cada especie, es importante destacar que los coeficientes de conductividad equivalente se estimaron para cada concentración, y que estos incrementaron su valor conforme la concentración disminuía, lo cual es un comportamiento esperado debido a que la conductividad equivalente es directamente proporcional a la movilidad de la especie ${ }^{50}$.

En la misma Figura 1.10 se muestran los valores de las conductividades estimadas, se puede apreciar que existe un adecuado ajuste de estas con los valores obtenidos experimentalmente, con lo cual se puede establecer la validez del modelo propuesto en la Tabla 1.5, determinado para el sistema $\mathrm{Al}(\mathrm{III})-\mathrm{H}_{2} \mathrm{O}$ por SUPERQUAD, ya que este puede explicar satisfactoriamente el comportamiento experimental de las curvas conductimétricas. 
Tabla 1.6. Valores estimados de Conductividad equivalente para el ajuste de las curvas de valoración conductimétricas para el sistema $\mathrm{Al}(\mathrm{III})-\mathrm{H}_{2} \mathrm{O}$.

\begin{tabular}{ccc}
\hline Sistema & Especies & $\lambda / \mathrm{mS} \mathrm{cm}^{2}$ equivalente $^{-1}$ \\
\hline & $\mathrm{Al}^{3+}$ & $3.5-15$ \\
$\mathrm{Al}(\mathrm{OH})^{2+}$ & 10 \\
$\mathrm{Al}(\mathrm{OH})_{3}$ & 0 \\
$\mathrm{Al}(\mathrm{III})-\mathrm{H}_{2} \mathrm{O}$ & $\mathrm{Al}_{3}(\mathrm{OH})_{4}{ }^{5+}$ & 8 \\
& $\mathrm{Al}_{13}(\mathrm{OH})_{32}{ }^{7+}$ & $42-47$ \\
& $\mathrm{Na}^{+}$ & $8.6-18$ \\
& $\mathrm{NO}_{3}{ }^{-}$ & $50-70.1$ \\
\hline
\end{tabular}

\subsubsection{Estudios de RMN}

En la Figura 1.11 se pueden observar los espectros de RMN para ${ }^{27} \mathrm{Al}$ obtenidos por Pacheco $^{44}$. En ellos se puede apreciar que en un intervalo de $3.04 \leq \mathrm{pH} \leq 4.55$ existe un pico en 0 ppm el cual se ha relacionado con una geometría octaédrica, asignándose a las especies de $\mathrm{Al}^{3+}, \mathrm{Al}(\mathrm{OH})^{2+}$ y $\mathrm{Al}(\mathrm{OH})_{3}$, ya que estas especies presentan un núcleo de $\mathrm{Al}(\mathrm{III})$ con esta geometría. Por otro lado, en el intervalo de $3.47 \leq \mathrm{pH} \leq 3.94$ se observa otra señal en $5 \mathrm{ppm}$ (figura 1.12) la cual se ha establecido que está relacionada con la especie polinuclear de bajo peso molecular (oligomero, $\mathrm{Al}_{3}(\mathrm{OH})_{4}{ }^{5+}$ ). En el intervalo de $3.61 \leq \mathrm{pH} \leq 6.32$ se aprecia una señal en $63 \mathrm{ppm}$ la cual se estableció está relacionada con la especies $\mathrm{Al}_{13}(\mathrm{OH})_{32}{ }^{7+}$. Dicha señal desaparece en un intervalo de $5.45 \leq \mathrm{pH} \leq 6.32$ lo cual se justificó que es a consecuencia de la formación de especies de poca simetría o bien debido a la precipitación de $\mathrm{Al}(\mathrm{OH})_{3(\mathrm{~s})}$. 
Con la finalidad de confirmar el modelo propuesto en la Tabla 1.5 se utilizó el algoritmo antes mencionado ${ }^{30}$ (sección 1.1.3.2), para lo cual se determinó el área, mediante integración, para cada señal registrada en los espectros de ${ }^{27} \mathrm{Al}-\mathrm{RMN}$. Las fracciones molares de componente y de especie se calcularon con ayuda del programa computacional MEDUSA empleando las constantes de formación que se presentan en la Tabla 1.5. Para la

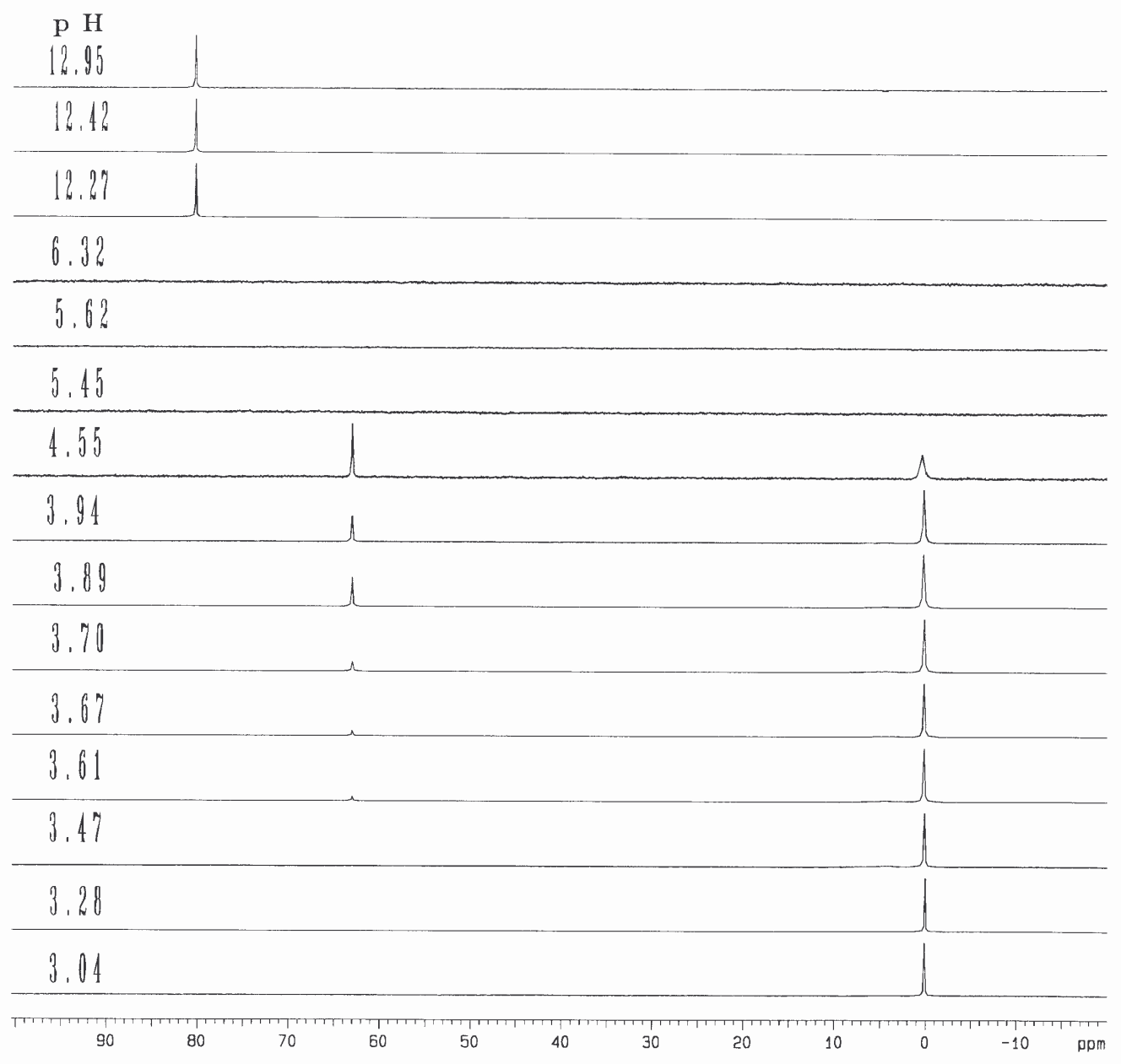

Figura 1.11. Espectros de $\mathrm{RMN}$ para ${ }^{27} \mathrm{Al}$ con diferentes grados de hidrólisis para una concentración de $[\mathrm{Al}(\mathrm{III})]_{\text {total }}=0.500 \mathrm{M}$. . (tomados de la tesis de Pacheco L. ${ }^{44}$ ) 


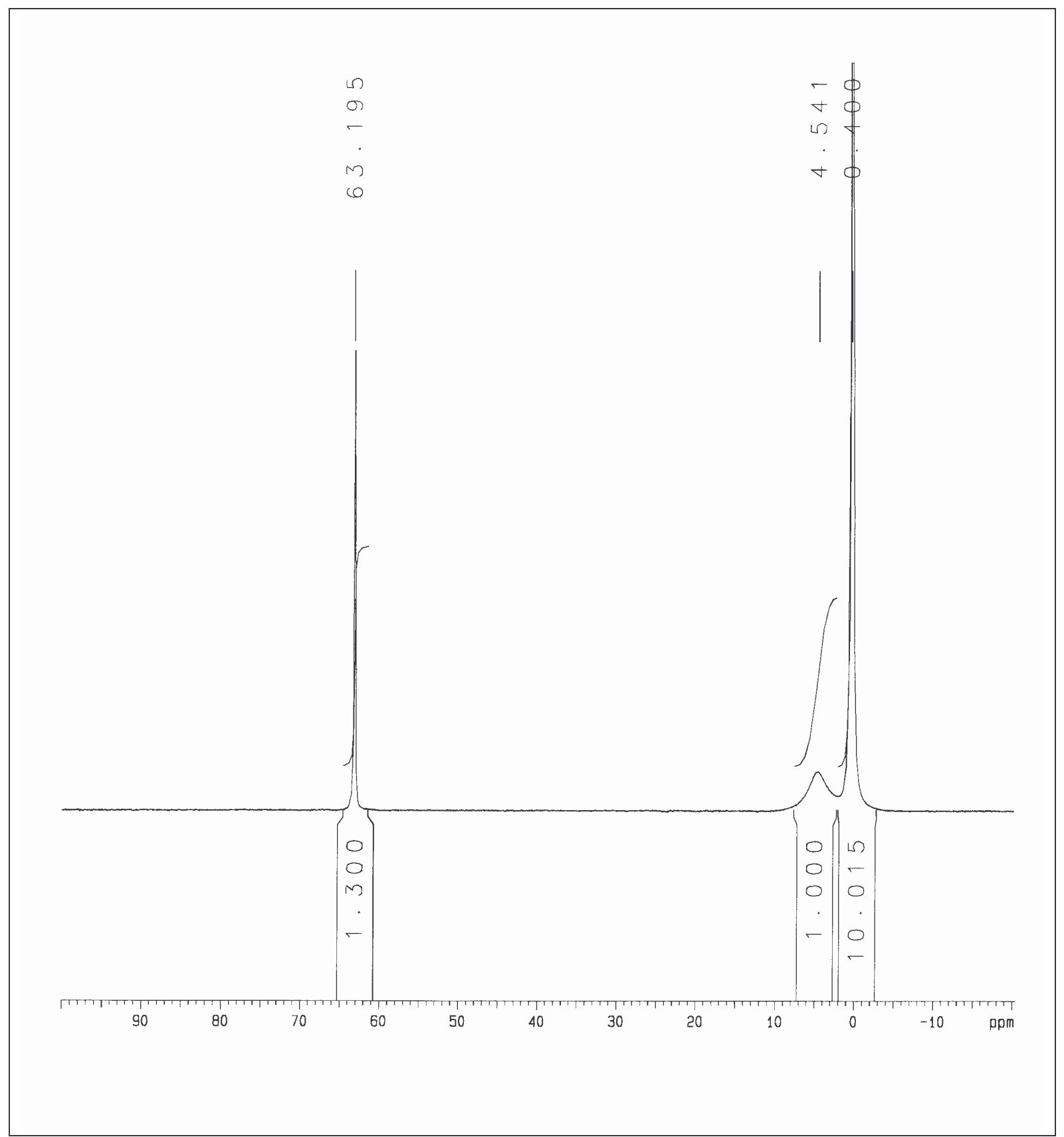

Figura 1.12. Espectros de RMN para ${ }^{27} \mathrm{Al}$ para una solución de concentración de $[\mathrm{Al}(\mathrm{III})]_{\text {total }}=$ $0.500 \mathrm{M}$, con $\mathrm{pH}=3.67$; en esta se pueden apreciar claramente la señal en 0 ppm, 5 ppm y 63 ppm. (tomados de la tesis de Pacheco L. ${ }^{44}$ )

comparación de fracciones de área de las señales de RMN con aquellas determinadas a partir del modelo químico se consideraron dos posibilidades. En un primer modelo se considera que dado que la especie $\mathrm{Al}_{13}(\mathrm{OH})_{32}{ }^{7+}$ al tener una estructura tipo Keggin posee un aluminio central con geometría tetraédrica y doce aluminios octaédricos rodeándolo, 
entonces la fracción de área relacionada con la señal de ${ }^{27} \mathrm{Al}-\mathrm{RMN}$ en $0.0 \mathrm{ppm}$ se debe a las especies mononucleares de $\mathrm{Al}(\mathrm{III})\left(\mathrm{Al}^{3+}, \mathrm{Al}(\mathrm{OH})^{2+}\right.$ y $\left.\mathrm{Al}(\mathrm{OH})_{3}\right)$. En este caso los núcleos de aluminio deberán exhibir una geometría octaédrica, así como una porción ponderada de la fracción correspondiente (ya sea de especie o de componente) de la especie $\mathrm{Al}_{13}(\mathrm{OH})_{32}{ }^{7+}$. La fracción de área correspondiente a la señal de 5ppm se le asigna al oligómero $\mathrm{Al}_{3}(\mathrm{OH})_{4}{ }^{5+}$, mientras que el área de la señal de $63 \mathrm{ppm}$ se estableció que se debe a núcleos de aluminio con geometría tetraédrica por lo que se comparó con la parte proporcional de la fracción, de especie o componente, de $\mathrm{Al}_{13}(\mathrm{OH})_{32}{ }^{7+}$ (de acuerdo a lo antes mencionado)

En un segundo modelo se tomó en consideración la asignación de acuerdo a lo reportado por Akitt y colaboradores ${ }^{42-47}$ quienes establecen que la señal en 0 ppm se debe únicamente a las especies mononucleares de aluminio(III), mientras que la señal en 5ppm esta relacionada con el polinuclear de bajo peso molecular $\mathrm{Al}_{3}(\mathrm{OH})_{4}{ }^{5+}$ y finalmente la señal en 63 ppm se relaciona con la $\mathrm{Al}_{13}(\mathrm{OH})_{32}{ }^{7+}$. Se puede considerar en este caso dos posibles aspectos: uno de ellos apunta a que ésta señal se debe únicamente al núcleo tetraédrico del aluminio en la especie y que los doce aluminios restantes son "invisibles" debido a una distorsión en el ambiente químico o bien que los tiempos de relajación para estos son demasiado rápidos ${ }^{42}$. La segunda consideración es el sugerir que esta señal en $63 \mathrm{ppm}$ se debe de alguna manera tanto al núcleo tetraédrico como a los doce octaédricos dentro de la especie correspondiente. 


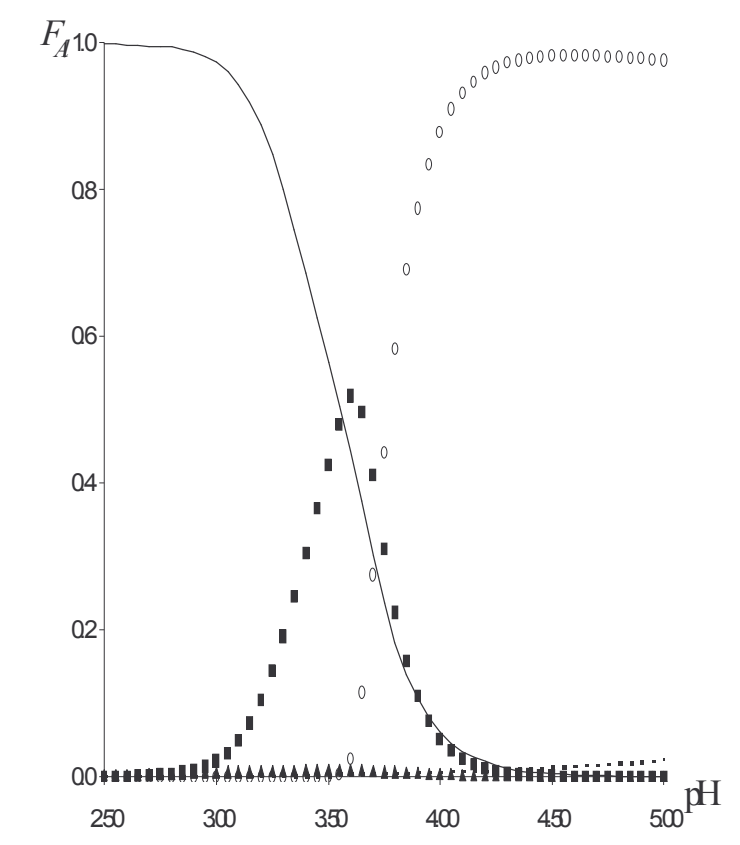

(a)

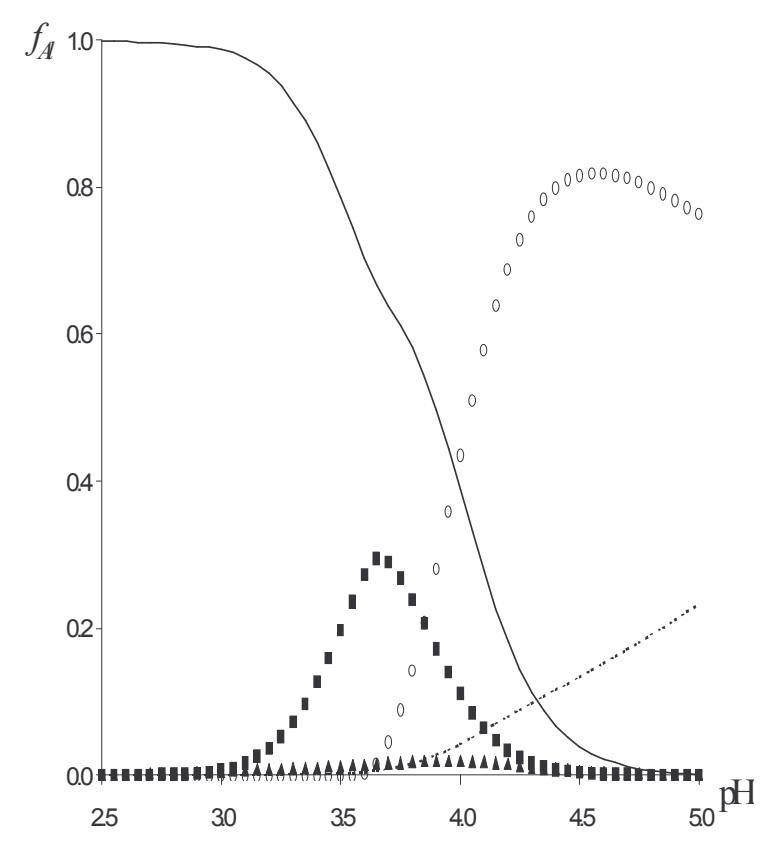

(b)

Figura 1.13. (a) Diagrama de fracciones molares de Componente para el sistema $\mathrm{Al}(\mathrm{III})-\mathrm{H}_{2} \mathrm{O}$; (b) Diagrama de fracciones molares de especies para el sistema $\mathrm{Al}(\mathrm{III})-\mathrm{H}_{2} \mathrm{O}$. (-) Fracción de $\mathrm{Al}^{3+},(\mathbf{\Delta})$ Fracción de $\mathrm{Al}(\mathrm{OH})_{2}{ }^{+}$(- - ) Fracción de $\mathrm{Al}(\mathrm{OH})_{3}$, (ם) Fracción de $\mathrm{Al}_{3}(\mathrm{OH})_{4}{ }^{5+}$ y $(\mathrm{O})$ Fracción de $\mathrm{Al}_{12}(\mathrm{OH})_{32}{ }^{7+}$ (Obtenidos a partir del modelo químico propuesto en la Tabla 2.7).

La Figura 1.13 muestra los diagramas de fracciones molares tanto de componente como de especie para el sistema $\mathrm{Al}(\mathrm{III})-\mathrm{H}_{2} \mathrm{O}$ obtenidos con el programa computacional MEDUSA, con estas fracciones se compararon las áreas obtenidas a partir de los espectros de RMN. En la Figura 1.14 se presenta la comparación entre las fracciones de área obtenidas experimentalmente de los espectros de $\mathrm{RMN} \mathrm{de}{ }^{27} \mathrm{Al}$ y las fracciones molares de componente (para ambos modelos propuestos), observándose que en ninguno de los casos estas se ajustan adecuadamente. Por otro lado, en la Figura 1.15 se muestra la comparación entre las fracciones de área experimentales y las fracciones de especie calculadas, observándose un adecuado ajuste de estas. Con la finalidad de lograr una mejor sobreposición se variaron mediante iteración las constantes de formación asociadas a cada 


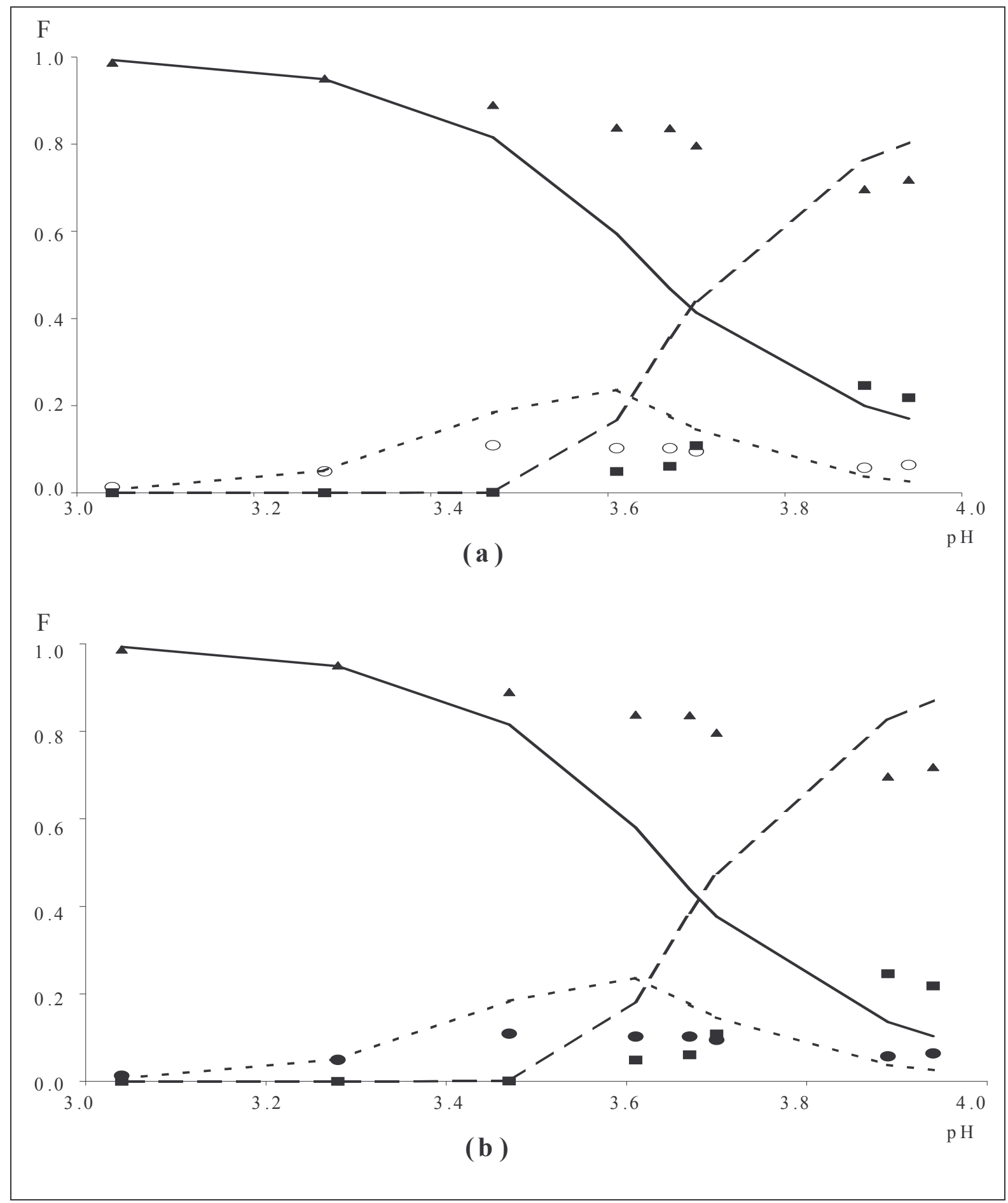

Figura 1.14. Comparación entre la fracciones de área obtenidas experimentalmente de los espectros de RMN de ${ }^{27} \mathrm{Al}$ y las fracciones molares de componente determinadas a partir del modelo de la Tabla 1.7. (ム) Fracción experimental de 0ppm, ( $\bigcirc)$ fracción experimental de 5ppm, ( $\mathbf{\square})$ fracción experimental de 63ppm (a) (- ) Fracción estimada de $\mathrm{Al}^{3+}, \mathrm{Al}(\mathrm{OH})^{2+}$ y $\mathrm{Al}(\mathrm{OH})_{3}$ y fracción proporcional de $\mathrm{Al}_{13}(\mathrm{OH})_{32}{ }^{7+}$ (- - ) Fracción estimada de $\mathrm{Al}_{3}(\mathrm{OH})_{4}{ }^{5+}$, (- -$)$ Fracción estimada proporcional de $\mathrm{Al}_{13}(\mathrm{OH})_{32}{ }^{7+}$; (b) (- ) Fracción estimada de $\mathrm{Al}^{3+}, \mathrm{Al}(\mathrm{OH})^{2+}$ y $\mathrm{Al}(\mathrm{OH})_{3}$; (- - ) Fracción estimada de $\mathrm{Al}_{3}(\mathrm{OH})_{4}{ }^{5+}$, (-) Fracción estimada de $\mathrm{Al}_{13}(\mathrm{OH})_{32}{ }^{7+}$ 


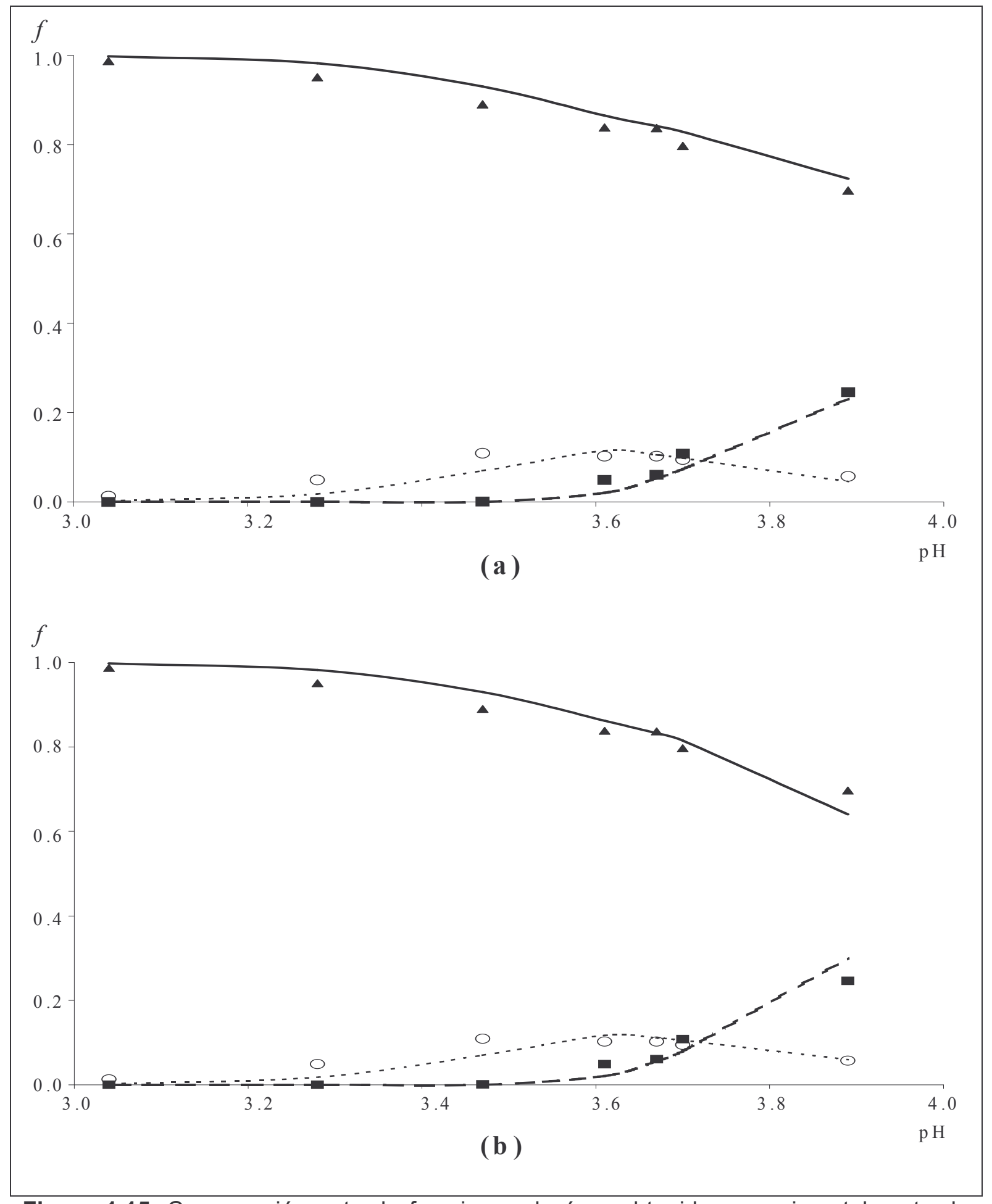

Figura 1.15. Comparación entre la fracciones de área obtenidas experimentalmente de los espectros de RMN de ${ }^{27} \mathrm{Al}$ y las fracciones molares de especie determinadas a partir del modelo de la Tabla 1.7. (ム) Fracción experimental de 0ppm, $(\bigcirc)$ fracción experimental de 5ppm, (ם) fracción experimental de 63ppm (a) (- ) Fracción estimada de $\mathrm{Al}^{3+}, \mathrm{Al}(\mathrm{OH})^{2+}$ y $\mathrm{Al}(\mathrm{OH})_{3}$ y fracción proporcional de $\mathrm{Al}_{13}(\mathrm{OH})_{32}{ }^{7+}$ (- - ) Fracción estimada de $\mathrm{Al}_{3}(\mathrm{OH})_{4}{ }^{5+}$, (- - Fracción estimada proporcional de $\mathrm{Al}_{13}(\mathrm{OH})_{32}{ }^{7+}$; (b) (- ) Fracción estimada de 
$\mathrm{Al}^{3+}, \mathrm{Al}(\mathrm{OH})^{2+}{ }_{7+} \mathrm{Al}(\mathrm{OH})_{3} ;$ (- - ) Fracción estimada de $\mathrm{Al}_{3}(\mathrm{OH})_{4}{ }^{5+},(--)$ Fracción estimada de $\mathrm{Al}_{13}(\mathrm{OH})_{32}{ }^{7+}$

especie, obteniéndose el modelo que se presenta en la Tabla 1.7, observándose que los valores estimados de las constantes para obtener un ajuste adecuado no son significativamente diferentes de las propuestas en la Tabla 1.5. Con lo anterior se establece que las fracciones molares de especie definen el comportamiento de las señales. Es importante destacar que independientemente del modelo empleado para la comparación de las fracciones de área con las fracciones de especie calculadas, el ajuste de las fracciones es similar, lo cual no permite establecer de manera contundente la naturaleza química de la señal que aparece en 63ppm. Con la aplicación del algoritmo propuesto se logra evidenciar que el modelo químico propuesto para el sistema $\mathrm{Al}(\mathrm{III})-\mathrm{H}_{2} \mathrm{O}$ es adecuado también para explicar el comportamiento observado en RMN. El hecho de obtener una relación entre las fracciones de área de las señales de RMN y las fracciones molares de especie es de gran importancia e impacto en lo que respecta a estudios de RMN, ya que corrobora lo sugerido en el estudio realizado para el sistema $\mathrm{B}(\mathrm{III})-\mathrm{H}_{2} \mathrm{O}$.

Solamente Bottero $y$ colaboradores ${ }^{7}$ habían propuesto con anterioridad una relación de las fracciones de componente y las áreas de las señales determinando una constante para la especie $\left.\mathrm{Al}_{13}(\mathrm{OH})_{32}\right)^{7+}$ de $-\log * \beta_{13,36}=-105.5$. Sin embargo, este resultado podría estar equivocado, debido a que estos autores observaron una señal en 80ppm en medio ácido la cual se adjudicó a una especie dimérica de Al(III) y que por tanto influye en el ajuste de las fracciones de área experimentales. No obstante esa señal no ha sido observada en los estudios previos realizados por diversos autores ${ }^{41-49}$. 
Tabla 1.7. Constantes de formación estimadas para el ajuste de las fracciones molares de especie con las fracciones de área experimentales determinadas de los espectros de RMN $\mathrm{de}^{27} \mathrm{Al}$

\section{Especies}

$$
-\log * \beta
$$

\begin{tabular}{cc}
$\mathrm{Al}(\mathrm{OH})^{2+}$ & 5.29 \\
$\mathrm{Al}(\mathrm{OH})_{3}$ & 12.06 \\
$\mathrm{Al}_{3}(\mathrm{OH})_{4}{ }^{5+}$ & 14.10 \\
$\mathrm{Al}_{13}(\mathrm{OH})_{32}{ }^{7+}$ & 110.60 \\
\hline
\end{tabular}

Además, Marklund y Öhman ${ }^{41}$ habían realizado con anterioridad estudios para relacionar las fracciones de área de señales de RMN con fracciones estimadas para cada especie. Desafortunadamente estos autores no proporcionan detalles sobre los espectros de $\mathrm{RMN}$ de ${ }^{27} \mathrm{Al}$, ni sobre la determinación de las fracciones de área experimentales o los algoritmos empleados, además de que el valor ajustado para el tridecámero fue de $\log * \beta_{13,32}=-105.5$, mientras que el obtenido por uno de estos mismos autores en estudios potenciométricos ${ }^{8}$ resultó de $\log * \beta_{13,32}=-109.2$, valores que puede observarse difieren de manera importante.

Por otro lado y con la finalidad de tener mayor información acerca de las señales observadas en los estudios de $\mathrm{RMN}$ de ${ }^{27} \mathrm{Al}$, se llevó a cabo un estudio mediante diluciones sucesivas de una solución de Al(III) $1.00 \mathrm{M}$ parcialmente hidrolizada $(\mathrm{OH} / \mathrm{Al}(\mathrm{III})=0.5)$, obteniéndose los espectros de $\mathrm{RMN}$ de ${ }^{27} \mathrm{Al}$ que se muestran en la Figura 1.16. En ella se puede observar que el pH de la solución se incrementa conforme la dilución es mayor. 
Además se puede ver que la señal en 0ppm sufre un ensanchamiento lo cual se explica por la formación de las especies monoméricas $\mathrm{Al}(\mathrm{OH})^{2+}$ y $\mathrm{Al}(\mathrm{OH})_{3}$ ya que ambas especies presentan al igual que el catión solvatado $\left(\mathrm{Al}^{3+}\right)$ una geometría octaédrica. En la misma Figura 1.16 se aprecia la señal en $5 \mathrm{ppm}$, que está relacionada con la especie $\mathrm{Al}_{3}(\mathrm{OH})_{4}{ }^{5+}$. Dicha señal desaparece conforme aumenta la dilución, lo cual era de esperarse por tratarse de una especie polinuclear. Además, es posible ver que la señal en $63 \mathrm{ppm}$, relacionada con la especie $\mathrm{Al}_{13}(\mathrm{OH})_{32}{ }^{7+}$, es una señal muy débil. Es importante destacar que esta aparece al incrementar la dilución, lo que podría resultar extraño, considerando que a mayores concentraciones debería de favorecerse la formación de la especie polinuclear con la que esta señal se asocia. Sin embargo, hay que notar que el pH del sistema también aumenta favoreciendo la formación de esta especie. Otro punto importante de analizar es el hecho de que la altura o el área de la señal en 63ppm no mantiene una relación 1:12 como lo mencionan Akkit y Elders ${ }^{45}$ en un estudio similar realizado, lo cual no da validez a la idea de estos autores que establece que la señal de $63 \mathrm{ppm}$ corresponde solamente a un sólo núcleo de aluminio con geometría tetraédrica.

Con la finalidad de validar el modelo potenciométrico propuesto en la Tabla 1.5 se llevó a cabo un ajuste de las fracciones molares de especie (calculadas con el programa computacional MEDUSA) y las fracciones de área experimentales. En la Tabla 1.8 se muestran los resultados del ajuste de dichas fracciones al probar dos diferentes modelos, mientras que en la Tabla 1.9 se presenta el error estimado, como la raíz media cuadrática de los residuales (rmc) obtenidos de la diferencia entre las fracciones de área experimentales 


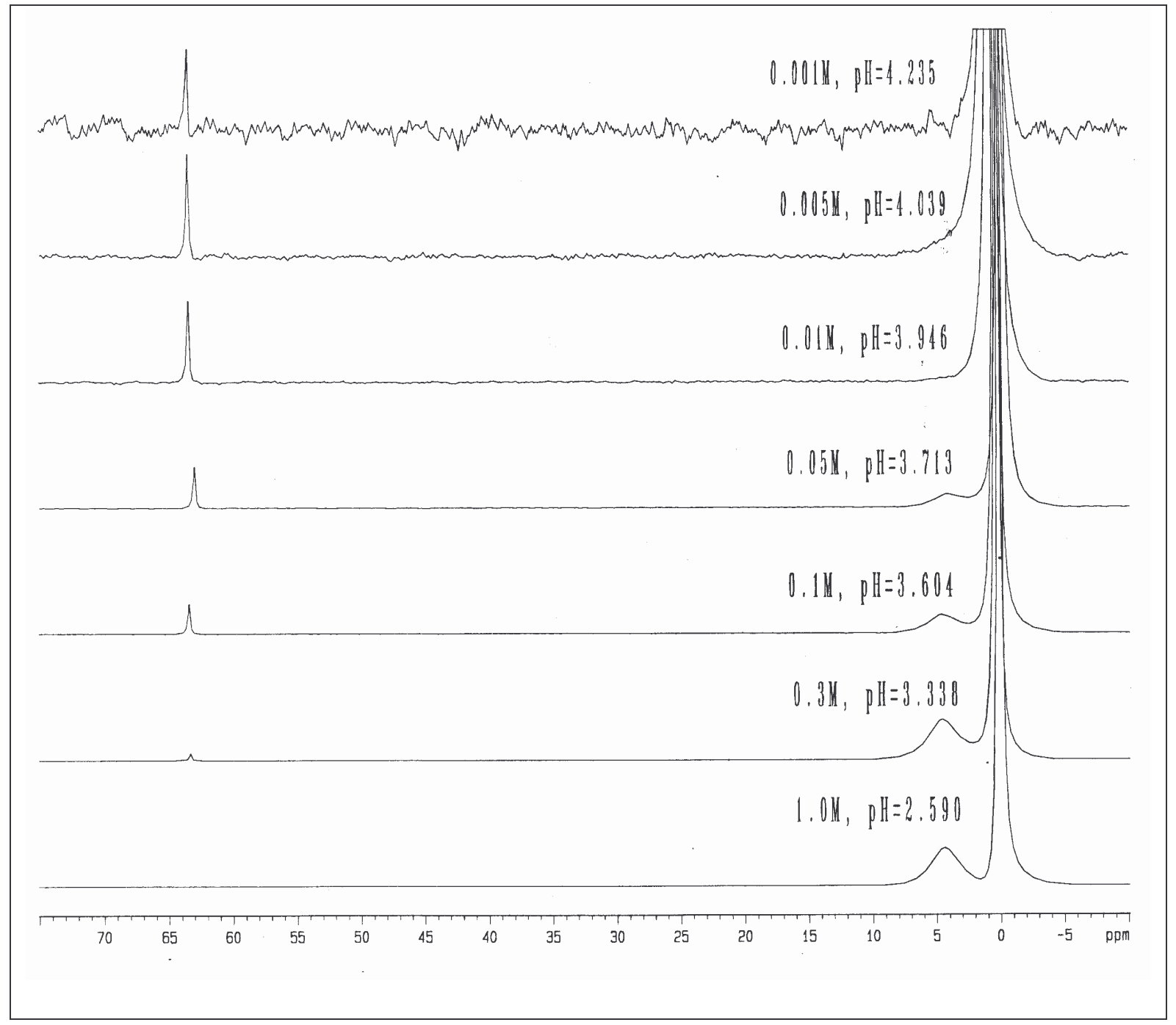

Figura 1.16. Espectros de RMN para ${ }^{27} \mathrm{Al}$ para soluciones diluidas sucesivamente a partir de un sistema con $[\mathrm{Al}(\mathrm{III})]_{\text {total }}=1.000 \mathrm{M}$. y $\mathrm{OH} / \mathrm{Al}(\mathrm{III})=0.5$

y las fracciones calculadas. Los datos en el ajuste del modelo que considera solamente a las especies $\mathrm{Al}(\mathrm{OH})^{2+} \mathrm{y}_{3}(\mathrm{OH})_{4}{ }^{5+}$ (modelo 1) es considerablemente menor que el del modelo que toma en cuenta tanto a dichas especies como a $\mathrm{Al}(\mathrm{OH})_{3}$ y $\mathrm{Al}_{13}(\mathrm{OH})_{32}{ }^{7+}$, además de no presentar tendencia. Por lo tanto se concluye que en el estudio de diluciones sólo se tiene información suficiente para ajustar las constantes de equilibrio de las especies $\mathrm{Al}(\mathrm{OH})^{2+} \mathrm{y}$ $\mathrm{Al}_{3}(\mathrm{OH})_{4}{ }^{5+}$. Con base en lo anterior es posible determinar que los valores obtenidos para las especies $\mathrm{Al}(\mathrm{OH})^{2+}$ y $\mathrm{Al}_{3}(\mathrm{OH})_{4}{ }^{5+}$ son muy similares a los calculados en estudios tanto 
potenciométricos como de RMN, lo cual permite validar el modelo propuesto en la Tabla 1.5.

Tabla 1.8. Constantes de formación estimadas por $\mathrm{RMN}$ de ${ }^{27} \mathrm{Al}$ a partir del experimento de diluciones sucesivas

\begin{tabular}{ccc}
\hline Especies & Modelo 1 & Modelo 2 \\
& $-\log * \boldsymbol{\beta}$ & $-\log * \boldsymbol{\beta}$ \\
\hline $\mathrm{Al}(\mathrm{OH})^{2+}$ & $5.10-5.60$ & $5.29-5.90$ \\
$\mathrm{Al}(\mathrm{OH})_{3}$ & No considerada & $12.40-14.00$ \\
$\mathrm{Al}_{3}(\mathrm{OH})_{4}{ }^{5+}$ & $12.91-13.80$ & $12.90-13.70$ \\
$\mathrm{Al}_{13}(\mathrm{OH})_{32}{ }^{7+}$ & No considerada & $103.70-106.00$ \\
\hline
\end{tabular}

Tabla 1.9. Raíz media cuadrática de los residuales de las fracciones obtenidas con los modelos propuestos y las fracciones de área experimentales para el experimento de diluciones sucesivas

\begin{tabular}{|c|c|c|}
\hline Señal de $\mathrm{RMN}$ de ${ }^{27} \mathrm{Al}$ & $\begin{array}{l}\text { Modelo } 1 \\
\operatorname{rmc}\left(10^{5}\right)\end{array}$ & $\begin{array}{l}\text { Modelo } 2 \\
\text { rmc }\left(10^{5}\right)\end{array}$ \\
\hline 0 ppm (monómeros) & 6.8 & 307.5 \\
\hline 5 ppm (trímero) & 6.8 & 45.8 \\
\hline 63 ppm (tridecámero) & 0.00 & 145.4 \\
\hline Total rms & 4.8 & 216.5 \\
\hline
\end{tabular}




\subsubsection{CONCLUSIONES}

Con base en los resultados obtenidos en este trabajo se puede establecer el modelo propuesto para el sistema $\mathrm{Al}(\mathrm{III})-\mathrm{H}_{2} \mathrm{O}$ en el que se propone la formación de dos especies mononucleares $\left(\mathrm{Al}(\mathrm{OH})_{2}{ }^{+}\right.$y $\left.\mathrm{Al}(\mathrm{OH})_{3}\right)$, así como la de un oligómero de estequiometría $(3,4)$ $\left(\mathrm{Al}_{3}(\mathrm{OH})_{4}^{5+}\right)$ y la de una especie polinuclear de alto peso molecular con una relación de aluminio:hidróxido de $2.46\left(\mathrm{Al}_{13}(\mathrm{OH})_{32}{ }^{7+}\right)$. Este modelo se considera adecuado, ya que éste permite explicar no sólo la información potenciométrica sino también los estudios de conductimetría realizados, así como el comportamiento observado experimentalmente en los espectros de RMN para ${ }^{27} \mathrm{Al}$. Además es un modelo químico en el que se esta considerando para su corroboración la geometría y estructura de las especies químicas propuestas.

Al igual que para el sistema $\mathrm{B}(\mathrm{III})-\mathrm{H}_{2} \mathrm{O}$ fue posible llevar a cabo un estudio cuantitativo de las señales de RMN observadas que llevó a establecer que las señales debidas a ${ }^{27} \mathrm{Al}$ son directamente proporcionales a las fracciones molares de especie, lo cual corrobora el algoritmo propuesto en este trabajo para analizar cuantitativamente las señales de resonancia magnética nuclear. 


\subsection{SISTEMA Ga(III)- $\mathrm{H}_{2} \mathrm{O}$}

\subsubsection{ANTECEDENTES}

La hidrólisis de galio(III) en solución acuosa se ha estudiado menos exhaustivamente que la de aluminio(III), sin embargo los pocos estudios que se tienen sobre la determinación de las especies y sus respectivas constantes coinciden en su mayoría en la posible formación de especies mononucleares únicamente. Solamente Baes y Mesmer ${ }^{1}$ establecen la posible formación de la especie polinuclear $\mathrm{Ga}_{26}(\mathrm{OH})_{65}{ }^{13+}$ la cual proponen podría formarse en soluciones sobresaturadas. Sin embargo hacen mención de la dificultad que podría representar el evidenciarla y mantener como una especie estable debido a la precipitación de $\mathrm{GaO}(\mathrm{OH})$.

Pese a que la mayoría de los estudios apuntan hacia la formación de especies mononucleares en solución, no hay concordancia total entre los diferentes autores. Se llegan a tener modelos químicos que consisten en la formación únicamente de la especie de estequiometría $(1,1)$ hasta modelos que sugieren la formación de especies que van desde la $(1,1)$ hasta la $(1,4)$. Por otro lado, tampoco se tiene una consistencia en los valores reportados para las constantes de formación de las diferentes especies 
Tabla 1.10. Modelos de constantes de formación propuestos para la hidrólisis de Ga(III)

\begin{tabular}{|c|c|c|c|}
\hline AUTOR(ES) & $\begin{array}{l}\text { Especie } \\
\text { Química }\end{array}$ & $-\log \beta^{*}$ & Método \\
\hline Wilson A.S. y Taube H. ${ }^{51}$ & $\mathrm{Ga}(\mathrm{OH})^{2+}$ & 2.92 & Espectroscopía \\
\hline $\begin{array}{l}\text { Nazarenko V.A. y Antonovich } \\
\text { V.P. }{ }^{52}\end{array}$ & $\begin{array}{c}\mathrm{Ga}(\mathrm{OH})^{2+} \\
\mathrm{Ga}(\mathrm{OH})_{2}{ }^{+} \\
\mathrm{Ga}(\mathrm{OH})_{3}\end{array}$ & $\begin{array}{c}2.91 \\
6.61 \\
11.02\end{array}$ & Potenciometría \\
\hline Baes C.F. y Mesmer R.E & $\begin{array}{c}\mathrm{Ga}(\mathrm{OH})^{2+} \\
\mathrm{Ga}(\mathrm{OH})_{2}{ }^{+} \\
\mathrm{Ga}(\mathrm{OH})_{3} \\
\mathrm{Ga}(\mathrm{OH})_{4}^{-}\end{array}$ & $\begin{array}{c}2.6 \\
5.9 \\
10.3 \\
16.6\end{array}$ & Potenciometría \\
\hline Yamada S, et al. ${ }^{53}$ & $\mathrm{Ga}(\mathrm{OH})^{2+}$ & $2.89(0.03)$ & $\begin{array}{l}\text { Espectroscopía y } \\
\text { Potenciometría }\end{array}$ \\
\hline Tóth I., Zékány L. y Brücher E. ${ }^{37}$ & $\begin{array}{c}\mathrm{Ga}(\mathrm{OH})^{2+} \\
\mathrm{Ga}(\mathrm{OH})_{2}{ }^{+} \\
\mathrm{Ga}(\mathrm{OH})_{3} \\
\mathrm{Ga}(\mathrm{OH})_{4}^{-}\end{array}$ & $\begin{array}{c}2.41 \\
5.92 \\
10.63 \\
16.87\end{array}$ & Potenciometría \\
\hline Campisi A. y Tregloan P. ${ }^{54}$ & $\begin{array}{l}\mathrm{Ga}(\mathrm{OH})^{2+} \\
\mathrm{Ga}(\mathrm{OH})_{2}^{+}\end{array}$ & $\begin{array}{l}4.17 \\
8.21\end{array}$ & Potenciometría \\
\hline Brown P. ${ }^{55}$ & $\begin{array}{l}\mathrm{Ga}(\mathrm{OH})^{2+} \\
\mathrm{Ga}(\mathrm{OH})_{2}^{+}\end{array}$ & $\begin{array}{l}3.16(4.89 \%) \\
7.07(5.58 \%)\end{array}$ & Potenciometría \\
\hline Borgias B., Hugi A., Raymond K. ${ }^{56}$ & $\begin{array}{l}\mathrm{Ga}(\mathrm{OH})_{3} \\
\mathrm{Ga}(\mathrm{OH})_{4}^{-}\end{array}$ & $\begin{array}{l}11.63 \\
17.5\end{array}$ & Potenciometría \\
\hline
\end{tabular}

En la Tabla 1.10 se muestran los modelos reportados para la hidrólisis de galio(III). En ella se puede observar que no sólo no hay consistencia en los modelos, sino también 
que existe una diferencia marcada en algunos de los valores de las constantes, además es importante destacar que sólo dos de los modelos reportados dan la desviación estándar sobre los valores de las constantes, lo cual implica el conocimiento de la estadística sobre el modelo, con lo que de alguna forma se le da certidumbre a este.

Al igual que para aluminio(III), este grupo de investigación en un trabajo anterior ${ }^{48}$ realizó estudios tanto de potenciometría como de RMN para poder identificar las especies que se forman durante el proceso de hidrólisis, así como sus constantes respectivas. Se

determinó que sólo se forman las especies monoméricas $\mathrm{Ga}(\mathrm{OH})_{2}{ }^{+2}$ y $\mathrm{Ga}(\mathrm{OH})_{3}$ con constantes de formación de 7.07(0.03) y $10.71(0.01)$ respectivamente. En este trabajo se busca dar un mayor soporte al modelo previamente reportado realizando para ello estudios más detallados de potenciometría, así como estudios de conductimetría, con el propósito de poder reportar un modelo confiable para el sistema $\mathrm{Ga}(\mathrm{III})-\mathrm{H}_{2} \mathrm{O}$.

\subsubsection{EXPERIMENTAL}

\subsubsection{Estudios Potenciométricos}

Se prepararon soluciones acuosas de $\mathrm{Ga}(\mathrm{III})$ partiendo de $\mathrm{Ga}\left(\mathrm{NO}_{3}\right)_{3} \bullet 9 \mathrm{H}_{2} \mathrm{O}$ (Baker Analyzed, qp) en un intervalo de $3.47 \times 10^{-4} \mathrm{M}$ a $17.36 \times 10^{-4} \mathrm{M}$. Al menos dos muestras de cada solución se valoraron con soluciones estandarizadas de hidróxido de sodio, preparadas a partir del procedimiento propuesto en la sección como se indicó en la sección 1.1.2.1. En todos los casos se registraron las variaciones de $\mathrm{pH}$ (al menos 60 datos) con un pH-metro Radiometer Tacussel LPH430T ( $\Delta \mathrm{pH} \pm 0.001)$, empleando un electrodo de vidrio 
combinado con referencia interna de $\mathrm{Ag} / \mathrm{AgCl}_{(\mathrm{s})}$; efectuando la calibración del equipo con sistemas buffer de referencia de $\mathrm{pH}=4.005$. En todos los experimentos se controló la temperatura con un baño (VWR, modelo 12101-10, y un flujo de nitrógeno se mantuvo constante durante las valoraciones En la preparación de todas las soluciones se empleo agua desionizada y descarbonatada de Tipo I (desionizador Millipore). La concentración analítica de $\mathrm{Ga}(\mathrm{III})$ se determinó complejométricamente ${ }^{57}$.

\subsubsection{Estudios Conductimétricos}

Se realizaron valoraciones conductimétricas de soluciones acuosas de Ga(III) $\left(\mathrm{Ga}\left(\mathrm{NO}_{3}\right)_{3} \cdot 9 \mathrm{H}_{2} \mathrm{O}\right.$, Baker Analyzed, qp) en un intervalo de concentraciones de $3.20 \mathrm{X} 10^{-4}$ M a $16.00 \times 10^{-4} \mathrm{M}$, valorando con soluciones estandarizadas de hidróxido de sodio. El registro de la conductividad se efectuó con ayuda de un conductimetro Radiometer Copenhagen CDM230 empleando una celda conductimétrica de cuatro polos (con cuatro anillos de platino) Radiometer Copenhagen CDC865. Se realizó la calibración del equipo antes de cada valoración con soluciones estándar de $\mathrm{NaCl}$.

La temperatura se mantuvo constante a $25.0{ }^{\circ} \mathrm{C}\left(\Delta \mathrm{T} \pm 0.1{ }^{\circ} \mathrm{C}\right)$ por medio de un baño de agua (VWR PolyScience modelo 12101) y empleándose celdas de vidrio de doble camisa. Además se mantuvo un flujo constante de nitrógeno de alta pureza para evitar la carbonatación del sistema. El agua empleada en todos los experimentos y en la preparación de la soluciones fue agua desionizada y descarbonatada Tipo 1 (Millipore). 


\subsubsection{RESULTADOS Y ANÁLISIS}

\subsubsection{Estudios Potenciométricos}

En la Figura 1.17 se muestran las curvas características de valoración para las soluciones acuosas de Ga(III). En ella se puede observar una disminución del pH conforme la concentración total de $\mathrm{Ga}(\mathrm{III})$ aumenta, además se aprecia en todos los casos un incremento del $\mathrm{pH}$ hasta una relación $\mathrm{OH} / M=2.5$. Por encima de este valor tiene lugar la precipitación, por lo que la valoración sólo se desarrolló hasta la relación antes mencionada.

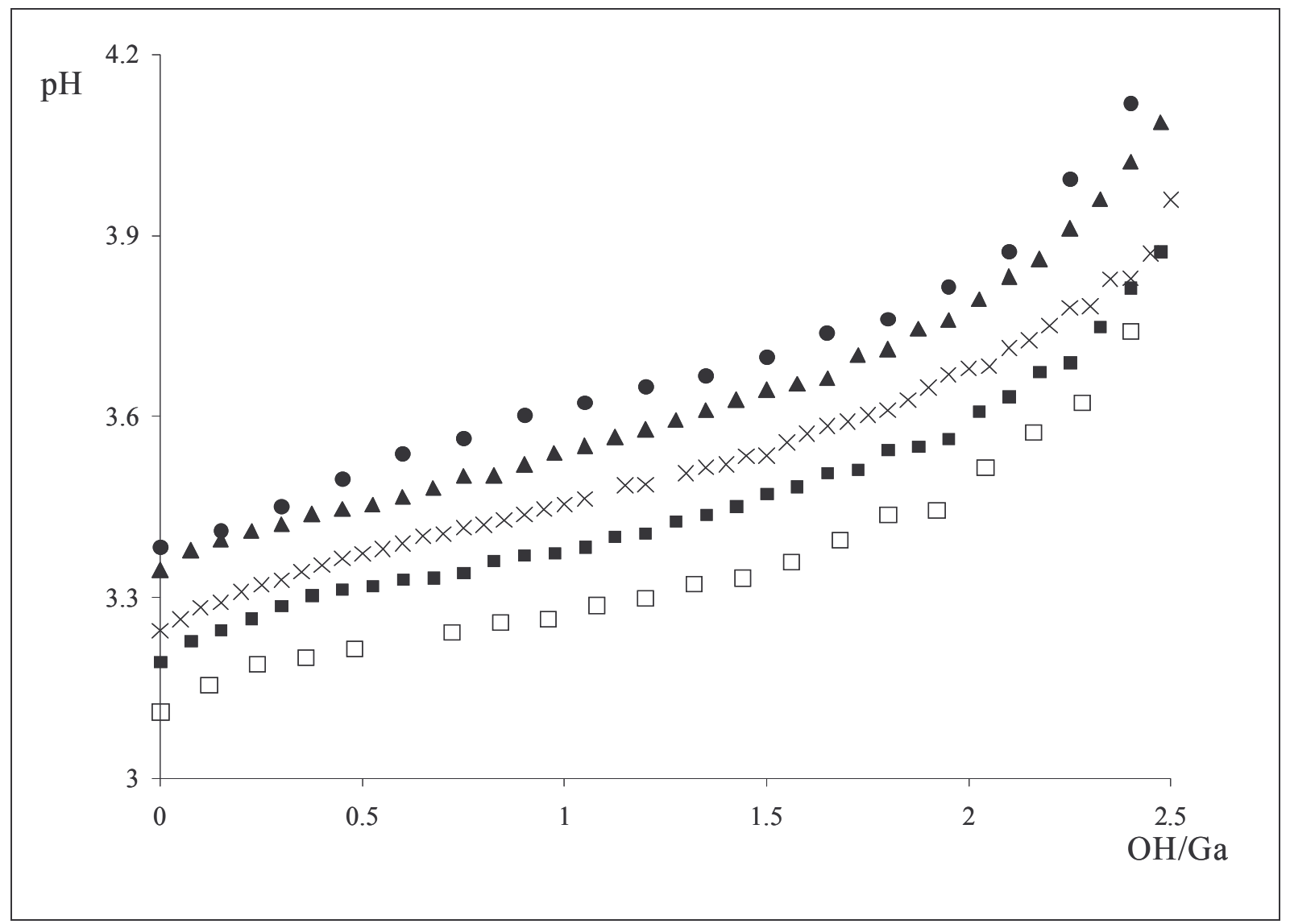

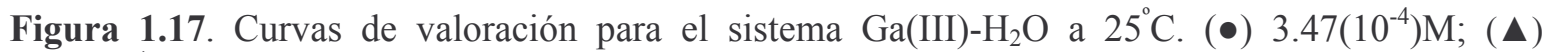
6.94 $\left(10^{-4}\right) \mathrm{M} ;(\mathrm{X}) 10.42\left(10^{-4}\right) \mathrm{M},(\square) 13.89\left(10^{-4}\right) \mathrm{M},(\square) 17.36\left(10^{-4}\right) \mathrm{M}$.

Los datos obtenidos de las valoraciones potenciométricas fueron alimentados al 
programa SUPERQUAD para el refinamiento de las constantes de equilibrio. Para ello se tomó en consideración tanto a especies monoméricas y poliméricas de acuerdo al equilibrio de la ecuación 1.11. Los resultados relacionados con el mejor refinamiento obtenido por SUPERQUAD en este trabajo se muestran en la Tabla 1.11. En ella se puede observar que solamente se pudieron determinar especies monoméricas, lo cual concuerda con los estudios realizados por otros autores ${ }^{37,51-56}$. Incluso Brown y cols. ${ }^{55}$, en estudios realizados en un intervalo de $0.10 \mathrm{M} \leq[\mathrm{Ga}(\mathrm{III})] \leq 1.5 \mathrm{M}$, no reportan la formación de especies polinucleares. Además, es importante señalar, que el modelo determinado en este trabajo es similar al modelo propuesto en un trabajo anterior realizado por este mismo grupo de $\operatorname{trabajo}^{48}$. Todo lo anterior hace pensar y concluir que no se llegan a formar especies polinucleares durante el proceso de hidrólisis de $\mathrm{Ga}(\mathrm{III})$.

$$
\begin{array}{r}
\mathrm{iGa}^{3+}+\mathrm{jH}_{2} \mathrm{O}=\mathrm{Ga}_{\mathrm{i}}(\mathrm{OH})_{\mathrm{j}}^{3 \mathrm{i}-\mathrm{j}}+\mathrm{jH}^{+} \\
* \beta_{\mathrm{ij}}=\frac{\left[\mathrm{Ga}_{\mathrm{i}}(\mathrm{OH})_{\mathrm{k}}^{3 \mathrm{i}-\mathrm{k}}\right]\left[\mathrm{H}^{+}\right]^{\mathrm{j}}}{\left[\mathrm{Ga}^{3+}\right]^{\mathrm{i}}}
\end{array}
$$

Tabla 1.11. Valores de las constantes de formación para el mejor refinamiento determinado por el programa SUPERQUAD a partir de información potenciométrica del sistema $\mathrm{Ga}(\mathrm{III})-\mathrm{H}_{2} \mathrm{O}\left(\mathrm{T}=25^{\circ} \mathrm{C}\right)$

\begin{tabular}{ccc}
\hline Especies & $-\log { }^{*} \beta$ & Parámetros Estadísticos \\
\hline $\mathrm{Ga}(\mathrm{OH})_{2}{ }^{+}$ & $7.13 \pm 0.03$ & $\sigma^{\mathrm{b}}=0.5506$ \\
$\mathrm{Ga}(\mathrm{OH})_{3}$ & $10.62 \pm 0.01$ & $\mathrm{U}^{\mathrm{c}}=92.47$ \\
& $\left(\chi^{2}\right)^{\mathrm{d}}=62.75$ \\
\hline $\begin{array}{l}{ }^{\mathrm{a}} 350 \text { datos se alimentaron a SUPERQUAD. } \\
\text { residuales al cuadrados del potencial de celda. }{ }^{\mathrm{d}}{ }^{\mathrm{d}} \text { Chi-cuadrada para el refinamiento }\end{array}$
\end{tabular}


A partir de los datos potenciométricos se trazaron las curvas de la función de formación (Z) (Apéndice 1) como función del pH (Figura 1.18) de acuerdo a la ecuación 1.12 (que ha sido descrita ampliamente por Baes y Mesmer ${ }^{1}$ ) así como por Hartley et al. $^{58}$. Se puede observar que no existe una dependencia de $\mathrm{Z}$ en función a la concentración de $[\mathrm{Ga}(\mathrm{III})]_{\text {total }}$ lo cual es característico de sistemas en los que solamente existen especies mononucleares ${ }^{7}$, esto apoya los resultados obtenidos con el programa SUPERQUAD.

$$
Z=\left[\frac{\left[O H^{-}\right]_{\text {otal }}-\left[K_{w} / 10^{-p H}\right]+\left[10^{-p H}\right]}{[G a(I I I)]_{\text {total }}}\right]
$$

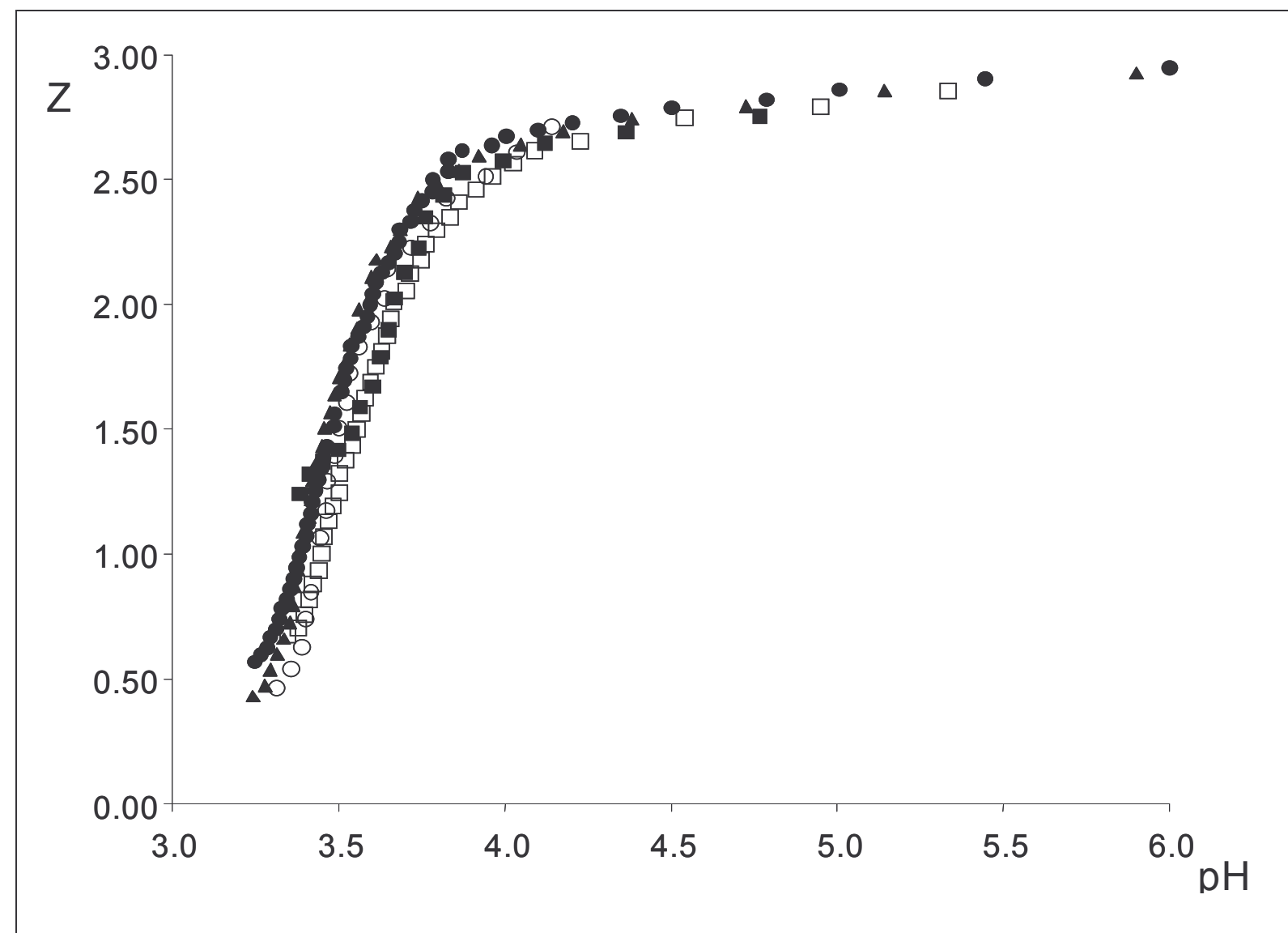

Figura 1.18. Gráfico de $\mathrm{Z}$ como función del $\mathrm{pH}$ para el sistema de $\mathrm{Ga}(\mathrm{III})-\mathrm{H}_{2} \mathrm{O}$, para diferentes concentraciones de $[\mathrm{Ga}(\mathrm{III})]_{\mathrm{t}}(\boldsymbol{\bullet}) 3.47\left(10^{-4}\right) \mathrm{M}$; (口) 6.94 $\left(10^{-4}\right) \mathrm{M} ;(\bullet) 10.42\left(10^{-4}\right) \mathrm{M} ;(\boldsymbol{\Delta}) 13.89\left(10^{-}\right.$ $\left.{ }^{4}\right) \mathrm{M},(\mathrm{O}) 17.36\left(10^{-4}\right) \mathrm{M}$. 
Las funciones de formación se ajustaron mediante la comparación gráfica de las funciones $\mathrm{Z}$ obtenidas experimentalmente y las determinadas a partir de la ecuación 1.13, en donde las fracciones molares de componente $F_{\mathrm{ij}}$ se determinaron con ayuda del programa computacional MEDUSA (Figura 1.19). En la Tabla 1.12 se muestran los valores de las constantes de equilibrio empleadas para obtener el ajuste gráfico que se muestra en la Figura 1.20. Es importante hacer mención que los valores obtenidos mediante este procedimiento gráfico son similares a los determinados por el programa computacional SUPERQUAD, lo cual valida en cierta medida el modelo químico para el sistema Ga(III)$\mathrm{H}_{2} \mathrm{O}$ propuesto en la Tabla 1.11.

$$
Z=\sum_{i}\left(\sum_{j}\left\{\left(\frac{j}{i}\right) F_{i j}\right\}\right)
$$

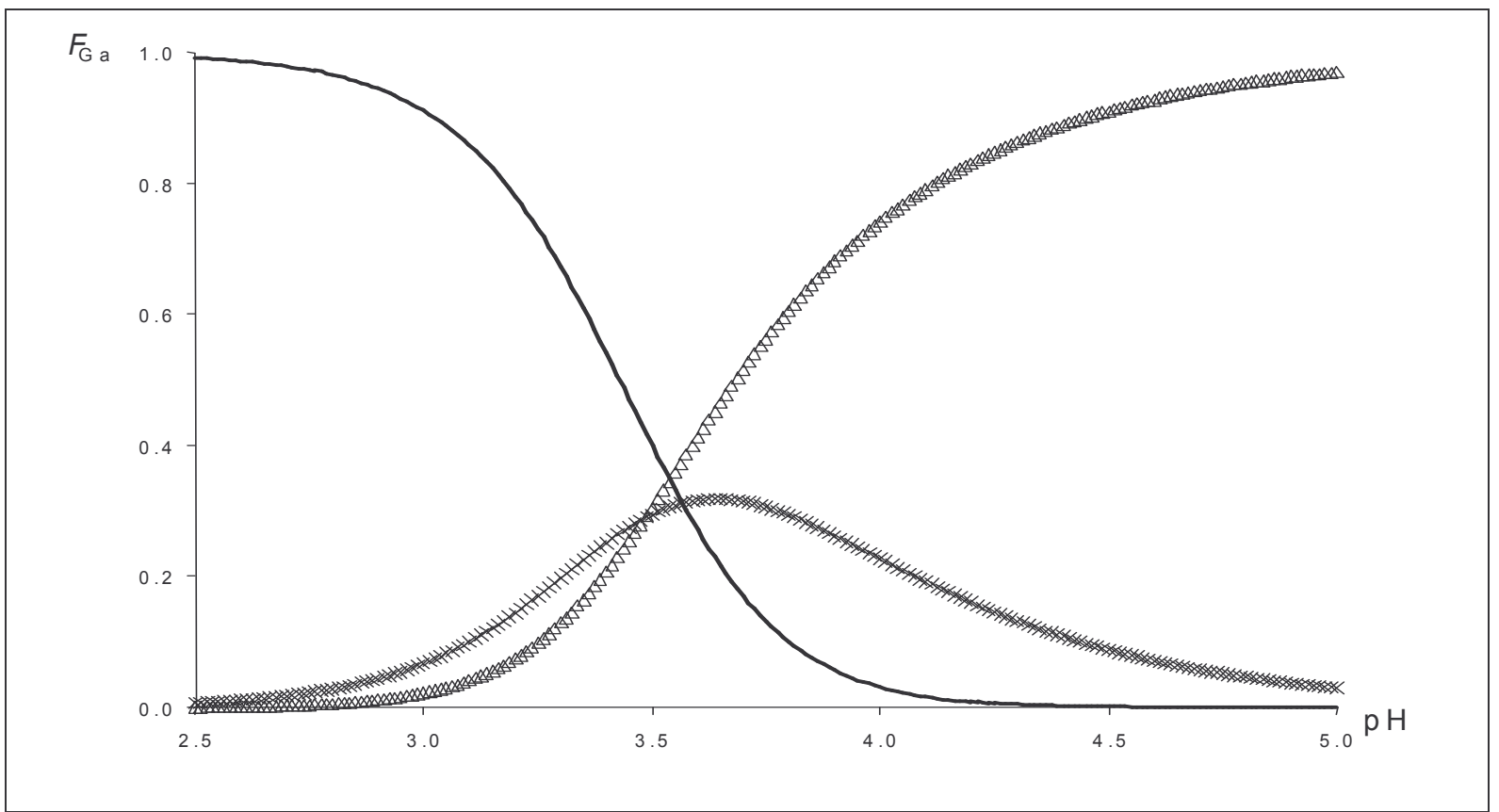

Figura 1.19. Diagrama de Distribución de Fracciones molares de componente para especies del sistema $\mathrm{Ga}(\mathrm{III})-\mathrm{H}_{2} \mathrm{O}$. (一) $\mathrm{Ga}^{3+},(\mathrm{X}) \mathrm{Ga}(\mathrm{OH})_{2}{ }^{+},(\Delta) \mathrm{Ga}(\mathrm{OH})_{3}$ 
Tabla 1.12. Constantes de formación para el sistema $\mathrm{Ga}(\mathrm{III})-\mathrm{H}_{2} \mathrm{O}$ determinadas por ajuste gráfico de la función $\mathrm{Z}$ con ayuda de MEDUSA ${ }^{\mathrm{a}}$.

\begin{tabular}{cc}
\hline Especie & $\operatorname{-og}^{*} \boldsymbol{\beta}$ \\
\hline $\mathrm{Ga}(\mathrm{OH})_{2}{ }^{+}$ & $6.85-7.50$ \\
$\mathrm{Ga}(\mathrm{OH})_{3}$ & $10.10-10.75$
\end{tabular}

El intervalo de concentraciones estudiado fue de $3.47\left(10^{-4}\right) \mathrm{M} \leq[\mathrm{Ga}(\mathrm{III})] \leq 17.36\left(10^{-4}\right) \mathrm{M}$

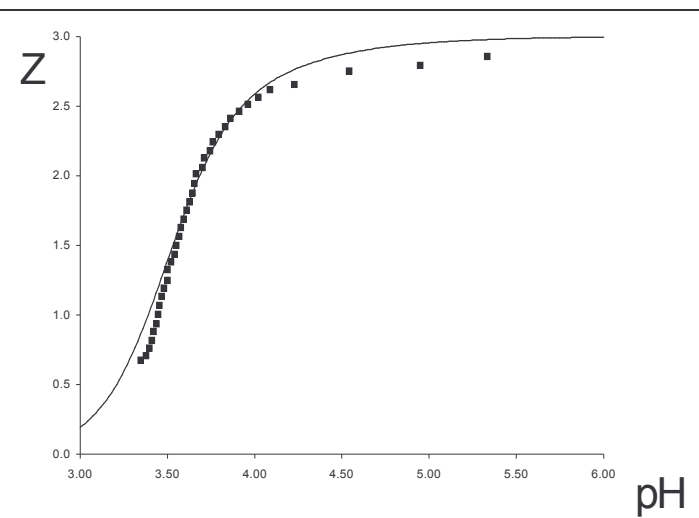

(a)

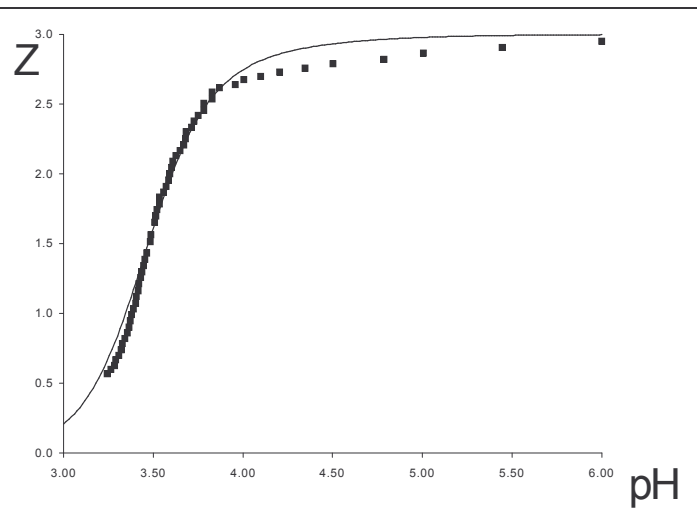

(b)

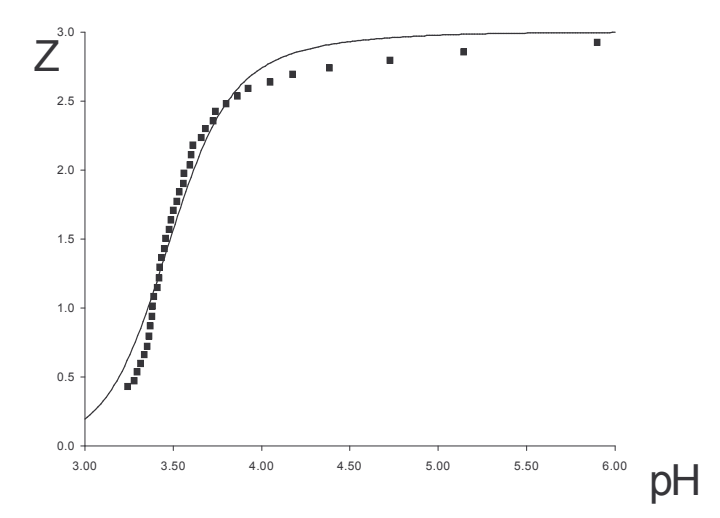

(c)

Figura 1.20. Gráfico de $\mathrm{Z}$ como función del $\mathrm{pH}$ para el sistema de $\mathrm{Ga}(\mathrm{III})-\mathrm{H}_{2} \mathrm{O}$, para diferentes concentraciones de $[\mathrm{Ga}(\mathrm{III})]_{\mathrm{t}}$. Las líneas continuas representan las simulaciones de $\mathrm{Z}$ determinadas a partir del modelo obtenido con SUPERQUAD para este sistema, mientras que los marcadores $(+)$ representan las $\mathrm{Z}$ experimentales. (a) $[\mathrm{Ga}(\mathrm{III})]_{\mathrm{T}}=6.94\left(10^{-4}\right) \mathrm{M}$; (b) $[\mathrm{Ga}(\mathrm{III})]_{\mathrm{T}}=10.42\left(10^{-4}\right) \mathrm{M}$; (c) $[\mathrm{Ga}(\mathrm{III})]_{\mathrm{T}}=13.89\left(10^{-4}\right) \mathrm{M}$ 


\subsubsection{2.. Estudios Conductimétricos}

Se efectuaron valoraciones conductimétricas de soluciones acuosas de Ga(III), las cuales se desarrollaron hasta antes de alcanzar una relación $\mathrm{OH} / \mathrm{Ga}(\mathrm{III})=2.5$ ya que después de esta tiene lugar la precipitación. En la Figura 1.21 se muestran las curvas obtenidas, observándose que en un intervalo de $3.2\left(10^{-4}\right) \mathrm{M} \leq[\mathrm{Ga}(\mathrm{III})] \leq 16.0\left(10^{-4}\right) \mathrm{M}$ las curvas presentan un comportamiento muy similar teniéndose una disminución en la conductividad conforme se va realizando la hidrólisis lo cual se debe a que la especie predominante es $\mathrm{Ga}(\mathrm{OH})_{3}$, la cual al no poseer carga no tiene una aportación a la conductividad del sistema. Para una concentración de $\mathrm{Ga}(\mathrm{III})$ 0.01M se observa por el contrario un pequeño incremento de la conductividad.

Se realizó el ajuste gráfico de las conductividades obtenidas experimentalmente y las estimadas con base en el modelo químico calculado por SUPERQUAD (Tabla 1.11) las cuales fueron determinadas mediante la ecuación 1.14. Las fracciones molares de las especies de $\mathrm{Ga}(\mathrm{III})$ se calcularon con ayuda del programa MEDUSA, la concentración de $\mathrm{Na}^{+}$se calculó con base en la cantidad de hidróxido de sodio agregada, la concentración de $\mathrm{NO}_{3}{ }^{-}$se estimó en relación a la cantidad inicial de $\mathrm{Ga}\left(\mathrm{NO}_{3}\right)_{3}$ y las concentraciones de $\mathrm{H}^{+}$y $\mathrm{OH}^{-}$se determinaron a partir del $\mathrm{pH}$ experimental. Los coeficientes de las conductividades equivalentes para cada especie $(\lambda)$ se estimaron mediante iteración (Tabla 1.13) 


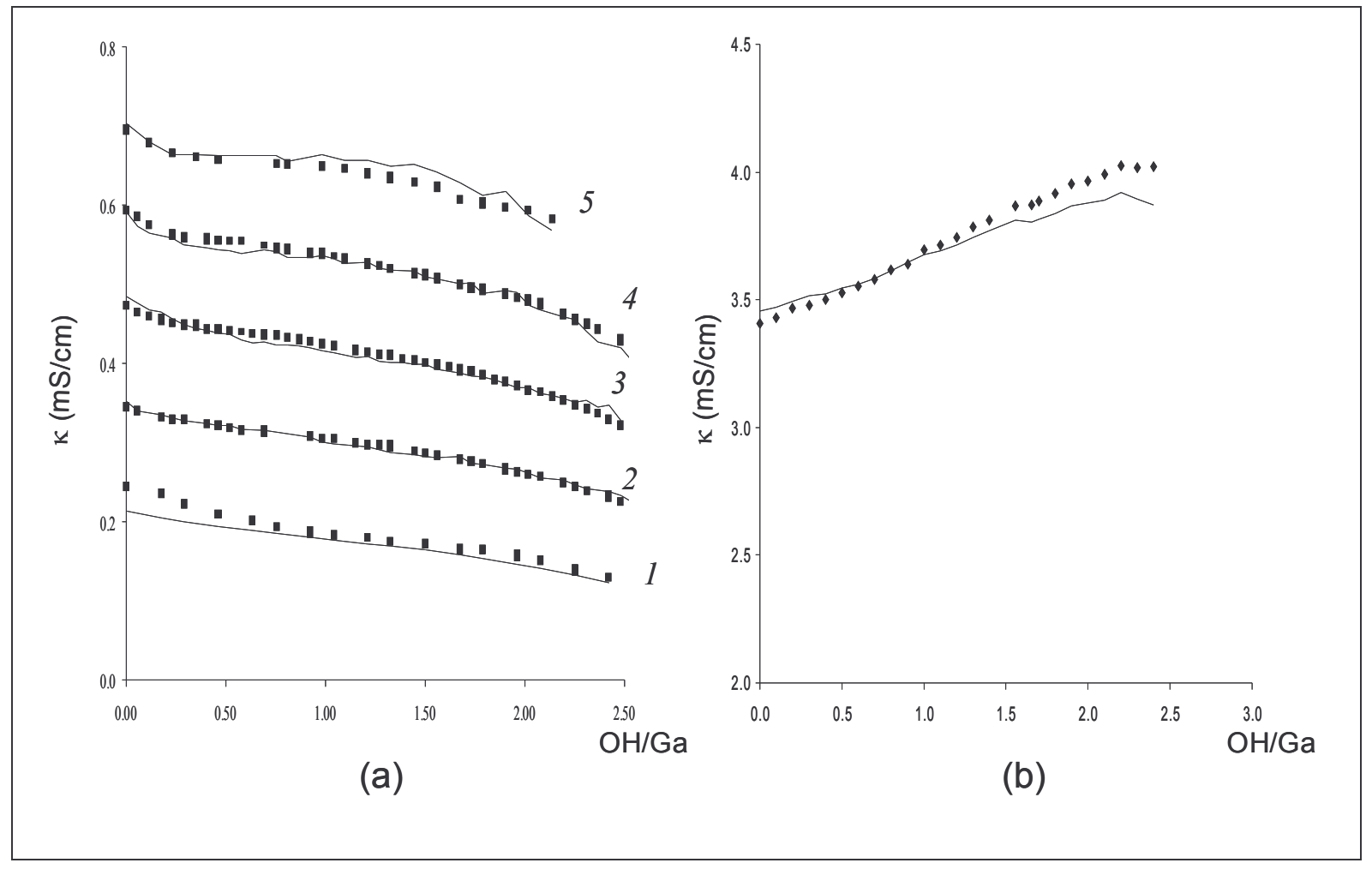

Figura 1.21. Valoraciones conductimétricas para el sistema $\mathrm{Ga}(\mathrm{III})-\mathrm{H}_{2} \mathrm{O}$.

(a) (1) $[\mathrm{Ga}(\mathrm{III})]_{\mathrm{T}}=3.2\left(10^{-4}\right) \mathrm{M}$, (2) $[\mathrm{Ga}(\mathrm{III})]_{\mathrm{T}}=6.4\left(10^{-4}\right) \mathrm{M}$, (3) $[\mathrm{Ga}(\mathrm{III})]_{\mathrm{T}}=9.6\left(10^{-4}\right) \mathrm{M}$, (4) $[\mathrm{Ga}(\mathrm{III})]_{\mathrm{T}}$ $=12.8\left(10^{-4}\right) \mathrm{M}$, (5) $\quad[\mathrm{Ga}(\mathrm{III})]_{\mathrm{T}}=16.0\left(10^{-4}\right) \quad$ (b) $[\mathrm{Ga}(\mathrm{III})]_{\mathrm{T}}=0.01 \mathrm{M}$. Las líneas representan la conductividad estimada empleando el modelo obtenido en la Tabla 2.12 por medio del programa SUPERQUAD. Los marcadores representan los valores de conductividad experimentales

$$
\begin{gathered}
\kappa=|z| \lambda_{\mathrm{Na}^{+}}\left[\mathrm{Na}^{+}\right]+|z| \lambda_{\mathrm{NO}^{-}}\left[\mathrm{NO}_{3}^{-}\right]+|z| \lambda_{\mathrm{H}^{+}}\left[\mathrm{H}^{+}\right]+|\mathrm{z}| \lambda_{\mathrm{OH}^{-}}\left[\mathrm{OH}^{-}\right]+ \\
\sum \mathrm{z} \mid \lambda_{\mathrm{Ga}_{\mathrm{i}}\left(\mathrm{OH}_{\mathrm{k}}^{3 \mathrm{i}-\mathrm{k}}\right.} \mathrm{F}_{\mathrm{Ga}_{\mathrm{i}}\left(\mathrm{OH}_{\mathrm{k}}{ }_{\mathrm{k}}^{3-\mathrm{k}}\right.}[\mathrm{Ga}(\mathrm{III})]_{\text {total }}
\end{gathered}
$$


Tabla 1.13. Valores estimados de Conductividad equivalente para el ajuste de las curvas de valoración conductimétricas para el sistema $\mathrm{Ga}(\mathrm{III})-\mathrm{H}_{2} \mathrm{O}$.

\begin{tabular}{ccc}
\hline Sistema & Especies & $\lambda / \mathrm{mS} \mathrm{cm}^{2}$ equivalente $^{-1}$ \\
\hline $\mathrm{Ga}(\mathrm{III})-\mathrm{H}_{2} \mathrm{O}$ & $\mathrm{Ga}^{3+}$ & $37-52$ \\
& $\mathrm{Ga}(\mathrm{OH})_{2}{ }^{+}$ & $15-20$ \\
& $\mathrm{Ga}(\mathrm{OH})_{3}$ & 0 \\
$\mathrm{Na}^{+}$ & $40-50$ \\
& $\mathrm{NO}_{3}{ }^{-}$ & $50-70$ \\
\hline
\end{tabular}

En la Figura 1.21 se muestran con línea continua las conductividades determinadas con el modelo de SUPERQUAD y con marcadores las conductividades equivalentes registradas experimentalmente Existe una adecuada sobreposición entre éstas, lo cual nos da argumentos para establecer la validez del modelo químico propuesto en la Tabla 1.11, ya que este nos permite explicar adecuadamente el comportamiento de las curvas conductimétricas observadas. Por otro lado, como ya se había reportado anteriormente ${ }^{43}$ dicho modelo explica también el comportamiento de los espectros de RMN de ${ }^{71}$ Ga durante el proceso de hidrólisis, sin embargo la realización de un estudio cuantitativo para ${ }^{71} \mathrm{Ga}-$ RMN, como se efectuó en el caso del sistema $\mathrm{Al}(\mathrm{III})-\mathrm{H}_{2} \mathrm{O}$, no es posible llevarlo a cabo debido a que la obtención de diversas áreas, para relacionarlas con las especies químicas, a partir de las señales de estos espectros es sumamente complicada por lo ancho y poco definidas que se tienen estas, además de que en un amplio intervalo de pH no se tiene señal alguna, lo cual dificultaría aún más el tratamiento cuantitativo 


\subsubsection{CONCLUSIONES}

El procesamiento de la información potenciométrica nos da como resultado un modelo para el sistema $\mathrm{Ga}(\mathrm{III})-\mathrm{H}_{2} \mathrm{O}$ en el que solamente se forman hidroxo-complejos de $\mathrm{Ga}$ (III) de tipo mononuclear (Tabla 1.11). Dicho modelo fue validado mediante la determinación de la función de formación (Z), además de que permite explicar satisfactoriamente el comportamiento de las curvas de valoración conductimétricas para este sistema. Se considera que los resultados que se muestran en el presente trabajo dan evidencia suficiente para considerar válido el modelo químico presentado en la Tabla 1.11, ya que esta basado no sólo en una técnica experimental, sino que el modelo propuesto explica satisfactoriamente el comportamiento experimental observado en potenciometría, conductimetría y el determinado en $\mathrm{RMN}^{48}$.

\subsection{SISTEMA In(III)- $\mathrm{H}_{2} \mathrm{O}$}

\subsubsection{ANTECEDENTES}

Probablemente de los elementos del grupo IIIA el que ha recibido menor atención sobre el estudio de las especies que se pueden formar en el proceso de hidrólisis en solución acuosa es el indio(III). Existen muy pocos estudios sobre las especies y sus constantes de formación los cuales en su mayoría reportan la formación de especies mononucleares que 
van desde la estequiometría $(1,1)$ hasta la $(1,4)$, así como la posible formación de especies polinucleares cuya estequiometría no esta bien definida ya que se ha propuesto desde la posible formación de un dímero hasta la de un tetramero, sin que ninguna de estas quede totalmente elucidada hasta el momento. Además, muchos de estos modelos datan de los años cincuentas y sesentas por lo que se puede suponer que quizás tanto las estequiometrías de las especies químicas, así como sus constantes de formación fueron probablemente determinadas por métodos gráficos. Lo anterior no resta importancia a los estudios, sin embargo, permite pensar que estos no se realizaron con la precisión adecuada. En la Tabla 1.14 se muestran algunos de los modelos reportados para el sistema $\mathrm{In}(\mathrm{III})-\mathrm{H}_{2} \mathrm{O}$ en la que pueden apreciarse no sólo la diversidad de modelos sino también la inconsistencia que se tiene para los valores reportados de las constantes de formación aún para una misma especie.

Tabla 1.14. Modelos de constantes de formación propuestos para la hidrólisis de In(III)

\begin{tabular}{|l|l|c|c|}
\hline \multicolumn{1}{|c|}{ AUTOR(ES) } & $\begin{array}{l}\text { Especie } \\
\text { Química }\end{array}$ & $-\log \boldsymbol{\beta}^{*}$ & Método \\
\hline Rossotti F. y Rossotti H. $^{59}$ & $\mathrm{In}(\mathrm{OH})^{2+}$ & 4.4 & Potenciometría \\
& $\mathrm{In}(\mathrm{OH})_{2}{ }^{+}$ & 8.8 & \\
\hline Biedermann G., Li N., Yu J. ${ }^{60}$ & $\mathrm{In}(\mathrm{OH})^{2+}$ & 6.95 & Potenciometría \\
\hline \multirow{3}{*}{ Baes C.F. y Mesmer R.E. ${ }^{1+}$} & $\operatorname{In}_{2}(\mathrm{OH})_{2}{ }^{3+}$ & 10.15 & \\
& $\operatorname{In}(\mathrm{OH})^{2+}$ & 4.00 & \\
& $\operatorname{In}(\mathrm{OH})_{2}{ }^{+}$ & 7.82 & Potenciometría \\
& $\operatorname{In}(\mathrm{OH})_{3}$ & 12.40 & \\
& $\operatorname{In}(\mathrm{OH})_{4}{ }^{-}$ & 22.07 & \\
& $\operatorname{In}(\mathrm{OH})_{4}{ }^{5+}$ & 5.82 & \\
\hline
\end{tabular}


Tabla 1.14 Modelos de constantes de formación propuestos para la hidrólisis de In(III) (Continuación)

\begin{tabular}{|l|c|c|c|}
\hline Aziz A. y Lyle S. ${ }^{61}$ & $\mathrm{In}(\mathrm{OH})^{2+}$ & 9.59 & Dispersión \\
& $\mathrm{In}(\mathrm{OH})_{2}{ }^{+}$ & 19.43 & \\
\hline Biedermann G., Ferri D. $^{62}$ & $\mathrm{In}(\mathrm{OH})^{2+}$ & 4.23 & \\
& $\mathrm{In}_{2}(\mathrm{OH})_{2}{ }^{3+}$ & 5.27 & Potenciometría \\
& $\mathrm{In}_{4}(\mathrm{OH})_{6}{ }^{6+}$ & 13.79 & \\
\hline Brown P., Ellis J y Sylva R. ${ }^{63}$ & $\mathrm{In}(\mathrm{OH})^{2+}$ & 4.31 & \\
& $\mathrm{In}_{2}(\mathrm{OH})_{2}{ }^{3+}$ & 9.33 & \multirow{2}{*}{ Potenciometría } \\
& $\mathrm{In}_{4}(\mathrm{OH})_{4}{ }^{8+}$ & 7.32 & \\
\hline
\end{tabular}

\subsubsection{EXPERIMENTAL}

\subsubsection{Estudios Potenciométricos}

Soluciones acuosas de $\mathrm{In}(\mathrm{III})$ dentro de un intervalo de $2.0 \times 10^{-3} \mathrm{M}$ a $10.0 \mathrm{X} 10^{-3}$ M a partir de cloruro de indio (Sigma 99.999\%) fueron preparadas. En la preparación de las soluciones se empleo agua desionizada y descarbonatada de Tipo I (desionizador Millipore). La concentración analítica de In (III) se determinó complejométricamente ${ }^{57}$.

La valoración de al menos dos muestras de cada solución fue llevada a cabo con soluciones estandarizadas de hidróxido de sodio libres de carbonatos (preparadas de acuerdo a lo indicado en la sección 1.1.2.1), en todos los casos se registraron al menos 60 datos por valoración. Las variaciones de $\mathrm{pH}$ y de potencial durante la valoración se midieron empleando un $\mathrm{pH}$-metro Mettler Toledo $(\Delta \mathrm{pH}=0.001)$, empleando un electrodo 
de vidrio combinado con referencia interna de $\mathrm{Ag} / \mathrm{AgCl}_{(\mathrm{s})}$; efectuando la calibración del equipo con soluciones buffer de referencia de $\mathrm{pH}=4.005$. Todas las valoraciones se realizaron manteniendo la temperatura constante a $25.0^{\circ} \mathrm{C}\left(\Delta \mathrm{T} \pm 0.1^{\circ} \mathrm{C}\right)$ por medio de un baño (VWR, modelo 12101-10, ) y empleando celdas de vidrio de doble camisa. Un flujo constante de nitrógeno de alta pureza se hizo pasar en las soluciones, durante todos los experimentos de potenciometría, con la finalidad de evitar la carbonatación del sistema

\subsubsection{Estudios de Resonancia Magnética Nuclear (RMN)}

Soluciones acuosas de $\operatorname{In}(\mathrm{III})$ $0.01 \mathrm{M}$ fueron preparadas a partir de cloruro de indio (Sigma 99.999\%). Las soluciones de In(III) se hidrolizaron con una solución acuosa de hidróxido de sodio estandarizada hasta obtener una relación $\mathrm{OH} / \mathrm{M}$ de 0.15 que es a partir de la cual el parámetro de $\mathrm{pH}$ comienza a presentar inestabilidad en función del tiempo. En todos los casos los valores de $\mathrm{pH}$ y de potencial fueron registrados a una temperatura de 25 ${ }^{\mathrm{o}} \mathrm{C}$.

Mediante un espectrofotometro Bruker DMX-500 se determinaron los espectros de $\mathrm{RMN}$ de ${ }^{115} \mathrm{In}$ a $25{ }^{\circ} \mathrm{C}$ empleando $\mathrm{In}\left(\mathrm{H}_{2} \mathrm{O}\right)_{6}{ }^{3+}$ como referencia de desplazamiento químico, la frecuencia de excitación usada para el núcleo de indio fue igual a 109.60 MHz.

Para la obtención de los espectros, en los estudios de RMN realizados para todos los cationes (Al(III), Ga(III), B(III) e In(III)) se adicionó $\mathrm{D}_{2} \mathrm{O}$ a cada sistema con la finalidad 
de fijar la señal magnética de campo externo. El porcentaje de incertidumbre para el desplazamiento químico observado $\left(\%\left(\Delta \delta_{0} / \delta_{0}\right)\right)$ no fue mayor al $1 \%$. En los casos en que fue posible se realizó la determinación del área correspondiente a cada señal, mediante la integración correspondiente para lo cual se empleó un porcentaje de incertidumbre $(\%(\Delta \mathrm{A} / \mathrm{A}))$ en un intervalo de $2.0 \%$ a $5.0 \%$, cuando se aplica el algoritmo de deconvolución del equipo. En todos los casos para la preparación de soluciones y para los experimentos se empleó agua desionizada y descarbonatada Tipo I (Millipore). Los sistemas se burbujearon con nitrógeno con la finalidad de evitar la carbonatación.

\subsubsection{RESULTADOS Y ANÁLISIS}

\subsubsection{Estudios Potenciométricos}

La hidrólisis de soluciones de indio(III) no resulta fácil de realizar ya que, si bien se tiene una zona de $\mathrm{pH}$ en la que este parámetro es estable, después de aproximadamente una relación $\mathrm{OH} / \mathrm{M}=0.15$ comienza a observarse una disminución del $\mathrm{pH}$, siendo la medición de este difícil por la falta de estabilidad en la lectura, este comportamiento puede ser debido a la posible aparición del precipitado de hidróxido de indio. En la Figura 1.22 se puede apreciar la curva de valoración para la hidrólisis de una solución de indio(III) de concentración $0.01 \mathrm{M}$ realizando las lecturas a intervalos de tiempo definidos de 10 minutos, se pudo constatar que existía reproducibilidad en las curvas obtenidas, sin embargo el comportamiento observado y hasta ahora no reportado representa una dificultad para el procesamiento de la información con el programa 


\section{SUPERQUAD.}

Baes y Mesmer $^{1}$ reportan que la precipitación del hidróxido de indio ocurre en relaciones molares menores a 0.7 , sin embargo no especifican el valor preciso de esta. En este trabajo tomando como hipótesis lo planteado por Baes y Mesmer ${ }^{1}$, es decir pensando que la poca estabilidad y la disminución de $\mathrm{pH}$ se pudiese deber a fenómenos de precipitación se realizó la determinación de los espectros de absorción para diferentes grados de hidrólisis de indio, sin embargo en ellos no es posible evidenciar la aparición de algún precipitado, dado que no se observa un incremento en la relación señal/ruido que sugiera la posible difracción o reflexión de la luz en los espectros obtenidos debido a partículas en suspensión (Figura 1.23).

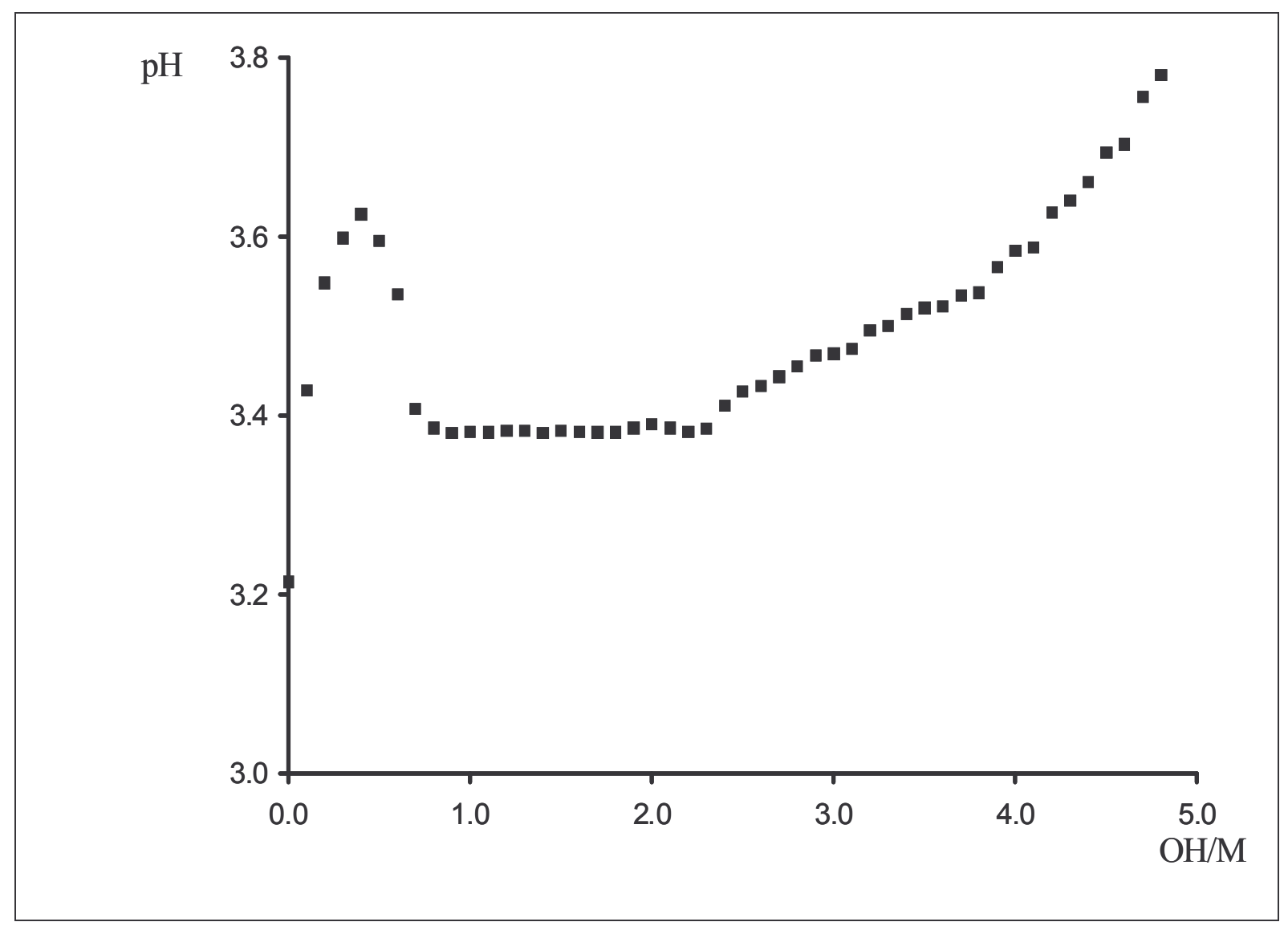

Figura 1.22. Curva de valoración para la hidrólisis de una solución de indio(III) de 
concentración $0.01 \mathrm{M}$ realizando las lecturas a intervalos de tiempo definidos de 10 minutos.

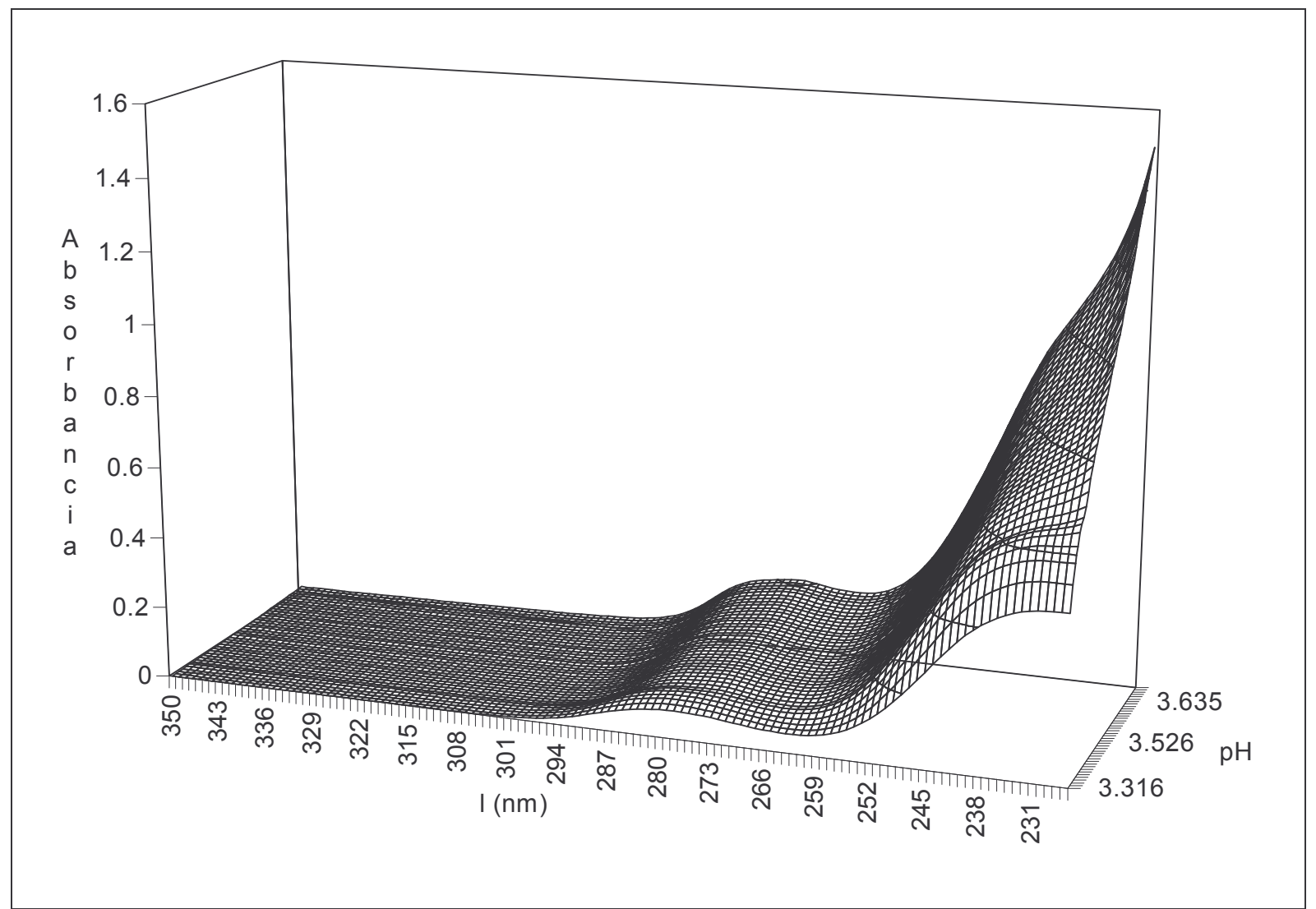

Figura 1.23. Espectrograma de absorción para diferentes grados de hidrólisis $(0.0 \leq \mathrm{OH} / \mathrm{M} \leq 2.5)$ de una solución de Indio(III) $0.01 \mathrm{M}$.

Al hacer una ampliación en la escala de los espectros de absorción característicos para el proceso de hidrólisis, hasta antes de que ocurra la inestabilidad del pH (Figura 1.24) es posible apreciar que la banda de absorción con un máximo en $278 \mathrm{~nm}$ presenta un incremento en su absortividad conforme el $\mathrm{pH}$ del sistema se incrementa, lo cual sugiere que en el sistema ocurre el proceso de hidrólisis y la transformación de especies químicas. 


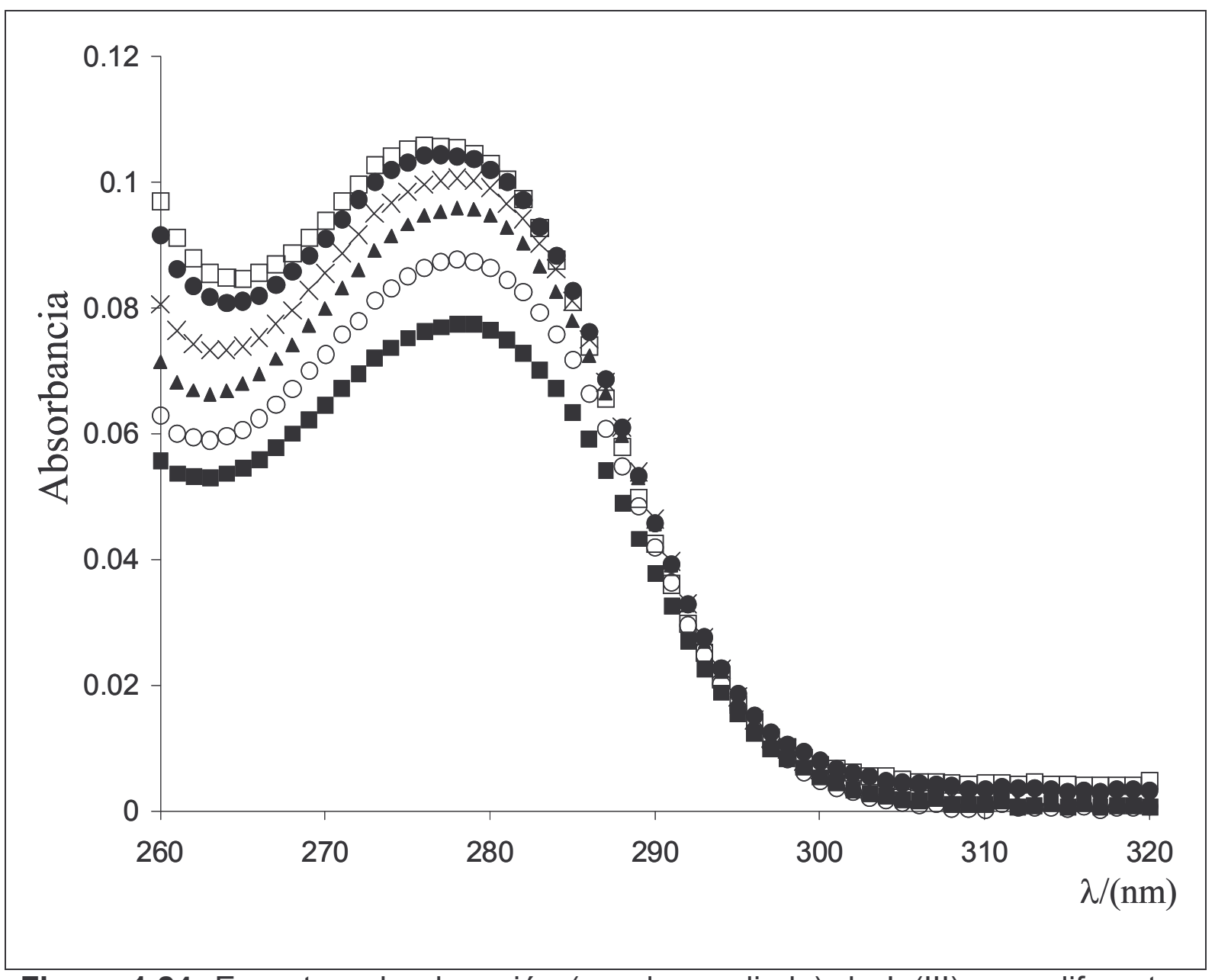

Figura 1.24. Espectros de absorción (escala ampliada) de $\ln (\mathrm{III})$ para diferentes

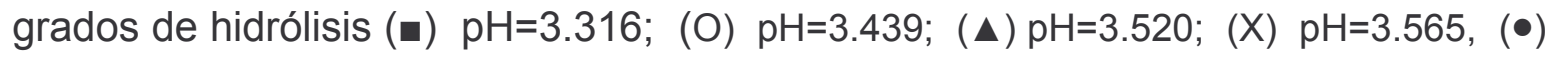
$\mathrm{pH}=3.582$, (口) $\mathrm{pH}=3.593$.

Considerando lo anterior se determinaron las curvas de hidrólisis para soluciones de indio(III) en un intervalo de $0.0 \leq \mathrm{OH} / \mathrm{M} \leq 0.18$. Después de este último valor la lectura de pH comienza a ser inestable y a disminuir. En la Figura 1.25 se observan las curvas obtenidas, en ellas se puede apreciar un ligero incremento del $\mathrm{pH}$ conforme el grado de hidrólisis aumenta. 


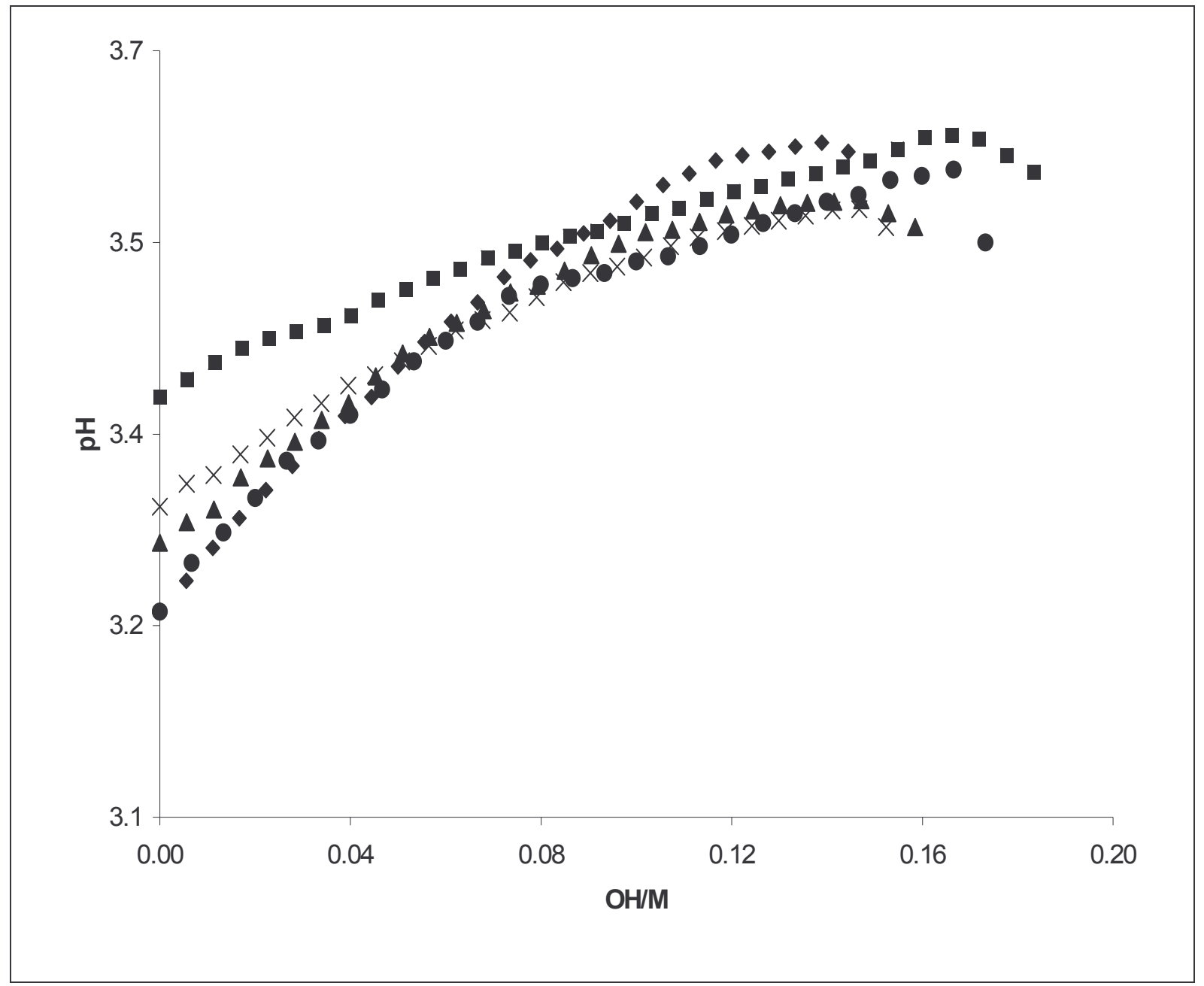

Figura 1.25 Curvas de valoración para soluciones de indio(III)

Los datos obtenidos de las valoraciones potenciométricas fueron alimentados al programa SUPERQUAD para el refinamiento de las constantes de equilibrio. Para ello se tomaron en consideración a especies monoméricas y poliméricas en relación con el equilibrio de la ecuación 1.15

$$
\begin{array}{r}
\mathrm{inn}^{3+}+\mathrm{jH}_{2} \mathrm{O}=\operatorname{In}_{\mathrm{i}}(\mathrm{OH})_{\mathrm{j}}^{3 \mathrm{i}-\mathrm{j}}+\mathrm{jH}^{+} \\
* \beta_{\mathrm{ij}}=\frac{\left[\operatorname{In}_{\mathrm{i}}(\mathrm{OH})_{\mathrm{k}}^{3 \mathrm{i}-\mathrm{k}}\right]\left[\mathrm{H}^{+}\right]^{\mathrm{j}}}{\left[\mathrm{In}^{3+}\right]^{\mathrm{i}}}
\end{array}
$$


Los mejores modelos obtenidos para el refinamiento de los datos se muestran en la Tabla 1.14, en ella se puede apreciar que la estadística obtenida para ellos es similar, lo cual no nos permite realizar la discriminación adecuada de los modelos.

Tabla 1.14 Valores de las constantes de formación para los refinamientos obtenidos a partir de datos potenciométricos procesados con SUPERQUAD para el sistema $\mathrm{In}(\mathrm{III})-\mathrm{H}_{2} \mathrm{O}^{\mathrm{a}}$.

\begin{tabular}{|c|c|c|c|}
\hline Modelo & Especies & $-\log * \beta$ & $\begin{array}{l}\text { Parámetros } \\
\text { Estadísticos }\end{array}$ \\
\hline 1 & $\begin{array}{c}\operatorname{In}(\mathrm{OH})^{2+} \\
\operatorname{In}(\mathrm{OH})_{3}\end{array}$ & $\begin{array}{c}4.718 \pm 0.069 \\
11.826 \pm 0.034\end{array}$ & $\begin{array}{l}\sigma^{b}=4.613 \\
\chi^{2 c}=17.25\end{array}$ \\
\hline 2 & $\begin{array}{l}\mathrm{In}(\mathrm{OH})_{2}^{+} \\
\mathrm{In}(\mathrm{OH})_{4}^{-}\end{array}$ & $\begin{array}{c}8.116 \pm 0.035 \\
15.919 \pm 0.148\end{array}$ & $\begin{array}{l}\sigma^{\mathrm{b}}=4.646 \\
\chi^{2 \mathrm{c}}=12.94\end{array}$ \\
\hline 3 & $\begin{array}{c}\mathrm{In}(\mathrm{OH})^{2+} \\
\mathrm{In}_{3}(\mathrm{OH})_{4}^{5+}\end{array}$ & $\begin{array}{c}4.358 \pm 0.015 \\
11.920 \pm 0.116\end{array}$ & $\begin{array}{c}\sigma^{b}=4.650 \\
\chi^{2 c}=27.65\end{array}$ \\
\hline
\end{tabular}

\footnotetext{
${ }^{a}$ Refinamiento realizado con 280 datos de las valoraciones potenciométricas. ${ }^{b}$ Desviación estándar total para el refinamiento ${ }^{\mathrm{c}}$ Chi-cuadrada para el refinamiento.
}

Con la finalidad de poder establecer el mejor modelo químico, para el sistema In(III)- $\mathrm{H}_{2} \mathrm{O}$, a partir de los datos potenciométricos se determinaron los valores de la función de formación ${ }^{1}(\mathrm{Z})$ (Apéndice 1). En la Figura 1.26 se muestran las curvas de $\mathrm{Z}$ como función del $\mathrm{pH}$ del sistema, en la cual se puede apreciar que la función de formación presenta un comportamiento similar en todos los casos. Sin embargo, existe un desplazamiento en función de la concentración total de In(III) lo cual suele ser característico para sistemas en los cuales existen especies polinucleares. Lo anterior nos 
permite proponer que el mejor modelo químico es aquel en el que se propone la formación de las especies $(1,1)$ y $(3,4)$ entre indio e hidróxido.

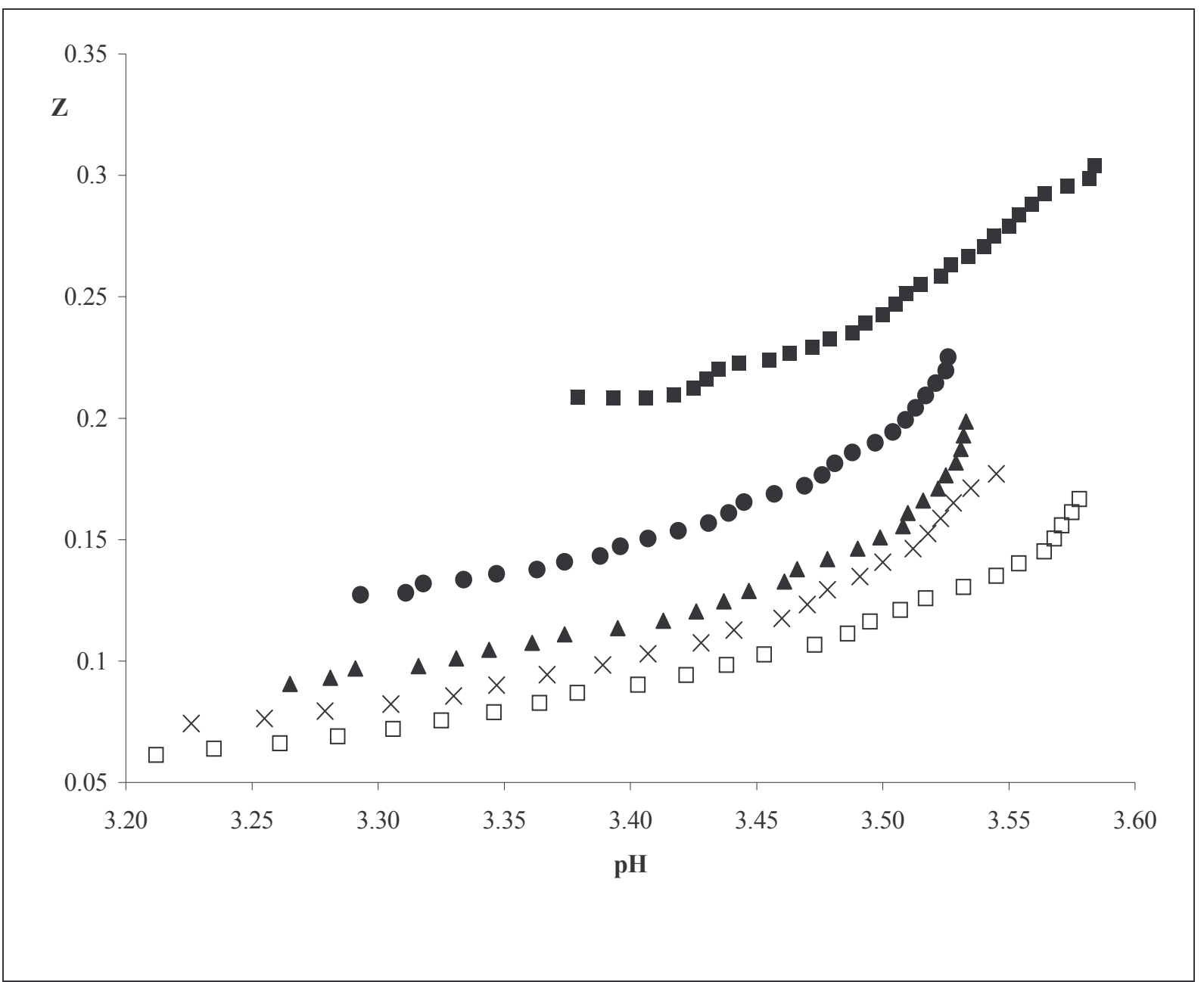

Figura 1.26. Gráfico de $\mathrm{Z}$ como función del $\mathrm{pH}$ para el sistema de $\mathrm{In}(\mathrm{III})-\mathrm{H}_{2} \mathrm{O}$, para diferentes

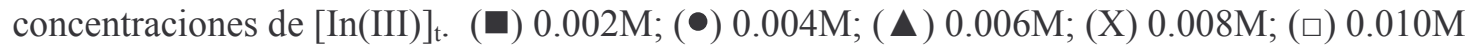

\subsubsection{Estudios de RMN}

Los espectros de ${ }^{115}$ In para dos diferentes grados de hidrólisis $(\mathrm{OH} / \mathrm{M}=0.0,0.60 \mathrm{y}$ 1.40) se muestran en la Figura 1.27, en ella se comparan dichos espectros con el obtenido para la referencia de $\mathrm{In}\left(\mathrm{H}_{2} \mathrm{O}\right)_{6}{ }^{3+}$ Como puede observarse la señal registrada es muy ancha, 
ya que esta comprende desde aproximadamente $-100 \mathrm{ppm}$ hasta $100 \mathrm{ppm}$, ello no permite apreciar las posibles diferentes geometrías de los núcleos de indio(III) en las diferentes especies. Por otro lado no se observa cambio o desplazamiento alguno de la señal en relación a la referencia empleada. Lo anterior nos permite establecer que en este caso las señales de resonancia magnética nuclear no permiten tener evidencia contundente para poder establecer que tipo de especies se pudieran estar formando durante el proceso de hidrólisis de soluciones de indio.

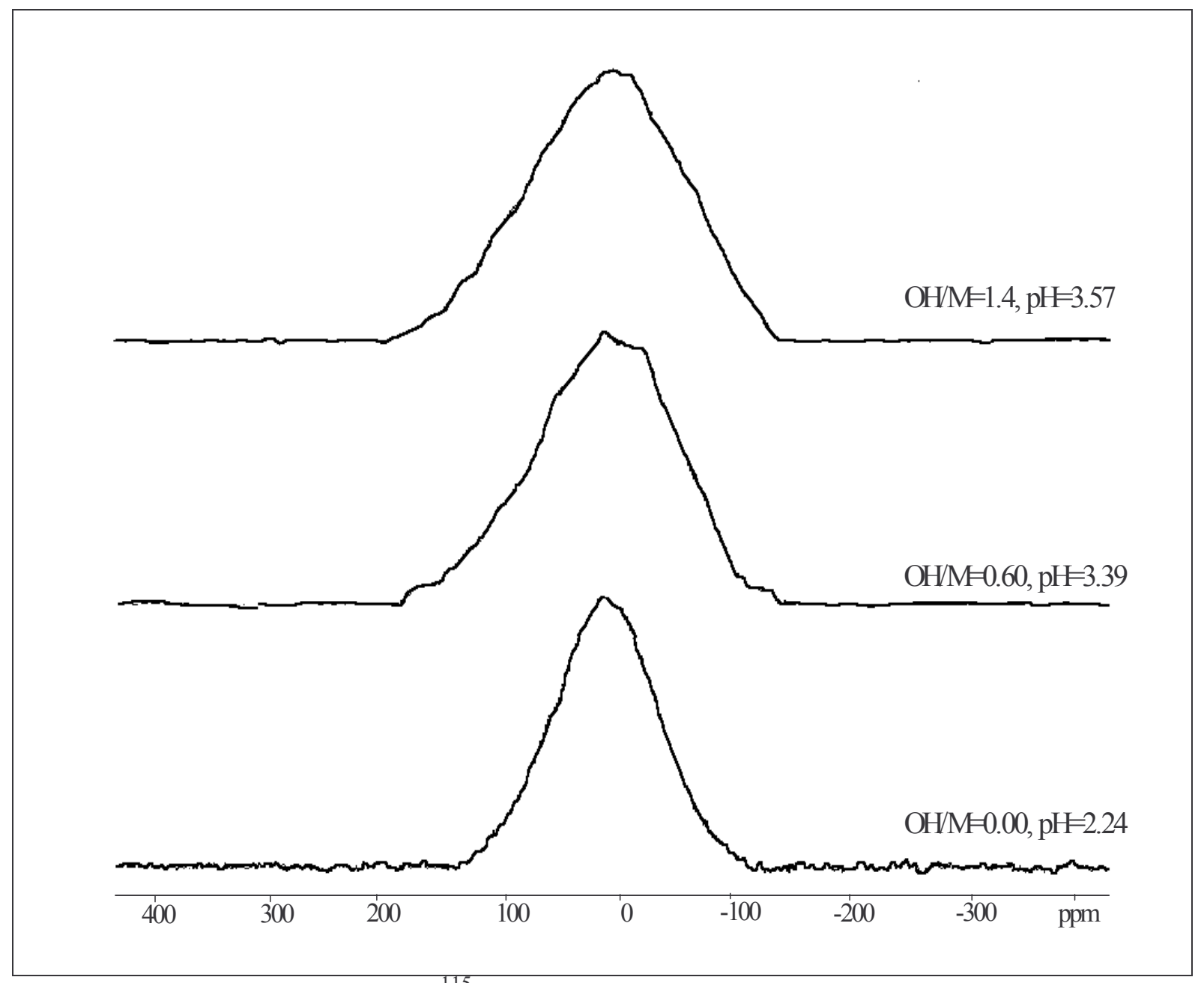

Figura 1.27. Espectros de RMN de ${ }^{115}$ In para el sistema $\operatorname{In}(\mathrm{III})-\mathrm{H}_{2} \mathrm{O}$. [In(III) $]=0.10 \mathrm{M}$ 


\subsubsection{CONCLUSIONES}

Con base en los resultados y en la información obtenida en el estudio del sistema $\mathrm{In}(\mathrm{III})-\mathrm{H}_{2} \mathrm{O}$ se puede establecer que este sistema tiene un proceso de hidrólisis sumamente rápido y complejo de estudiar. Se logró determinar con base en los datos potenciométricos que después de una relación $\mathrm{OH} / \mathrm{M} \geq 0.15$ el $\mathrm{pH}$ del sistema es inestable y tiene un comportamiento muy particular ya que disminuye conforme se le adiciona hidróxido al sistema. Lo anterior sugiere que después de esta relación tiene lugar la posible formación de algún hidroxo-precipitado de indio como lo proponen Baes y Mesmer ${ }^{1}$.

En este trabajo se logró determinar tres modelos químicos posibles para el sistema, los cuales presentan una estadística similar, con lo cual se hace difícil el determinar entre ellos al modelo más probable. Sin embargo, en el estudio de la función de formación (Z) realizado se determinó un comportamiento similar al observado en aquellos sistemas que presentan en su proceso de hidrólisis la formación de especies polinucleares, por lo que se propone en este trabajo que el modelo más probable es aquel que lleva a la formación de las especies $\operatorname{In}(\mathrm{OH})^{2+}(\log * \beta=4.358 \pm 0.015)$ y $\operatorname{In}_{3}(\mathrm{OH})_{4}{ }^{5+}(\log * \beta=11.920 \pm 0.116)$. Sin embargo, este modelo no pudo ser validado mediante estudios de RMN debido a que la señal es demasiado ancha para evidenciar cambios de geometría en las posibles especies químicas. Por lo tanto se reporta a este como posible modelo, sin poder descartar del todo los otros dos modelos refinados con SUPERQUAD, ya que este sistema presenta una gran complejidad en su estudio lo cual no permite tener la suficiente evidencia experimental, 
para poder ser contundentes en la proposición de un modelo químico para el proceso de hidrólisis de In(III) 


\section{CAPÍTULO II}

\section{ESTUDIO Y CARACTERIZACIÓN DE LOS SISTEMAS TERNARIOS: $\mathrm{Al}(\mathrm{III})-\mathrm{B}(\mathrm{III})-\mathrm{H}_{2} \mathrm{O}, \mathrm{Al}(\mathrm{III})-\mathrm{In}(\mathrm{III})-\mathrm{H}_{2} \mathrm{O}$ Y Al(III)-Ga(III)-- ${ }_{2} \mathrm{O}$}

\section{INTRODUCCIÓN}

Si bien en el capítulo anterior se señaló la importancia de caracterizar a las especies químicas que se forman en soluciones empleadas para la impregnación de arcillas que se utilizan como catalizadores. también se mencionó que los estudios realizados a este respecto son muy pocos para sistemas binarios y en mucho menor medida se han realizado para sistemas ternarios.

Los sistemas ternarios pueden estar implícitos en diversas áreas de la ciencia y la tecnología, sin embargo estos pocas veces son estudiados, debido a la complejidad que esto representa, más aun los estudios reportados para sistemas en los que se tienen a dos cationes y un ligante son muy escasos.

En el desarrollo de sistemas de arcillas pilareadas empleados como catalizadores el análisis de sistemas ternarios puede adquirir especial interés. La interacción de dos cationes puede ser importante debido a que permiten una mayor exposición de los sitios ácidos así como el desarrollo de nuevos materiales mesoporosos para generar productos pilareados con alta estabilidad térmica ${ }^{9,11,12,64,65}$. 
En este trabajo se realiza un estudio sobre sistemas ternarios en los que se tiene la presencia de aluminio(III), con la finalidad de proporcionar información sobre posibles especies que puedan ser utilizadas como precursores de las especies que pudiesen impregnarse en arcillas empleadas como catalizadores.

\subsection{SISTEMA $\mathrm{Al}(\mathrm{III})-\mathrm{B}(\mathrm{III})-\mathrm{H}_{2} \mathrm{O}$}

\subsubsection{EXPERIMENTAL}

\subsubsection{Estudios Potenciométricos.}

Con el propósito de establecer si existe una interacción entre el Al(III) y B(III) se realizó un estudio potenciométrico de soluciones conteniendo a ambos cationes en concentraciones equimolares $(\mathrm{Al}(\mathrm{III}): \mathrm{B}(\mathrm{III})=1: 1)$ a partir de $\mathrm{Al}\left(\mathrm{NO}_{3}\right)_{3} 9_{9} \mathrm{H}_{2} \mathrm{O}($ Baker Analyzed, 99.1\%) y de $\mathrm{H}_{3} \mathrm{BO}_{3}$ (Merck 99.8\%). Se prepararon cinco concentraciones de las soluciones antes mencionadas $(0.01 \mathrm{M}$ a $0.1 \mathrm{M})$.

Dos muestras al menos de cada concentración fueron valoradas, registrando un mínimo de 50 puntos por valoración, con soluciones estandarizadas de hidróxido de sodio. En todos los casos se registraron las variaciones de $\mathrm{pH}$ y potencial con un $\mathrm{pH}$-metro Radiometer-Tacussel LPH430T $(\Delta \mathrm{pH}=0.001)$, empleando para ello un electrodo de vidrio combinado con referencia interna de $\mathrm{Ag} / \mathrm{AgCl}_{(\mathrm{s})}$. La calibración del equipo se realizó con soluciones buffer de referencia de $\mathrm{pH}=4.005$. En todos los casos se mantuvo constante la temperatura del sistema a $25^{\circ} \mathrm{C}\left(\Delta \mathrm{T}=0.1^{\circ} \mathrm{C}\right)$ con ayuda de un baño 
(VWR, modelo 12101-10) y empleando celdas de vidrio de doble camisa. Además se mantuvo un flujo constante de nitrógeno de alta pureza para evitar la carbonatación del sistema.

Para la preparación de todas las soluciones y en el desarrollo de los experimentos se empleo agua desionizada y descarbonatada Tipo I (desionizador Millipore).

\subsubsection{Estudios de RMN.}

Soluciones acuosas de $\mathrm{Al}(\mathrm{III})$ 0.1: $\mathrm{B}$ (III) $0.1 \mathrm{M}$ fueron preparadas a partir de $\mathrm{Al}\left(\mathrm{NO}_{3}\right)_{3} \bullet 9 \mathrm{H}_{2} \mathrm{O}$ (Sigma ACS, $99.1 \%$ ) y de $\mathrm{H}_{3} \mathrm{BO}_{3}$ (Merck 99.8\%). Dichas soluciones fueron hidrolizadas para alcanzar la relación $\mathrm{OH} / M(0.0 \leq \mathrm{OH} / M \leq 1.5)$, esto se consiguió por medio de una lenta adición de solución estándar de hidróxido de sodio, bajo agitación vigorosa y calentamiento ligero. En todos los casos el pH de la solución fue registrado.

Los espectros de RMN de ${ }^{27} \mathrm{Al} \mathrm{y}{ }^{11} \mathrm{~B}$ fueron determinados por medio de un espectrómetro Bruker DMX500 a una temperatura de $25.0^{\circ} \mathrm{C}$. Como referencias de desplazamiento químico se emplearon $\mathrm{Al}\left(\mathrm{H}_{2} \mathrm{O}\right)_{6}{ }^{3+}$ y $\mathrm{BF}_{3}-\mathrm{O}\left(\mathrm{C}_{2} \mathrm{H}_{5}\right)_{2}$ y se utilizó $\mathrm{D}_{2} \mathrm{O}$ para fijar la señal de campo externo, la excitación empleada para el caso de ${ }^{27} \mathrm{Al}$ fue de 130.32MHz, mientras que para ${ }^{11} \mathrm{~B}$ se empleo una excitación de $160.46 \mathrm{MHz}$. 


\subsubsection{RESULTADOS Y DISCUSIÓN}

\subsubsection{Estudios Potenciométricos}

En la Figura 2.1 se muestran las curvas características para la valoración del sistema $\mathrm{Al}(\mathrm{III})-\mathrm{B}(\mathrm{III})-\mathrm{H}_{2} \mathrm{O}$ estas exhiben un incremento notable del $\mathrm{pH}$ al inicio de la valoración (hasta una relación $\mathrm{OH} / \mathrm{M} \leq 0.10$ ) y un incremento más ligero del $\mathrm{pH}$ posteriormente, conforme la valoración va progresando, esto es hasta alcanzar $\mathrm{OH} / \mathrm{M}=1.2$ aproximadamente, por encima de la relación anterior ocurre la aparición de un precipitado con características físicas similares a las del precipitado de Al(III)

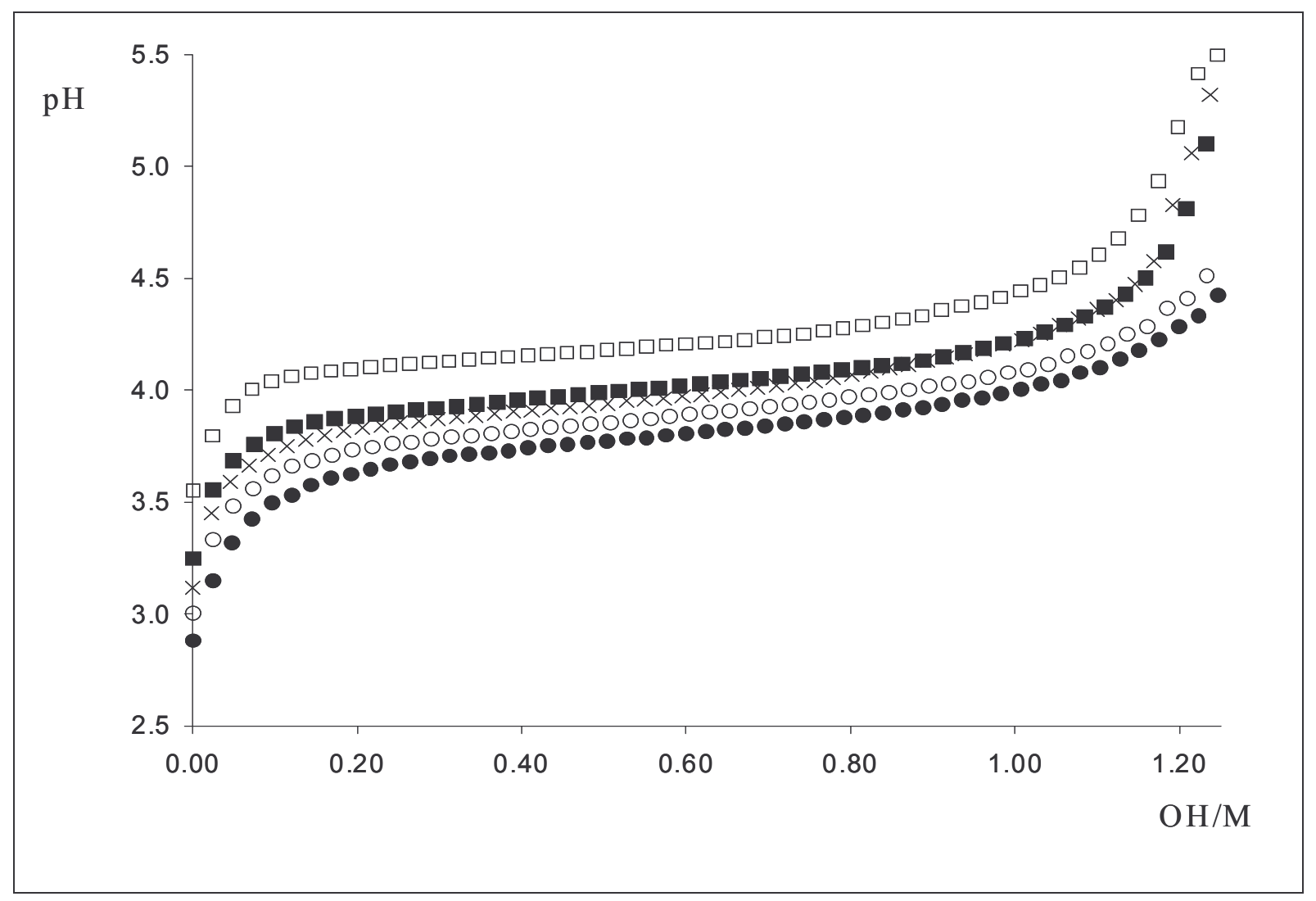


Figura 2.1. Curvas de valoración para el sistema $\mathrm{Al}(\mathrm{III})-\mathrm{B}(\mathrm{III})-\mathrm{H}_{2} \mathrm{O}$ a $25^{\circ} \mathrm{C}$ (口) $0.01 \mathrm{M} / 0.01 \mathrm{M}$; (ם) $0.03 \mathrm{M} / 0.03 \mathrm{M} ;(\mathrm{X})$ 0.05M/0.05M; (O) 0.07M/0.07M, (•) 0.10M/0.10M.

La forma que presentan estas curvas de valoración es muy similar a las del sistema $\mathrm{Al}(\mathrm{III})-\mathrm{H}_{2} \mathrm{O}$. En la Figura 2.2 se puede apreciar una comparación entre las curvas de valoración del sistema $\mathrm{Al}(\mathrm{III})-\mathrm{H}_{2} \mathrm{O}$ y $\mathrm{Al}(\mathrm{III})-\mathrm{B}(\mathrm{III})-\mathrm{H}_{2} \mathrm{O}$ para una concentración de $\mathrm{Al}(\mathrm{III})$ 0.1M. En ambos casos se observa la gran similitud que existe en el comportamiento gráfico de ambas valoraciones en el intervalo de $0.0 \leq \mathrm{OH} / \mathrm{M} \leq 0.90$. El incremento en los valores de pH en la valoración del sistema ternario puede deberse a la hidrólisis del ácido bórico (ver Figura 1.1).

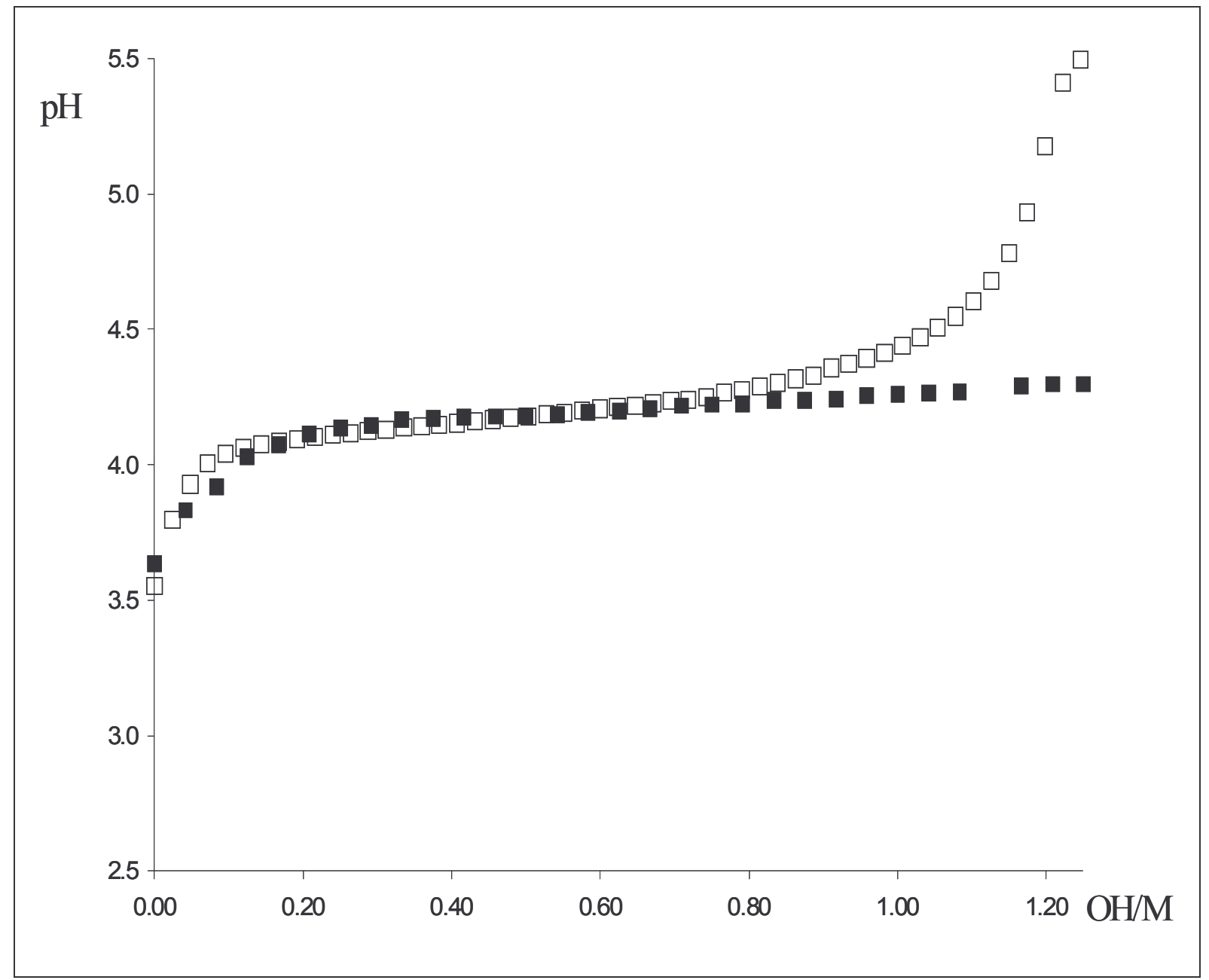

Figura 2.2. Comparación de las curvas de valoración para los sistemas $\mathrm{Al}(\mathrm{III})-\mathrm{H}_{2} \mathrm{O}$ ( $\boldsymbol{\square}$ ) y $\mathrm{Al}(\mathrm{III})-$ $\mathrm{B}(\mathrm{III})-\mathrm{H}_{2} \mathrm{O}(\square)$ a $25^{\circ} \mathrm{C}$ (en ambos casos la concentración de $\mathrm{Al}(\mathrm{III})$ es $0.1 \mathrm{M}$. 
Los datos obtenidos de las valoraciones potenciométricas fueron alimentados al programa computacional SUPERQUAD ${ }^{24,25}$ con la finalidad de determinar si existe una posible asociación química entre el $\mathrm{Al}(\mathrm{III})$ y $\mathrm{B}(\mathrm{III})$, de acuerdo a la ecuación 2.1. Sin embargo, durante el proceso de refinamiento no fue posible determinar alguna especie química que implicara una posible asociación química entre los metales antes mencionados. Los resultados obtenidos del mejor refinamiento se muestran en la Tabla 2.1, donde se observa que solamente se obtuvieron las constantes relacionadas con los hidroxocomplejos de $\mathrm{Al}(\mathrm{III})$ de estequiometría 1:1, 1:3, 3:4 y 13:32 así como la constante de hidrólisis de la especie $\mathrm{B}(\mathrm{OH})_{4}{ }^{-}$. El refinamiento obtenido en este caso da valores muy similares a los determinados en los sistemas binarios de $\mathrm{B}(\mathrm{III})-\mathrm{H}_{2} \mathrm{O}$ y $\mathrm{Al}(\mathrm{III})-\mathrm{H}_{2} \mathrm{O}$ (Tablas 1.2 y 1.5 del capítulo I). Lo anterior corrobora el análisis cualitativo realizado sobre las curvas de valoración obtenidas del sistema ternario y su semejanza en cuanto a comportamiento con las valoraciones obtenidas para $\mathrm{Al}(\mathrm{III})$ (Figura 2.2). El refinamiento solamente de la constante de disociación del monomero de $\mathrm{B}(\mathrm{III})$ se debe probablemente a que en el intervalo de $\mathrm{pH}$ analizado no existe información suficiente que permita el refinamiento de alguna especie polinuclear de boro.

$$
\begin{gathered}
i A l^{3+}+j B^{3+}+k H_{2} O=A l_{i} B_{j}(O H)_{k}^{3(i+j)-k}+k H^{+} \\
* \beta_{i j k}=\frac{\left[A l_{i} B_{j}(O H)_{k}^{3(i+j)-k}\right]\left[H^{+}\right]^{k}}{\left[A l^{3+}\right]\left[B^{3+}\right]^{j}}
\end{gathered}
$$


Tabla 2.1 Valores de las constantes de formación para el mejor refinamiento obtenido a partir de datos potenciométricos procesados con SUPERQUAD para el sistema $\mathrm{Al}(\mathrm{III})-\mathrm{B}(\mathrm{III})-\mathrm{H}_{2} \mathrm{O}$.

\begin{tabular}{ccc}
\hline Especies & $-\log * \beta$ & $\begin{array}{c}\text { Parámetros } \\
\text { Estadísticos }\end{array}$ \\
\hline $\mathrm{Al}(\mathrm{OH})^{2+}$ & $5.10^{\mathrm{a}}$ & $\sigma=1.768$ \\
$\mathrm{Al}(\mathrm{OH})_{3}$ & $13.22 \pm 0.04$ & $\mathbf{U}=\mathbf{1 6 2 1}$ \\
$\mathrm{Al}_{3}(\mathrm{OH})_{4}{ }^{5+}$ & $13.31 \pm 0.06$ & \\
$\mathrm{Al}_{13}(\mathrm{OH})_{32}{ }^{7+}$ & $108.60 \pm 0.17$ & \\
$\mathrm{~B}(\mathrm{OH})_{3}$ & $-9.30^{\mathrm{a}}$ &
\end{tabular}

${ }^{\mathrm{a}}$ Valor constante durante el refinamiento

\subsubsection{Estudios de RMN}

Al igual que en los estudios de potenciometría, las señales de RMN no muestran evidencia alguna de la posible interacción entre los iones metálicos de $\mathrm{Al}(\mathrm{III})$ y $\mathrm{B}(\mathrm{III})$ ya que en los espectros de resonancia magnética de ${ }^{27} \mathrm{Al}$ (Figura 2.3) se puede apreciar en el intervalo de $0.0 \leq \mathrm{OH} / \mathrm{M} \leq 1.15(2.761 \leq \mathrm{pH} \leq 3.921)$ una señal en 0 ppm la cual se ha determinado corresponde a núcleos con geometría octaédrica característica de las especies mononucleares $^{42,48}$. En el intervalo de $0.10 \leq \mathrm{OH} / \mathrm{M} \leq 0.95$ (3.27 $\left.\leq \mathrm{pH} \leq 3.550\right)$ se aprecia una señal que aparece con un desplazamiento químico de aproximadamente 5 ppm y que se ha relacionado con la especie polinuclear de bajo peso molecular $\mathrm{Al}_{3}(\mathrm{OH})_{4}{ }^{5+}$ (ver capítulo I) y finalmente en $0.15 \leq \mathrm{OH} / \mathrm{M} \leq 1.30(3.423 \leq \mathrm{pH} \leq 4.728)$ se tiene una señal en aproximadamente 63 ppm que se ha asignado a la especie $\mathrm{Al}_{13}(\mathrm{OH})_{32}{ }^{7+}$. 


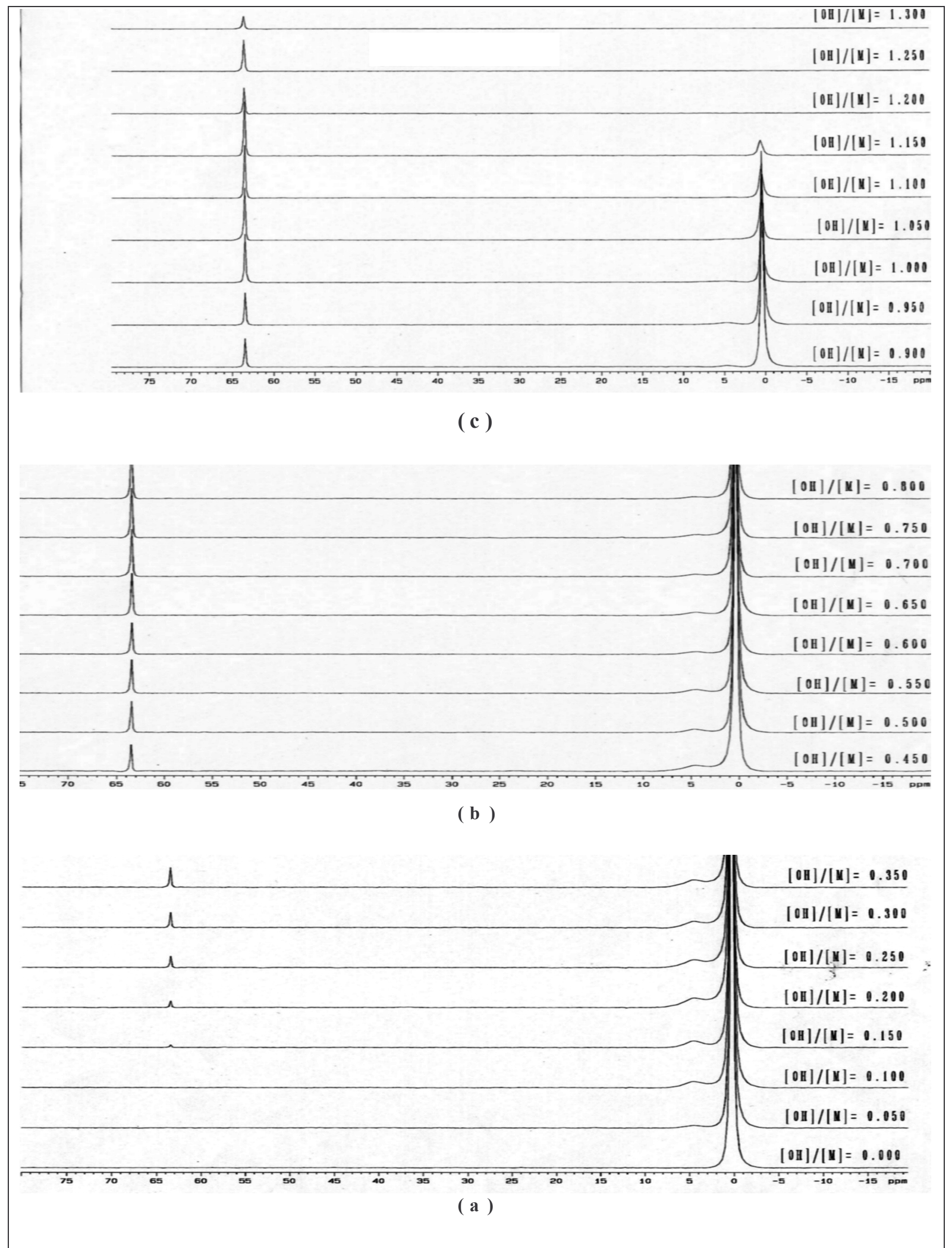

Figura 2.3. Espectros de $\mathrm{RMN}$ de ${ }^{27} \mathrm{Al}$ para el sistema $\mathrm{Al}(\mathrm{III})-\mathrm{B}(\mathrm{III})-\mathrm{H}_{2} \mathrm{O}$. [Al(III)]= $[\mathrm{B}(\mathrm{III})]=0.10 \mathrm{M}$ 
Por otro lado, los espectros de resonancia magnética nuclear de ${ }^{11} \mathrm{~B}$ mostraron en todos los casos una señal en 19 ppm que se relaciona con el ácido bórico ${ }^{27}$, esta señal permanece constante durante todo el proceso de hidrólisis del sistema, lo cual nos sugiere que no existen cambios geométricos en las especies de boro. Por lo tanto, considerando que el cambio de $\mathrm{pH}$ del sistema es pequeño, se puede asumir que no se lleva a cabo la formación de las especies polinucleares de $\mathrm{B}$ (III) por lo que en todo el intervalo de $\mathrm{pH}$ de trabajo predomina la especie de $\mathrm{B}(\mathrm{OH})_{3}$ como se puede apreciar en la Figura 2.4

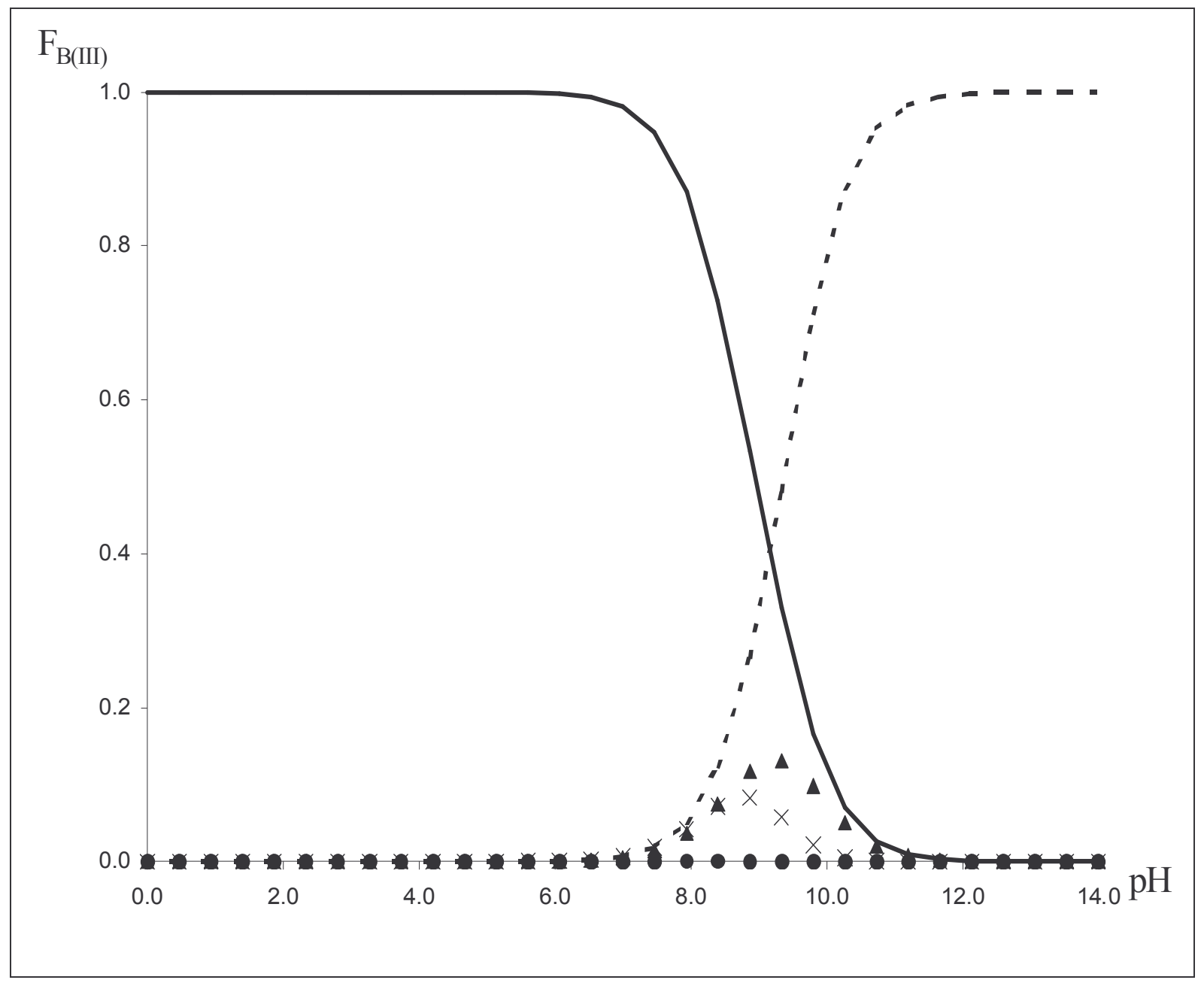

Figura 2.4. Diagrama de distribución de especies de $\mathrm{B}(\mathrm{III})$ para una concentración total de $0.1 \mathrm{M}$ $(-) \mathrm{B}(\mathrm{OH})_{3} ;(--) \mathrm{B}(\mathrm{OH})_{4}{ }^{-} ;(\boldsymbol{\Delta}) \mathrm{B}_{2} \mathrm{O}(\mathrm{OH})_{5}{ }^{-} ;(\mathrm{X}) \mathrm{B}_{3} \mathrm{O}_{3}(\mathrm{OH})_{4}{ }^{-} ;(\bullet) \mathrm{B}_{5} \mathrm{O}_{6}(\mathrm{OH})_{4}{ }^{-}$. 


\subsubsection{CONCLUSIONES}

Los estudios realizados para el sistema $\mathrm{Al}(\mathrm{III})-\mathrm{B}(\mathrm{III})-\mathrm{H}_{2} \mathrm{O}$ permiten establecer que no hay evidencia experimental suficiente para proponer la formación de un complejo ternario entre aluminio, boro e iones hidróxido. Se puede apreciar en los estudios potenciométricos que las curvas de valoración presentan un comportamiento muy similar a aquellas del sistema $\mathrm{Al}(\mathrm{III})-\mathrm{H}_{2} \mathrm{O}$. Se presenta un posterior incremento del $\mathrm{pH}$ el cual se ha atribuido a la hidrólisis del ácido bórico. Dado que no es posible seguir la valoración en relaciones mayores de $\mathrm{OH} / \mathrm{M}$ porque se presenta precipitación en el sistema de análisis no se tienen más datos del comportamiento del sistema. Asimismo con estos datos solo fue posible el refinamiento de las constantes de formación de los complejos entre aluminio e hidróxido, así como la constante de hidrólisis del ácido bórico a tetrahidroxoborato.

Por otro lado, los espectros de resonancia tanto de ${ }^{27} \mathrm{Al}$ como de ${ }^{11} \mathrm{~B}$ son similares a las que se tienen en los sistemas binarios. De haberse formado una asociación entre aluminio y boro se esperaría un desplazamiento de alguna o algunas de las señales o bien la desaparición de alguna de ellas y/o la aparición de una nueva señal. Sin embargo, esto no sucede, lo cual confirma lo antes planteado sobre la carencia de información experimental que permita reportar la formación de un complejo ternario entre aluminio(III)-boro(III)hidróxido. 


\subsection{SISTEMA Al(III)-In(III)- $\mathrm{H}_{2} \mathrm{O}$}

\subsubsection{EXPERIMENTAL}

\subsubsection{Estudios Potenciométricos.}

Se realizaron estudios potenciométricos con la finalidad de determinar si existe una posible interacción entre los iones metálicos de Al(III) y In(III). Para ello se prepararon cinco concentraciones diferentes de soluciones equimolares de estos iones metálicos a partir de $\mathrm{Al}\left(\mathrm{NO}_{3}\right)_{3} 9 \mathrm{H}_{2} \mathrm{O}$ (Baker Analyzed, 99.1\%) y de $\mathrm{InCl}_{3}(\mathrm{Sigma} 99.999 \%$ ).

De cada concentración se valoraron al menos dos muestras con soluciones estandarizadas de hidróxido de sodio, preparadas como se indica en 1.1.2.1. En cada valoración se registró un mínimo de 60 puntos por valoración. Tanto las variaciones de $\mathrm{pH}$ y de potencial fueron registradas con un $\mathrm{pH}$-metro Metler Toledo $(\Delta \mathrm{pH} \pm 0.001)$, empleando para ello un electrodo de vidrio combinado con referencia interna de $\mathrm{Ag} / \mathrm{AgCl}_{(\mathrm{s})}$. La calibración del equipo se realizó con sistemas buffer de referencia de $\mathrm{pH}=4.005$. La temperatura de experimentación se mantuvo como un parámetro constante en todos los casos con ayuda de un baño (VWR, modelo 12101-10) y empleando celdas de vidrio cerradas de doble camisa. Con la finalidad de evitar la carbonatación del sistema se mantuvo constante un flujo constante de nitrógeno de alta pureza. 
En el desarrollo de todos los experimentos y en la preparación de todas las soluciones se empleo agua destilada y descarbonatada Tipo I (desionizador Millipore).

\subsubsection{Estudios de RMN.}

Soluciones acuosas de Al(III) 0.1: In(III) $0.1 \mathrm{M}$ fueron preparadas a partir de $\mathrm{Al}\left(\mathrm{NO}_{3}\right)_{3} \cdot 9 \mathrm{H}_{2} \mathrm{O}$ (Sigma ACS, $99.1 \%$ ) y de $\mathrm{InCl}_{3}$ (Sigma 99.999). Estas soluciones fueron hidrolizadas con la lenta adición, con agitación vigorosa y calentamiento, de solución de hidróxido de sodio estándar para alcanzar la relación $\mathrm{OH} / M(0.0 \leq \mathrm{OH} / M \leq 2.5)$. En todos $\operatorname{los}$ casos el pH de la solución fue registrado.

Los espectros de $\mathrm{RMN}$ de ${ }^{27} \mathrm{Al}$ y ${ }^{115} \mathrm{In}$ fueron determinados por medio de un espectrómetro Bruker DMX500 a una temperatura de $25.0{ }^{\circ} \mathrm{C}$, utilizando $\mathrm{Al}\left(\mathrm{H}_{2} \mathrm{O}\right)_{6}{ }^{3+} \mathrm{y}$ $\mathrm{In}\left(\mathrm{H}_{2} \mathrm{O}\right)_{6}{ }^{3+}$ como referencia de desplazamiento químico y empleando una frecuencia de excitación de $130.32 \mathrm{MHz}$ y 109.60 para los núcleos de aluminio e indio, respectivamente. Se utilizó $\mathrm{D}_{2} \mathrm{O}$ para fijar la señal de campo externo.

\subsubsection{RESULTADOS Y DISCUSIÓN}

\subsubsection{Estudios Potenciométricos}

La Figura 2.5 muestra las curvas características para la valoración del sistema $\mathrm{Al}(\mathrm{III})-\mathrm{In}(\mathrm{III})-\mathrm{H}_{2} \mathrm{O}$. Se aprecia un notable incremento en el valor de $\mathrm{pH}$ hasta alcanzar una relación de $\mathrm{OH} / \mathrm{M}$ de aproximadamente 0.23 . Posterior a esta relación tiene lugar una 
disminución del $\mathrm{pH}$, además de ser inestable la lectura de este parámetro. Dicho comportamiento se relacionó en el capítulo I con la posible precipitación de un hidróxido de indio(III), sin embargo es importante destacar que a diferencia de las valoraciones del sistema $\mathrm{In}(\mathrm{III})-\mathrm{H}_{2} \mathrm{O}$ en este sistema ternario la disminución e inestabilidad del $\mathrm{pH}$ ocurre después de una relación $\mathrm{OH} / \mathrm{M}$ mayor a 0.23 , mientras que en el sistema binario esta tenía lugar en una relación posterior a 0.18 .

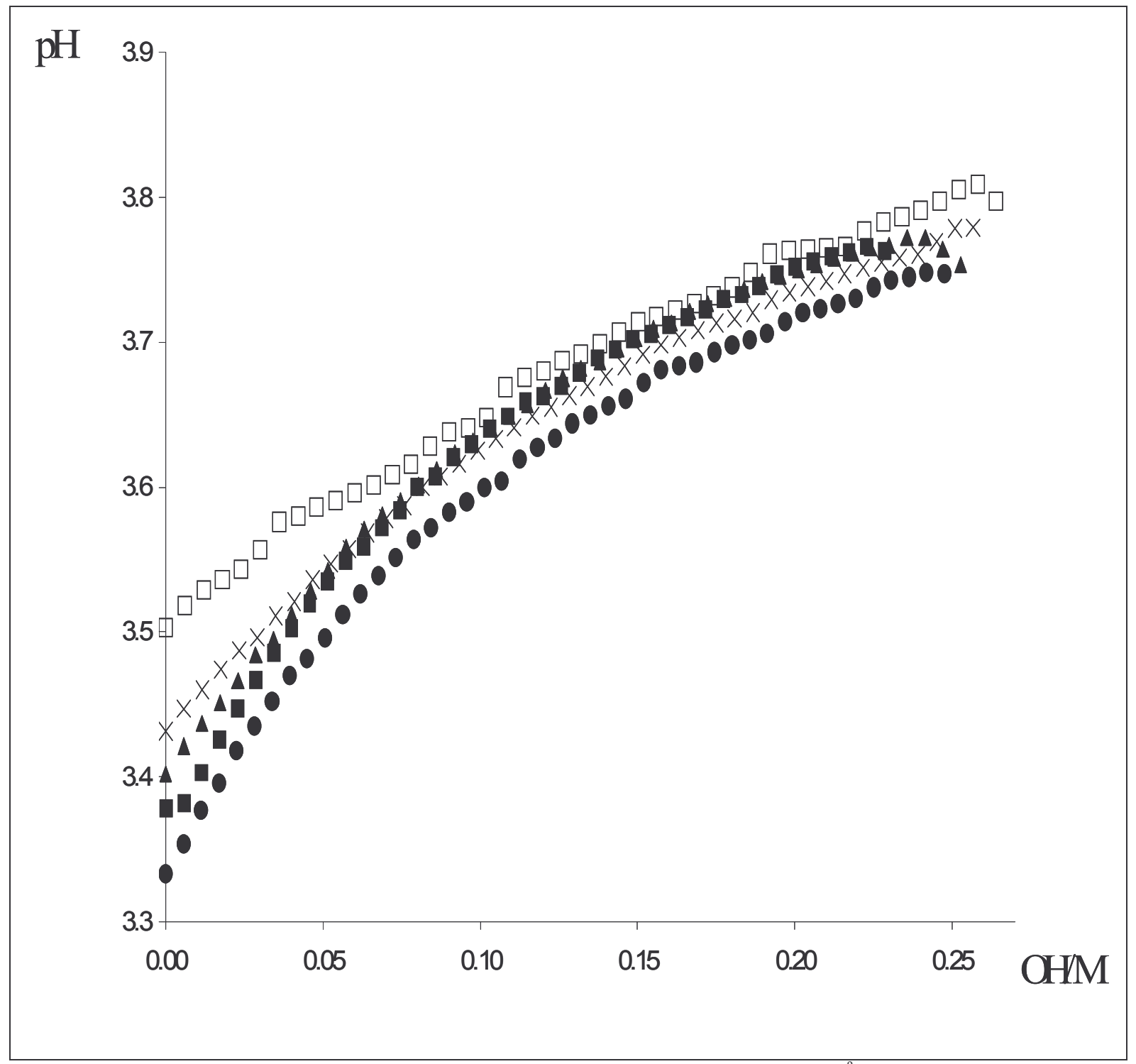

Figura 2.5. Curvas de valoración para el sistema $\mathrm{Al}(\mathrm{III})-\mathrm{In}(\mathrm{III})-\mathrm{H}_{2} \mathrm{O}$ a $25^{\circ} \mathrm{C}(\bullet) 0.1 \mathrm{M} / 0.1 \mathrm{M}$; (ם) 0.08M/0.08M; ( $\Delta$ ) 0.06M/0.06M; (X) 0.04M/0.04M; (口) 0.02M/0.02M. 
Por otro lado, en la Figura 2.6 se aprecia que en este caso las valoraciones del sistema $\mathrm{Al}(\mathrm{III})-\mathrm{In}(\mathrm{III})-\mathrm{H}_{2} \mathrm{O}$ tienen un comportamiento similar a aquellas de $\mathrm{In}(\mathrm{III})-\mathrm{H}_{2} \mathrm{O}$. A diferencia de lo que ocurrió con los sistemas de $\mathrm{Al}(\mathrm{III})-\mathrm{B}(\mathrm{III})-\mathrm{H}_{2} \mathrm{O}$ y $\mathrm{B}(\mathrm{III})-\mathrm{H}_{2} \mathrm{O}$. Lo anterior permite considerar que puede existir una interacción entre los iones metálicos $\mathrm{Al}(\mathrm{III})$ e In(III).

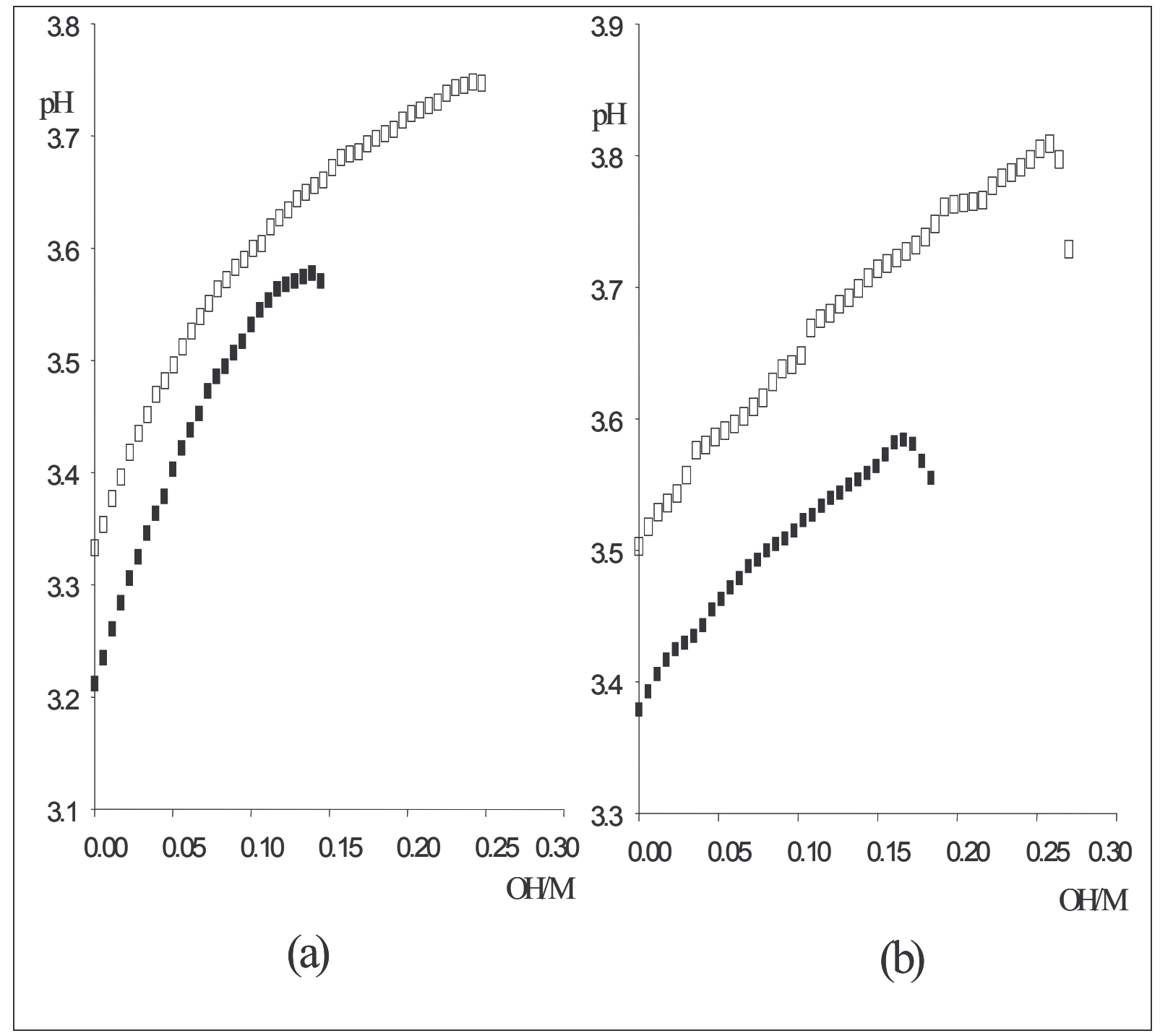

Figura 2.6. Comparación de las curvas de valoración para los sistemas $\mathrm{In}(\mathrm{III})-\mathrm{H}_{2} \mathrm{O}$ ( $\square$ ) y $\mathrm{Al}(\mathrm{III})-$ $\mathrm{In}(\mathrm{III})-\mathrm{H}_{2} \mathrm{O}$ (匹) a $25^{\circ}$ (a) $[\mathrm{In}(\mathrm{III})]=0.01 \mathrm{M}$; (b) $[\mathrm{In}(\mathrm{III})]=0.002 \mathrm{M}$; 
La información obtenida potenciométricamente fue alimentada al programa computacional SUPERQUAD con la finalidad de determinar la posible existencia de complejos ternarios formados entre aluminio(III), indio(III) e iones hidróxido de acuerdo a la ecuación 2.2.

$$
\begin{gathered}
i A l^{3+}+j I^{3+}+k H_{2} O=A l_{i} \operatorname{In}_{j}(\mathrm{OH})_{k}^{3(i+j)-k}+k H^{+} \\
* \beta_{i j k}=\frac{\left[A l_{i} \operatorname{In}_{j}(O H)_{k}^{3(i+j)-k}\right]\left[H^{+}\right]^{k}}{\left[A l^{3+}\right]\left[\operatorname{In}^{3+}\right]^{j}}
\end{gathered}
$$

Los mejores modelos obtenidos por medio de SUPERQUAD se muestran en la Tabla 2.2, donde se aprecia que los tres modelos químicos reportan valores consistentes con los descritos en las Tablas 1.5 y 1.14 (capítulo I). En todos los casos solamente fue posible el refinamiento considerando como constante el valor relacionado con la formación de $\mathrm{Al}(\mathrm{OH})^{3+}$ que es la especie predominante de $\mathrm{Al}(\mathrm{III})$ bajo las condiciones de trabajo. Sin embargo, para las especies de In(III) se presenta una situación similar a la descrita en el sistema $\mathrm{In}(\mathrm{III})-\mathrm{H}_{2} \mathrm{O}$ (ver capítulo I), donde no es posible distinguir el mejor modelo químico. 
Tabla 2.2 Valores de las constantes de formación para los refinamientos obtenidos a partir de datos potenciométricos procesados con SUPERQUAD para el sistema $\mathrm{Al}(\mathrm{III})-\mathrm{In}(\mathrm{III})-\mathrm{H}_{2} \mathrm{O}$.

\begin{tabular}{|c|c|c|}
\hline Especie & $-\log ^{*} \beta$ & $\begin{array}{l}\text { Parámetros } \\
\text { Estadísticos }\end{array}$ \\
\hline $\mathrm{Al}(\mathrm{OH})^{2+}$ & $5.29^{\mathrm{a}}$ & $\sigma=0.7340$ \\
\hline $\operatorname{In}(\mathrm{OH})^{2+}$ & $4.52 \pm 0.014$ & $\mathrm{U}=20.47$ \\
\hline $\mathrm{AlIn}_{2}(\mathrm{OH})_{4}{ }^{5+}$ & $12.27 \pm 0.068$ & \\
\hline $\mathrm{Al}(\mathrm{OH})^{2+}$ & $5.29^{\mathrm{a}}$ & $\sigma=0.2462$ \\
\hline $\operatorname{In}(\mathrm{OH})^{2+}$ & $4.88 \pm 0.019$ & $\mathrm{U}=23.02$ \\
\hline $\operatorname{In}(\mathrm{OH})_{3}$ & $12.45 \pm 0.012$ & \\
\hline $\mathrm{Al}(\mathrm{OH})^{2+}$ & $5.29^{\mathrm{a}}$ & $\sigma=0.2479$ \\
\hline $\operatorname{In}(\mathrm{OH})^{2+}$ & $8.46 \pm 0.010$ & $\mathrm{U}=23.68$ \\
\hline $\mathrm{In}(\mathrm{OH})_{4}^{-}$ & $17.08 \pm 0.10$ & \\
\hline
\end{tabular}

${ }^{\mathrm{a}}$ Valor constante durante el refinamiento

\subsubsection{Estudios de RMN}

Los espectros de resonancia magnética nuclear para ${ }^{27} \mathrm{Al}$ muestran una sola señal en 0 ppm la cual, como ya se explicó ampliamente en el capítulo I, está relacionada con núcleos de aluminio de geometría octaédrica. Este tipo de núcleos está presente en las especies monoméricas. La Figura 2.7 muestra un espectro de resonancia magnética cuando la relación $\mathrm{OH} / \mathrm{M}=0.0$ y cuando dicha relación es de 0.112 y se puede apreciar que la señal 
obtenida es prácticamente la misma, lo cual sugiere que no hay cambios en la geometría de los núcleos de aluminio. Con base en lo anterior solo se puede establecer que el núcleo de aluminio no cambia su geometría durante el proceso de hidrólisis.

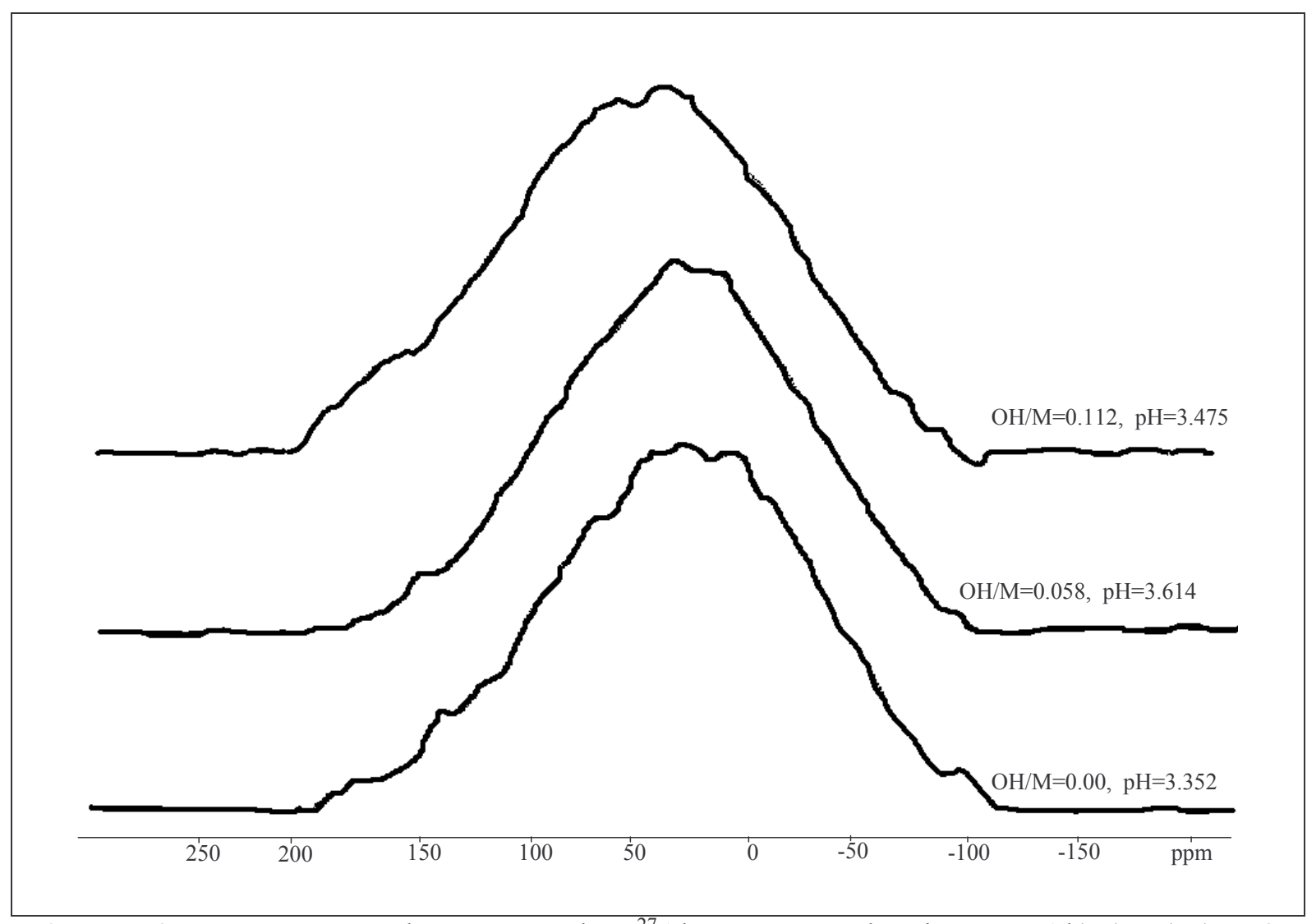

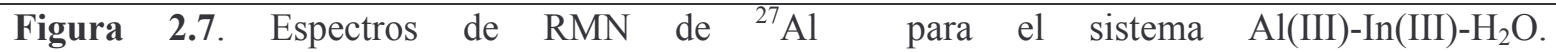
$[\mathrm{Al}(\mathrm{III})]=[\mathrm{In}(\mathrm{III})]=0.10 \mathrm{M}$

Por otro lado, los espectros de RMN de ${ }^{115}$ In muestran una señal muy ancha que no permite realizar un análisis de ella, ya que no es posible evidenciar cambios de geometría que indicarían la formación de especies polinucleares.

De esta forma los estudios de los núcleos de ${ }^{27} \mathrm{Al}$ y de ${ }^{115}$ In por medio de RMN no 
proporcionan información acerca de las posibles especies ternarias que se pudiesen formar en el sistema

\subsubsection{CONCLUSIONES}

Con base en los datos potenciométricos se establece que las curvas del sistema $\mathrm{Al}(\mathrm{III})-\mathrm{In}(\mathrm{III})-\mathrm{H}_{2} \mathrm{O}$ presentan un comportamiento característico, que al no ser igual al presentado por los sistemas binarios de $\mathrm{Al}(\mathrm{III})-\mathrm{H}_{2} \mathrm{O}$ y $-\mathrm{In}(\mathrm{III})-\mathrm{H}_{2} \mathrm{O}$ lleva a proponer la posibilidad de una interacción química entre los iones metálicos. Sin embargo, los refinamientos obtenidos por SUPERQUAD presentan una estadística similar que no nos permite establecer el mejor modelo químico. Al realizar estudios de RMN tanto para aluminio como para indio no fue posible, tampoco, obtener información que permita proponer de manera contundente un modelo químico, por lo que solamente se puede sugerir la posibilidad de formación de un complejo ternario de aluminio-indio-hidroxo $\left(\mathrm{AlIn}_{2}(\mathrm{OH})_{4}{ }^{5+}\right)$. Esto lleva a sugerir que este sistema en futuros estudios debe ser analizado, empleando técnicas experimentales de análisis químico adicionales a las empleadas en este trabajo para poder probar la existencia de las especies propuestas. 


\subsection{SISTEMA $\mathrm{Al}(\mathrm{III})-\mathrm{Ga}(\mathrm{III})-\mathrm{H}_{2} \mathrm{O}$}

\subsubsection{EXPERIMENTAL}

\subsubsection{Estudios Potenciométricos.}

Con la finalidad de identificar las especies y determinar las constantes de formación de estas se prepararon soluciones acuosas de $\mathrm{Al}(\mathrm{III}): \mathrm{Ga}(\mathrm{III})$ (con una relación molar de Al:Ga aproximadamente de 12:1) a partir de $\mathrm{Ga}\left(\mathrm{NO}_{3}\right)_{3} \mathrm{9H}_{2} \mathrm{O}$ (Baker Analyzed, qp) y de $\mathrm{Al}\left(\mathrm{NO}_{3}\right)_{3} 9 \mathrm{H}_{2} \mathrm{O}$ (Baker Analyzed, 99.1\%) (Tabla 2.3).

Tabla 2.3 Concentración de las soluciones acuosas de Al(III):Ga(III) empleadas para los estudios de Potenciometría

\begin{tabular}{cc}
\hline$[\mathrm{Al}(\mathrm{III})] / \mathrm{mol} \mathrm{L}^{-1}\left(10^{3}\right)$ & {$[\mathrm{Ga}(\mathrm{III})] / \mathrm{mol} \mathrm{L}^{-1}\left(10^{4}\right)$} \\
\hline 3.96 & 3.47 \\
7.92 & 6.94 \\
11.88 & 10.42 \\
15.84 & 13.89 \\
19.80 & 17.36 \\
\hline
\end{tabular}

Al menos dos muestras de cada solución fueron valoradas, registrando un mínimo de 70 puntos por valoración, con soluciones estandarizadas de hidróxido de sodio, libres de carbonatos Las variaciones de $\mathrm{pH}$ y potencial fueron registradas con un $\mathrm{pH}$-metro Radiometer-Tacussel LPH430T $(\Delta \mathrm{pH}=0.001)$, empleando un electrodo de vidrio combinado con referencia interna de $\mathrm{Ag} / \mathrm{AgCl}_{(\mathrm{s})}$. Se efectuó la calibración del equipo con soluciones buffer de referencia de $\mathrm{pH}=4.005 \mathrm{y} / \mathrm{o}$ 7.001. La temperatura de los sistemas se 
mantuvo constante a $25^{\circ} \mathrm{C}\left(\Delta \mathrm{T}=0.1^{\circ} \mathrm{C}\right)$ con un baño (VWR, modelo 12101-10) y empleando celdas de vidrio de doble camisa. Durante toda la experimentación se mantuvo un flujo constante de nitrógeno de alta pureza para evitar la carbonatación del sistema.

En todas las soluciones se empleo agua destilada y descarbonatada Tipo I (desionizador Millipore).

\subsubsection{Estudios de Conductimetría.}

Se efectuaron valoraciones conductimétricas de al menos dos muestras de soluciones acuosas de $\mathrm{Al}(\mathrm{III}): \mathrm{Ga}(\mathrm{III})$ (Tabla 2.4), preparadas a partir de $\mathrm{Al}\left(\mathrm{NO}_{3}\right)_{3} \bullet 9 \mathrm{H}_{2} \mathrm{O}$ (Sigma ACS, $99.1 \%$ ), $\mathrm{Ga}(\mathrm{III})\left(\mathrm{Ga}\left(\mathrm{NO}_{3}\right)_{3} \bullet 9 \mathrm{H}_{2} \mathrm{O}\right.$, Baker Analyzed, qp). Las valoraciones se realizaron con soluciones estandarizadas de hidróxido de sodio, registrando al menos 50 medidas de conductividad en cada caso. Se empleó un conductimétro Radiometer Copenhagen modelo CDM230 con una celda de cuatro polos de conductividad (cuatro anillos de platino platinizado) Radiometer Copehagen CDC865, monitoreando la temperatura in situ con un sensor de temperatura Radiometer Copenhagen modelo T201. La temperatura se mantuvo constante a $25^{\circ} \mathrm{C}\left(\Delta \mathrm{T}=0.1^{\circ} \mathrm{C}\right)$ con un baño Polystat modelo 12101 10. Durante la experimentación se mantuvo un flujo constante de nitrógeno para evitar la carbonatación del sistema. 
Tabla 2.4. Concentración de las soluciones acuosas de Al(III):Ga(III) empleadas para los estudios de Conductimetría

\begin{tabular}{cc}
\hline$[\mathrm{Al}(\mathrm{III})] / \mathrm{mol} \mathrm{L}^{-1}\left(10^{3}\right)$ & {$[\mathrm{Ga}(\mathrm{III})] / \mathrm{mol} \mathrm{L}^{-1}\left(10^{4}\right)$} \\
\hline 5.65 & 3.20 \\
11.29 & 6.40 \\
16.94 & 9.60 \\
22.58 & 12.8 \\
28.23 & 16.0 \\
\hline
\end{tabular}

\subsubsection{Estudios de RMN.}

Soluciones acuosas de Al(III) 0.12M:Ga(III) 0.01M fueron preparadas a partir de $\mathrm{Al}\left(\mathrm{NO}_{3}\right)_{3} \bullet 9 \mathrm{H}_{2} \mathrm{O}($ Sigma ACS, $99.1 \%), \mathrm{Ga}(\mathrm{III})\left(\mathrm{Ga}\left(\mathrm{NO}_{3}\right)_{3} \cdot 9 \mathrm{H}_{2} \mathrm{O}\right.$, Baker Analyzed, qp). Se hidrolizaron dichas soluciones hasta alcanzar la adecuada relación $\mathrm{OH} / M(0.0 \leq \mathrm{OH} / M \leq 2.5)$ mediante una lenta adición $(0.5 \mathrm{~mL} / \mathrm{min})$ de solución estándar de hidróxido de sodio, con agitación vigorosa y calentamiento. El pH fue registrado para cada solución hidrolizada.

Los espectros de RMN de ${ }^{71} \mathrm{Ga}{ }^{27} \mathrm{Al}$ fueron obtenidos en un espectrómetro Bruker DMX500 a una temperatura de $25.0{ }^{\mathrm{O}} \mathrm{C} . \mathrm{Ga}\left(\mathrm{H}_{2} \mathrm{O}\right)_{6}{ }^{3+}$ y $\mathrm{Al}\left(\mathrm{H}_{2} \mathrm{O}\right)_{6}{ }^{3+}$ fueron empleadas como referencias de desplazamiento químico respectivamente y $\mathrm{D}_{2} \mathrm{O}$ se utilizó para fijar la señal de campo externo. La excitación empleada para el caso de ${ }^{27} \mathrm{Al}$ fue de $130.32 \mathrm{MHz}$, mientras que para ${ }^{71}$ Ga se empleó una excitación de $152.53 \mathrm{MHz}$. 


\subsubsection{RESULTADOS Y DISCUSIÓN}

\subsubsection{Estudios Potenciométricos}

Las curvas características para la valoración del sistema $\mathrm{Al}(\mathrm{III})-\mathrm{Ga}(\mathrm{III})-\mathrm{H}_{2} \mathrm{O}$ se muestran en la Figura 2.8, donde se puede apreciar que existe un incremento notable del pH al inicio de la valoración, seguido de un ligero incremento de éste conforme la valoración va progresando, esto es hasta alcanzar $\mathrm{OH} / \mathrm{M}=2.5$, ya que al igual que en el sistema de $\mathrm{Al}(\mathrm{III})-\mathrm{H}_{2} \mathrm{O}$ y de $\mathrm{Ga}(\mathrm{III})-\mathrm{H}_{2} \mathrm{O}$ [Capítulo 1] por encima de esta relación tiene lugar la precipitación en el sistema.

Los datos obtenidos en estas valoraciones fueron alimentados al programa SUPERQUAD para determinar las posibles estequiometrías y obtener el refinamiento de las constantes de las especies que se forman durante el proceso de hidrólisis del sistema Al(III)-Ga(III)- $\mathrm{H}_{2} \mathrm{O}$. Para ello se consideraron tanto a las especies de Al(III), Ga(III) (mostrados anteriormente en el estudio de sistemas binarios, Tablas 1.5 y 1.11) así como posibles complejos ternarios, de acuerdo a la ecuación 2.3.

$$
\begin{gathered}
\mathrm{iAl}^{3+}+\mathrm{jGa}^{3+}+\mathrm{kH}_{2} \mathrm{O}=\mathrm{Al}_{\mathrm{i}} \mathrm{Ga}_{\mathrm{j}}(\mathrm{OH})_{\mathrm{k}}^{3(\mathrm{i}+\mathrm{j})-\mathrm{k}}+\mathrm{kH}^{+} \\
* \beta_{\mathrm{ijk}}=\frac{\left[\mathrm{Al}_{\mathrm{i}} \mathrm{Ga}_{\mathrm{j}}(\mathrm{OH})_{\mathrm{k}}^{3(\mathrm{i}+\mathrm{j})-\mathrm{k}}\right]\left[\mathrm{H}^{+}\right]^{\mathrm{k}}}{\left[\mathrm{Al}^{3+}\right]^{\mathrm{i}}\left[\mathrm{Ga}^{3+}\right]^{\mathrm{j}}}
\end{gathered}
$$




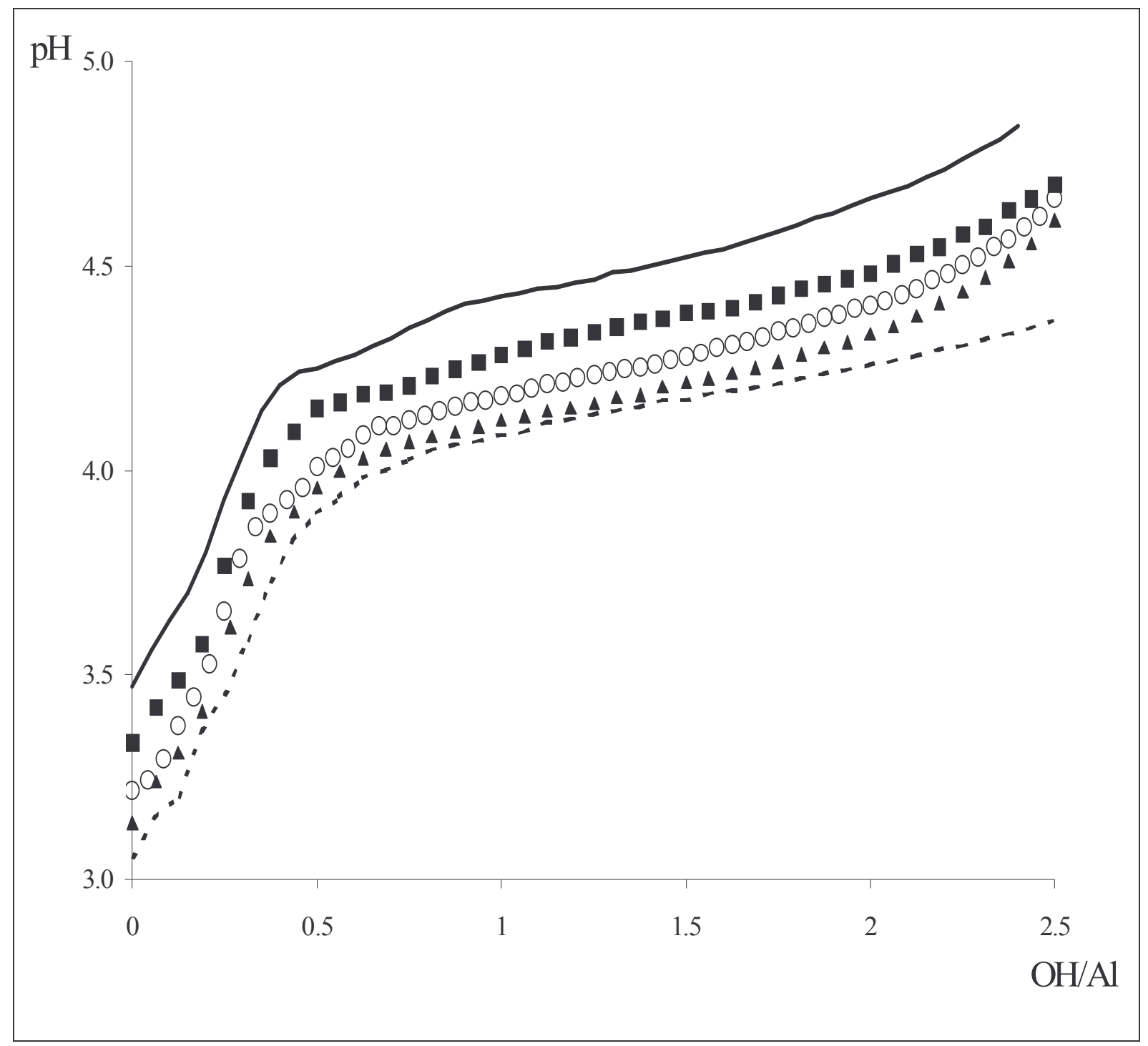

Figura 2.8. Curvas de valoración para el sistema $\mathrm{Al}(\mathrm{III})-\mathrm{Ga}(\mathrm{III})-\mathrm{H}_{2} \mathrm{O}$ a $25^{\circ} \mathrm{C}(-) 3.96\left(10^{-}\right.$

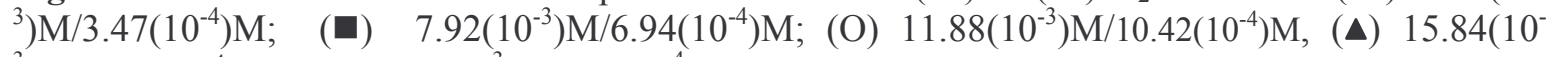
$\left.{ }^{3}\right) \mathrm{M} / 13.89\left(10^{-4}\right) \mathrm{M},(--) 19.80\left(10^{-3}\right) / 17.36\left(10^{-4}\right) \mathrm{M}$.

El mejor refinamiento que se obtuvo se muestra en la Tabla 2.5, obteniendo un modelo que considera la formación de la especie $\mathrm{Al}_{12} \mathrm{Ga}(\mathrm{OH})_{28}{ }^{11+}$. Es importante destacar que en este modelo no aparecen las especies $\mathrm{Al}(\mathrm{OH})_{3}$ y $\mathrm{Al}_{3}(\mathrm{OH})_{4}{ }^{5+}$ determinadas para el sistema $\mathrm{Al}(\mathrm{III})-\mathrm{H} 2 \mathrm{O}^{27,} 42,48,49$, lo cual implica que probablemente estas especies no se 
forman, posiblemente por la formación del complejo mixto de $\mathrm{Al}_{12} \mathrm{Ga}(\mathrm{OH})_{28}{ }^{1{ }^{1+}}$. Por otro lado, las constantes de equilibrio determinadas para este modelo son consistentes con aquéllas obtenidas para sistemas de $\mathrm{Al}(\mathrm{III})-\mathrm{H}_{2} \mathrm{O}$ (Tabla 1.5) y $\mathrm{Ga}(\mathrm{III})-\mathrm{H}_{2} \mathrm{O}$ (Tabla 1.11) obtenidas en este trabajo.

Tabla 2.5 Valores de las constantes de formación para el mejor refinamiento determinado por el programa SUPERQUAD a partir de información potenciométrica del sistema $\mathrm{Al}(\mathrm{III})-\mathrm{Ga}(\mathrm{III})-\mathrm{H}_{2} \mathrm{O}\left(\mathrm{T}=25^{\circ} \mathrm{C}\right)$

\begin{tabular}{ccc}
\hline Especies & $-\log { }^{*} \beta$ & Parámetros $^{\mathrm{a}}$ \\
\hline $\mathrm{Al}(\mathrm{OH})^{2+}$ & $5.35 \pm 0.13$ & Estadísticos $^{2+}$ \\
$\mathrm{Al}_{13}(\mathrm{OH})_{32^{7+}}$ & $110.46 \pm 0.14$ & $\sigma^{\mathrm{c}}=0.7252$ \\
$\mathrm{Ga}(\mathrm{OH})_{2}{ }^{+}$ & $7.13^{\mathrm{b}}$ & $\mathrm{U}^{\mathrm{d}}=240.87$ \\
$\mathrm{Ga}(\mathrm{OH})_{3}$ & $10.62^{\mathrm{b}}$ & $\left(\chi^{2}\right)^{\mathrm{e}}=37.10$ \\
$\mathrm{Al}_{12} \mathrm{Ga}(\mathrm{OH})_{28}{ }^{1{ }^{+}}$ & $88.66 \pm 0.15$ &
\end{tabular}

${ }^{a} 470$ datos se alimentaron a SUPERQUAD. ${ }^{b}$ valor constante durante el refinamiento. ${ }^{c}$ desviación estándar total para el refinamiento ${ }^{\mathrm{d}}$ Suma de los residuales al cuadrados del potencial de celda. ${ }^{\mathrm{e}} \mathrm{Chi}$-cuadrada para el refinamiento

Por otro lado, se determinaron las curvas de la función de formación $\mathrm{Z}$ en función del pH (Figura 2.9), determinadas de acuerdo a la ecuación 2.4 a partir de los datos potenciométricos. Se puede observar que la función $\mathrm{Z}$ depende de la $[M]_{\text {total }}\left([\mathrm{Al}(\mathrm{III})]_{\mathrm{total}}+\right.$ $\left.[\mathrm{Ga}(\mathrm{III})]_{\text {total }}\right)$, ya que conforme ésta se incrementa las curvas tienen un desplazamiento en la escala de $\mathrm{pH}$. Este comportamiento es característico para sistemas en los cuales especies polinucleares están presentes, como lo explican Baes y Mesmer $^{1}$ y Hartley y cols ${ }^{2}$, lo que resulta consistente con la formación de las especies polinucleares de $\mathrm{Al}_{13}(\mathrm{OH})_{32}{ }^{7+} \mathrm{y}$ $\mathrm{Al}_{12} \mathrm{Ga}(\mathrm{OH})_{28}{ }^{11+}$ propuestas en este trabajo (Tablas 1.5 y 2.3 ). 


$$
\mathrm{Z}=\left[\frac{\left[\mathrm{OH}^{-}\right]_{\text {total }}-\left[\mathrm{K}_{\mathrm{w}} / 10^{-\mathrm{pH}}\right]+\left[10^{-\mathrm{pH}}\right]}{[\mathrm{Ga}(\mathrm{III})]_{\text {total }}+[\mathrm{Al}(\mathrm{III})]_{\text {total }}}\right]
$$

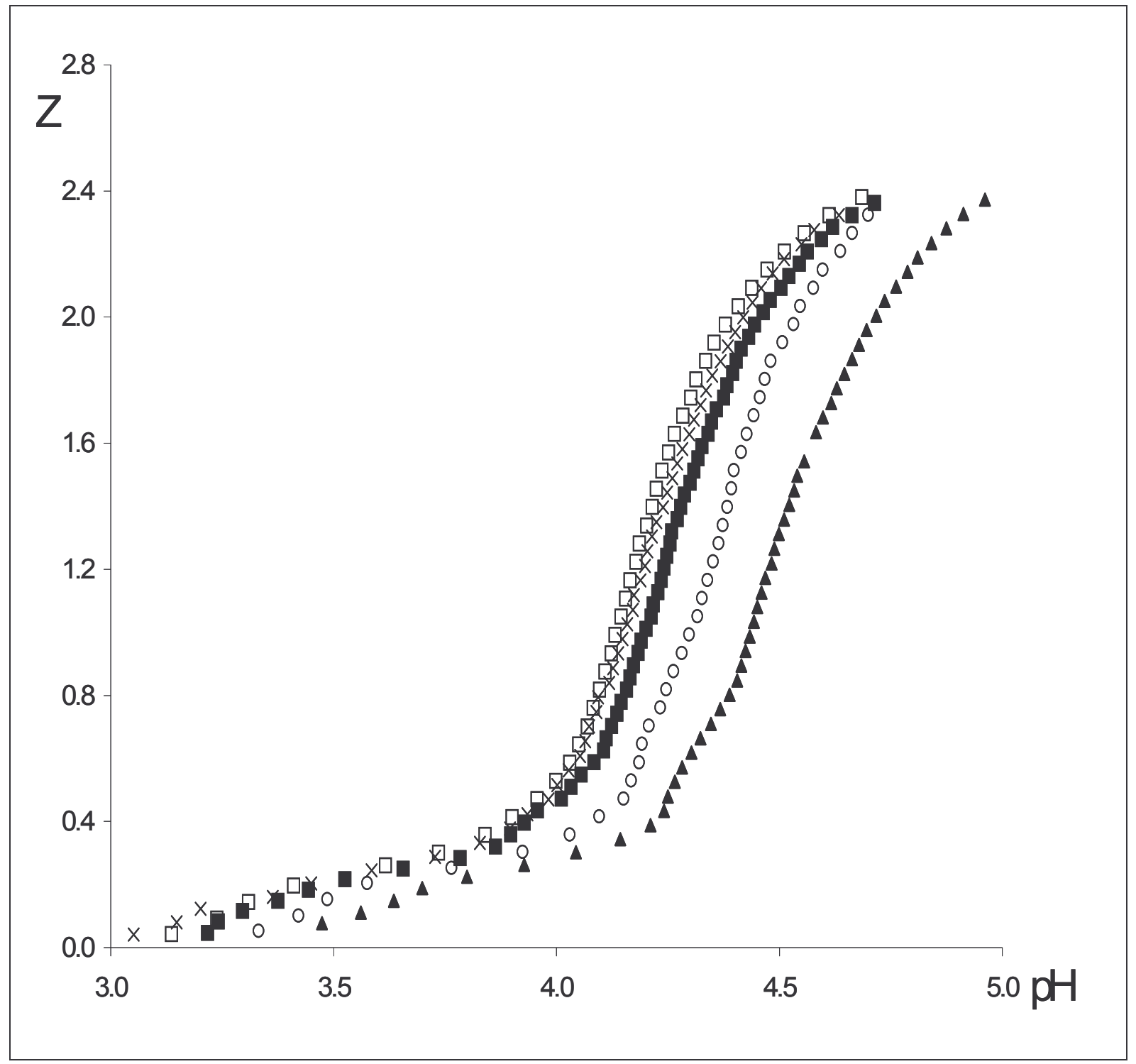

Figura 2.9. Gráfico de $\mathrm{Z}$ como función del $\mathrm{pH}$ para el sistema de $\mathrm{Al}(\mathrm{III})-\mathrm{Ga}(\mathrm{III})-\mathrm{H}_{2} \mathrm{O}$, para diferentes concentraciones de $[\mathrm{Al}(\mathrm{III})]_{\mathrm{t}} /$ / [Ga(III) $]_{\mathrm{t}} .:(\mathbf{\Delta}) 3.96\left(10^{-3}\right) \mathrm{M} / 3.47\left(10^{-4}\right) \mathrm{M}$; (O) $7.92\left(10^{-}\right.$ $\left.{ }^{3}\right) \mathrm{M} / 6.94\left(10^{-4}\right) \mathrm{M}$; (匹) $11.88\left(10^{-3}\right) \mathrm{M} / 10.42\left(10^{-4}\right) \mathrm{M}$ ，(X) $15.84\left(10^{-3}\right) \mathrm{M} / 13.89\left(10^{-4}\right) \mathrm{M}$ ，(口) $19.80\left(10^{-}\right.$ $\left.{ }^{3}\right) / 17.36\left(10^{-4}\right) \mathrm{M}$. 


\subsubsection{Estudios Conductimétricos}

Las valoraciones conductimétricas características para el sistema Al(III)-Ga(III)$\mathrm{H}_{2} \mathrm{O}$ en un intervalo de concentraciones de $6.32 \mathrm{E}-3 \mathrm{M} \leq[M] \leq 2.96 \mathrm{E}-2 \mathrm{M}$ se observan en la Figura 2.10, donde se puede apreciar que al inicio de la valoración $(\mathrm{OH} / M \leq 0.3)$ se tiene una ligera disminución de la conductividad, posteriormente ésta permanece prácticamente constante hasta que se alcanza una relación de $\mathrm{OH} / M=2.5$. Por otro lado, cuando se tiene una concentración de $[M]=0.13 \mathrm{M}$ se observa una disminución de la conductividad hasta alcanzar una relación $\mathrm{OH} / M=0.7$ y después se observa un incremento en ella conforme la hidrólisis del sistema progresa. Las valoraciones se realizaron hasta antes de que tenga lugar la precipitación (es decir $\mathrm{OH} / M \leq 2.5$ ).

Con la finalidad de validar el modelo químico propuesto en este trabajo para el sistema ternario $\mathrm{Al}(\mathrm{III})-\mathrm{Ga}(\mathrm{III})-\mathrm{H} 2 \mathrm{O}$ (Tabla 2.5) se realizó el ajuste gráfico de las conductividades obtenidas experimentalmente y las estimadas con base en la ecuación 2.5. Las fracciones molares de las especies de $\mathrm{Al}(\mathrm{III}), \mathrm{Ga}(\mathrm{III})$ y el complejo ternario se calcularon con ayuda del programa computacional MEDUSA ${ }^{22}$. Para el caso del complejo ternario la fracción molar puede ser considerada ya sea con base en el diagrama de fracción molar de $\mathrm{Al}(\mathrm{III})$ o bien a partir del diagrama de $\mathrm{Ga}(\mathrm{III})$. La concentración de $\mathrm{Na}^{+}$se determinó a partir de la cantidad de hidróxido de sodio adicionado al sistema, mientras que la concentración de $\mathrm{NO}_{3}{ }^{-}$se estimo en base a las cantidades de $\mathrm{Al}\left(\mathrm{NO}_{3}\right)_{3}$ y $\mathrm{Ga}\left(\mathrm{NO}_{3}\right)_{3}$, Las concentraciones de $\mathrm{H}^{+}$y $\mathrm{OH}^{-}$se calcularon con base en el $\mathrm{pH}$ experimental del sistema. 


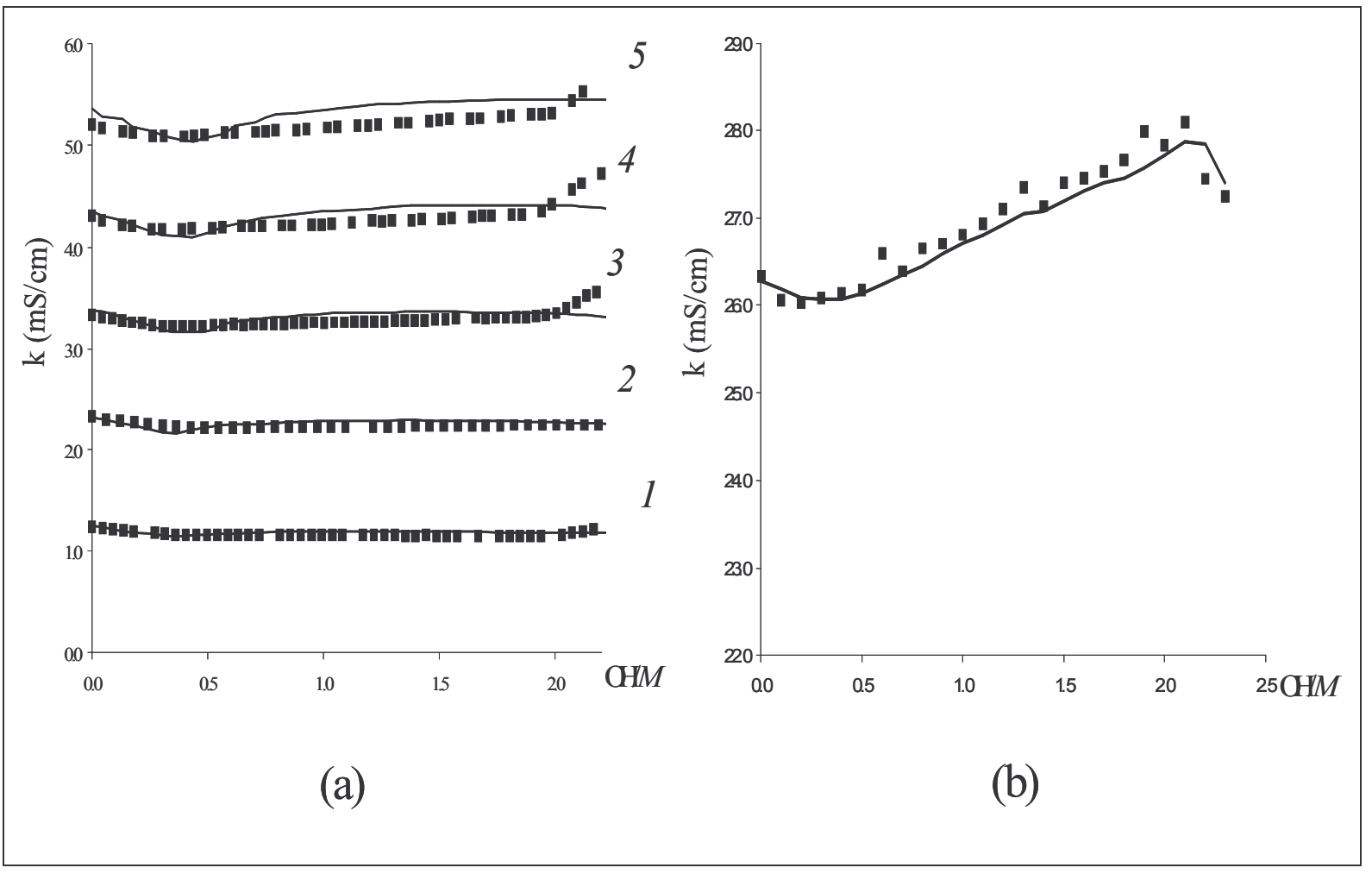

Figura 2.10. Valoraciones conductimétricas para el sistema $\mathrm{Al}(\mathrm{III})-\mathrm{Ga}(\mathrm{III})-\mathrm{H}_{2} \mathrm{O}$.

(a). (1) $\left.[M]=6.32\left(10^{-3}\right) \mathrm{M},(2)[M]=12.1410^{-3}\right) \mathrm{M},(3)[M]=17.96\left(10^{-3}\right) \mathrm{M},(4)[M]=23.78\left(10^{-3}\right) \mathrm{M},(5)$ $[M]=9.6\left(10^{-3}\right) \mathrm{M} ;(\mathrm{b}) .[M]=0.13 \mathrm{M}$ Las líneas representan la conductividad estimada empleando el modelo obtenido en la Tabla 2.3 por medio del programa SUPERQUAD ${ }^{6-7}$. Los marcadores representan los valores de conductividad experimentales $([M]=[\mathrm{Al}(\mathrm{III})]+[\mathrm{Ga}(\mathrm{III})])$

Para el ajuste se estimaron mediante iteración, los coeficientes de conductividad equivalente $(\lambda)$ para todas las especies. En la Tabla 2.6 se presentan los valores de los coeficientes de conductividad equivalente estimados. Para el caso de $\mathrm{H}^{+}$y $\mathrm{OH}^{-}$ corresponden a los reportados en la literatura ${ }^{50}$, mientras que los coeficientes de las conductividades equivalentes $(\lambda)$ de las especies de $\mathrm{Al}(\mathrm{III})$ y $\mathrm{Ga}(\mathrm{III})$ corresponden con los valores determinados en los sistemas binarios estudiados en el Capitulo I (Tablas 1.6 y 1.13), lo cual corrobora los estudios antes realizados. Es importante puntualizar, que de 
igual manera que en los sistemas binarios, los valores de estos coeficientes se incrementaron conforme la concentración total de los cationes disminuía en el sistema, lo cual como ya se había mencionado es un comportamiento esperado, ya que dicho parámetro está relacionado con la movilidad de los iones, la cual alcanza valores límite a dilución infinita $^{50}$.

$$
\begin{aligned}
& \kappa=|z| \lambda_{\mathrm{Na}^{+}}\left[\mathrm{Na}^{+}\right]+|z| \lambda_{\mathrm{NO}^{-}}\left[\mathrm{NO}_{3}^{-}\right]+|z| \lambda_{\mathrm{H}^{+}}\left[\mathrm{H}^{+}\right]+\mid z \lambda_{\mathrm{OH}^{-}}[\mathrm{OH}]+ \\
& \sum|\mathrm{z}| \lambda_{\mathrm{Al}_{\mathrm{i}}(\mathrm{OH})_{\mathrm{k}}^{3 i-\mathrm{k}}} \mathrm{F}_{\mathrm{Al}_{\mathrm{i}}\left(\mathrm{OH}_{\mathrm{k}}{ }^{3 i-\mathrm{k}}\right.}[\mathrm{Al}(\mathrm{III})]_{\text {total }}+ \\
& \sum|\mathrm{z}| \lambda_{\mathrm{Ga}_{i}(\mathrm{OH})_{\mathrm{k}}^{3 i-k}} \mathrm{~F}_{\mathrm{Ga}_{i}(\mathrm{OH})_{\mathrm{k}}^{3 i-k}}[\mathrm{Ga}(\mathrm{III})]_{\text {total }}+ \\
& \sum \mathrm{z} \mid \lambda_{\mathrm{Al}_{\mathrm{i}} \mathrm{Ga}_{\mathrm{j}}(\mathrm{OH})_{\mathrm{k}}^{3(+\mathrm{i})-\mathrm{k}}} \mathrm{F}_{\mathrm{Al}_{\mathrm{i}} \mathrm{Ga}_{\mathrm{j}}(\mathrm{OH})_{\mathrm{k}}^{3(\mathrm{i}+\mathrm{j})-\mathrm{k}}}[\mathrm{Al}(\mathrm{III})]_{\mathrm{total}}
\end{aligned}
$$

Tabla 2.6. Valores estimados de Conductividad equivalente para el ajuste de las curvas de valoración conductimétricas para el sistema $\mathrm{Al}(\mathrm{III})-\mathrm{Ga}(\mathrm{III})-\mathrm{H}_{2} \mathrm{O}$.

\begin{tabular}{lcc}
\hline Sistema & Especies & $\lambda / \mathrm{mS} \mathrm{cm}^{2}$ equivalente $^{-1}$ \\
\hline & $\mathrm{Al}^{3+}$ & $4-14.8$ \\
$\mathrm{Ga}^{3+}$ & $30-50$ \\
$\mathrm{Ga}(\mathrm{OH})_{2}^{+}$ & $15-20$ \\
$\mathrm{Ga}(\mathrm{OH})_{3}$ & 0 \\
$\mathrm{Al}(\mathrm{III})-\mathrm{Ga}(\mathrm{III})-\mathrm{H}_{2} \mathrm{O}$ & $\mathrm{Al}(\mathrm{OH})^{2+}$ & 10 \\
& $\mathrm{Al}{ }_{13}(\mathrm{OH})_{32}{ }^{7+}$ & $37-43$ \\
& $\mathrm{Al}_{12} \mathrm{Ga}(\mathrm{OH})_{28}{ }^{11+}$ & $47-50$ \\
& $\mathrm{Na}^{+}$ & $5.5-14$ \\
$\mathrm{NO}^{3-}$ & $48-68$ \\
\hline
\end{tabular}

En la Figura 2.10 se muestra que existe una adecuada sobreposición entre las conductividades experimentales y las estimadas con el modelo de SUPERQUAD, lo cual nos permite proponer la validez del modelo propuesto en la Tabla 2.5. 


\subsubsection{Estudios de RMN}

En los experimentos de $\mathrm{RMN}$ para el sistema $\mathrm{Al}(\mathrm{III})-\mathrm{Ga}(\mathrm{III})-\mathrm{H}_{2} \mathrm{O}$ se determinaron tanto los espectros de ${ }^{71} \mathrm{Ga}$ y de ${ }^{27} \mathrm{Al}$. En la Figura 2.11 se muestran los espectros de RMN de ${ }^{71} \mathrm{Ga}$ - para distintos grados de hidrólisis. Se puede observar que para una relación de $\mathrm{OH} / M=0.0(\mathrm{pH}=2.71)$ solamente se observa un pico en $0 \mathrm{ppm}$ relacionado a la resonancia de un núcleo octaédrico de $\mathrm{Ga}(\mathrm{III})$ que corresponde a la especie $\mathrm{Ga}\left(\mathrm{H}_{2} \mathrm{O}\right)_{6}{ }^{3+}$. De igual manera que para el sistema binario $\mathrm{Ga}(\mathrm{III})-\mathrm{H}_{2} \mathrm{O}$, el cual ya había sido estudiado anteriormente $^{48}$, conforme la hidrólisis del sistema se incrementa hasta una relación de $\mathrm{OH} / M=0.20(\mathrm{pH}=2.995)$ el pico se va ensanchando y su intensidad disminuye, lo cual puede indicar un ambiente químico más distorsionado alrededor de los núcleos de Ga y/o la

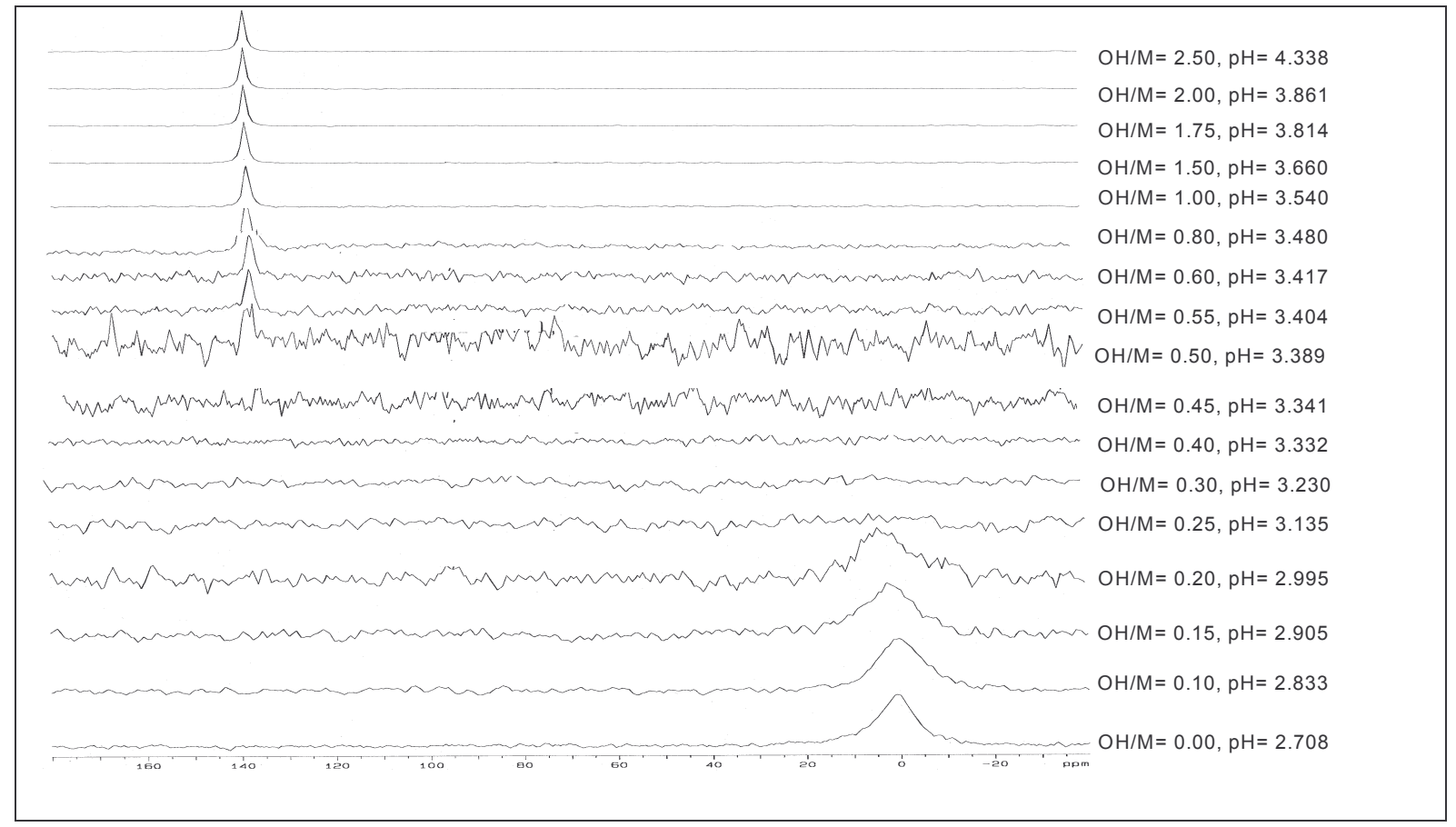

Figura 2.11. Espectros de $\mathrm{RMN}$ para ${ }^{71} \mathrm{Ga}$ para el sistema $\mathrm{Al}(\mathrm{III})-\mathrm{Ga}(\mathrm{III})-\mathrm{H}_{2} \mathrm{O}$ con diferentes grados de hidrólisis $\left([\mathrm{Al}(\mathrm{III})]_{\text {total }}=0.12 \mathrm{M}:[\mathrm{Ga}(\mathrm{III})]_{\text {total }}=0.01 \mathrm{M}\right)$ 
presencia de otras especies químicas de la misma geometría. En este caso se atribuye a la aparición de la especie $\mathrm{Ga}(\mathrm{OH})_{2}{ }^{+}$. En un intervalo de $0.25 \leq \mathrm{OH} / M \leq 0.45$ (c), la señal desaparece lo cual indica la formación y predominio de los monómeros de Ga $(1,2)$ y $(1,3)$, lo cual coincide con el diagrama de distribución (Figura 2.12) determinado a partir de los datos de la Tabla 2.5. A partir de una relación $\mathrm{OH} / M=0.50(\mathrm{pH}=3.389)$ aparece un pico estrecho en un desplazamiento químico de 138 ppm, la posición de este pico corresponde con lo reportado por Bradley y colaboradores ${ }^{64}$ y Thomas y colaboradores $^{65}$ indicando la presencia de un núcleo de galio tetraédricamente coordinado, lo cual corresponde, de acuerdo al diagrama de distribución (Figura 2.12), a la aparición de la especie $\mathrm{Al}_{12} \mathrm{Ga}(\mathrm{OH})_{28}{ }^{11+}$ y que puede sugerirse por tanto un núcleo de galio tetraédrico rodeado de 12 átomos de aluminio octaédrico $\left(\mathrm{Ga}_{\mathrm{Td}} \mathrm{Al}_{12}\right)$ teniendo la configuración de un ion tipo Keggin (Figura 2.13). Conforme la hidrólisis progresa hasta $\mathrm{OH} / M \leq 2.5 \quad(\mathrm{pH}=4.34)$ la intensidad de esta señal se incrementa así como su estrechez, lo cual indica un ambiente poco distorsionado y/o que esta señal se debe a una sola especie. Lo anterior confirma que dicha señal se debe al complejo ternario de $\mathrm{Al}_{12} \mathrm{Ga}(\mathrm{OH})_{28}{ }^{11+}$, ya que como se observa en el diagrama de distribución la fracción molar de esta se incrementa al aumentar el pH, llegando a predominar en el $4.0 \leq \mathrm{pH}$. 


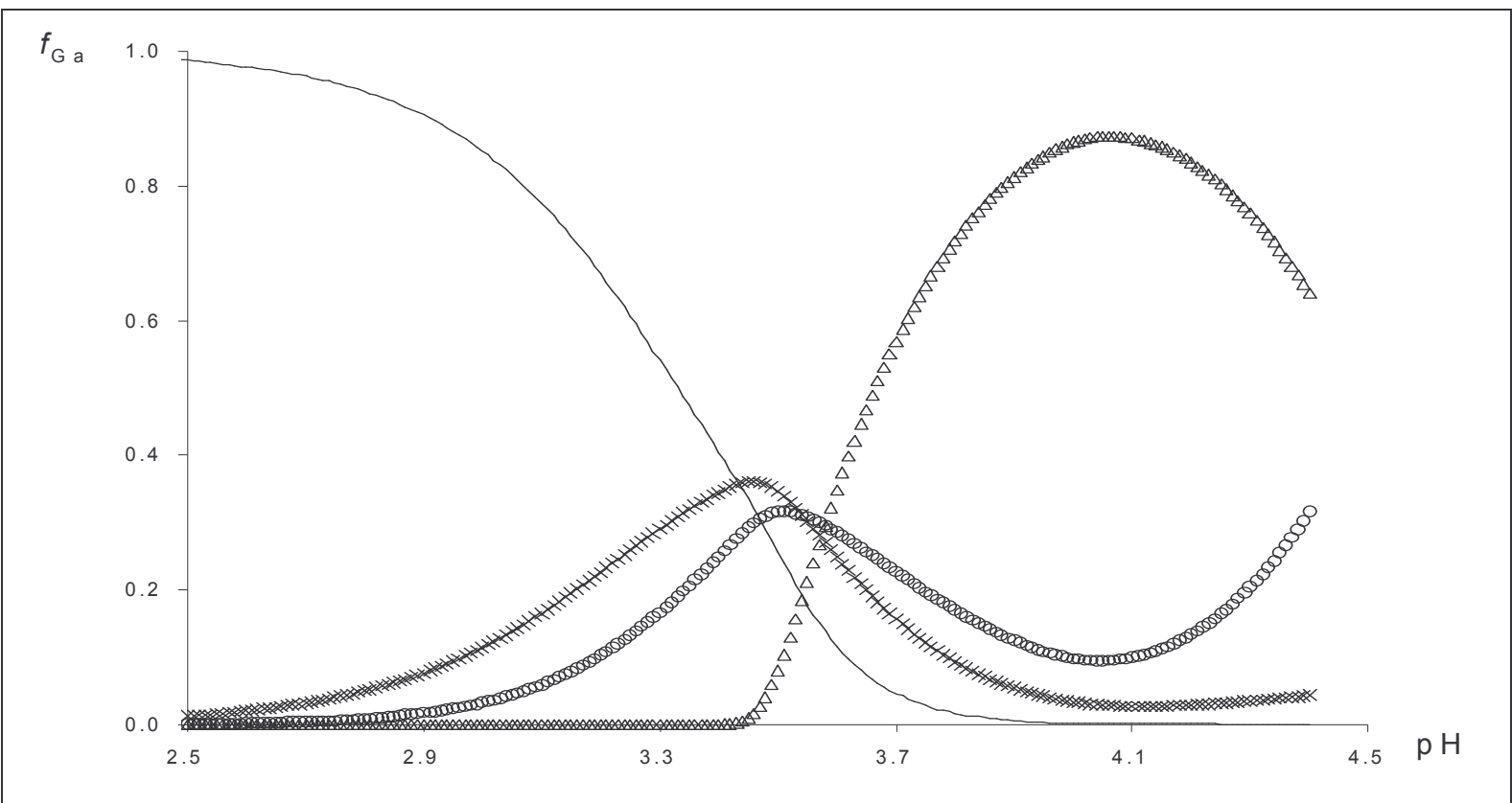

Figura 2.12. Diagrama de Distribución de Fracciones molares de especie, para especies de Ga(III) con base en el modelo propuesto en la Tabla 2.5 en el sistema $\mathrm{Al}(\mathrm{III})-\mathrm{Ga}(\mathrm{III})-\mathrm{H}_{2} \mathrm{O}$. (-) $\mathrm{Ga}^{3+}$, (X) $\mathrm{Ga}(\mathrm{OH})_{2}{ }^{+},(\mathrm{O}) \mathrm{Ga}(\mathrm{OH})_{3},(\Delta) \mathrm{Al}_{12} \mathrm{Ga}(\mathrm{OH})_{28}{ }^{11+}$.

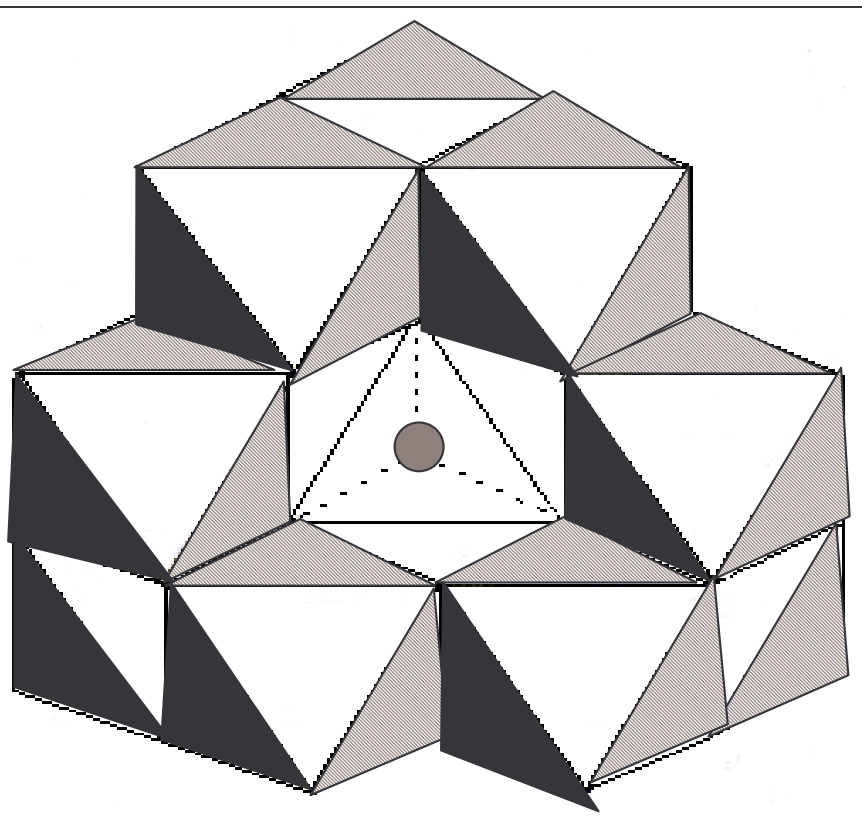

Figura 2.13. Estructura de un ion tipo Keggin, en el que el átomo central posee una geometría tetraédrica y se encuentra rodeado por doce átomos octaédricamente coordinados 
La realización de un estudio cuantitativo a partir de la información de las señales de RMN para ${ }^{71} \mathrm{Ga}$ no fue posible debido a que la señal obtenida en 0ppm es muy ancha lo cual dificultaría la deconvolución de la señal, y si esto se realizará el error asociado al procedimiento sería relativamente alto. Por otro lado, en el intervalo de $3.135 \leq \mathrm{pH} \leq 3.341$ no se observa señal alguna por lo que no sería posible obtener una fracción de área. Sin embargo, consideramos que a partir de la comparación de las señales de RMN con el diagrama de distribución de fracciones molares de especie es posible poder evidenciar la presencia del complejo ternario polinuclear $\mathrm{Al}_{12} \mathrm{Ga}(\mathrm{OH})_{28}{ }^{11+}$ y establecer de esta manera que el modelo propuesto en la Tabla 2.5 explica adecuadamente el comportamiento de las señales de ${ }^{71} \mathrm{Ga}$ obtenidas por RMN.

Por otro lado, en la Figura 2.14 se muestran los espectros de RMN para ${ }^{27} \mathrm{Al}$ a diferentes grados de hidrólisis del sistema $\mathrm{Al}(\mathrm{III})-\mathrm{Ga}(\mathrm{III})-\mathrm{H}_{2} \mathrm{O}$. Es posible observar un solo pico en $0 \mathrm{ppm}$ en el intervalo de $0 \leq \mathrm{OH} / \mathrm{M} \leq 1.50$, es decir $2.71 \leq \mathrm{pH} \leq 3.66$, lo que permite establecer el predominio de especies de Al(III) que presentan geometría octaédrica. De acuerdo con el diagrama de distribución (Figura 2.15), en dicho intervalo de $\mathrm{pH}$ predomina la especie $\mathrm{Al}^{3+}$ y la presencia de una pequeña fracción de la especie $\mathrm{Al}(\mathrm{OH})^{2+}$. Por otro lado, en la relación $\mathrm{OH} / M=2.0(\mathrm{pH}=3.861)$, se observa la aparición de una señal en 63 ppm que de acuerdo al diagrama de distribución de especies correspondería a la aparición de la especie $\mathrm{Al}_{12} \mathrm{Ga}(\mathrm{OH})_{28}{ }^{11+}$. Conforme el $\mathrm{pH}$ se incrementa, y por tanto el grado de hidrólisis, la intensidad de la señal aumenta, de manera tal que en aproximadamente $\mathrm{pH}=4.338$ la intensidad de esta señal es mayor que la del pico observado en $0 \mathrm{ppm}$. De acuerdo al diagrama de distribución de especies corresponde al pH en el cual la fracción molar de 
$\mathrm{Al}_{12} \mathrm{Ga}(\mathrm{OH})_{28}{ }^{11+}$ es mayor que la del hexaacuocomplejo de aluminio $\left(\mathrm{Al}^{3+}\right.$ o $\left.\mathrm{Al}\left(\mathrm{H}_{2} \mathrm{O}\right)_{6}{ }^{3+}\right)$. Lo anterior lleva a sugerir que la señal observada en 63 ppm se debe a los núcleos de aluminio octaédricamente coordinados en la especie $\mathrm{Al}_{12} \mathrm{Ga}(\mathrm{OH})_{28}{ }^{11+}$ (de acuerdo a una estructura tipo Keggin) o bien que es resultado del promedio de los núcleos de aluminio octaédricos tanto en el complejo ternario como en la especie $\mathrm{Al}_{13}(\mathrm{OH})_{32}{ }^{7+}$ y del aluminio tetraédricamente coordinado en esta última. Esto contradice a lo que ha sido sugerido por Akitt y colaboradores ${ }^{42}$, que la atribuye únicamente al núcleo de aluminio tetraédrico dentro de la estructura de la especie $\mathrm{Al}_{13}(\mathrm{OH})_{32}{ }^{7+}$.

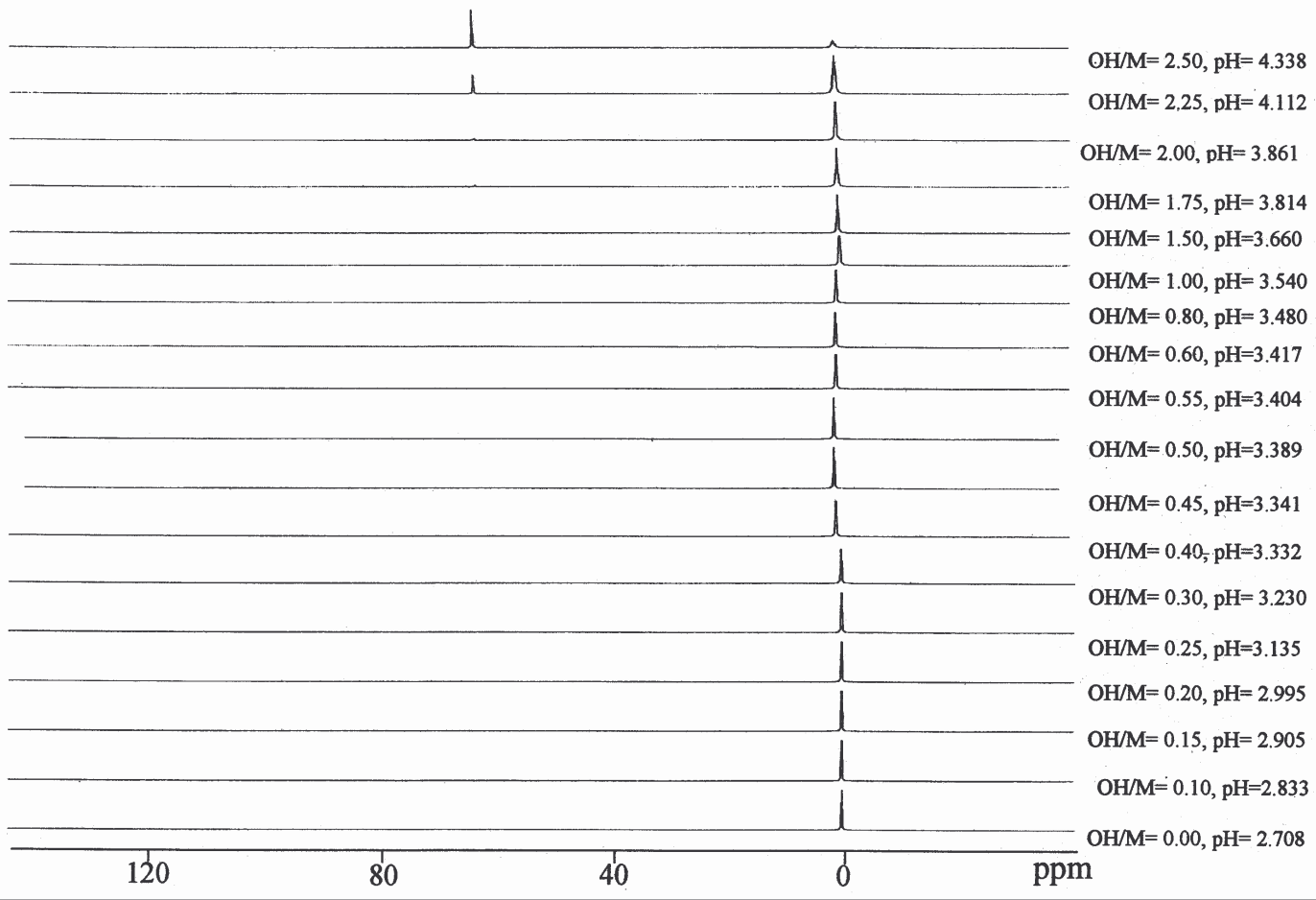

Figura 2.14. Espectros de $\mathrm{RMN}$ para ${ }^{27} \mathrm{Al}$ para el sistema $\mathrm{Al}(\mathrm{III})-\mathrm{Ga}(\mathrm{III})-\mathrm{H}_{2} \mathrm{O}$ con diferentes grados de hidrólisis ([Al(III) $\left.]_{\text {total }}=0.12 \mathrm{M}:[\mathrm{Ga}(\mathrm{III})]_{\mathrm{total}}=0.01 \mathrm{M}\right)$ 
Es importante destacar que en los espectros de ${ }^{27} \mathrm{Al}-\mathrm{RMN}$ para el sistema ternario no se observa señal alguna en 5 ppm, la cual se había caracterizado en el sistema binario de $\mathrm{Al}(\mathrm{III})-\mathrm{H}_{2} \mathrm{O}$ y que se había atribuido a una especie polinuclear de bajo peso molecular $\left(\mathrm{Al}_{3}(\mathrm{OH})_{4}{ }^{5+}\right)$. La desaparición de esta señal da evidencia de que dicha especie no sé esta formando en el sistema, como se propuso en los estudios potenciométrico y conductimétrico, debido a la formación del complejo $\mathrm{Al}_{12} \mathrm{Ga}(\mathrm{OH})_{28}{ }^{11+}$.

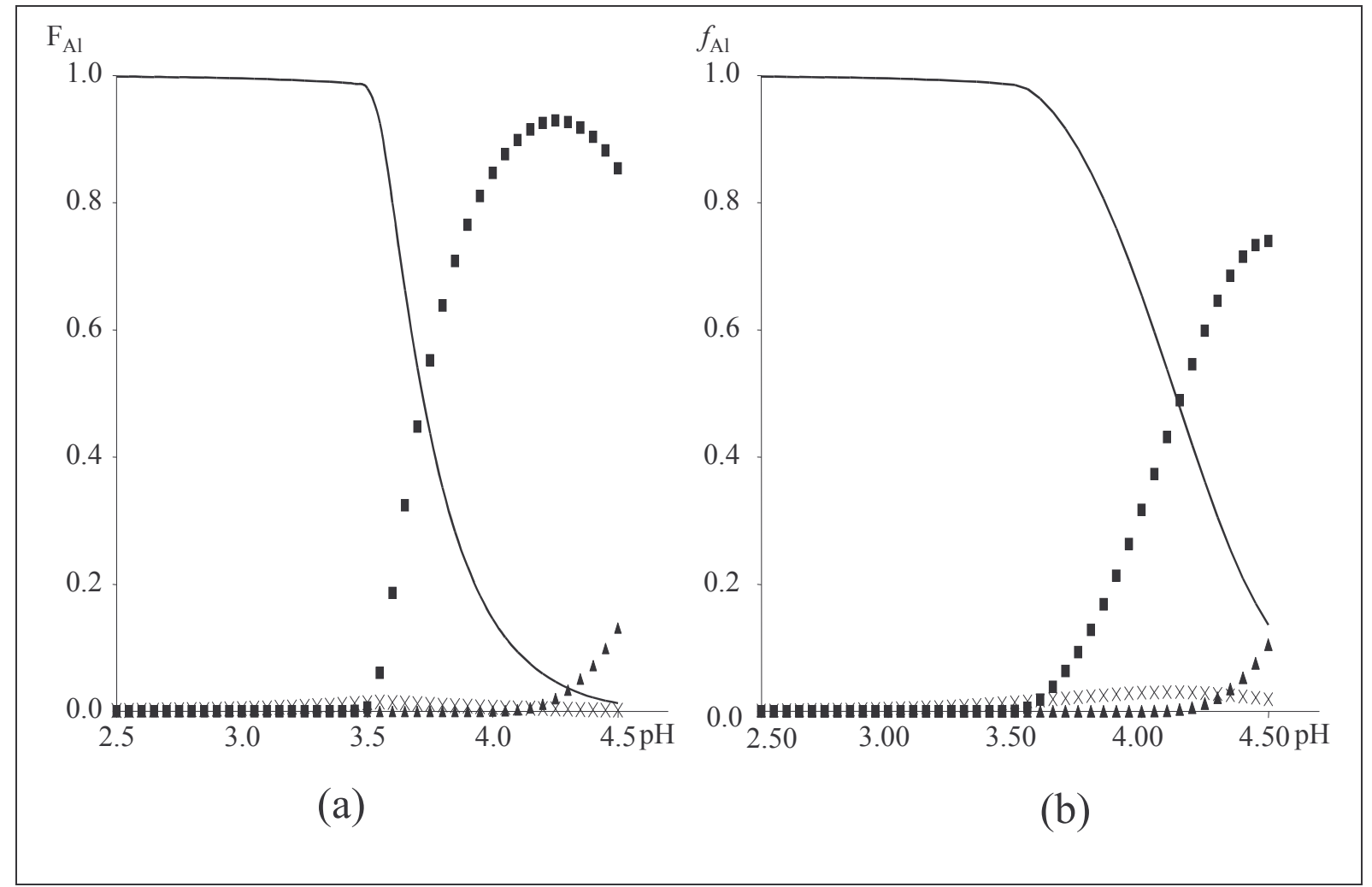

Figura 2.15 (a) Diagrama de fracciones molares componente para especies de Al(III) en el sistema $\mathrm{Al}(\mathrm{III})-\mathrm{Ga}(\mathrm{III})-\mathrm{H}_{2} \mathrm{O}$; (b) Diagrama de fracciones molares de especies de $\mathrm{Al}(\mathrm{III})$ para el sistema $\mathrm{Al}(\mathrm{III})-\mathrm{Ga}(\mathrm{III})-\mathrm{H}_{2} \mathrm{O}$. (Obtenidos a partir del modelo químico propuesto en la Tabla 2.5). (-) $\mathrm{Al}^{3+}$; (X) $\mathrm{Al}(\mathrm{OH})^{2+} ;(\boldsymbol{\Delta}) \mathrm{Al}_{13}(\mathrm{OH})_{32}{ }^{7+} ;(\mathbf{\square}) \mathrm{Al}_{12} \mathrm{Ga}(\mathrm{OH})_{28}{ }^{11+}$. 
Por otro lado, se realizó un estudio cuantitativo de los espectros de ${ }^{27} \mathrm{Al}-\mathrm{NM}$, para lo cual se determinaron las fracciones de área de cada señal mediante integración simple. Las fracciones obtenidas se relacionaron con las fracciones molares de componente y de especie determinadas con base en un modelo químico que considera a las especies determinadas en la Tabla 2.5. Para este propósito se consideraron dos modelos (al igual que para el sistema de $\mathrm{Al}(\mathrm{III})-\mathrm{H}_{2} \mathrm{O}$, ver Capítulo 1) el primero de ellos consiste en relacionar a la fracción de área de la señal de 0ppm de los espectros de RMN con los núcleos de aluminio que poseen una geometría octaédrica, para ello se consideraron las especies monoméricas $\mathrm{Al}\left(\mathrm{H}_{2} \mathrm{O}\right)^{3+}$ y $\mathrm{Al}(\mathrm{OH})^{2+}$, así como una parte ponderada de la fracción molar de la especie $\mathrm{Al}_{13}(\mathrm{OH})_{32}{ }^{7+}$ ya que como se había discutido anteriormente se considera que doce de los núcleos de aluminio poseen esta geometría, además se consideró la fracción molar correspondiente a la especie $\mathrm{Al}_{12} \mathrm{Ga}(\mathrm{OH})_{28}{ }^{11+}$. En la estructura de Keggin los doce aluminios presentes tendrían esta misma geometría octaédrica; y las fracciones de área de la señal de 63 ppm se asociaron con los núcleos de aluminio con geometría tetraédrica que en este caso se encuentran presentes en la especie $\mathrm{Al}_{13}(\mathrm{OH})_{32}{ }^{7+}$ solamente.

El segundo modelo consiste en considerar que la señal de 0ppm se debe a las especies monoméricas presentes en el sistema $\left(\mathrm{Al}\left(\mathrm{H}_{2} \mathrm{O}\right)^{3+}\right.$ y $\left.\mathrm{Al}(\mathrm{OH})^{2+}\right)$ y que la señal en 63 ppm esta relacionada con las especies polinucleares. Se tomó en consideración que esta señal se debe tanto al núcleo tetraédrico como a los doce octaédricos dentro de la especie $\mathrm{Al}_{13}(\mathrm{OH})_{32}{ }^{7+}$, así como a los doce núcleos octahédricos presentes en la especie $\mathrm{Al}_{12} \mathrm{Ga}(\mathrm{OH})_{28}^{11+}$. 
En la Figura 2.16 se muestra la comparación realizada entre las fracciones de área obtenidas de las señales de RMN y de las fracciones molares de componente (F), las cuales fueron calculadas directamente con el programa MEDUSA ${ }^{22}$. Puede apreciarse que cuando se considera el primer modelo propuesto para la comparación (Figura 2.16 a) no existe un buen ajuste entre las fracciones de área experimentales y las determinadas a partir del modelo químico; por el contrario al hacer la comparación con el segundo modelo (Figura 2.16 b) el ajuste es mejor, aunque fue necesario que mediante iteración se variaran las constantes de formación de las especies hasta que las fracciones calculadas ajustaran con aquellas obtenidas experimentalmente (modelo que se muestra en la Tabla 2.7).

Tabla 2.7. Constantes de Formación para el sistema $\mathrm{Al}(\mathrm{III})-\mathrm{Ga}(\mathrm{III})-\mathrm{H}_{2} \mathrm{O}$ determinadas por ajuste gráfico de las fracciones de área de los espectros de $\mathrm{RMN}$ de ${ }^{27} \mathrm{Al}$ con las fracciones molares de componente

\begin{tabular}{cc}
\hline Especie & $-\log * \beta$ \\
\hline $\mathrm{Al}(\mathrm{OH})^{2+}$ & 8.80 \\
$\mathrm{Al}_{13}(\mathrm{OH})_{32}{ }^{7+}$ & 118.00 \\
$\mathrm{Ga}(\mathrm{OH})_{2}^{+}$ & 7.07 \\
$\mathrm{Ga}(\mathrm{OH})_{3}$ & 10.71 \\
$\mathrm{Al}_{12} \mathrm{Ga}(\mathrm{OH})_{28}{ }^{11^{+}}$ & 95.90 \\
\hline
\end{tabular}




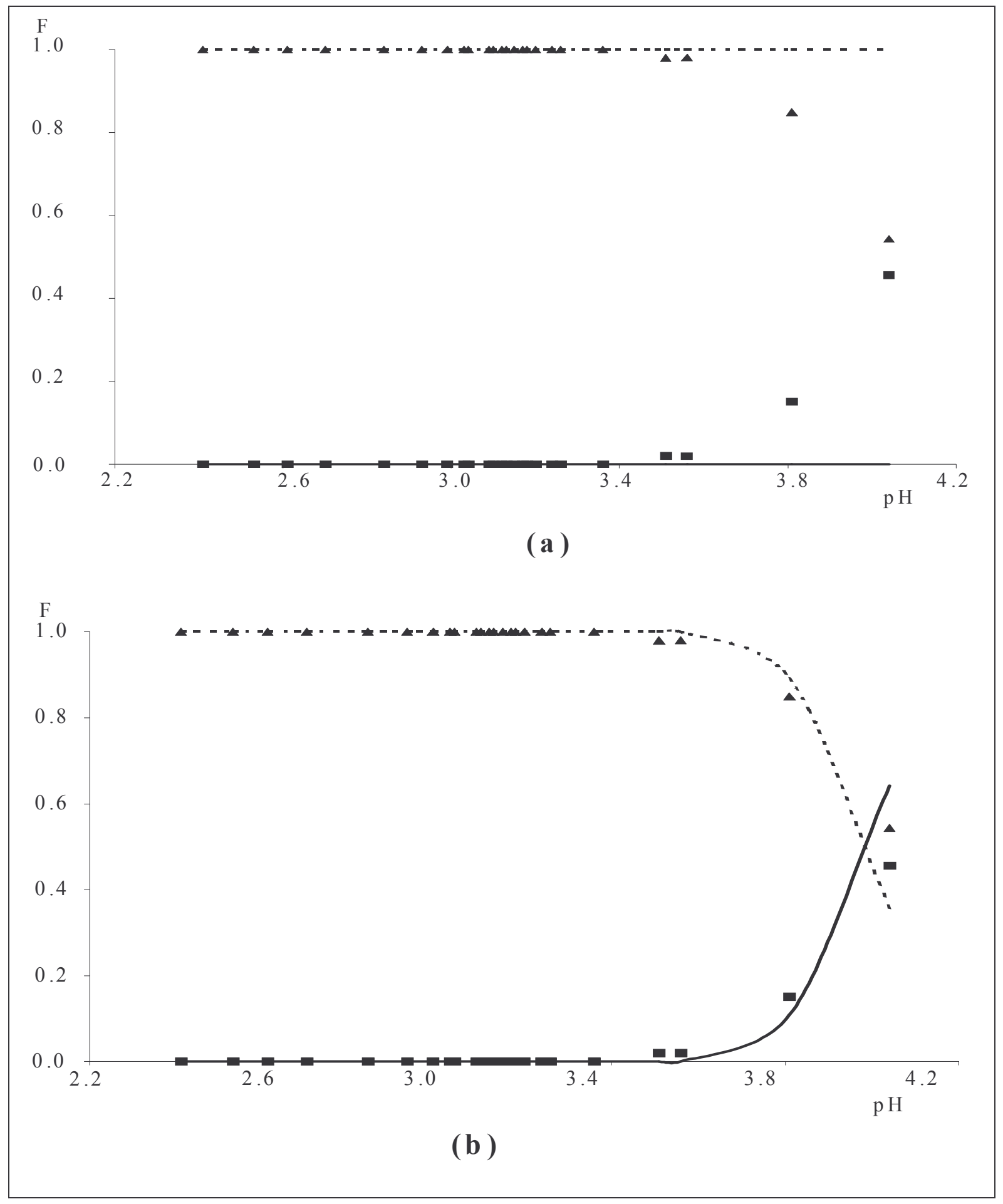

Figura 2.16. Comparación entre la fracciones de área obtenidas experimentalmente de los espectros de RMN de ${ }^{27} \mathrm{Al}$ y las fracciones molares de componente determinadas a partir

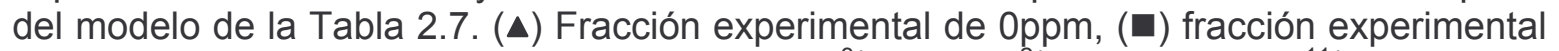
de 63ppm (a) (- -) Fracción estimada de $\mathrm{Al}^{3+}, \mathrm{Al}(\mathrm{OH})^{2+}, \mathrm{Al}_{12} \mathrm{Ga}(\mathrm{OH})_{28}{ }^{11+}$ y fracción proporcional de núcleos octaédricos en $\mathrm{Al}_{13}(\mathrm{OH})_{32}{ }^{7+}$, (-) Fracción estimada para el núcleo tetraédrico en la especie $\mathrm{Al}_{13}(\mathrm{OH})_{32}{ }^{7+}$; (b) (- -) Fracción estimada de $\mathrm{Al}^{3+}, \mathrm{Al}(\mathrm{OH})^{2+},(-)$ Fracción estimada para $\mathrm{Al}_{13}(\mathrm{OH})_{32}{ }^{7+} \mathrm{Al}_{12} \mathrm{Ga}(\mathrm{OH})_{28}{ }^{11+}$ 
Asimismo, se realizó la comparación con las fracciones molares de especie $(f)$ determinadas con base en las ecuaciones 2.6 y 2.7 con ayuda del programa computacional MEDUSA y una hoja de cálculo para la aplicación Excel de MS-Office97 (Microsoft). La Figura 2.17a muestra, al igual que en el caso anterior, que al considerar que la señal de 0 ppm se debe a todos los núcleos octaédricos de aluminio, y que la señal de 63ppm está relacionada únicamente con los núcleos tetraédricos (modelo 1) no existe un ajuste entre las fracciones. Por otro lado, la Figura $2.17 \mathrm{~b}$ permite apreciar que con el segundo modelo, esto establece que la señal de 0 ppm se relaciona con las especies monoméricas, mientras que la señal de 63 ppm es debida a las especies polinucleares. El ajuste es mucho mejor y en este caso las constantes también fueron variadas iterativamente hasta lograr la mejor concordancia entre las fracciones correspondientes. El modelo químico que permite esto se muestra en la Tabla 2.8

$$
\begin{aligned}
& {\left[\mathrm{Al}_{\mathrm{i}}(\mathrm{OH})_{\mathrm{k}}^{3 \mathrm{i}-\mathrm{k}}\right]=\frac{\mathrm{F}_{\mathrm{Al}_{\mathrm{i}}(\mathrm{OH})_{\mathrm{k}}^{3 \mathrm{i}-\mathrm{k}}}\left[\mathrm{Al}^{3+}\right]_{\text {inicial }}}{\mathrm{i}}} \\
& {\left[\mathrm{Al}_{\mathrm{i}} \mathrm{Ga}_{\mathrm{j}}(\mathrm{OH})_{\mathrm{k}}{ }^{3(\mathrm{i}+\mathrm{j})-\mathrm{k}}\right]=\frac{\mathrm{F}_{\mathrm{Al}_{\mathrm{i}} \mathrm{Ga}_{\mathrm{j}}(\mathrm{OH})_{\mathrm{k}}{ }^{3(\mathrm{i}+\mathrm{j})-\mathrm{k}}\left[\mathrm{Al}^{3+}\right]_{\text {inicial }}}^{\mathrm{i}}}{\mathrm{i}}} \\
& \boldsymbol{f}_{\mathrm{Al}_{\mathrm{i}} \mathrm{Ga}_{\mathrm{j}}(\mathrm{OH})_{\mathrm{k}}{ }^{3(\mathrm{i}+\mathrm{j})-\mathrm{k}}}=\frac{\mathrm{F}_{\mathrm{Al}_{\mathrm{i}} \mathrm{Ga}_{\mathrm{j}}(\mathrm{OH})_{\mathrm{k}}{ }^{3(\mathrm{i}+\mathrm{j})-\mathrm{k}}}\left[\mathrm{Al}^{3+}\right]_{\text {inicial }} / \mathrm{i}}{\left(\sum_{\mathrm{i}}\left(\mathrm{F}_{\mathrm{Al}_{\mathrm{i}}(\mathrm{OH})_{\mathrm{k}}{ }^{3 \mathrm{i}-\mathrm{k}}}\left[\mathrm{Al}^{3+}\right]_{\text {inicial }} / \mathrm{i}\right)\right)+\left(\mathrm{F}_{\mathrm{Al}_{\mathrm{i}} \mathrm{Ga}_{\mathrm{j}}\left(\mathrm{OH}_{\mathrm{k}}{ }^{3(\mathrm{i}+\mathrm{j})-\mathrm{k}}\right.}\left[\mathrm{Al}^{3+}\right]_{\text {inicial }} / \mathrm{i}\right)}
\end{aligned}
$$




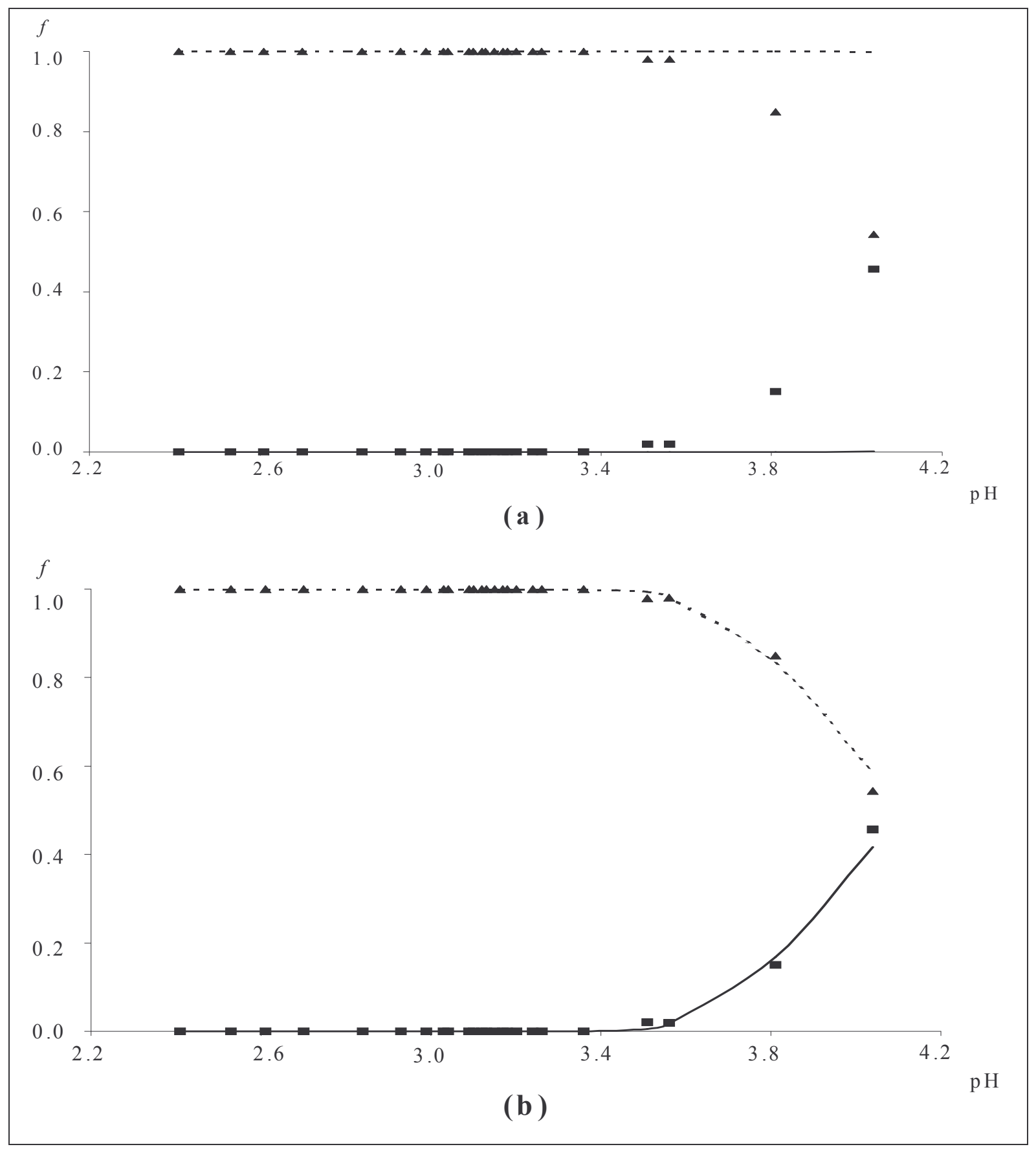

Figura 2.17. Comparación entre la fracciones de área obtenidas experimentalmente de los espectros de RMN de ${ }^{27} \mathrm{Al}$ y las fracciones molares de especie determinadas a partir del

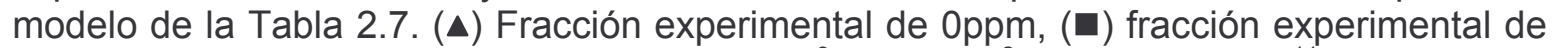
63ppm (a) (- -) Fracción estimada de $\mathrm{Al}^{3+}, \mathrm{Al}(\mathrm{OH})^{2+}, \mathrm{Al}_{12} \mathrm{Ga}(\mathrm{OH})_{28}{ }^{1+}$ y fracción proporcional de núcleos octaédricos en $\mathrm{Al}_{13}(\mathrm{OH})_{32}{ }^{7+}$, (-) Fracción estimada para el núcleo tetraédrico en la especie $\mathrm{Al}_{13}(\mathrm{OH})_{32}{ }^{7+}$; (b) (- -) Fracción estimada de $\mathrm{Al}^{3+}, \mathrm{Al}(\mathrm{OH})^{2+},(-)$ Fracción estimada para $\mathrm{Al}_{13}(\mathrm{OH})_{32}{ }^{7+} \mathrm{Al}_{12} \mathrm{Ga}(\mathrm{OH})_{28}{ }^{1+}$ 
Tabla 2.8. Constantes de Formación para el sistema $\mathrm{Al}(\mathrm{III})-\mathrm{Ga}(\mathrm{III})-\mathrm{H}_{2} \mathrm{O}$ determinadas por ajuste gráfico de las fracciones de área de los espectros de $\mathrm{RMN}$ de ${ }^{27} \mathrm{Al}$ con las fracciones molares de especie

\begin{tabular}{cc}
\hline Especie & $-\log * \beta$ \\
\hline $\mathrm{Al}(\mathrm{OH})^{2+}$ & 5.50 \\
$\mathrm{Al}_{13}(\mathrm{OH})_{32}{ }^{7+}$ & 110.00 \\
$\mathrm{Ga}(\mathrm{OH})_{2}{ }^{+}$ & 7.07 \\
$\mathrm{Ga}(\mathrm{OH})_{3}$ & 10.04 \\
$\mathrm{Al}_{12} \mathrm{Ga}(\mathrm{OH})_{28}{ }^{11+}$ & 88.30 \\
\hline
\end{tabular}

De la comparación realizada tanto con las fracciones molares de componente como con las fracciones molares de especie, se puede ver que en ambos casos al relacionar la señal de 0ppm con núcleos de aluminio octaédricos y la de 63 ppm con núcleos con geometría tetraédrica no se presenta una sobreposición con las fracciones molares calculadas. Lo anterior permite establecer que el mejor modelo de ajuste es el que establece que la primer señal esta relacionada con las especies mononucleares y que la segunda señal observada $(63 \mathrm{ppm})$ se relaciona con los núcleos de aluminio de las especies polinucleares $\left(\mathrm{Al}_{13}(\mathrm{OH})_{32}{ }^{7} \mathrm{y} \mathrm{Al}_{12} \mathrm{Ga}(\mathrm{OH})_{28}{ }^{1+}\right)$. De alguna manera la señal se debe a los núcleos con geometría octaédrica del aluminio presentes en estas especies y no es una señal debida a núcleos tetraédricos como establece Akitt y colaboradores ${ }^{42}$. Esto explica entonces la naturaleza química de la señal en el sistema de $\mathrm{Al}(\mathrm{III})-\mathrm{H}_{2} \mathrm{O}$ analizada en el capítulo anterior. 
Por otro lado, para la comparación con las fracciones molares de componente si bien se llega a un adecuado ajuste de las fracciones calculadas con las experimentales fue necesario modificar notablemente el valor de las constantes de formación de las especies en relación con aquellos valores obtenidos para las especies determinadas con el programa SUPERQUAD a partir de información potenciométrica (Tabla 2.5). Mientras que para las fracciones molares de especie los valores de las constantes para lograr el ajuste son muy similares a las determinadas en el estudio potenciométrico. Esto permite establecer que las señales obtenidas en el estudio de ${ }^{27} \mathrm{Al}-\mathrm{RMN}$ para el sistema $\mathrm{Al}(\mathrm{III})-\mathrm{Ga}(\mathrm{III})-\mathrm{H}_{2} \mathrm{O}$ son el producto del promedio de las señales de las molares de especie de los núcleos de aluminio, como ya había sido probado para los sistemas de $\mathrm{Al}(\mathrm{III})-\mathrm{H}_{2} \mathrm{O}$ y de $\mathrm{B}(\mathrm{III})-\mathrm{H}_{2} \mathrm{O}$ en el estudio de los sistemas binarios (Capítulo I).

\subsubsection{CONCLUSIONES}

Los estudios realizados en este trabajo nos permiten validar el modelo propuesto en la Tabla 2.5 para el sistema $\mathrm{Al}(\mathrm{III})-\mathrm{Ga}(\mathrm{III})-\mathrm{H}_{2} \mathrm{O}$, ya que con base en el es posible explicar el comportamiento observado en valoraciones conductimétricas y en estudios de RMN para

${ }^{71} \mathrm{Ga} \mathrm{y}{ }^{27} \mathrm{Al}$. Además fue posible confirmar las constantes de las especies $\mathrm{Al}(\mathrm{OH}){ }^{2+} \mathrm{y}$ $\mathrm{Al}_{13}(\mathrm{OH})_{32}{ }^{7+}$ del sistema $\mathrm{Al}(\mathrm{III})-\mathrm{H}_{2} \mathrm{O}$ y Ga(OH $)_{2}{ }^{+}$y $\mathrm{Ga}(\mathrm{OH})_{3}$ del sistema $\mathrm{Ga}(\mathrm{III})-\mathrm{H}_{2} \mathrm{O}$, y se puso en evidencia la formación del complejo ternario de alto peso molecular $\mathrm{Al}_{12} \mathrm{Ga}(\mathrm{OH})_{28}{ }^{11+}$ (cuyo valor de constante formación es $-\log * \beta=88.66 \pm 0.15$ ). Esta especie podría ser de gran importancia ya que puede considerarse un precursor químico que permita la obtención de sistemas catalíticos mesoporosos. 
Por otro lado, se determinó con base en estudios potenciométricos y de RMN que las especies $\mathrm{Al}(\mathrm{OH})_{3}$ y $\mathrm{Al}_{3}(\mathrm{OH})_{4}{ }^{5+}$ no se forman en este sistema debido a la formación del complejo ternario. Se pudo establecer que la señal de 63ppm observada en los espectros de RMN esta relacionada con núcleos de aluminio octaédricos presentes en las especies polinucleares siendo probable que el amplio desplazamiento de esta señal hacia campos bajos en relación a la zona en donde típicamente estarían las señales para núcleos con esta geometría se deba a la distorsión en el ambiente químico generado por los núcleos tetraédricos presentes. 


\section{CONCLUSIONES GENERALES}

A través del desarrollo de este trabajo se estableció una metodología para la determinación de modelos químicos para sistemas tanto binarios como ternarios, en particular se estudiaron los procesos de hidrólisis de elementos del grupo IIIA (boro, aluminio, galio, indio), así como de los sistemas $\mathrm{Al}(\mathrm{III})-\mathrm{B}(\mathrm{III})-\mathrm{H}_{2} \mathrm{O}, \mathrm{Al}(\mathrm{III})-\mathrm{Ga}(\mathrm{III})-\mathrm{H}_{2} \mathrm{O}$ y $\mathrm{Al}(\mathrm{III})-\mathrm{In}(\mathrm{III})-\mathrm{H}_{2} \mathrm{O}$ obteniendo para todos ellos las posibles especies químicas, así como sus constantes de formación respectivas. La importancia de los modelos obtenidos en comparación con otros ya reportados en la literatura, se debe a que en este trabajo estos no solamente fueron determinados mediante el procesamiento con el programa computacional SUPERQUAD de información potenciométrica sino que también fueron validados mediante otros estudios y técnicas analíticas como análisis de la función de formación $(\mathrm{Z})$, y estudios por medio de conductimetría y de resonancia magnética nuclear. Con los estudios de RMN se aportó un soporte desde el punto de vista estructural a los modelos químicos obtenidos. Con ello se corroboró que los modelos químicos propuestos permiten explicar el comportamiento experimental de los sistemas por diferentes técnicas analíticas lo cual da soporte y validez a los datos obtenidos en el presente trabajo.

Adicionalmente, en este trabajo se presenta un nuevo algoritmo que da evidencia cuantitativa suficiente para establecer que las señales de RMN están relacionadas (al menos para $\mathrm{B}(\mathrm{III})$ y $\mathrm{Al}(\mathrm{III})$ ) con las fracciones que dependen de la concentración de las especies 
presentes en el sistema, estableciendo que las áreas bajo los espectros de RMN son directamente proporcionales a la concentración de las especies.

En este trabajo se emplea el algoritmo propuesto con la finalidad de dar validez a algunos de los modelos químicos propuestos dando no solo información sobre la geometría de las especies presentes, sino también un significado estructural a los modelos, además de un soporte cuantitativo a los modelos químicos al poder interpretar cuantitativamente las señales de RMN.

Mediante este trabajo se establece que las señales de RMN tienen relación directa con las fracciones molares de las especies y por tanto con las concentraciones de las especies que se forman. Lo anterior permite sugerir que se podría plantear una generalización de la ley de Beer y la ley de las aditividades (como sucede en otras espectroscopias), lo cual puede no solo brindar una metodología para la determinación de especies y sus constantes de formación asociadas, sino también fincar las bases para el desarrollo de métodos cuantitativos de análisis empleando esta técnica. 


\section{PROYECCIÓN DEL TRABAJO}

La caracterización química de los sistemas analizados en este trabajo puede adquirir especial importancia ya que este trabajo esta vinculado con estudios básicos que buscan el poder establecer las condiciones químicas adecuadas para tener a determinadas especies químicas en solución, en especial las especies polinucleares tanto de sistemas binarios como de ternarios, para que estas puedan ser empleadas como precursores en el proceso de impregnación para el pilareo de arcillas que pudieran ser utilizadas en procesos de catálisis de hidrocarburos. De esta manera el contar con un modelo químico confiable para el sistema permite establecer las condiciones de hidrólisis adecuadas para la formación y predominio de una especie química de interés para la impregnación de las arcillas o para cualquier otra aplicación tecnológica y evitar con ello el tener que determinar empíricamente las condiciones óptimas de trabajo, lo cual implica tiempo y en muchas ocasiones gastos. 


\section{BIBLIOGRAFÍA}

[1] Ch. F. Baes Jr. and R. E. Mesmer. The Hydrolysis of Cations, Wiley, USA (1976).

[2] Hartley F. R., Burgess C. and Alcock R. M. Solution Equilibria. John Wiley and Sons, Great Britain (1980)

[3] Cheng Soofin. Cat. Today. 49, 308 (1999)

[4] Sapag K., Mendioroz S. Colloids and Sur. A. Phys. And Eng. Asp., 141 (2001)

[5] Occelli M. L., Auroux A., Ray G. J. Micro. Meso. Mat., 39, 43 (2000)

[6] Occelli M. L., Bertrand J. A., Gould S. A. C., Domínguez J. M. Micro. Meso. Mat., 34, $195(2000)$

[7] Bottero J. Y., Cases J. M., Flessinger F. and Potrier J. E., J. Phys. Chem., 84, 2933 (1980)

[8] Öhman O. and Forsling W., Acta Chem. Scan., A35, 795 (1981)

[9] D.E.W. Vaughan. Catalysis Today. 2, 187 (1988).

[10] M.L. Occelli. Keynotes in Energy Related Catalysis. ed. S. Kaliaguine, Elsevier. $1988,101$.

[11] R.M. Barrer. Phi. Trans. R. Soc. London. A 311, 333 (1984).

[12] M.L. Occelli. Ind. Eng. Chem. Prod. Res. Dev. 22, 553 (1983).

[13] M.L. Occelli and R.M. Tindwa. Clays Clay Miner. 31, 22 (1983).

[14] C. E. Ramos-Galván, G. Sandoval-Robles, A. Castillo-Mares and J. M. Domínguez, Applied Catal. 43, 69 (1996).

[15] J. Flanagan, W. P. Griffith, R. D. Powell and A. P. West, J. Chem. Soc. Dalton Trans., 1651 (1989)

[16] B. Owen and E. King, J. Am. Chem. Soc., 56, 937 (1943)

[17] N. Ingri. Acta Chem. Scand. 1962, 16, 439.

[18] V. Gold and B. Lowe, J. Chem. Soc., A (1923)

[19] K. Khoo, J. Chem. Soc., A 1177, 2932 (1971)

[20] D. Feakins and K. Khoo, J. Chem. Soc., A 361 (1970)

[21] J. Spessard, J. Inorg. Nucl. Chem., 32, 2607 (1970)

[22] G. Crisponi, V. Nurchi, M. Ganadu and G. Lubinu, Polyhedron, 9, 789 (1990) 
[23] H. Rogers and C. Van der Berg, Talanta, 35, 271 (1988)

[24] A. Sabatini, A. Vacca and P. Gans. Talanta. 1974, 21, 53

[25] Gans P., Sabatini A. and Vacca A., J. Chem. Soc. Dalton Trans., 1195 (1985)

[26] D. Hollins. Biological Magnetic Resonance. vol 2, Plenum Press, USA (1980)

[27] M .Guzmán and J. J. Hicks. Ciencia. 42, 207 (1991)

[28] K. Ishihara, A. Nagasawa, K. Umemoto, H. Ito and K. Saito. Inorganic Chemistry, 33, 3811 (1994)

[29] I. Puigdomenech, MEDUSA (Making Equilibrium Diagrams Using Sophistica-ted Algorithms) Royal Institute of Technology. Stockholm, Sweden

[30] Botello J. C., Morales-Domínguez E., Domínguez J. M., Gutiérrez A., RojasHernández A.,Ramírez M. T. Spectrochim. Acta. Part A, In press

[31] Schoefield K. and A. W. Taylor, J Chem Soc., 4445 (1954).

[32] Ito and N Yui, Chem. Abs., 48, 5613c (1954)

[33] Aveston J., J. Chem. Soc., 4438 (1965).

[34] Frink R. and Peech M., Inor. Chem., 2, 3, 473 (1963)

[35] R. C. Tuner, Can. J. Chem, 53, 19, 2811 (1975)

[36] Charlet, Deloume J. P., Duc G., Thomas-David G., Bull. Soc. Chim. France, 7-8, I222-I226 (1984).

[37] Tóth Y., Zékany L. and Brücher E., Polyhedron, 7, 3, 871 (1984)

[38] Jorge Ares, Anal. Chim. Acta, 187, 181 (1986)

[39] Brown P., Sylva R. N. and Batley G. E., J. Chem. Soc. Dalton Trans., 1968 (1985)

[40] Letterman R. D. and Asolekar S. R:, Wat. Res., 24, 8, 941 (1990)

[41] Marklund E. y Öhman L. Acta Chem. Scandinavica, 44, 228 (1990)

[42] J.W. Akitt and N.N. Greenwood, B. L. Khandelwal and D. Lester. J. Chem. Soc. Dalton Trans., 604 (1972)

[43] J. W. Akkit. and A. Farthing ., J. Chem. Soc. Dalton Trans., 1617 (1981).

[44] Akkit J. W., Elders J. M. and Fountaine L. R., J. Chem. Soc. Dalton Trans., 1047 (1986).

[45] Akkit J. W.and Elders J. M., J. Chem. Soc. Dalton Trans., 1347 (1988).

[46] Akkit J. W., Elders J. M., Fountaine L. R. and Kundo A. M., J. Chem. Soc. Dalton Trans., 1889 (1989). 
[47] Akkit J. W., Elders J. M., Fountaine L. R. and Kundo A. M., J. Chem. Soc. Dalton Trans., 1897 (1989)

[48] J. M. Domínguez, J. C. Botello-Pozos, A. López-Ortega, M. T. Ramírez, G. Sandoval-Flores and A. Rojas-Hernández. Catalysis Today. , 43, 69 (1998)

[49] M. L. Pacheco-Hernández, Tesis de Maestría, UAM-I, México (1999)

[50] Castellan G. W., Fisicoquímica, 2de, Addison-Wesley Iberoamericana, U.S.A. (1983)

[51] Wilson A. S. and Taube H., J. Amer. Chem. Soc., 74, 3509 (1952).

[52] Nazarenko V. A. and Antonovich V. P., Inor. Chem., 13, 825 (1968).

[53] Yamada S., Iwanaya A., Funahashi s. and Tanaka M., Inor. Chem., 23, 3528 (1984)

[54] Campisi A. and Tregloan P., Inor. Cem. Acta, 100, 251 (1985)

[55] P. L. Brown and R. N. Sylva. J. Chem. Soc. Dalton Trans. 1985 (1967).

[56] B. Borgias, A. Hugi and K. Raymond. Inorg. Chem., 1989, 28, 3538.

[57] Secretaria de Salud, Farmacopea de los Estados Unidos Mexicanos, 5ed, México (1988)

[58] Hartley F. R., Burgess C. and Alcock R. M. Solution Equilibria. John Wiley and Sons, Great Britain (1980)

[59] F. Rossotti and H. Rossotti, Acta Chem. Scand., 10, 779 (1956)

[60] G. Biedermann, N. Li., J. Yu , Acta Chem. Scand., 15, 555 (1961)

[61] A. Aziz and S. Lyle, J. Inorg. Nucl. Chem., 31, 2431 (1969)

[62] G. Biedermann and D. Ferri, Acta Chem. Scand., A36, 661 (1982)

[63] Brown P., J. Ellis, R. Sylva, J. Chem. Soc. Dalton Trans, 1911 (192)

[64] S.M. Bradley, R.A: Kydd, R. Yamdagni. J. Chem. SOc., Dalton Trans. 2653 (1990)

[65] B. Thomas, H. Görz and S. Schönherr. Z. Chem. 27, 183 (1987).

[66] Ramette Richard W. Equilibrio y Análisis Químico. Fondo Educativo Interamericano, España (1983)

[67] Ringbom A. Formación de Complejos en Química Analítica. Alhambra, España

[68] Moya Hernández. R. Tesis de maestría. FES-Cuatitlán, UNAM. Mexico (1997)

[69] .Sillén G. Treatrise on Analytical Chemistry. I. M. Kolthoff and P. J. Elving Eds. Vol. 1. Part B. Wiley. New York (1959). 
[70] Contreras T. R., Importancia de la RMN en la Química Inorgánica, Coloquio: Técnicas Analíticas Importantes en Química Inorgánica, AMQI, México (1993)

[71] Mason J., Multinuclear NMR, Plenum Press, USA (1987)

[72] Nathan J. y Díaz E. Introducción a la Resonancia Magnética Nuclear, Limusa, México (1980). 


\section{APÉNDICE 1 \\ EQUILIBRIO DE HIDRÓLISIS Y MÉTODOS GRÁFICOS PARA SU REPRESENTACIÓN}

\section{A.1.1. EQUILIBRIO DE HIDRÓLISIS ${ }^{1,66}$}

Un tipo particular de equilibrio químico de interés en diversos ámbitos de la ciencia y la tecnología es el proceso de hidrólisis, este se refiere a las reacciones químicas en las cuales una substancia es "descompuesta" por el agua. La hidrólisis de especies inorgánicas se refiere a las reacciones que tienen lugar en soluciones de sales, en las cuales son formadas nuevas especies ionicas o precipitados como óxidos o hidróxidos. La hidrólisis de sales puede involucrar tanto al catión como al anión o bien a ambos, aunque generalmente los estudios se enfocan a la hidrólisis de cationes para formar especies solubles con hidróxido u oxi-complejos, así como la formación de precipitados de hidróxido.

Prácticamente todos los cationes son capaces de formar hidroxo-complejos, lo cual no debe sorprendernos si consideramos que el ligando $\mathrm{OH}^{-}$esta siempre presente en el agua, en concentraciones que pueden variar en el intervalo de $10^{-1} \leq\left[\mathrm{OH}^{-}\right] \leq 10^{-14}$ esto como resultado de la disociación del agua, el valor de la concentración de los iones hidróxilo dependerá por tanto del $\mathrm{pH}$ del sistema $\mathrm{Al}$ ion hidróxilo se le clasifica como un ligante fuerte, que puede formar complejos muy estables con cationes metálicos de transición menos polarizables ("cationes fuertes") a través de la formación de enlaces predominantemente ionicos. Sin embargo, también se pueden formar hidroxocomplejos 
con los llamados “cationes suaves" de los metales de post-transición, formando enlaces con un apreciable carácter covalente.

La ecuación que describe la reacción de formación de productos solubles en el proceso de hidrólisis, así como la expresión de la constante de equilibrio respectiva (la cual generalmente se etiqueta con la letra griega $\beta$ ) se muestran en la ecuación A.1.1, debido a la diversidad de complejos que se pueden formar, el comportamiento del metal en una solución puede ser difícil de interpretar y este será función tanto del $\mathrm{pH}$ como de la concentración, así como de la identidad y estabilidad de los productos formados en el proceso de hidrólisis.

$$
\begin{gathered}
i M^{z+}+j H_{2} \mathrm{O} \leftrightarrow M_{i}(\mathrm{OH})_{j}^{(i z-j)}+j H^{+} \\
* \beta_{i, j}=\frac{\left[M_{x}(O H)_{j}^{(i z-j)}\right]\left[H^{+}\right]^{j}}{\left[M^{z+}\right]^{i}}
\end{gathered}
$$

La determinación de las especies que se forman durante la hidrólisis, así como sus constantes de equilibrio generalmente esta acompañado de algunas dificultades como son:

(3) Los hidroxocomplejos formados pueden ser especies tanto mononucleares como polinucleares, es decir especies que contienen más de un ion metálico. La diversidad de especies posibles y la aparición de estas en mayor o menos proporción de manera simultanea dificulta su caracterización y determinación de su estabilidad. 
(4) El intervalo de $\mathrm{pH}$ en el cual se puede estudiar la formación de los hidroxocomplejos puede, en ocasiones, ser limitado por la precipitación del hidróxido o el oxido del ion metálico.

Sin embargo, pese a las dificultades antes señaladas se han desarrollado desde principios del siglo XX numerosos estudios para la identificación y caracterización de diversas especies formadas en la hidrólisis de la mayoría de los iones metálicos, actualmente las experimentaciones que permitan conocer la naturaleza de la especies químicas, así como sus constantes de formación se siguen desarrollando por diversas técnicas instrumentales y/o con la ayuda de programas computacionales.

\section{A.1.1.1. Especies mononucleares ${ }^{1,2}$}

Estas especies se forman con prácticamente todos los cationes, pero los metales alcalinos pueden formar uno o más productos de hidrólisis mononucleares, son especies cuya formación se puede describir mediante la ecuación A.1.2.

$$
\begin{gathered}
M^{z+}+j H_{2} O \leftrightarrow M(O H)_{y}^{(z-j)}+j H^{+} \\
* \beta_{j}=\frac{\left[M(O H)_{j}^{(z-j)}\right]\left[H^{+}\right]^{j}}{\left[M^{z+}\right]}
\end{gathered}
$$




\section{A.1.1.2. Especies polinucleares ${ }^{1,2}$}

Los puentes que el hidróxido puede formar parecen ser los responsables de la formación de casi todas las especies polinucleares de los cationes di-, tri- y tetra-valentes siendo el ion hidróxido compartido con dos o tres cationes adyacentes. La formación de estas especies se puede describir de manera general mediante la ecuación A.1, en la cual se asume que por cada $\mathrm{OH}^{-}$que se tenga este será compartido con $x$ átomos del catión. Las configuraciones de las especies polinucleares son aquellas que muestran simetría. Muchas de las estructuras pueden ser ensambladas por dos, tres, cuatro, cinco y seis átomos manteniéndose unidos por medio del ion hidróxido

\section{A.1.2. DIAGRAMAS DE DISTRIBUCIÓN}

\section{A.1.2.1. Fracciones Molares de Componente ${ }^{1,2,67}$}

Existen diversas representaciones gráficas para poder mostrar el comportamiento o el perfil de las especies en función del pH del sistema, estas puede realizarse con base a las concentraciones de las especies, de las cantidades de estas o bien en función de las fracciones molares. Probablemente una de las representaciones gráficas más empleadas son los diagramas de distribución de especies en los cuales se muestra el comportamiento de las fracciones molares como una función del pH (en el caso de procesos de hidrólisis, o bien cuando el sistema depende del pH).

La representación gráfica más empleada propone el cálculo de las fracciones molares de componente (también conocida como fracciones de cantidad), ellas determinan 
la fracción de cantidad de un componente que cada especie tiene con relación al total de dicho componente en la solución o sistema, estas se describen de acuerdo a la ecuación A.1.3.

$$
F_{i j}=F_{M_{i}(O H)_{j}^{3 i-j}}=\frac{i\left[M_{i}(O H)_{j}^{3 i-j}\right]}{[M]_{\text {total }}}=\frac{i\left[M_{i}(O H)_{j}^{3 i-j}\right]}{\sum_{i}\left(\sum_{j}\left\{i\left[M_{i}(O H)_{j}^{3 i-j}\right]\right\}\right)}
$$

Para comprender mejor lo anterior consideremos un primer ejemplo en el que se tiene el proceso de hidrólisis del catión $\mathbf{M}$ descrito por la ecuación A.1.1, formándose dos complejos mononucleares, uno de estequiometría 1:1 y otro más de estequiometría 1:2, los equilibrios y sus expresiones de constantes de formación así como los valores supuestos de estas ultimas son las siguientes:

$$
\begin{aligned}
& \mathrm{M}^{\mathrm{z+}}+\mathrm{H}_{2} \mathrm{O} \leftrightarrow \mathrm{M}(\mathrm{OH})^{(\mathrm{z}-1)}+\mathrm{H}^{+} * \beta_{1}=\frac{\left[\mathrm{M}(\mathrm{OH})^{(\mathrm{z}-1)}\right]\left[\mathrm{H}^{+}\right]}{\left[\mathrm{M}^{\mathrm{z}}\right]}=10^{-4.2} \\
& \mathrm{M}^{\mathrm{z+}}+2 \mathrm{H}_{2} \mathrm{O} \leftrightarrow \mathrm{M}(\mathrm{OH})_{2}^{(\mathrm{z}-2)}+2 \mathrm{H}^{+} \quad * \beta_{2}=\frac{\left[\mathrm{M}(\mathrm{OH})_{2}^{(\mathrm{z}-2)}\right]\left[\mathrm{H}^{+}\right]^{2}}{\left[\mathrm{M}^{\mathrm{z}}\right]}=10^{-145}
\end{aligned}
$$

las fracciones molares de componente para cada una de las especies están definidas de acuerdo al conjunto de ecuaciones A.1.5. 


$$
\begin{aligned}
& \mathrm{F}_{\mathrm{M}}=\frac{\left[\mathrm{M}^{\mathrm{z}}\right]}{\left[\mathrm{M}^{\mathrm{z+}}\right]+\left[\mathrm{M}(\mathrm{OH})^{\mathrm{z}-1}\right]+\left[\mathrm{M}(\mathrm{OH})_{2}^{\mathrm{z}-2}\right]} \\
& \mathrm{F}_{\mathrm{M}(\mathrm{OH})^{z-1}}=\frac{\left[\mathrm{M}(\mathrm{OH})^{\mathrm{z}-1}\right]}{\left[\mathrm{M}^{\mathrm{z}+}\right]+\left[\mathrm{M}(\mathrm{OH})^{\mathrm{z}-1}\right]+\left[\mathrm{M}(\mathrm{OH})_{2}^{\mathrm{z}-2}\right]} \\
& \mathrm{F}_{\mathrm{M}(\mathrm{OH})_{2}^{z-2}}=\frac{\left[\mathrm{M}(\mathrm{OH})_{2}^{\mathrm{z}-2}\right]}{\left[\mathrm{M}^{\mathrm{z+}}\right]+\left[\mathrm{M}(\mathrm{OH})^{\mathrm{z}-1}\right]+\left[\mathrm{M}(\mathrm{OH})_{2}^{\mathrm{z}-2}\right]}
\end{aligned}
$$

si en las expresiones anteriores sustituimos las concentraciones determinadas a partir de A.1.4 se obtiene:

$$
\begin{aligned}
& \mathrm{F}_{\mathrm{M}^{2+}}=\frac{1}{1+* \beta_{1} /\left[\mathrm{H}^{+}\right]+* \beta_{2} /\left[\mathrm{H}^{+}\right]^{2}} \\
& \mathrm{~F}_{\mathrm{M}(\mathrm{OH})^{2-1}}=\frac{* \beta_{1} /\left[\mathrm{H}^{+}\right]}{1+* \beta_{1} /\left[\mathrm{H}^{+}\right]+* \beta_{2} /\left[\mathrm{H}^{+}\right]^{2}} \\
& \mathrm{~F}_{\mathrm{M}(\mathrm{OH})_{2}^{z-2}}=\frac{* \beta_{2} /\left[\mathrm{H}^{+}\right]^{2}}{1+* \beta_{1} /\left[\mathrm{H}^{+}\right]+* \beta_{2} /\left[\mathrm{H}^{+}\right]^{2}}
\end{aligned}
$$

en estas ecuaciones se puede apreciar que en sistemas en donde solamente se forman especies mononucleares dichas fracciones son independientes de la concentración del ion metálico, el diagrama correspondiente se puede apreciar en la figura A.1.1. 


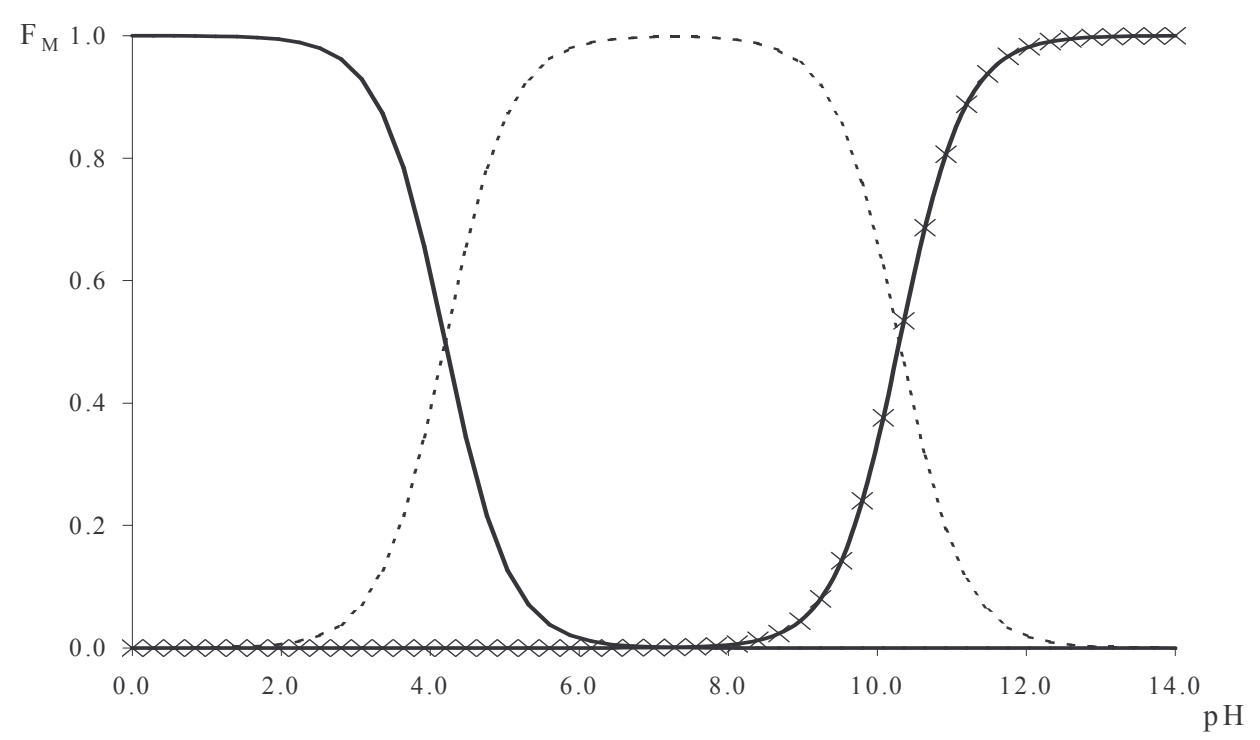

Figura A.1.1. Diagrama de distribución de fracciones de componente para un sistema en el que los productos de hidrólisis son $\mathrm{M}(\mathrm{OH})^{\mathrm{z}-1}$ y $\mathrm{M}(\mathrm{OH})_{2}{ }^{\mathrm{z}-2}$ con $-\log * \beta$ de 4.2 y 14.5 respectivamente ( - ) fracción molar de $\mathrm{M}^{\mathrm{z}+}$, ( - - ) fracción molar de $\mathrm{M}(\mathrm{OH})^{\mathrm{z}-1}$, (-X-) fracción molar de $\mathrm{M}(\mathrm{OH})_{2}^{\mathrm{z}-2}$.

Consideremos ahora un sistema en el que se forma una especie mononuclear de estequiometría $(1,1)$, así como dos especies polinucleares, un dímero $(2,2)$ y un trímero $(3,4)$, cuyos equilibrios de formación están descritos por A.1.7.

$$
\begin{aligned}
& \mathrm{M}^{\mathrm{z+}}+\mathrm{H}_{2} \mathrm{O} \leftrightarrow \mathrm{M}(\mathrm{OH})^{(\mathrm{z}-1)}+\mathrm{H}^{+} \quad * \beta_{1}=\frac{\left[\mathrm{M}(\mathrm{OH})^{(\mathrm{z}-1)}\right]\left[\mathrm{H}^{+}\right]}{\left[\mathrm{M}^{\mathrm{z+}}\right]}=10^{-4.2} \\
& 2 \mathrm{M}^{z+}+2 \mathrm{H}_{2} \mathrm{O} \leftrightarrow \mathrm{M}_{2}(\mathrm{OH})_{2}^{(2 \mathrm{z}-2)}+2 \mathrm{H}^{+} \quad * \beta_{2,2}=\frac{\left[\mathrm{M}_{2}(\mathrm{OH})_{2}^{(2 \mathrm{z}-2)}\right]\left[\mathrm{H}^{+}\right]^{2}}{\left[\mathrm{M}^{z+}\right]^{2}}=10^{-7.7} \\
& 3 \mathrm{M}^{\mathrm{z+}}+4 \mathrm{H}_{2} \mathrm{O} \leftrightarrow \mathrm{M}_{3}(\mathrm{OH})_{4}^{(3 \mathrm{z}-4)}+4 \mathrm{H}^{+} \quad * \beta_{3,4}=\frac{\left[\mathrm{M}_{3}(\mathrm{OH})_{4}^{(3 \mathrm{z}-4)}\right]\left[\mathrm{H}^{+}\right]^{4}}{\left[\mathrm{M}^{\mathrm{z+}}\right]^{3}}=10^{-13.9}
\end{aligned}
$$

siguiendo un procedimiento similar al antes descrito se pueden obtener las expresiones para cada una de las fracciones molares del componente $\mathrm{M}$ en cada una de las especies químicas en solución: 


$$
\begin{aligned}
& \mathrm{F}_{\mathrm{M}}=\frac{\left[\mathrm{M}^{\mathrm{z}+}\right]}{\left[\mathrm{M}^{\mathrm{z}+}\right]+\left[\mathrm{M}(\mathrm{OH})^{\mathrm{z}-1}\right]+2\left[\mathrm{M}_{2}(\mathrm{OH})_{2}^{2 \mathrm{z}-2}\right]+3\left[\mathrm{M}_{3}(\mathrm{OH})_{4}^{3 \mathrm{z}-4}\right]} \\
& \mathrm{F}_{\mathrm{M}(\mathrm{OH})^{z-1}}=\frac{\left[\mathrm{M}(\mathrm{OH})^{\mathrm{z}-1}\right]}{\left[\mathrm{M}^{\mathrm{z}+}\right]+\left[\mathrm{M}(\mathrm{OH})^{\mathrm{z}-1}\right]+2\left[\mathrm{M}_{2}(\mathrm{OH})_{2}^{2 \mathrm{z}-2}\right]+3\left[\mathrm{M}_{3}(\mathrm{OH})_{4}^{3 \mathrm{z}-4}\right]} \\
& \mathrm{F}_{\mathrm{M}_{2}(\mathrm{OH})_{2}^{2 \mathrm{z}-2}}=\frac{2\left[\mathrm{M}_{2}(\mathrm{OH})_{2}^{2 \mathrm{z}-2}\right]}{\left[\mathrm{M}^{\mathrm{z}+}\right]+\left[\mathrm{M}(\mathrm{OH})^{\mathrm{z}-1}\right]+2\left[\mathrm{M}_{2}(\mathrm{OH})_{2}^{2 \mathrm{z}-2}\right]+3\left[\mathrm{M}_{3}(\mathrm{OH})_{4}^{3 \mathrm{z}-4}\right]} \\
& \mathrm{F}_{\mathrm{M}_{3}(\mathrm{OH})_{4}^{3 \mathrm{z}-4}}=\frac{3\left[\mathrm{M}_{3}(\mathrm{OH})_{4}^{3 \mathrm{z}-4}\right]}{\left[\mathrm{M}^{\mathrm{z}+}\right]+\left[\mathrm{M}(\mathrm{OH})^{\mathrm{z}-1}\right]+2\left[\mathrm{M}_{2}(\mathrm{OH})_{2}^{2 \mathrm{z}-2}\right]+3\left[\mathrm{M}_{3}(\mathrm{OH})_{4}^{3 \mathrm{z}-4}\right]} \\
& \mathrm{F}_{\mathrm{M}^{\mathrm{z+}}}=\frac{1}{1+* \beta_{1} /\left[\mathrm{H}^{+}\right]+2 * \beta_{2,2}[\mathrm{M}] /\left[\mathrm{H}^{+}\right]^{2}+3 * \beta_{3,4}[\mathrm{M}]^{2} /\left[\mathrm{H}^{+}\right]^{4}} \\
& \mathrm{~F}_{\mathrm{M}(\mathrm{OH})}^{\mathrm{z}-1}=\frac{* \beta_{1} /\left[\mathrm{H}^{+}\right]}{1+* \beta_{1} /\left[\mathrm{H}^{+}\right]+2 * \beta_{2,2}[\mathrm{M}] /\left[\mathrm{H}^{+}\right]^{2}+3 * \beta_{3,4}[\mathrm{M}]^{2} /\left[\mathrm{H}^{+}\right]^{4}} \\
& \mathrm{~F}_{\mathrm{M}_{2}(\mathrm{OH})_{2}^{2 \mathrm{z}-2}}=\frac{2 * \beta_{2,2}[\mathrm{M}] /\left[\mathrm{H}^{+}\right]^{2}}{1+* \beta_{1} /\left[\mathrm{H}^{+}\right]+2 * \beta_{2,2}[\mathrm{M}] /\left[\mathrm{H}^{+}\right]^{2}+3 * \beta_{3,4}[\mathrm{M}]^{2} /\left[\mathrm{H}^{+}\right]^{4}} \\
& \mathrm{~F}_{\mathrm{M}_{2}(\mathrm{OH})_{2}^{2 \mathrm{z}-2}}=\frac{3 * \beta_{3,4}[\mathrm{M}]^{2} /\left[\mathrm{H}^{+}\right]^{4}}{1+* \beta_{1} /\left[\mathrm{H}^{+}\right]+2 * \beta_{2,2}[\mathrm{M}] /\left[\mathrm{H}^{+}\right]^{2}+3 * \beta_{3,4}[\mathrm{M}]^{2} /\left[\mathrm{H}^{+}\right]^{4}}
\end{aligned}
$$

en estas expresiones es factible apreciar que cuando hay la presencia de especies polinucleares, las funciones se hacen dependientes de la concentración del componente sobre el cual se realiza el análisis, esto puede apreciarse también en los diagramas de distribución que se muestran en la figura A.1.2. Así se puede observar como a una concentración de $0.001 \mathrm{M}$ la fracción del mononuclear es mucho mayor que la del dimero, sin embargo cuando la concentración total del ion metálico es de $0.1 \mathrm{M}$ (figura A.1.2b) este comportamiento se invierte, además de que la región de $\mathrm{pH}$ en la que el trimero aparece se incrementa 


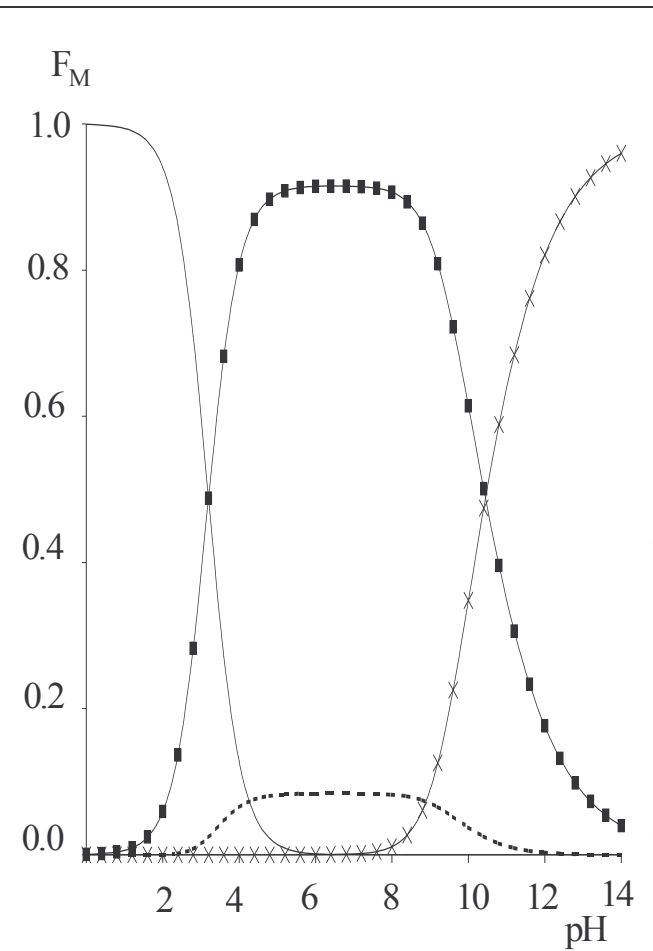

(a)

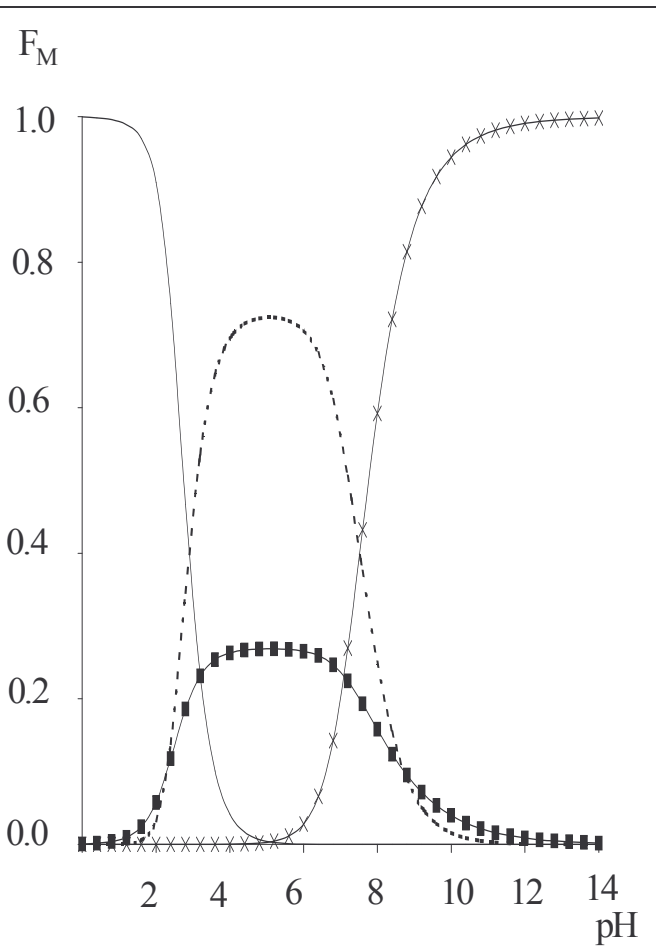

(b)

Figura A.1.2. . Diagramas de distribución de fracciones de componente para un sistema en el que

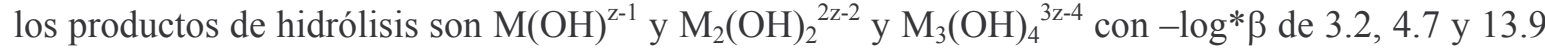
respectivamente ( - ) fracción molar de $\mathrm{M}^{\mathrm{z}+}$, (-+-) fracción molar de $\mathrm{M}(\mathrm{OH})^{\mathrm{z}-1}$, ( - - ) $\mathrm{M}_{2}(\mathrm{OH})_{2}{ }^{2 \mathrm{z}-2}$ (-X-) fracción molar de $\mathrm{M}_{3}(\mathrm{OH})_{4}{ }^{3 \mathrm{z}-4}$ (a) $\left[\mathrm{M}^{\mathrm{z}+}\right]_{\text {total }}=0.001 \mathrm{M}$; (b) $\left[\mathrm{M}^{\mathrm{z}+}\right]_{\text {total }}=0.10 \mathrm{M}$

\section{A.1.2.2. Fracciones Molares de Especie ${ }^{68}$}

Es posible plantear otro tipo de fracciones para las especies presentes en un sistema, en ellas se puede determinar la concentración molar de una especie en la solución entre la suma de las concentraciones de todas las especies presentes, estas fracciones nos permiten establecer la abundancia relativa de las especies en el sistema. Estas se definen por la ecuación A.1.10. 


$$
\mathrm{f}_{\mathrm{ij}}=\mathrm{f}_{\mathrm{M}_{\mathrm{i}}(\mathrm{OH})_{j}^{3 i-j}}=\frac{\left[\mathrm{M}_{\mathrm{i}}(\mathrm{OH})_{j}^{3 i-j}\right]}{\Sigma_{M}}=\frac{\left[\mathrm{M}_{\mathrm{i}}(\mathrm{OH})_{j}^{3 i-j}\right]}{\sum_{i}\left(\sum_{j}\left[\mathrm{M}_{\mathrm{i}}(\mathrm{OH})_{j}^{3 i-j}\right]\right)}
$$

La comparación de las ecuaciones A.1.3 y A.1.10 muestra claramente que las fracciones de componente y de especie son iguales cuando se tienen solamente especies mononucleares, sin embargo son diferentes si se presentan especies polinucleares en el sistema. Esto se debe a que en la definición de las fracciones del componente se considera el coeficiente estequiométrico del componente $\mathrm{M}$ como multiplicando en el numerador, $\mathrm{y}$ porque en ese caso la concentración total del componente $\left([\mathrm{M}]_{\text {total }}\right)$ es mayor que la suma simple de las concentraciones de las especies $\left(\Sigma_{\mathrm{M}}\right)$.

Retomando el ejemplo analizado anteriormente con especies polinucleares tendríamos que en este caso, las fracciones molares de especie se definirían por las ecuaciones mostradas en A.1.11 y A.1.12.

$$
\begin{aligned}
& f_{M}=\frac{\left[M^{z+}\right]}{\left[M^{z+}\right]+\left[M(O H)^{z-1}\right]+\left[M_{2}(\mathrm{OH})_{2}^{2 z-2}\right]+\left[M_{3}(\mathrm{OH})_{4}^{3 z-4}\right]} \\
& f_{M(\mathrm{OH})^{z-1}}=\frac{\left[M(\mathrm{OH})^{z-1}\right]}{\left[M^{z+}\right]+\left[M(\mathrm{OH})^{z-1}\right]+\left[\mathrm{M}_{2}(\mathrm{OH})_{2}^{2 z-2}\right]+\left[\mathrm{M}_{3}(\mathrm{OH})_{4}^{3 z-4}\right]} \\
& f_{M_{2}(\mathrm{OH})_{2}^{2 z-2}}=\frac{\left[\mathrm{M}_{2}(\mathrm{OH})_{2}^{2 z-2}\right]}{\left[\mathrm{M}^{z+}\right]+\left[M(\mathrm{OH})^{z-1}\right]+\left[\mathrm{M}_{2}(\mathrm{OH})_{2}^{2 z-2}\right]+\left[\mathrm{M}_{3}(\mathrm{OH})_{4}^{3 z-4}\right]} \\
& f_{M_{3}(\mathrm{OH})_{4}^{3 z-4}}=\frac{\left[M_{3}(\mathrm{OH})_{4}^{3 z-4}\right]}{\left[M^{z+}\right]+\left[M(\mathrm{OH})^{z-1}\right]+\left[\mathrm{M}_{2}(\mathrm{OH})_{2}^{2 z-2}\right]+\left[\mathrm{M}_{3}(\mathrm{OH})_{4}^{3 z-4}\right]}
\end{aligned}
$$




$$
\begin{aligned}
& f_{M^{z+}}=\frac{1}{1+* \beta_{1} /\left[H^{+}\right]+* \beta_{2,2}[M] /\left[H^{+}\right]^{2}+* \beta_{3,4}[M]^{2} /\left[H^{+}\right]^{4}} \\
& f_{M(\mathrm{OH})^{z-1}}=\frac{* \beta_{1} /\left[H^{+}\right]}{1+* \beta_{1} /\left[H^{+}\right]+* \beta_{2,2}[M] /\left[H^{+}\right]^{2}+* \beta_{3,4}[M]^{2} /\left[H^{+}\right]^{4}} \\
& f_{M_{2}(\mathrm{OH})_{2}^{2 z-2}}=\frac{* \beta_{2,2}[M] /\left[H^{+}\right]^{2}}{1+* \beta_{1} /\left[H^{+}\right]+* \beta_{2,2}[M] /\left[H^{+}\right]^{2}+\beta_{3,4}[M]^{2} /\left[H^{+}\right]^{4}} \\
& f_{M_{2}(\mathrm{OH})_{2}^{2 z-2}}=\frac{* \beta_{3,4}[M]^{2} /\left[H^{+}\right]^{4}}{1+* \beta_{1} /\left[H^{+}\right]+* \beta_{2,2}[M] /\left[H^{+}\right]^{2}+* \beta_{3,4}[M]^{2} /\left[H^{+}\right]^{4}}
\end{aligned}
$$

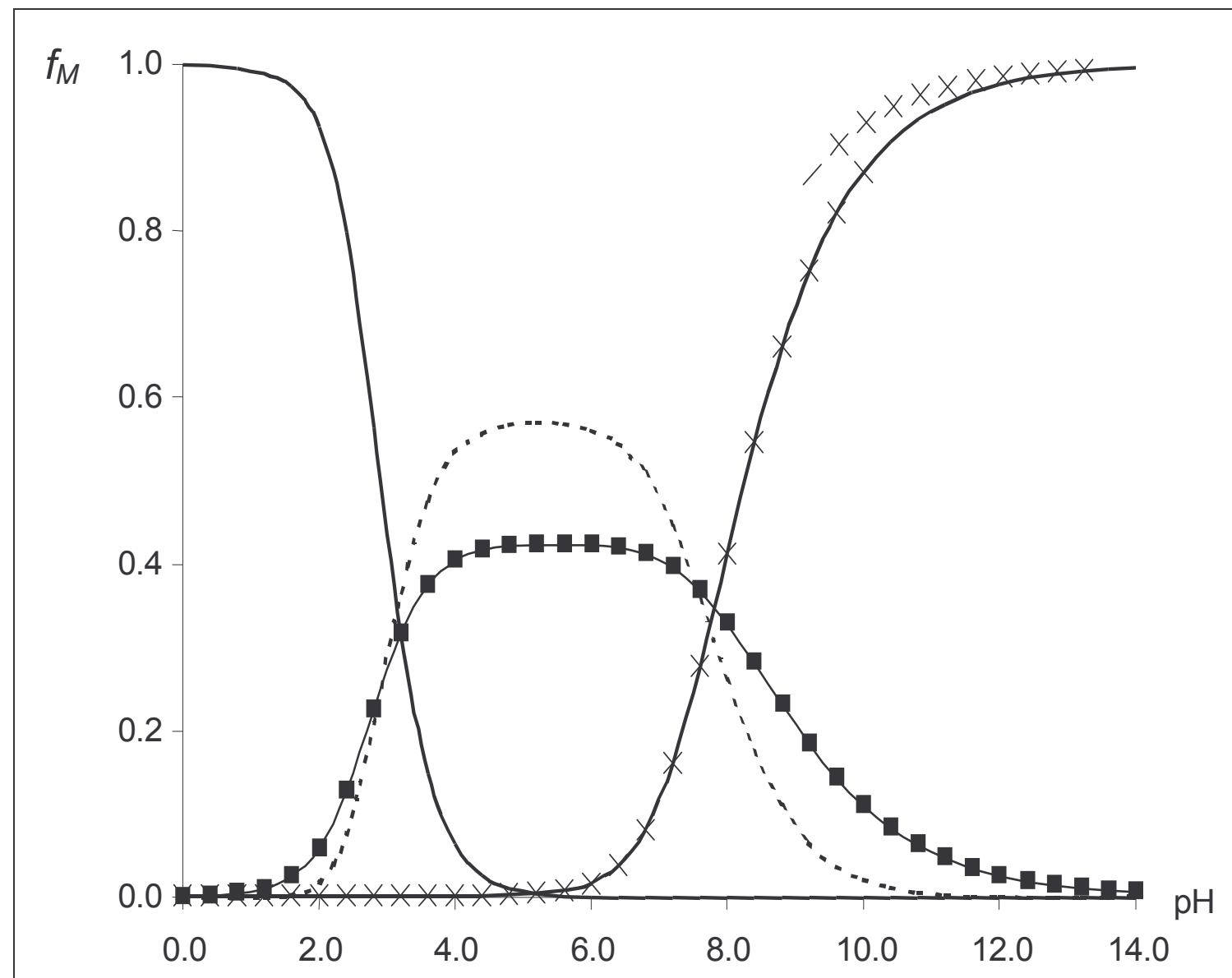

Figura A.1.3. . Diagramas de distribución de fracciones de especie para un sistema en el que los

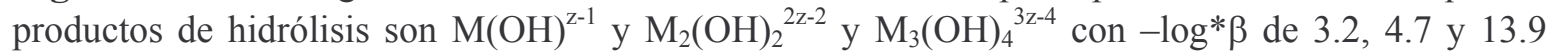
respectivamente, para $\left[\mathrm{M}^{\mathrm{z}+}\right]_{\text {total }}=0.10 \mathrm{M},(-)$ fracción molar de $\mathrm{M}^{\mathrm{Z}^{+}},(-+-)$fracción molar de $\mathrm{M}(\mathrm{OH})^{\mathrm{z}-1}$, ( - - ) $\mathrm{M}_{2}(\mathrm{OH})_{2}{ }^{2 \mathrm{z}-2}(-\mathrm{X}-)$ fracción molar de $\mathrm{M}_{3}(\mathrm{OH})_{4}{ }^{3 \mathrm{z}-4}$ 
La figura A.1.3 muestra el diagrama de distribución de fracciones molares de especies cuando la concentración del ion metálico es de $0.1 \mathrm{M}$, el cual se puede comparar con el diagrama de la figura A.1.2b y se puede apreciar que hay diferencias claras en las fracciones determinadas, así por ejemplo se puede observar que en el diagrama de fracciones de componente la fracción de ion metálico presente en el dimero es muy superior, prácticamente el doble, de la que se tiene en el monomero en un intervalo de $4.0 \leq \mathrm{pH} \leq 8.0$, sin embargo al comparar la diferencia entre las fracciones que se tienen en las concentraciones (fracciones de especie) esta no es tan marcada.

\section{A.1.3. NÚMERO PROMEDIO DE LIGANTES ${ }^{1,2,67,69}$}

Una función que ha sido de gran utilidad para poder conocer la proporción en la que el ion hidróxido se relaciona con el ion metálico es la función propuesta por Bjerrum

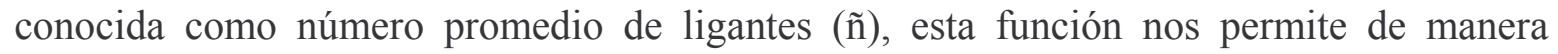
general conocer la relación molar de ligante (en nuestro caso $\mathrm{OH}^{-}$) asociados con el ion metálico. Esta función esta definida de manera general por la ecuación A.1.13, en la cual $\mathrm{C}_{\mathrm{L}}$ y $\mathrm{C}_{\mathrm{M}}$ representan las concentraciones totales de ligando y metal respectivamente y [L] representa la concentración libre de ligando. Si consideramos solamente los hidroxocomplejos mononucleares y las expresiones de ley de acción de masas de los complejos que se pudieran formar la función ñ puede representarse de acuerdo a A.1.14: 


$$
\begin{gathered}
\tilde{\mathrm{n}}=\frac{\mathrm{C}_{\mathrm{L}}-[\mathrm{L}]}{\mathrm{C}_{\mathrm{M}}} \\
\tilde{\mathrm{n}}=\frac{\left[\mathrm{MOH}^{z-1}\right]+2\left[\mathrm{M}(\mathrm{OH})_{2}^{z-2}\right]+\ldots+\mathrm{j}\left[\mathrm{M}(\mathrm{OH})_{j}^{z-j}\right]}{\left[\mathrm{M}^{z+}\right]+\left[\mathrm{MOH}^{z-1}\right]+\left[\mathrm{M}(\mathrm{OH})_{2}^{z-2}\right]+\ldots+\left[\mathrm{M}(\mathrm{OH})_{j}^{z-j}\right]} \\
\tilde{\mathrm{n}}=\frac{\beta_{1} /\left[\mathrm{H}^{+}\right]+2 \beta_{2} /\left[\mathrm{H}^{+}\right]^{2}+\ldots+j \beta_{\mathrm{J}} /\left[\mathrm{H}^{+}\right]^{J}}{1+\beta_{1} /\left[\mathrm{H}^{+}\right]+\beta_{2} /\left[\mathrm{H}^{+}\right]^{2}+\ldots+\beta_{\mathrm{J}} /\left[\mathrm{H}^{+}\right]^{J}}
\end{gathered}
$$

Si analizamos las expresiones anteriores, y sustituimos el conjunto de ecuaciones A.6 podemos obtener una expresión en función de las fracciones molares de componente (ecuación A.1.15)

$$
\tilde{\mathrm{n}}=\sum_{\mathrm{j}} \mathrm{j} \mathrm{F}_{\mathrm{j}}
$$

Es importante puntualizar que cuando solamente se tienen presentes especies mononucleares la función ñ no depende de la concentración total del ion metálico, y solamente depende de la concentración del ligando. La figura A.1.4 muestra el comportamiento del número promedio de ligantes en función del pH para sistemas en los que se supuso la formación de especies mononucleares únicamente, en ellos se puede apreciar que el valor máximo de la función se relaciona con el número máximo de ligantes que se pueden enlazar al ion metálico. 


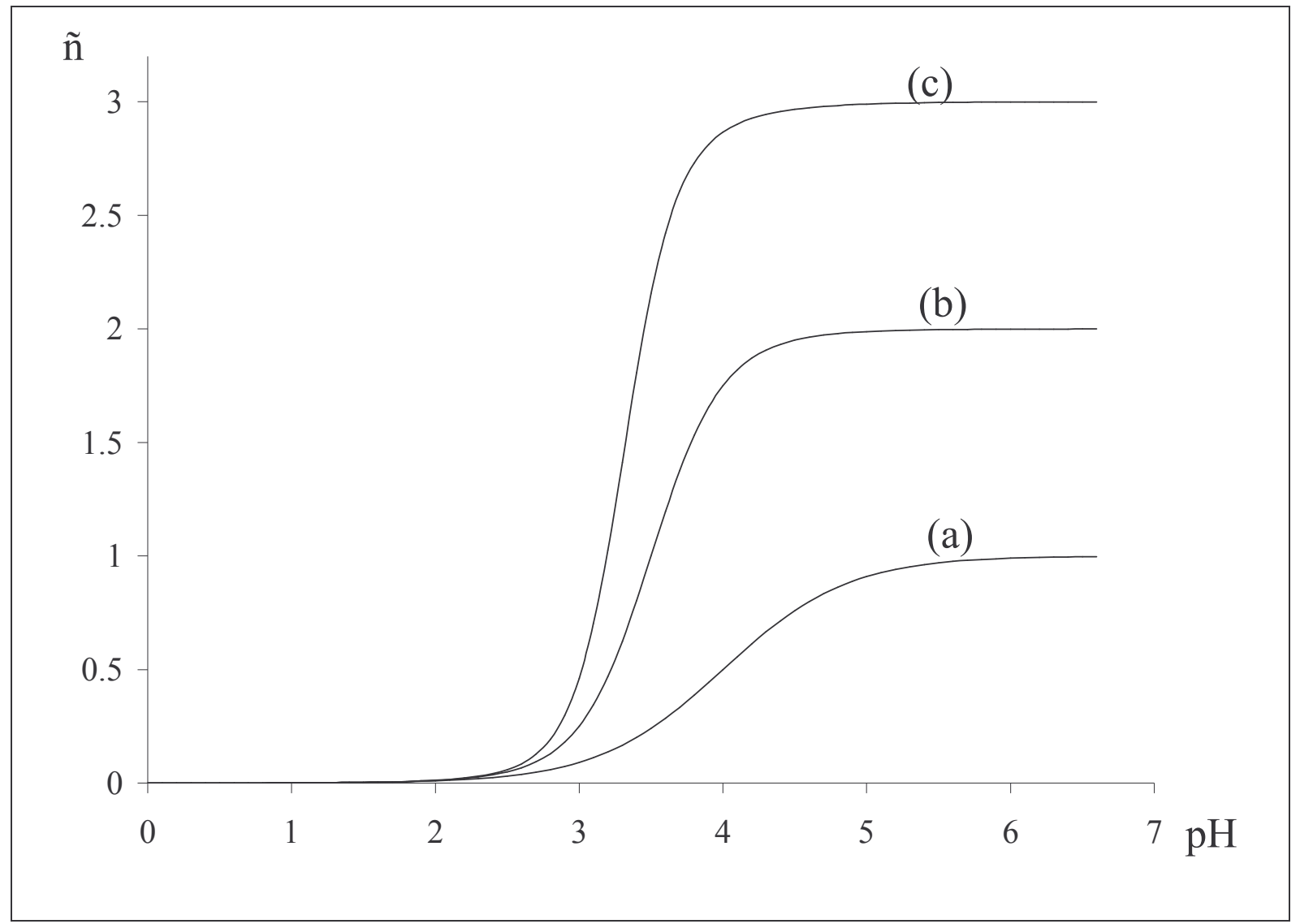

Figura A.1.4. Número promedio de ligandos (ñ), en función del $\mathrm{pH}$ para sistemas en los que se forman solamente especies mononucleares (a) $\mathrm{M}(\mathrm{OH})^{\mathrm{z}-1}$; (b) $\mathrm{M}(\mathrm{OH})^{\mathrm{z}-1}$ y $\mathrm{M}(\mathrm{OH})_{2}{ }^{\mathrm{z}-2}$, (c) $\mathrm{M}(\mathrm{OH})^{\mathrm{z}-1}$, $\mathrm{M}(\mathrm{OH})_{2}{ }^{\mathrm{z}-2}$ y $\mathrm{M}(\mathrm{OH})_{3}{ }^{\mathrm{z}-3}\left[\mathrm{M}(\mathrm{OH})^{\mathrm{z}-1} \quad-\mathrm{Log} * \beta_{1}=4.0, \quad \mathrm{M}(\mathrm{OH})_{2}{ }^{\mathrm{z}-2} \quad-\mathrm{Log} * \beta_{2}=7.0\right.$ y $\mathrm{M}(\mathrm{OH})_{3}{ }^{\mathrm{z}-3}$ $\left.\log * \beta_{3}=10.0\right]$

Cuando en el sistema se tiene especies polinucleares se puede plantear una función similar a la del número promedio de ligantes que si bien operacionalmente tiene la misma definición, el significado de ésta de acuerdo con Sillen $^{69}$ cambia, ya que no es posible apreciar la cantidad de ligandos unidos al ion metálico central, sino que ahora la función se vuelve dependiente de la concentración tanto de ligante como de metal, a ésta se le da el nombre de función de formación $(Z)$. Dicha función suele emplearse para poder decidir si 
los complejos presentes en un sistema son mononucleares o polinucleares, si en el sistema se forman estos últimos, una variación en la concentración del metal generará un desplazamiento en la curva, de forma tal que entre mayor sea la concentración, mayor será la fracción de complejos polinucleares y lo contrario ocurrirá al diluirse la solución. Al llegar a cierta dilución se forma lo que se conoce como "pared mononuclear" lo que implica que si la concentración del metal sigue disminuyendo la curva permanecerá constante, ya que se por la dilución se habrá alcanzado el estado mononuclear en el sistema.

La ecuación que describe a esta función considera ahora a la cantidad de núcleos metálicos enlazados a cada ligante en las especies polinucleares y se describe en la ecuación A.1.16.

$$
Z=\sum_{i}\left(\sum_{j}\left(\frac{j}{i}\right) F_{i j}\right)
$$

En la figura A.1.5 se ilustra el comportamiento de la función $Z$ para el sistema propuesto en el apartado de A.1.2.2 en el que se supuso una especie mononuclear $(1,1)$, un dimero $(2,2)$ y un trímero $(3,4)$, en ella se puede apreciar que conforme se modifica la concentración total del metal la gráfica sufre desplazamiento, además se observa que al tener concentraciones menores de $10^{-6} \mathrm{M}$ se presenta la "pared mononuclear", ya que la función presenta el mismo comportamiento. 


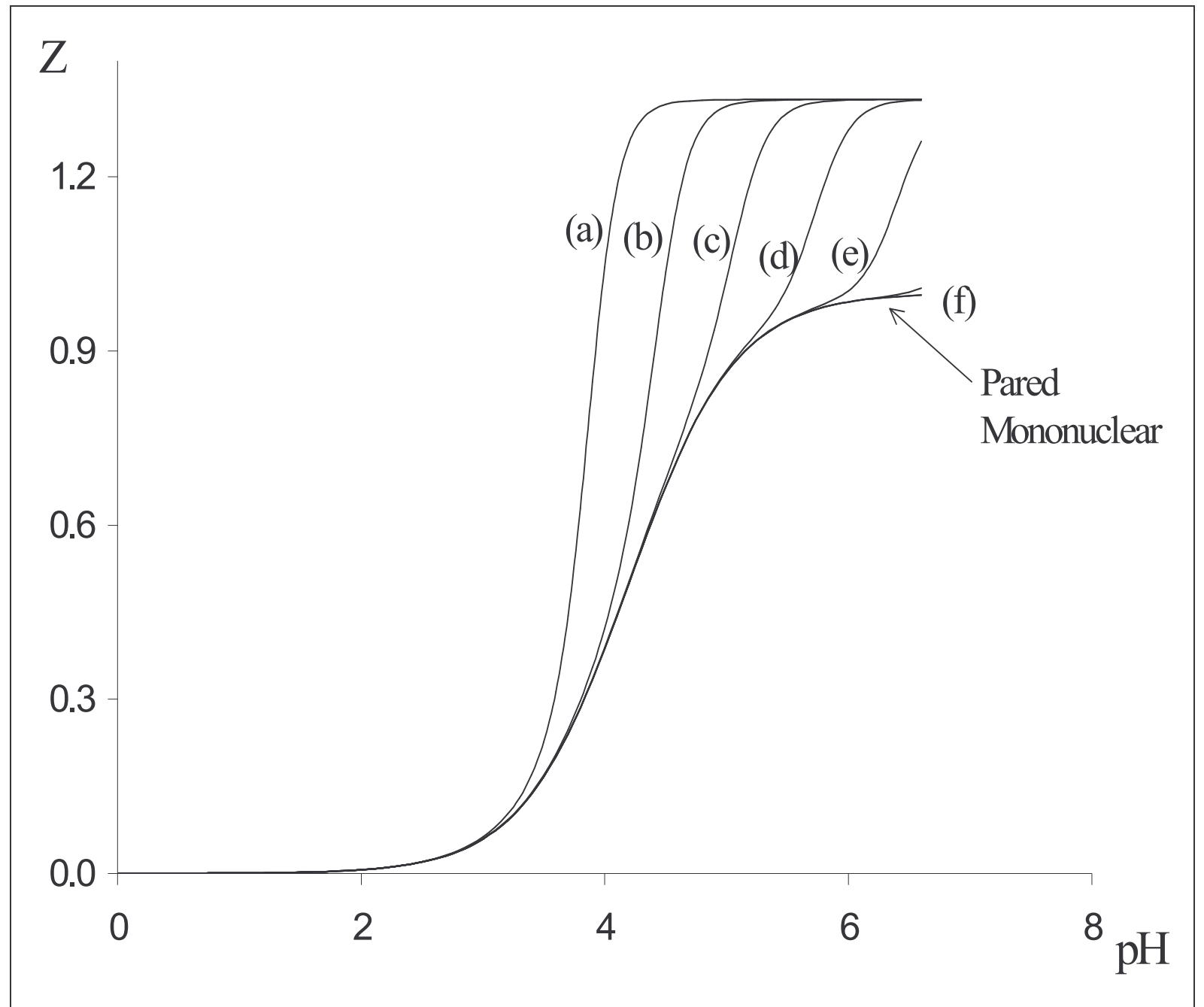

Figura A.1.5. Función de formación $(\mathrm{Z})$ para un sistema en el que se forman $\mathrm{M}(\mathrm{OH})^{\mathrm{z}-1} \mathrm{y}$ $\mathrm{M}_{2}(\mathrm{OH})_{2}{ }^{2 \mathrm{z}-2}$ y $\mathrm{M}_{3}(\mathrm{OH})_{4}{ }^{3 \mathrm{z}-4}$ con $-\log { }^{*} \beta$ de $3.2,4.7$ y 13.9 respectivamente, (a) $\left[\mathrm{M}^{\mathrm{z}+}\right]_{\text {total }}=10^{-1} \mathrm{M}$; (b) $\left[\mathrm{M}^{\mathrm{Z}+}\right]_{\text {total }}=10^{-2} \mathrm{M}$; (c) $\left[\mathrm{M}^{\mathrm{z}+}\right]_{\text {total }}=10^{-3} \mathrm{M}$; (d) $\left[\mathrm{M}^{\mathrm{z}+}\right]_{\text {total }}=10^{-4} \mathrm{M}$; (e) $\left[\mathrm{M}^{\mathrm{z}+}\right]_{\text {total }}=10^{-5} \mathrm{M}$; (f) $\left[\mathrm{M}^{\mathrm{z}+}\right]_{\text {total }} \leq 10^{-6}$ $\mathrm{M}$

Cuando se tiene información experimental y se desea obtener la función de formación, para poder hacer la comparación con aquella obtenida a partir de un modelo químico, es necesario conocer los valores de $\mathrm{pH}$ (por consecuencia la concentración de $\mathrm{H}^{+}$) y la concentración total del ion metálico. La expresión que permite realizar este calculo se 
expresa en la ecuación A.1.17

$$
\mathrm{Z}=\frac{\left[\mathrm{OH}^{-}\right]_{\text {total }}-\left[\mathrm{OH}^{-}\right]+\left[\mathrm{H}^{+}\right]}{\left[\mathrm{M}^{\mathrm{z}+}\right]_{\text {total }}}
$$

en donde $\left[\mathrm{OH}^{-}\right]_{\text {total }}$ es igual a la cantidad total de iones hidróxido adicionados al sistema, $\left[\mathrm{OH}^{-}\right]$es la concentración del ion hidróxido proveniente del agua y $\left[\mathrm{H}^{+}\right]$es la concentración de protones adicionados, por lo que la ecuación se puede representar como:

$$
\mathrm{Z}=\frac{\left[\mathrm{OH}^{-}\right]_{\text {total }}-\left[\mathrm{K}_{\mathrm{w}} / 10^{-\mathrm{pH}}\right]+\left[10^{-\mathrm{pH}}\right]}{\left[\mathrm{M}^{\mathrm{z}+}\right]_{\text {total }}}
$$

La obtención de esta función a partir de datos experimentales permite el poder hacer una comparación entre esta y la obtenida con base en un modelo propuesto y por tanto resulta una herramienta importante para poder establecer que tan adecuado puede resultar el modelo propuesto. 


\section{APÉNDICE 2 \\ CONCEPTOS BÁSICOS DE RESONANCIA MAGNÉTICA NUCLEAR (RMN)}

\section{A.2.1. ANTECEDENTES}

La proposición de que los núcleos atómicos poseen momentos magnéticos se debe a Linus Pauling en 1920. En 1946 Purcell y Bloch reportaron independientemente que la resonancia magnética nuclear del protón podía observarse en la parafina y el agua, esto les valió el premio Nóbel de química en 1952. A partir de los años cincuentas se comenzaron a utilizar los primeros equipos de alta resolución, de tan sólo $40 \mathrm{MHz}$, empezándose con estos instrumentos el esplendor de la química orgánica. Por la misma época se comenzaron a observar los núcleos inorgánicos más fáciles como el ${ }^{19} \mathrm{~F},{ }^{10} \mathrm{~B},{ }^{11} \mathrm{~B}$ y ${ }^{31} \mathrm{P}$ sin embargo eran necesarias grandes cantidades de muestra lo cual restringía su determinación ${ }^{70}$.

A principios de 1970 con el advenimiento de las técnicas de pulso con transformada de Fourier, que permite obtener espectros en tiempos muy cortos y además acumularlos, se mejoró la sensibilidad y se comenzaron a estudiar isótopos que se encontraban en baja concentración o que eran difíciles de observar. A partir de ese momento con esta técnica empieza una nueva etapa para la RMN. Actualmente con los superconductores se obtienen campos magnéticos mas potentes con lo cual se mejora la sensibilidad ${ }^{70}$.

\section{A.2.2. PROPIEDADES NUCLEARES Y LA CONDICIÓN DE RESONANCIA.}


Los núcleos que poseen un momento angular de espín $\boldsymbol{I}$ tienen asociado un campo magnético y se comportan como dipolos magnéticos, el cual tiende a alinearse con un campo magnético aplicado y a interactuar con dipolos vecinos. El momento magnético $\boldsymbol{\mu}$ que determina la energía potencial $E$ del dipolo magnético nuclear en un campo magnético de fuerza $B o$ esta definido por la ecuación A.2.1 $1^{70,71}$.

$$
E=-\boldsymbol{\mu} B o
$$

En presencia de un campo magnético los núcleos se alinean en varias orientaciones permitidas, dadas por los componentes observables de $\boldsymbol{I} M_{i} \mathbf{h}$, siendo $M_{I}$ el número cuántico magnético $\left(M_{I}\right)$ que puede tomar los valores de $\boldsymbol{I},(\boldsymbol{I}-1), \ldots .,(-\boldsymbol{I}+1),-\boldsymbol{I}$, que corresponden a los diferentes niveles de energía Zeeman. Generalmente el magnetismo de un núcleo se describe en términos de su relación magnetogírica $(\gamma)$, que es una constante de proporcionalidad que describe el magnetismo de cada núcleo y relaciona el momento magnético angular con el momento angular de espín (ecuación A.2.2) 71,72. $^{7}$.

$$
\gamma=\boldsymbol{\mu} / \boldsymbol{I}
$$

Si consideramos las ecuaciones A.2.1 y A.2.2, así como la cuantización de $\boldsymbol{I}$ en sus componentes observables $M_{l} \mathbf{h}$ en la dirección de un campo magnético Bo se obtiene: 


$$
E=-\gamma \mathbf{h} M_{I} B o
$$

En la RMN se inducen transiciones del espín nuclear entre los niveles de energía de $M_{I}$ mediante el empleo de radiación electromagnética del orden de 50-500MHz, debido a que la regla de selección es $\Delta M_{I}= \pm 1$ se tiene que para un núcleo la diferencia de energía $(\Delta E)$ es la misma entre las diferentes transiciones obteniéndose la ecuación A.2.4 que es la

$$
\Delta E=\gamma \mathbf{h} B o=\mathrm{h} v_{\mathrm{o}}
$$

condición de resonancia para que haya absorción de energía, en donde $v_{\mathrm{o}}$ se conoce como la frecuencia de $\operatorname{Larmor}^{70,71}$.

La absorción de radiación electromagnética puede ocurrir gracias a que existe una diferencia en la población de los niveles de energía más bajos con respecto a los altos en un equilibrio térmico. La ley de Boltzmann da la relación de poblaciones entre los estados de energía (ecuación A.2.5)

$$
\mathrm{n}_{2} / \mathrm{n}_{1}=\exp \left(\mathbf{h} v_{\mathrm{o}} / \mathrm{kT}\right)=\exp (\gamma \mathrm{h} B o / \mathrm{kT})
$$

Cuando las poblaciones se igualan no hay emisión espontánea de la radiación, el sistema se satura, la forma como se disipa la energía es a través de los campos magnéticos locales que fluctúan a la frecuencia de Larmor que inducen transferencia no radiactivas 
entre niveles de energía magnética nuclear, esto se conoce como el fenómeno de relajamiento $^{70,71}$.

Para evitar la baja sensibilidad, las señales de RMN se acumulan mediante una serie de experimentos repetidos, debido a que la relación señal-ruido se incrementa solamente como $(\mathrm{n})^{1 / 2}$ donde $\mathrm{n}$ es el número de experimentos, un factor $\chi$ en la receptividad corresponde a un factor $\chi^{2}$ en el tiempo de acumulación. de particular importancia es la velocidad de restablecimiento de la distribución de Boltzmann (equilibrio térmico) ya que la velocidad de repetición depende de ello ${ }^{70,71}$.

\section{A.2.3. PRINCIPALES PARÁMETROS DE RMN.}

Los principales parámetros de $\mathrm{RMN}$, la protección magnética nuclear, el acoplamiento espín-espín $(J)$ y el acoplamiento dipolar directo $(D)$, son cantidades tensoriales, ya que el ambiente en el cual el núcleo se encuentra no es generalmente simétricamente esférico. Todos ellos son tensores debido a que son propiedades originadas de la interacción del vector de espín nuclear $\boldsymbol{I}$ con algún otro vector, es decir con Bo (protección), con $\boldsymbol{I}$ vía un cuadrupolo eléctrico nuclear (acoplamiento cuadrupolar), o con otro espín nuclear I' (acoplamiento $D$ ó $J$ ). Si todos estos acoplamientos fueran evidentes en el espectro de RMN sería algo realmente complicado. Sin embargo, en mediciones en fases líquido o gas estos e simplifica enormemente debido al movimiento desordenado de la 
molécula, con rápidas rotaciones interrumpidas por frecuentes colisiones, lo cual lleva a la muestra a todas las posibles orientaciones con igual probabilidad ${ }^{71}$.

\section{A.2.3.1. Desplazamiento Químico y el Tensor de Protección.}

Gran parte de la información en los espectros de RMN surge del desplazamiento químico debido a los campos magnéticos locales generados en el núcleo por la circulación de los electrones circundantes inducidos por el campo aplicado. Si los campos magnéticos resultantes se oponen al campo aplicado $(B o)$ el efecto es la protección o apantallamiento del núcleo. El núcleo entonces experimenta un campo efectivo descrito por la ecuación A.2.6. en la cual $\sigma$ es la protección magnética nuclear y se expresa en unidades de $10^{-6}$, o

$$
B_{\text {efec }}=B o(1-\sigma)
$$

partes por millón (ppm). Los elementos del tensor $\sigma$ reflejan la simetría del ambiente electrónico del núcleo, dependiendo del tipo de enlace, sin embargo esta no es una referencia práctica. Es más usual establecer el desplazamiento químico $(\delta)$ el cual es comúnmente medido en relación a una sustancia estándar, estando definido por:

$$
\delta / \mathrm{ppm} \equiv 10^{6}\left(\nu_{\mathrm{m}}-v_{\mathrm{ref}}\right) / \nu_{\text {ref }}
$$


el cual puede ser relacionado con la diferencia de protección en el límite $\sigma<<1$, de manera que $\delta \approx \sigma_{\text {ref }}-v_{\mathrm{m}}$, en donde el subíndice $m$ se refiere a la muestra y ref a la referencia ${ }^{71}$.

\section{A.2.3.2. Acoplamiento Dipolar (D).}

La presencia de núcleos magnéticos vecinos altera el campo local y por ende la energía del núcleo. La interacción dipolar directa entre dos momentos magnéticos $\boldsymbol{\mu}_{\mathbf{1}} \mathrm{y} \boldsymbol{\mu}_{\mathbf{2}}$ separados por un vector $r$ es:

$$
E_{\mathrm{D}}=\left(\mu_{1} \cdot \mu_{2}\right) / r^{3}-3\left(\mu_{1} \cdot r\right)\left(\mu_{2} \cdot r\right) / r^{5}
$$

y para dipolos nuclear magnéticos se tiene:

$$
E_{D}=\gamma_{1} \gamma_{2} \mathbf{h}^{2}\left[\left(\mathbf{I}_{1} \cdot \mathbf{I}_{2}\right) / r^{3}-3\left(\mathbf{I}_{1} \cdot r\right)\left(\mathbf{I}_{2} \cdot r\right) / r^{5}\right]
$$

que puede ser escrita en forma abreviada como:

$$
E_{D}=\gamma_{1} \gamma_{2} \mathbf{h}^{2} \mathbf{I}_{1} D \mathbf{I}_{2}
$$

en donde $D$ es el tensor de acoplamiento dipolar directo. Se puede observar que $D$ solamente depende de las coordenadas relativas de posición del núcleo 1 y 2. 
El efecto de la interacción dipolar en los espectros de RMN depende del ángulo $\theta$ que formen el vector internuclear con el campo magnético $B o$. De la interacción $\mathbf{I}_{\mathbf{1}} \cdot D \mathbf{I}_{\mathbf{2}}$ resultan seis clases diferentes de términos, de los cuales solamente dos contribuyen en primer orden, los cuales tienen el factor $\left(3 \cos ^{2} \theta-1\right) / r^{3}$. La dependencia de $r^{-3}$ significa que el acoplamiento dipolar es fuerte en distancias cortas y disminuye rápidamente con la distancia $^{71}$.

En fase líquida el promedio isotrópico de $\left(3 \cos ^{2} \theta-1\right)$ sobre todas las orientaciones es cero, así que las interacciones dipolares no contribuyen a la posición en los espectros de RMN en fase líquida. en sólidos o en líquidos cristalinos en los cuales las posiciones nucleares se mantienen relativamente al campo aplicado, la interacción directa de los dipolos magnéticos puede ser observada en los espectros de $\mathrm{RMN}^{71}$.

\section{A.2.3.3. Acoplamiento Indirecto Espín-Espín (J).}

Un acoplamiento entre dos núcleos cuyo promedio no es cero en líquidos es el acoplamiento indirecto espín-espín o acoplamiento $J$; en efecto la interacción total (dipolar)

entre momentos nucleares magnéticos es $\gamma_{1} \gamma_{2} \mathbf{h}^{2} \mathbf{I}_{1}(J+D) \mathbf{I}_{2}$. Para las moléculas orientadas los componentes de $(J+D)$ son evidentes en el espectro. En fases isotrópicas solamente $J$ es observado, debido a que la interacción es mediante los electrones en los enlaces entre los núcleos. Básicamente existen tres mecanismos principales para el acoplamiento indirecto $J$, todos ellos mediados por los electrones de valencia. El más importante de ellos $\left(J_{A B}{ }^{F c}\right)$ involucra la interacción (contacto de Fermi) de un electrón $s$ con el núcleo (con el cual los 
electrones $\mathrm{p}, \mathrm{d}, \ldots$ no tienen contacto). Otro mecanismo de acoplamiento espín-espín involucra la interacción mediada por los electrones $\mathrm{p}, \mathrm{d}, \ldots$. El termino orbital $J_{A B}{ }^{o}$ surge de las perturbaciones de los campos magnéticos debidas al movimiento orbital de los electrones por el núcleo $\mathrm{A}$, esta perturbación es experimentada por el núcleo $\mathrm{B}, \mathrm{y}$ viceversa. El término espín-dipolar $J_{A B}^{\text {sd }}$ surge de la directa interacción del dipolo magnético del núcleo $\mathrm{A}$, con el dipolo magnético del electrón orbital, el cual interactúa con el núcleo B. La constante de acoplamiento observada es la suma de $f^{f c}$ (el cual generalmente domina) y de $J^{o}$ y $J^{s d}$. Estos componentes solamente pueden ser evaluados por cálculos teóricos y muestran la importancia de los electrones de no enlace, así como de los electrones de enlace $\sigma \mathrm{y} \pi^{71}$

\section{A.2.3.4. Cuadrupolo Eléctrico.}

Todos los núcleos con $\boldsymbol{I}>1 / 2$ tienen una distribución elipsoidal de la carga y un momento eléctrico cuadrupolar. La energía electrostática es minimizada por el apropiado alineamiento de un cuadrupolo en un gradiente de campo. En una molécula, hay un gradiente de campo eléctrico (gce) debido a la asimetría en la distribución de la carga local debida a los electrones y a otros núcleos. La energía de un cuadrupolo nuclear es cuantizada de acuerdo a su orientación en el gradiente de campo eléctrico siempre en ausencia de un campo magnético externo. Las transiciones son inducidas por una radiofrecuencia, en lo que es conocido como resonancia cuadrupolar nuclear, esto lleva generalmente a la obtención de bandas de absorción ensanchadas ${ }^{71,72}$. 


\section{A.2.3. DETERMINACIÓN DEL ÁREA PARA LAS SEÑALES DE RMN.}

Se han realizado muy pocos estudios de RMN enfocados hacia la determinación o análisis cuantitativo de especies químicas ${ }^{7,8}$, la mayoría de los estudios se han enfocado a la determinación del tipo de especies químicas que se tienen presentes o bien a la elucidación de las geometrías estructurales de dichas especies.

En este trabajo se propone una metodología que permita relacionar las señales de resonancia magnética nuclear $(\mathrm{RMN})$ con las fracciones molares de las especies formadas durante el proceso de hidrólisis, para ello es necesario determinar el área bajo el espectro de resonancia. Para este fin las señales obtenidas se integran, realizando esta integración con ayuda del software del instrumento. Lo anterior se efectúa tomando en consideración que las señales presentan un comportamiento gaussiano o pseudogaussiano. Por otro lado, cuando se tienen señales complejas que se alejan del comportamiento antes mencionado se procede a la deconvolución con base en que dicha señal esta integrada por diversas señales espectrales, todas ellas con características gaussianas. 